\title{
Nav1.5 Modulation: From Ionic Channels to Cardiac Conduction and Substrate Heterogeneity
}

\author{
Dissertation \\ for the award of the degree \\ "Doctor rerum naturalium" \\ Division of Mathematics and Natural Sciences \\ of the Georg-August-Universität Göttingen
}

Submitted by

Nour RAAD

from Beirut, Lebanon

Göttingen, December 12, 2013 


\section{Members of Thesis Committee:}

1. Prof. Dr. Stefan Luther (First Reviewer)

2. Prof. Dr. Stephan E. Lehnart

3. Prof. Dr. Eberhard Bodenschatz

\section{Members of the Examination Board:}

1. Prof. Dr. Dörthe Katschinski (Second Reviewer)

2. Prof. Dr. Ulrich Parlitz

3. Dr. Viacheslav Nikolaev

Date of Oral Examination: January 16 ${ }^{\text {th }}, 2014$ 
I hereby declare that this thesis has been written independently and with no other sources and aids than quoted.

Nour Raad 
To my parents and sisters...

"I found the task so truly arduous... that I was almost tempted to think... that the movement of the heart was only to be comprehended by God."

Sir William Harvey,

De motu Cordis, 1628 


\section{Acknowledgments}

My academic education started in Beirut in 2002, when I first enrolled as a Physics pre-medical student at the American University of Beirut. Although choosing Physics as a major is not common, since both curricula are demanding and competitive, I believed then that this discipline would offer me the potential to understand the dynamic nature of complex systems, from their fundamental and evolutionary design to their function and performance. This knowledge allowed me to experience medical school from a special perspective: I came to learn that the sophistication of our physiology commences with a sequence of entangled molecular events that result in even more convoluted biophysical processes, which only by working cooperatively and constructively give birth to human intelligence, creativity and passion. I also learned that my help as a physician would be needed when any of these processes end up failing. Alleviating pain and treating diseases are perhaps humankind's greatest achievements, but the harder and more demanding task lies beneath these achievements, through decades of endless scientific search for why these highly organized processes have failed in the first place.

Undertaking this $\mathrm{PhD}$ has undoubtedly been a life-changing experience for me. This intermingled multidisciplinary thesis in physics and cardiology was carried in the last four years as a balance between experimental and theoretical work, which offered me the pleasure of exploring the translational continuum of molecular events into their pathophysiological processes at the level of the whole heart. I certainly wouldn't have made it this far without the guidance, help and support of so many people, first of whom I would like to thank, would be my supervisor Prof. Stefan Luther.

There are hardly words to thank him enough or to explain how I came to admire and respect him, not just in his way of doing science, but also for the academic leader I found in him. He didn't only make a difference in my life as his PhD student, but also in the lives of whoever worked with him: students who have been blessed to have had him teach them, and colleagues who all hold him with such great esteem. Having no experience in optical mapping whatsoever when I first started, I found the confidence I needed to go every mile of my $\mathrm{PhD}$ in him. Four years later, I come to believe that so much of his patience, guidance and hopefully experience are now in me, which I will carry with me every day of my career in medicine and science.

The second person on my list would be my colleague and dear friend Philip Bittihn. I would probably remember the endless discussions over nonsensical data, Matlab scripts that don't work or unexpected daily problems in the lab. However, what I am certain of, is that I will never forget when hard times seemed to never end, he was there to remind me to keep faith and go on. I feel honored to have had him as an incredibly smart colleague and trusted friend.

Many thanks of course to my thesis committee members: Prof. Eberhard Bodenschatz and Prof. Stephan Lehnart, whom without their constant guidance and feedback, this PhD would not have 
been achievable. Prof. Lehnart additionally had offered me the chance to work both independently and cooperatively in his lab for a considerable time of my $\mathrm{PhD}$ and I am highly appreciative for his patience and guidance regarding the experimental demands and challenges of this work. In addition, I would like to thank all members of my examination board including Prof. Ulrich Parlitz, Dr. Viacheslav Nikolaev and particularly Prof. Dörthe Katschinski, whom I won't forget her precious advise, scientific feedback and unending encouragement to pursue a dual scientific and clinical career.

I would to also thank my colleagues and members of the Biomedical Physics Group in the Max Planck Institute for Dynamics and Self-Organization, particularly Johannes Schöder-Schetelig, Amgad Squires and Alexander Schlemmer for all the help they gave me in the beginning of my experimental and analytical work. I would like to also thank members of the Translational Cardiology Group in the University Hospital of Göttingen, especially Karen Presting, Eva Wagner, Brigitte Korff, Konstantin Gusev and Miroslav Dura for their support, feedback and suggestions.

I am also grateful to the International Max Planck Research School for Physics of Biological and Complex Systems, for funding the first three years of my PhD work and providing me with a great research environment that has been a crucial component for the progress of my doctoral work. In that regard, I would also like to thank Mrs. Antje Erdmann for her help during the application procedures and Mrs. Frauke Bergmann for her patience during the thesis submission and defense procedures.

Some people were not directly involved in my thesis work, however I know that I wouldn't be the person I am today without their presence in my life. First and foremost, I would like to thank Dr. Salah Chouairi for making me fall in love with cardiac electrophysiology almost from the first lecture in medical school. I have always seen in him a role model of the physician I would like to become. I can never thank my family enough for all they did for me to get me here: my father for his sacrifice, patience, and love; my mother for worrying about me every single day and night since the day I was born, for her precious input into my education and endless love; both my sisters Farah and Laura and my best friend Rana for all the times we spent together, for keeping their sense of humor when I had lost mine and for their selfless love and care that kept me going.

Sincerely,

Nour Raad 


\title{
List of Abbreviations
}

\author{
AADs \\ AF \\ Ao \\ Ap \\ AP \\ APD \\ $\mathrm{APD}_{\mathrm{xx}}$ \\ $\triangle \mathrm{APD}_{\mathrm{xx}}$ \\ AR \\ ATP \\ AVN \\ bpm \\ CaM \\ CaMKII \\ CASQ \\ CB \\ CICR \\ CPVT \\ CV \\ Cx43 \\ DAD \\ DHPR \\ DMD \\ DSMC \\ $(\mathrm{dV} / \mathrm{dt})_{\max }$ \\ $(d F / d t)_{\max }$ \\ EAD \\ ECC \\ ECG \\ ECM \\ FC \\ GJ \\ HF \\ $\mathbf{I}_{\mathrm{Na}} ; \mathbf{I}_{\mathbf{N a}, \mathbf{f}}$ \\ $\mathbf{I}_{\mathrm{Na}, \mathbf{p}}$ \\ $\mathbf{I}_{\mathbf{t o}, \mathbf{1}}$ \\ AntiArrhythmic Drugs \\ Area Fitting \\ Aorta \\ Apex \\ Action Potential \\ Action Potential Duration \\ Action Potential Duration with $\mathbf{x x} \%$ repolarization from the peak \\ Dispersion of repolarization of $\mathbf{A P D}_{\mathbf{x x}}$ \\ Anisotropic Ratio \\ Adenosine TriPhosphate \\ AtrioVentricular Node \\ beats per minute \\ CalModulin \\ Calcium/CalModulin-dependent protein Kinase $\mathbf{2}$ \\ CAlSeQuestrin \\ Conduction Block \\ Calcium Induced Calcium Release \\ Catecholaminergic Polymorphic Ventricular Tachycardia \\ Conduction Velocity \\ Connexin 43 \\ Delayed AfterDepolarization \\ DiHydroPyridine Receptor \\ Duchenne Muscular Dystrophy \\ Dystrophin Syntrophin Multiprotein Complex \\ Maximum upstroke velocity (as the actual membrane potential changes \\ per unit time) \\ Maximum upstroke velocity (as the optical voltage signal changes per unit \\ time) \\ Early AfterDepolarization \\ Excitation Contraction Coupling \\ ElectroCardioGram \\ ExtraCellular Matrix \\ Fractional Change (in \%) \\ Gap Junction \\ Heart Failure \\ Fast inward Na-current (I) \\ Persistent (or late) inward Na-current \\ Transient Outward K-current
}




\begin{tabular}{|c|c|}
\hline $\mathbf{I}_{\mathbf{C a}, \mathbf{L}}$ & Long-lasting Ca-current \\
\hline ICD & Implantable Cardioverter Defibrillator \\
\hline ID & Intercalated Disk in the cardiomyocyte \\
\hline JN & JunctiN \\
\hline LA & Left Atrium \\
\hline LCR & Local Calcium Release \\
\hline LM & Lateral Membrane of the cardiomyocyte \\
\hline LQTS3 & Long QT Syndrome 3 \\
\hline LSEF & Least Squares Ellipsis Fitting \\
\hline $\mathbf{L V}$ & Left Ventricle \\
\hline$m d x$ & muscular dystrophin deficient mouse model \\
\hline Nav1.5 & Voltage gated cardiac sodium (Na)-channel \\
\hline NCX & Na-Ca eXchanger \\
\hline OAP & Optical Action Potential \\
\hline PE & Pacing Electrode \\
\hline PF & Plane Fitting \\
\hline PKA & Protein Kinase A \\
\hline PLB & PhosphoLamBan \\
\hline PVC & Premature Ventricular Complex \\
\hline RA & Right Atrium \\
\hline ROI & Region Of Interest \\
\hline $\mathbf{R P}$ & Refractory Period \\
\hline RV & Right Ventricle \\
\hline RyR & Ryanodine Receptor \\
\hline SAN & SinoAtrial Node \\
\hline SCD & Sudden Cardiac Death \\
\hline SD & Standard Deviation \\
\hline SDR & Spatial Dispersion of Repolarization \\
\hline SNR & Signal to Noise Ratio \\
\hline SR & Sarcoplasmic Reticulum \\
\hline TM & TropoMyosin \\
\hline TrC & Troponin C \\
\hline TRD & TRiaDin \\
\hline TrI & Troponin I \\
\hline TrT & Troponin $\mathbf{T}$ \\
\hline TTX & TeTrodotoXin \\
\hline $\mathbf{V}_{\mathrm{Eq}}$ & Equilibirum potential (Voltage) of ion \\
\hline $\mathbf{V}_{\mathrm{mbr}}$ & Membrane Voltage \\
\hline V-Fib & Ventricular Fibrillation \\
\hline VSD & Voltage Sensitive Dye \\
\hline V-Tach & Ventricular Tachycardia \\
\hline $\mathbf{V}_{\text {thres }}$ & Threshold potential (Voltage) \\
\hline $\mathbf{V}_{\text {rmp }}$ & Resting Membrane Potential (Voltage) \\
\hline$\Delta \mathrm{KPQ}$ & Mutation in cardiac Na-channel causing a Long QT Syndrome phenotype \\
\hline$\Delta \mathbf{t}_{\text {act }}$ & Global Time of Activation \\
\hline WT & Wild Type (normal) mice \\
\hline$[\text { ion }]_{i}$ & Intracellular ionic concentration \\
\hline [ion $]_{0}$ & Extracellular ionic concentration \\
\hline
\end{tabular}




\section{TABLE OF CONTENTS}

CHAPTER 1 5

INTRODUCTION

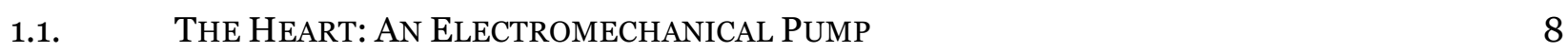

1.1.1. The Functional Syncytium $r$

1.1.2. The Electrical Conduction System $\quad 9$

1.1.3. Biophysics of Excitable Membranes $\quad 10$

1.1.4. Ionic Basis of Electrical Activation $\quad 11$

\begin{tabular}{ll} 
1.1.5. & Excitation-Contraction Coupling (ECC) \\
\hline
\end{tabular}

$\begin{array}{lll}\text { 1.2. } & \text { ANISOTROPY } & 20\end{array}$

1.2.1. Passive Molecular Determinants of Anisotropy 21

1.2.2. The Dystrophin Molecule and the DSMC 23

1.2.3. Active Determinants of Anisotropy 24

1.3. COMPleXity OF ACtivation In The CARDiaC TiSSUE $\quad 25$

1.3.1. Continuous vs. Discontinuous Propagation in Cardiac Muscle 25

1.3.2. Natural Heterogeneity in the Heart 26

1.3.3. Role of Heterogeneity in Cardiac Pathologies $\quad 27$

1.3.4. APD Prolongation and Repolarization Reserves $\quad 28$

1.3.5. Triggered Activity and Ectopic Conduction the Heart Muscle 28

1.3.6. Excitability: A Main Determinant of Conduction in the Heart 30

1.3.7. The Concept of Reentry 31

1.4. ThE CARDIAC SODIUM CHANNEL NAv1.5 31

1.4.1. Structure, Function and Conformational States 32

1.4.2. Modulation of the Cardiac Na-Channel ( $\mathrm{Na}_{\mathbf{v}}$.5) 36

1.4.3. Genetic mutations of Nav1.5: The Long QT Syndrome 37

1.4.4. Spatial Dispersion of Repolarization: An Arrhythmogenic Mechanism 38

1.4.5. Other Mutations of $\mathrm{Na}_{\mathrm{v}} 1.5$ and Heterogeneous Phenotypes 39

1.4.6. Anchoring and Modulating Proteins 40

1.4.7. Pharmacological Interventions: Antiarrhythmic Drugs 42

1.5. PROARRHYTHMIC EFFECTS OF ANTIARRHYTHMIC DRUGS $\quad 43$

1.5.1. CAST I: Increased Mortality Rate with Flecainide 44

1.5.2. Proposed Mechanisms of Flecainide Proarrhythmia 44

1.6. THIS THESIS 46

1.6.1. Conduction Abnormalities in the $m d x$-mouse: A Model of Lower NaV1.5 Availability 47

1.6.2. Implementation and Validation of Analytical Strategies in CV Evaluation in the Murine Heart Using Optical Mapping

1.6.3. Characterization of Spatial-Temporal Heterogeneity Induced by Flecainide Using Clinically Valid Concentrations 
CHAPTER 2 51

EXPERIMENTAL METHODS

2.1. SETUP AND TISSUE PREPARATION

2.1.1. $\quad$ vECG and Pacing Electrodes $\quad 52$

2.1.2. Murine Heart Isolation and Langendorff Perfusion $\quad 52$

2.1.3. Excitation-Contraction Decoupler and VSD Staining 53

2.1.4. 2D Optical Mapping Setup

2.1.5. Animal Models Used in the Current Study and Drugs 55

2.2. ANALYZING THE OPTICAL SIGNAL 56

2.2.1. Signal Processing $\quad 56$

2.2.2. Activation Maps $\quad 56$

2.2.3. ROI and Dispersion Maps $\quad 58$

2.2.4. Analytical Strategies In CV Analysis $\quad 58$

2.2.5. Synthetic Data Used to Simulate Propagation 60

CHAPTER $3 \quad 65$

$\begin{array}{lr}\text { RESULTS } & 65\end{array}$

3.1. CONDUCTION SLOWING IN HEARTS OF MDX-MiCE 66

3.1.1. $\quad$ LSEF: an Anisotropy-Dependent Method 68

3.1.2. PF: Local Calculation of Velocity Wave Fronts 68

3.1.3. AF: An Estimation of Global Conduction Method 69

3.1.4. Deviation from Anisotropy: Failure of Methods' Convergence $\quad 71$

3.1.5. Simulating the Ideal Condition $\quad 74$

3.1.6. Robustness to Noise $\quad 74$

3.1.7. Sensitivity to Heterogeneity in the Milieu of Propagation 75

3.2. FLECAINIDE PROARRHYTHMIA SCALED-DOWN IN THE MDX-MODEL 78

3.2.1. (dF/dt $)_{\max }$ and CV: Indexes of Nav1.5 Availability and Conductance? 78

3.2.2. Symmetry Breaking: A Proarrhythmic Mechanism of Flecainide 89

3.3. $\quad$ EXPATIATED PATHOPHYSIOLOGICAL HETEROGENEITY IN $\triangle$ KPQ 101

3.3.1. Atypical Activation Spread in $\triangle \mathrm{KPQ}$ with Flecainide $\quad 102$

3.3.2. Flecainide Exacerbates Intrinsic Heterogeneity in $\triangle \mathrm{KPQ} \quad 107$

CHAPTER $4 \quad 119$

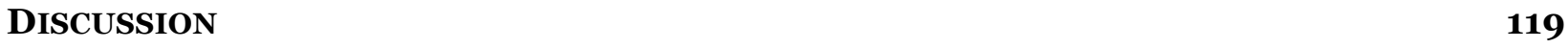

4.1. $\quad$ AP CONDUCTION IN THE MURINE CARDIAC TISSUE 120

4.1.1. Impulse Conduction Slowing in the Dystrophin-Deficient Heart 120

4.1.2. Possible Implications of LM Nav1.5 Loss in $m d x$ : Contribution of LM to the Stability of

Conduction $\quad 121$

4.1.3. Methodological Approaches in Measuring CV 122

4.2. REDUCED EXCITABILITY AND APD PROLONGATION IN MDX-HEARTS 126

4.2.1. Relationship between Nav1.5 Availability, (dF/dt) $\max$ and CV 127

4.2.2. AP Early Phase Prolongation: A Localized Effect of Nav1.5 Loss 130 
4.3. A NOVEL MECHANISM BEHIND FLECAINIDE PROARRHYTHMIA

4.3.1. $\mathrm{APD}_{\mathrm{xx}}$ Prolongation and Increased $A P D_{\mathrm{xx}}$ Dispersion $\left(\triangle \mathrm{APD} \mathrm{D}_{\mathrm{xx}}\right)$ with Flecainide in the Murine Heart

4.3.2. Induction of Bistability by Flecainide in the Murine Heart

4.3.3. Hypothetical Mechanisms Behind Bistability and Arrhythmogenesis

4.3.4. Inflation of Intrinsic Heterogeneity in LQTS3 with Flecainide

139

4.3.5. Concluding Remarks

CHAPTER 5 



\section{Chapter 1}

\section{Introduction}

The words of Jean-Martin Charcot, the Father of Clinical Neurology, still resonate 150 years later, when he spoke of a patient suffering from bulbar amyotrophic lateral sclerosis ${ }^{13}$ : "Yes, the disease that this man has is implacable... It's sad to say, but it's true. However, for the doctor, whether it's sad or not, it's not the issue; the truth is the issue. Let the patient live in illusion to the end. That is fine. It's humane and the best. But the doctor, is it his role to do the same? No, gentlemen, our responsibility is otherwise. Let us keep looking, in spite of everything. Let us keep searching. It is indeed the best method for finding, and perhaps thanks to our efforts, the verdict we will give this patient tomorrow will not be the same as we must give him today'15."

These words haven't lost their strong resonance, possibly because on one hand, Charcot was a physician who understood the vulnerable nature of the human patient, the importance of protecting the quality of life even in the progress of fatal pathologies and the preservation of hope in the treatment of diseases. On the other hand, he was also a scientist, who accentuated the need for the endless search of the truth, emphasized the power of the moral scientific method, as the only way for the prevention, treatment and alleviation of suffering from diseases, which is perhaps humankind's greatest achievement.

Since Charcot, the benefits of applying scientific thought to the practice of medicine have been remarkable. For instance, the progress in the field of Infectious Diseases is enormously tangible at the level of public health, such as the eradication of small pox worldwide with the last reported spontaneous case in 197917, and Polio from the Western Hemispheres around 1990, after the famous work of Salk in developing the oral vaccine ${ }^{18}$. The successful completion of the Human Genome Project in $2003^{19}$ is certainly another global scientific achievement in the field of Genetics. Despite the innumerable advances that were made to improve life expectancy, predicting the pace of scientific research in other multitudinous pathologies is highly uncertain. The diseases of the heart are no exception.

Scientific information, especially genetic and molecular, is in conspicuous expansion and the need to translate this information into improved therapies is pivotal ${ }^{20}$. Over the last century, tools and techniques in the exploration of the heart have undergone tremendous development; from investigating its primary physiological output, i.e. its ability to pump blood rhythmically through contraction and relaxation, to the comprehension of the cellular events and molecular mechanisms and to the appreciation of the structure and function of the various proteins involved in leading up to its almost perfect performance... or deterioration. 
The complexity in approaching cardiovascular physiology was nicely summarized by Berne and Levy21, who wrote: "The precise mathematical expression of the pulsatile flow of blood through the cardiovascular system is insuperable. The heart is a complicated pump and its behavior is affected by a variety of physical and chemical factors. [...] The blood itself is not a simple, homogeneous solution but is instead a complex suspension of red, white corpuscles, platelets and lipid globules dispersed in a colloidal solution of proteins." Despite this inherent complexity of the cardiovascular system, considerable knowledge was gathered starting from simple and elementary mechanical principles, followed by an understanding of the electrophysiology underlying the mechanical contraction $^{22}$, which contributed significantly to the prolongation of life expectancy of patients with cardiovascular diseases.

In 1913, Sir James Mackenzie described angina pectoris in his Diseases of the Heart ${ }^{23}$ as caused by a nervous irritation, where patients are prone to die "during a faint"24. The work of Einthoven in 1895 offered the possibility to record and interpret the electrocardiogram ${ }^{25}$, which gave cardiac symptoms an electrocardiographic component. Several reports in the recent decades confirmed the higher probability of cardiac electrical disturbances, or arrhythmias, occurring in the 15-30min post an acute ischemic event, which are mainly due to abnormal automaticity ${ }^{26}$ (See section 1.3.5). Mines hypothesized about the origin of arrhythmias as early as 1914, and attributed them to a reentry (See section 1.3.7) mechanism ${ }^{27}$, an excitation with a predilection to travel in rings of tissue. He based his hypothesis on Mayer's observation of the Cassiopea xamachana almost a decade earlier, where a contraction wave traveled endlessly in circles secondary to a point stimulus on the jellyfish tissue 28 . Mayer recognized important parameters that facilitate the sustenance of reentry ${ }^{29}$ : the path length or the wavelength, the conduction velocity (CV) and the refractory period (RP), which he referred to as "the time needed for the tissue to rest". The work of Mines on the amphibian heart contributed significantly to the development of the concept of reentry, by emphasizing on the importance of conduction slowing in such a mechanism. He recognized that if the impulse was conducted too rapidly around the ring in one direction, it would return to its original point of initiation before the tissue has recovered from the previous excitation. Whereas, if the conduction is slowed and the refractory period shorter, by the time the impulse returns to its initial position, the tissue would have regained excitability and the impulse will circulate endlessly ${ }^{27}$. It was the work of Janse et al. in 1980, which provided evidence that the sequence of activation of the ectopic beats and the flow of injury currents at the borders between the ischemic and healthy myocardium reinforced a reentrant mechanism ${ }^{30}$ at the early stages after coronary occlusion, which could facilitate the occurrence of fatal cardiac rhythm abnormalities, such as ventricular fibrillation (V-Fib).

V-Fib is a fatal arrhythmia that involves the bulk cardiac tissue. On an ECGii, V-Fib is diagnosed as "aperiodic electrical complexes with irregular beat-to-beat changes in amplitude and morphology" 31. During V-Fib, the ventricular rate is extremely fast $(>500 \mathrm{bpm})$, in consequence the cardiac pumping function is compromised and death ensues in several minutes ${ }^{32}$. Terminating such lethal cardiac activity with a high-energy shock was made possible in 1961, after the introduction of the external defibrillator 33 in specialized cardiac care units. Better understanding of the sequential activation of proteins, cardiac ion channels, involved in the initiation, sustenance and propagation of the electrical impulse, action potential (AP), played a major role in the development antiarrhythmic drugs (AADs). In early 1970s, antiarrhythmic cardiac surgery and electrophysiological interventions using catheter ablation became the second line treatment in patients with drug-refractory arrhythmias.

\footnotetext{
i Angina pectoris -commonly referred to as angina- is chest discomfort due to poor blood flow through the blood vessels in the heart, or myocardial ischemia. Infarction -or scarring- would eventually follow, if no interventional procedure was performed to reopen the closed artery and reperfuse the ischemic tissue.

ii The ECG (acronym for electrocardiogram) is a tracing that represents the heart's electrical activity derived by amplification of the minutely small electrical impulses normally generated by the heart.
} 
The implantable cardioverter defibrillator (ICD) offered a major contribution in prolonging the life expectancy of patients susceptible to spontaneous V-Fib and sudden cardiac death (SCD) since its application $^{34}$ to humans starting 1980. Despite these leading advances in cardiology and electrophysiology in terms of diagnosis, treatment and prevention, SCD remains the most difficult problem to overcome for clinicians and basic medical scientists alike, with more than 300,000 deaths annually in the US alone ${ }^{35}$. Prevention remains inadequate and treatment suboptimal. The efficacy of antiarrhythmic therapy has failed to show commensurate control ${ }^{32}$, except in particular non-fatal settings, and proarrhythmiaiii prevails as the major side effect of antiarrhythmic therapy ${ }^{36}$, 37. This in itself is a sufficient rationale for research in cardiac electrophysiology ${ }^{38}$.

With improvement of management in the setting of acute coronary syndromes, patients are increasingly surviving the acute event. These patients are presenting a few years later with more chronic pathologies and complications that issue from cardiac remodeling secondary to ischemia, such as heart failure, which poses an increasing problem for global healthcare systems ${ }^{39}$ in the Western Hemisphere due to repetitive, costly and prolonged hospitalizations. Currently, more than 14 million people in Europe suffer from heart failure and this number is forecast to reach 30 million by the year $202038,50 \%$ of whom will die from an arrhythmic event ${ }^{40}$.

Unfortunately, the electrophysiological ward is no longer restricted to the older population presented above, it also includes a much younger population of apparently healthy individuals at risk of SCD ${ }^{41}$, where up to $50 \%$ of cases, sudden death is the first and only clinical manifestation of an inherited cardiac disease that had remained undetected by conventional clinical investigations ${ }^{42}$. During the last two decades, considerable progress has been made in the identification of new genes associated with monogenic hereditary arrhythmogenic syndromes, so called "channelopathies" 43 , giving the opportunity to delineate their molecular pathogenesis and identify potential targets for therapeutic interventions ${ }^{44}$. Multidisciplinary integrated type of research is necessary for achieving such a target. Channelopathies remain an active area of research, because the relations between electrophysiological changes in patients with hereditary or chronic cardiac diseases are often far from clear. Identification of genetic mutations and their associated molecular abnormalities, coupled to cellular electrophysiological studies are often hard to extrapolate to the level of the $\operatorname{organ}^{42}$. Hence, functional electrophysiological studies at the organ level (such as optical mapping of intact hearts) and biophysical modeling of the electrical activation are crucial for a complete characterization of diseases ${ }^{45}$. Even with such a combined approach, a discrepancy could be expected, when those findings are extrapolated to the level of the organism.

The work of this thesis falls under integrative cardiac physiology (1.6). By using a multidisciplinary approach, we investigate how targeting the cardiac Na-channel ( $\mathrm{Na} v 1.5)$, whether through genetic mutations or pharmacological interventions, can destabilize the normal electrical propagation in the cardiac tissue. Detailed understanding of propagation instabilities in the whole heart has been impeded by technical difficulties in mapping a beating organ; a particular challenging task in murine electrophysiology, due to the small sized heart and very rapid heart rate. The strength of the work done in this thesis is founded on the methodology used to directly investigate instabilities in conduction and precisely measure the functional parameters at play. Using high spatial-temporal optical mapping, we characterize conduction abnormalities in the mouse heart, which is often used as an in-vitro model of different human cardiac pathologies. This technique is crucial for a more precise and accurate description of macroscopic events occurring in the normal and abnormal heart, leading to a better understanding of fundamental mechanisms involved in arrhythmogenesis.

\footnotetext{
iii Refer to section 1.5 for a detailed description of proarrhyhtmic effects.
} 


\subsection{The Heart: An Electromechanical Pump}

Almost two century ago, Luigi Galvani, later known as the "father of animal electricity" laid down the foundation of electrophysiology 46 by providing evidence for the electrical nature of the "fluid", previously referred to as the "animal spirit", involved in nerve conduction and muscle contraction. He conducted his crucial experiment on September 1786, that was later published in the Commentarius in 1792, where he showed that a dead and "prepared" frog's muscle elicited spontaneous contractions upon joining the nerve and the muscular tissue with a metallic arc. It wasn't before 1856, that Kölliker and Müller ${ }^{47}$ demonstrated the presence of myocardial depolarization and repolarization. Although the conducted experiment was not complicated, the results were puzzling and hard to explain at the time.

\subsubsection{The Functional Syncytium}

The circulatory system is designed to supply the body tissues with oxygen and nutrients in amounts adequate with their metabolic requirements, and to remove carbon dioxide and other waste products for excretion by the lungs and kidneys. This goal is achieved by electrical stimulation that produces sequential ventricular emptying and filling. The heart is delicately designed in order to function with optimal mechanical efficiency, determined by force vectors generated by each sarcomere through active shortening and contraction. The cardiac myocytes are embedded in an extracellular collagen matrix, chiefly made of fibroblasts, a scaffold responsible of maintaining muscle fiber orientation, alignment, ventricular shape and size ${ }^{48}$.

The heart is a four-chambered organ made of two atria and two ventricles. These ventricles are connected to the atria and to the major vessels through valves. The right atrium (RA) receives the deoxygenated blood from the body via the superior and inferior vena cava, empties its content into the right ventricle (RV), which ejects the blood directly into the pulmonary arteries. The blood once oxygenated in the lungs returns to the left side of the heart via the pulmonary veins into the left atrium. The opening of the mitral valve allows the conduit of blood into the left ventricle (LV), which is then distributed back to the body tissues via the aorta. The coronary arteries, which branch from the root of the aorta, supply the heart with blood via the coronary circulation. The atria are thin walled, low-pressure chambers that function mainly as reservoirs facilitating blood entry into their respective ventricles. A continuum of muscle fibers, originating from the fibrous skeleton around the aortic orifice, run toward the apex at the epicardial surface, then pass toward the endocardium as they gradually undergo a $180^{\circ}$ shift rotation to lie parallel to the epicardial fibers, forming the endocardium and papillary muscles ${ }^{49}$.

At the apex the fibers twist and turn inward to form papillary muscles, whereas at the base and around the valve orifices they form a thick and powerful muscle that not only decreases ventricular circumference for ejection of blood but also narrows the atrioventricular valve orifices facilitating valve closure ${ }^{21}$. Ventricular ejection is accomplished by a reduction in circumference in addition to the decent of the base along the longitudinal axis (mainly the RV). The blood is propelled toward the outflow tracts by an earlier contraction of the apex, coupled with an approximation of the ventricular walls ${ }^{21}$. The RV of the adult human heart develops a mean pressure about one seventh that developed by the LV, hence considerably thinner ${ }^{50}$. The heart lies in the middle mediastinum, and is enclosed in the pericardial sac, a $1 \mathrm{~mm}$-thick layer of dense collagen that provides lubrication for the continuous movement of the heart and prevents sudden overdistention of the heart chambers 51 . 
a

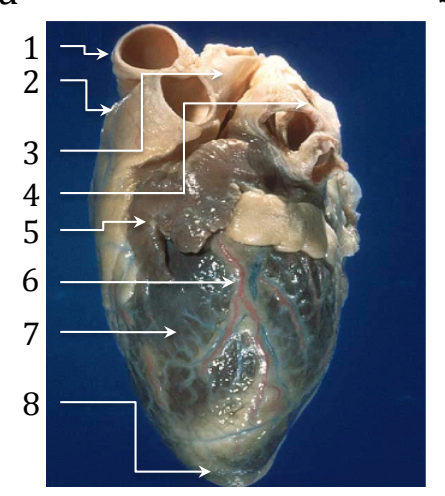

C

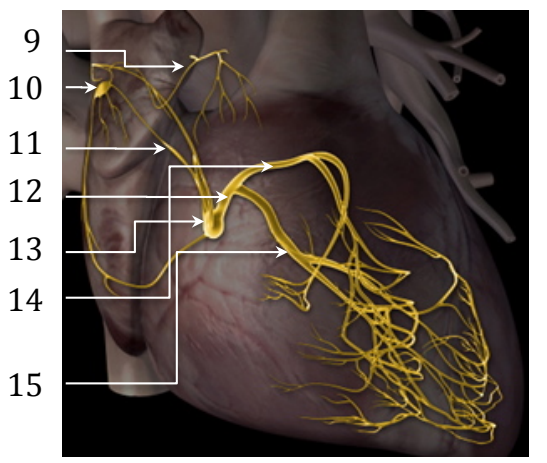

b

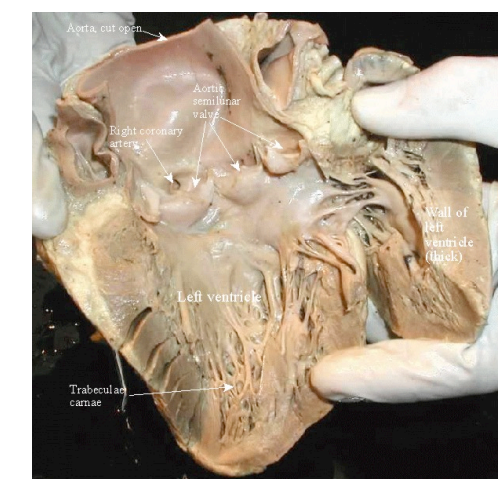

Figure 1. Heart Anatomy and Electrical Conduction System. a. Dorsal aspect of a human heart showing mainly LV1: 1 . L. brachiocephalic vein. 2. Pulmonary trunk. 3. Aorta. 4. Pulmonary veins. 5. L. atrial appendage. $6 . \quad$ L. anterior descending artery. 7. L. ventricular bulk muscle. 8. Apex. b. Heterogeneous interior of LV muscle (endocardium) showing several trabeculae and the papillary muscles 4 c. Main components of electrical conduction system $^{5}$ : 9. Interatrial pathway. 10. SAN. 11. Internodal pathway. 12. His Bundle. 13. AVN. 14. Right bundle branch. 15. Left bundle branch. Human heart weight varies between $300-350 \mathrm{~g}$ with apex-base distance $\sim 110 \mathrm{~mm}$ and the largest transverse dimension $\sim 75 \mathrm{~mm}^{8}$. Pictures taken from references as noted.

The sequential contraction and activation of atria and ventricle occur in each cardiac cycle: the isovolumic contraction coincides with the abrupt rise in ventricular pressure on the pressurevolume curve $\mathrm{e}^{52}$, known as the pre-ejection tension. This phase is followed by a rapid and reduced ejection, an isovolumic post-ejection relaxation, characterized by a precipitous fall in ventricular pressure that signals the beginning of ventricular diastole, which is followed by a rapid filling phase and diastesis before the next cycle starts again. Major intrinsic mechanisms that influence the cardiac pumping function are the Frank-Starling effect ${ }^{53}$ and the length-dependent regulation of stroke volumeiv54, as well as the treppe- or Bowditch effect55 (these mechanisms will not be developed here, check references for more details). This description, although still considered correct, adopts simple assumptions ${ }^{56}$ regarding ventricular function: it implicitly suggests for instance, that the cardiac muscle is homogeneous in such a way that all cardiac fibers contract and relax simultaneously. It fails to recognize the complex multilayer muscle architecture underlying the fairly heterogeneous contractile behavior, such as narrowing, twisting and regional myocardial straining56.

\subsubsection{The Electrical Conduction System}

The cardiac muscle generates propagating electrical impulses, action potentials or AP (Figure 2a). Hence it's classified in the category of excitable tissues, together with skeletal muscles and nerves. Additional intrinsic properties to the cardiac tissue are rhythmicity and automaticity, i.e. the ability to regularly and spontaneously initiate APs. Under normal sinus rhythm, the propagating wave of electrical excitation is initiated in the pacemaker cells of the sinoatrial node (SAN) situated in the

\footnotetext{
iv The stroke volume is defined as the volume of blood ejected from the LV per beat. The cardiac output is defined as the volume of blood ejected from the LV in a minute. Hence the cardiac output is the product of stroke volume and heart rate (beats/min)
} 
groove where the superior vena cava joins the RA ${ }^{57}$ (Figure 1c). From the SAN, the cardiac impulse travels radially throughout the $\mathrm{RA}^{58}$ along ordinary atrial cells at $\mathrm{CV} \sim 1 \mathrm{~m} . \mathrm{s}^{-1}$. A special pathway, the anterior interatrial myocardial band (or Bachmann's bundle) conducts the impulse from the SAN to the left atrium. The impulse reaches the atrioventricular node (AVN), before gaining access to the His Bundle, the specialized conducting system of the ventricles. Several features of the AV conduction are of physiological and clinical significance ${ }^{59}$. The principal delay in the passage of the impulse from the atria to the ventricles occurs in the AVN, allowing for optimal ventricular filling during atrial contraction. The His Bundle passes subendocardially for about $1 \mathrm{~cm}$ and then divides into right and left bundle branches. The left bundle further divides into thin anterior and thick posterior divisions. The bundle branches ultimately subdivide into a complex network of fast conducting Purkinje fibers ${ }^{60}$. The excitation travels through the bulk muscles almost coherently in a quasiplanar fashion, triggering cardiac contraction and synchronizing its blood pumping ability.

The unique property of the cardiac muscle to initiate rhythmically its own contractions, a function that is primarily associated with SAN cells, is known as the central dogma of automaticity. The failure of the SAN to entrain the heart at the frequency of its pacemaker cells facilitates the triggering of ectopic beats (secondary or accessory pacemakers), at slower rates than the SAN. The rate of impulse initiation due to automaticity of cells in the SAN is sufficiently rapid that potentially automatic cells elsewhere in the heart are excited by propagated impulses before they can depolarize spontaneously to threshold potential 61 . This inhibition is called overdrive suppression ${ }^{62}$, which means driving a pacemaker cell faster than its intrinsic spontaneous rate. A shift in the site of impulse generation to a region other than the SAN would be expected to occur when the sinus rate falls considerably below the intrinsic rate of the subsidiary pacemakers having the capabilities for normal automaticity ${ }^{63}$. The sequence of accessory pacemakers starts with the AV nodal cells that will fire at a rate $<60 \mathrm{bpm}$, followed by Purkinje fibers (at a rate $<40 \mathrm{bpm}$ ) ${ }^{64}$. The latter is considered usually not sufficient to allow the heart to pump an adequate cardiac output. The Ionic Basis of Electrical Activation is detailed in section 1.1.4.

\subsubsection{Biophysics of Excitable Membranes}

The whole organ cardiac dynamics can be traced back to cellular events regulating intracellular ion homeostasis 65 . Cells, by virtue of their compartmentalization, have intracellular and extracellular environments with many vital biochemical processes taking place in or on membrane surfaces. For cellular processes to run efficiently inside the cell, cells have developed the appropriate energy producing tools (such as ATP hydrolysis) to use the chemistry of their environment to their benefit ${ }^{66}$. Since ions cannot readily cross the lipid bilayer, cells make use of the gradient of electrical energy as a key driving force in the movement of salt (therefore water), an essential element in the signaling processes, and ultimately the basis of all cognitive processes ${ }^{67}$. Na-K-ATPase, a ubiquitously expressed transmembrane protein, creates this gradient by hydrolyzing ATP, therefore maintains the large outwardly directed $\mathrm{K}^{+}$gradient and large inwardly directed $\mathrm{Na}$ gradient, a hallmark of all animal cells. Another physiological parameter that helps maintain this electrical gradient is an intrinsic property of the bilayer: permselectivity 68 , which means that cell membranes display different degrees of permeability to different ions establishing an uneven distribution of ions across the membrane. Negatively charged, nonpermeant proteins present predominantly in the intracellular compartment, are large molecular weight substances and therefore do not cross the plasma membrane. In consequence, they will attract positively charged ions and repel negatively charged ones. If diffusion of positive ions into the cell were indefinite, it would eventually alter ion, volume and $\mathrm{pH}$ homeostasis, leading to cell destruction ${ }^{69}$. 
With all the above forces at play, the cell establishes a Gibbs-Donnan equilibrium when the altered distribution of cations and anions results in an electrochemical equilibrium for the major principal ions moving in and out of the cell ${ }^{70}$. Under resting conditions, since the cardiac cell is categorized as excitable, the plasma membrane is highly permeable to $\mathrm{K}^{+}$. The injection of a strong enough current will cause the cardiac cell to respond by eliciting an AP, the result of sequential transient changes of membrane permeability to the principal ions $\mathrm{Na}^{+}, \mathrm{K}^{+}, \mathrm{Ca}^{2+}$ and $\mathrm{Cl}$. The equilibrium potential for each of the major 3 ions with a significant influence in bioelectrical phenomena $\left(\mathrm{Ca}^{2+}\right.$ to a certain extent in heart tissues) and it approximates as the following: $+62 \mathrm{mV}$ for $\mathrm{Na}^{+},-94 \mathrm{mV}$ for $\mathrm{K}^{+},+120 \mathrm{mV}$ for $\mathrm{Ca}^{2+}$ and $-80 \mathrm{mV}$ for $\mathrm{Cl}^{-}$. $\mathrm{V}_{\mathrm{Eq}}$ of $\mathrm{K}^{+}$and $\mathrm{Na}^{+}$set boundary conditions for the electrical potentials across the membrane. A cardiac tissue cannot normally be more negative than $-94 \mathrm{mV}$ or more positive than $+62 \mathrm{mV}$, simply because there are no relevant chemical gradients that could produce larger potential differences ${ }^{67}$. Any manipulation of the concentration gradients (experimentally or pathologically) will ultimately affect the equilibrium potential of that particular ion. When multiple permeating ions are at play, then the resulting resting membrane potential $V_{\text {rmp }}$ can be calculated using the Goldman-Hodgkin-Katz equation 71, 72:

$$
V_{r m p}=-\frac{R T}{F} \ln \left(\frac{p_{K}\left[K^{+}\right]_{i n}+p_{N a}\left[N a^{+}\right]_{\text {in }}+p_{C l}\left[C l^{-}\right]_{\text {in }}}{p_{K}\left[K^{+}\right]_{\text {out }}+p_{N a}\left[N a^{+}\right]_{\text {out }}+p_{C l}\left[C l^{-}\right]_{\text {out }}}\right)
$$

$p_{\text {ion }}$ is relative membrane permeability for that particular ion

[ion] is the concentration of the ion species inside and outside the cell

The permeability of the membrane to any of the ions can change dramatically upon changes in membrane potential, by alterations in the conducting properties of voltage dependent transmembrane pore-forming proteins, known as channels. Any process that abruptly changes the membrane potential beyond a critical value threshold ( $\mathrm{V}_{\text {thres }}$ ) will induce an AP capable of propagation. Fundamental properties of excitable tissues can be summarized as follows:

a. The resting excitable state, where minor perturbations of the membrane potential will decay without pushing the system toward the excited state.

b. Perturbations strong enough to cross $V_{\text {thres }}$ will push the system to display a characteristic transient behavior producing an AP, through a process called depolarization.

c. The system will return to its initial resting state through repolarization, during which two particular divisions of time take place consecutively: the first where the system is absolutely non-excitable (effective refractory period-RP); the second where it displays depressed responses due to reduced excitability (relative RP)

\subsubsection{Ionic Basis of Electrical Activation}

At the level of cardiac ventricular myocyte, the fast response is triggered by the activation of voltage-gated $\mathrm{Na}$-channels, $\mathrm{Na}_{\mathrm{v}} 1.5$. The rapid depolarization phase (phase 0 ) is almost exclusively mediated by the rush of the $\mathrm{Na}^{+}$ions into the cell, by virtue a sudden brief increase in membrane permeability to $\mathrm{Na}^{+73}$. The detailed concerted mechanism of $\mathrm{Na}_{v} 1.5$ activation and inactivation is thoroughly explained in section 1.4.1. An important difference in the general morphology of the cardiac AP, as compared to the skeletal and nervous AP, is a plateau (phase 2) that separates the fast depolarization from repolarization (phase 3). The plateau is physiologically significant to allow for a substantial enough increase of intracellular $\mathrm{Ca}^{2+}$ to mediate contraction ${ }^{74}$. The durations of both Phase 2 and 3 provide control of mechanical contraction, prevent premature arrhythmogenic excitation and modulate AP time course and duration. This section will explore the ionic mechanisms and cellular complexity involved in generating the ventricular AP, and the electrophysiological heterogeneity that exists between different layers of the ventricular wall. Excitation-contraction coupling is dealt with in section 1.1.5 and AP propagation in section 1.3.1. 
a

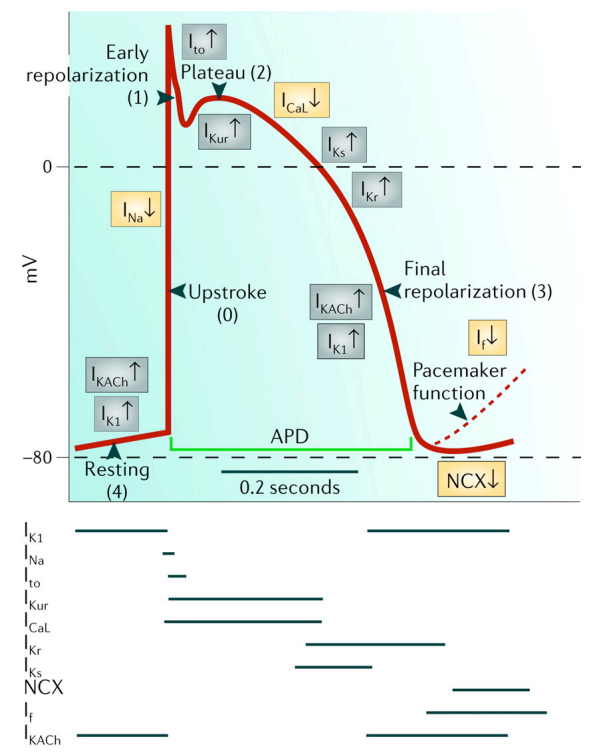

b

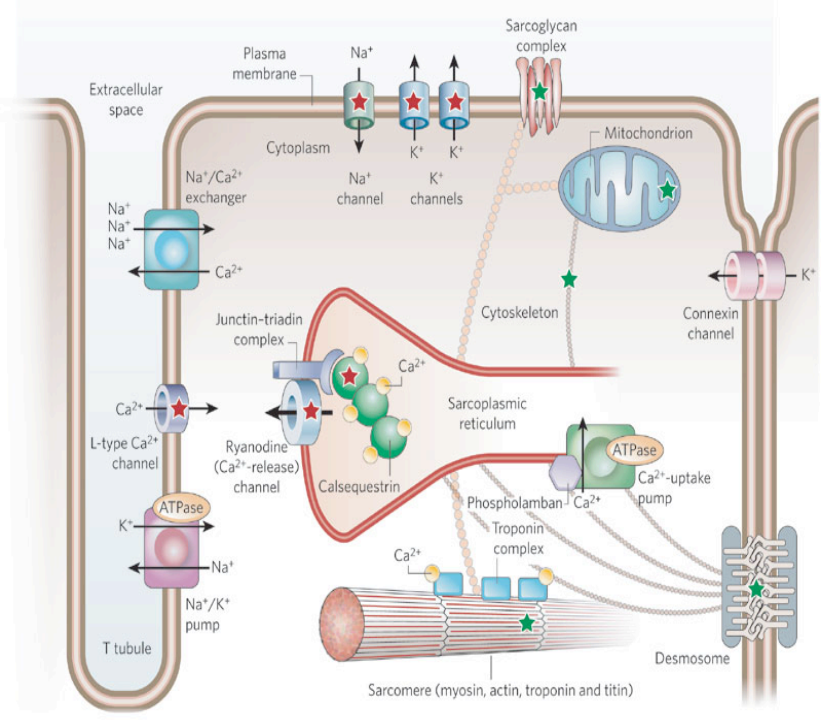

Copyright (c) 2006 Nature Publishing Group Nature Reviews | Drug Discovery

Figure 2. Ventricular AP and ventricular myocyte. a. Membrane current generating a normal AP75. The 5 different phases of the AP: resting (4), upstroke (0), early repolarization (1), plateau (2) and final repolarization (3). The major inward $\mathrm{I}_{\mathrm{Na}}, \mathrm{I}_{\mathrm{Ca}}$ and $\mathrm{I}_{\mathrm{f}}$ currents are shown in yellow boxes. The outward currents are illustrated in grey boxes. The Na-Ca exchanger (NCX) pump is electrogenic: it mediates in-/out-ward currents. b. Cellular machinery at work: proteins, intracellular organelles, ionic movement involved in excitation-contraction coupling ${ }^{76}$. The ventricular cell is kept at hyperpolarized state with the efflux of inward rectifier IK1 current, and the Na-K-ATPase maintains the ionic gradient across the membrane. The propagated action potential reaches the ventricular myocyte initially through ions that slowly depolarize the membrane potential to threshold value, opening the $\mathrm{Na}_{\mathrm{v}} 1.5$, creating the upstroke and triggering the opening of L-Type voltage gated Ca-channels ( $\mathrm{I}_{\mathrm{Ca}, \mathrm{L}}$ ) and K-channels ( $\mathrm{I}_{\mathrm{Kur}}$ ). During the plateau, CICR increases the intracellular calcium almost 100 folds, via RyR2. $\mathrm{Ca}^{2+}$ binds to troponin $\mathrm{C}$, and initiates contraction. $\mathrm{K}^{+}$conductivity increase ends the plateau phase ( $\mathrm{IKr}_{\mathrm{K}} \mathrm{IKs}_{\mathrm{K}}$ ) and initiates cellular relaxation, with synchronized reuptake of $\mathrm{Ca}^{2+}$ into the SR mainly via Ca-pump and NCX. Phosphorylation of troponin I inhibits $\mathrm{Ca}^{2+-b i n d i n g ~ t o ~ t r o p o n i n ~ C, ~ w h i c h ~ p e r m i t s ~}$ tropomyosin blocking of acting-myosin interacting site and sarcomere relaxation. Red stars corresponds to proteins when mutates cause primary arrhythmia syndromes. Green stars correspond to multiple genes when involved cause cardiomyopathies that often involved arrhythmia. Pictures taken from references as noted.

Ions channels that sequentially mediate the AP provide pathways of low resistance to the passage of ionic species across the membrane. Unlike the Na-K-ATPase that hydrolyses ATP for ion transport energy, the activated channels permit passive movement of ions, resulting in ion flux that is characteristically rapid $\left(10^{7} \text { ions/s }\right)^{77}$, 78 . By convention, inward currents are the equivalent to the movement of positive charges into the intracellular milieu. Therefore, inward currents are generally depolarizing currents ${ }^{79}$ (fast inward $\mathrm{I}_{\mathrm{Na}}$ in ventricular myocytes and $\mathrm{I}_{\mathrm{Ca}}$ in nodal cells). The movement of positive charges (carried mainly by $\mathrm{K}^{+}$) to the extracellular milieu defines outward currents. $\mathrm{I}_{\mathrm{Ks}}$ is the major outward current opposing the influx of $\mathrm{Ca}^{2+}$ during the plateau ${ }^{80}$. Cl- influx into the cell is also considered, based on the previous definition, an outward repolarizing current.

Ohmic currents obey Ohm's law $\left(\Delta \mathrm{V}_{\mathrm{mbr}}=\mathrm{I}_{\mathrm{ion}} \mathrm{R}\right)$, which states that a current is linearly coupled to the membrane voltage and the resistance is voltage- and time-independent ${ }^{81}$. $\mathrm{I}_{\mathrm{Ks}}$, the slowly activating component of the $\mathrm{I}_{\mathrm{K}}$ current during repolarization displays ohmic properties. The rapid component of the $\mathrm{I}_{\mathrm{K}}\left(\mathrm{I}_{\mathrm{Kr}}\right)$ displays rectification properties ${ }^{82}$, 83. Rectification describes the dependence of 
resistance on membrane voltage, with outward and inward characteristics ${ }^{84}$. Outward rectification occurs when the membrane conducts an outward flow of current at voltages more positive than the equilibrium potential of the ion $V_{\mathrm{Eq}(i o n)}$, more easily than it does an inward one at potentials negative to $\mathrm{V}_{\mathrm{Eq}(\mathrm{ion})^{71}}$. Inward rectification involves easier conductance of an inward current at membrane

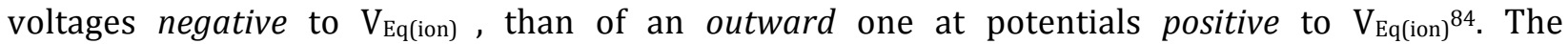
rectification behavior was first described in skeletal muscles ${ }^{85}$ and $\mathrm{K}^{+}$currents displaying this property were originally known as anomalous currents, they were characteristically different from the repolarizing $\mathrm{K}^{+}$current described in the giant squid axon by Hodgkin and Huxley ${ }^{86}$. Unlike voltage-gated K-channels $\left(\mathrm{K}_{\mathrm{v}}\right)$, channels with inward rectification properties $\left(\mathrm{K}_{\mathrm{ir}} 2 . \mathrm{x}\right)$ do not depend on $\mathrm{V}_{\mathrm{mbr}}$ only, but also on the difference between $\mathrm{V}_{\mathrm{mbr}}$ and $\mathrm{V}_{\mathrm{Eq}, \mathrm{K}}$, as $\left[\mathrm{K}^{+}\right]_{\mathrm{o}}$ is changed ${ }^{31,87}$. They play a major role in maintenance of the $\mathrm{V}_{\mathrm{rmp}}$ and control AP duration (APD), through their characteristic gating kinetics ${ }^{80}$. In channels mediating $\mathrm{I}_{\mathrm{K} 1}$, the main gating particles are intracellular polyamines and $\mathrm{Mg}^{2+}$, which are now widely accepted as the primary mechanism in the inward rectification property of those channels 88,89 .

- AP Upstroke (Phase 0). During electrical diastole, $V_{\mathrm{rmp}}$ is maintained by $\mathrm{I}_{\mathrm{K} 1}$ via inwardly rectifying channels ${ }^{90}$. Voltage-gated channels have charges or dipoles that sense changes in membrane potential and respond with changes in conformation. These conformational changes generate small currents, gating currents ${ }^{91}$ that precede the voltage dependence conductance. When $\mathrm{Na}^{+}$influx is strong enough to counteract the effects of $\mathrm{K}^{+}$efflux, $\mathrm{V}_{\mathrm{mbr}}$ slightly depolarizes to $\mathrm{V}_{\text {thres}}$, increasing the opening probability of $\mathrm{Na}_{\mathrm{v}} 1.5^{92}$. Once $\mathrm{V}_{\text {thres }}(\sim-$ $65 \mathrm{mv}$ ) is reached, $\mathrm{Na}^{+}$membrane conductance abruptly rises, depolarizing the membrane to $\sim+30 \mathrm{mV}$. The fast-inward current $\left(\mathrm{I}_{\mathrm{Na}, \mathrm{i}}\right)$, mediated by $\mathrm{Na}_{\mathrm{v}} 1.5$, generates the AP upstroke, then quickly inactivates. The rapidity of depolarization is responsible for the fast response that will ensure whole myocardium depolarization in less than $20 \mathrm{~ms}$, a nearly synchronous excitation followed by contraction ${ }^{93}$. The maximum rate of rise of the upstroke $(\mathrm{dV} / \mathrm{dt})_{\max }$ reflects non-linearly the magnitude of the total fast-inward current ${ }^{94}$. Purkinje fibers, which have the highest $(\mathrm{dV} / \mathrm{dt})_{\max } \sim 750-1000 \mathrm{~V} / \mathrm{s}^{95}$, also have the highest density of $\mathrm{Na}_{\mathrm{v}} 1.5$ channels than do ventricular myocytes with $(\mathrm{dV} / \mathrm{dt})_{\max } \sim 200-300 \mathrm{~V} / \mathrm{s}$. Factors that decrease $\mathrm{Na}_{\mathrm{v}} 1.5$ availability, decrease $\mathrm{I}_{\mathrm{Na}, \mathrm{i}}$ magnitude, hence expected to decrease $(\mathrm{dV} / \mathrm{dt})_{\max ^{96}}$. In pathological conditions, where $V_{\text {rmp }}$ is set to more depolarized potentials, suprathreshold stimuli will elicit depressed fast responses, due to lower availability of $\mathrm{Na}_{v} 1.5$ in their resting excitable states. For instance, crushing injuries rapidly releases $\mathrm{K}^{+}$into the blood from damaged tissues, increasing $\left[\mathrm{K}^{+}\right]_{\mathrm{o}}$ from its lower value of $\sim 4 \mathrm{mMol}$ (normally) to a few mmol higher, which can have drastic effects on the $\mathrm{V}_{\mathrm{rmp}}$, as predicted by the Goldman (equation in section 1.1.3). In acute ischemia, Na-K-ATPase failure contributes to the increase in the $\left[\mathrm{K}^{+}\right]_{\text {o, }}$ exacerbated by both the low $\left[\mathrm{K}^{+}\right]_{\mathrm{o}}$ starting value and low interstitial fluid volume in confined organs such as the heart ${ }^{97}$. If $\mathrm{V}_{\text {rmp }}$ is depolarized to $-55 \mathrm{mV}$, almost all $\mathrm{Na}_{\mathrm{v}} 1.5$ will not open and the tissue is no longer excitable ${ }^{98}$. As discussed later in section 1.3.6, $(\mathrm{dV} / \mathrm{dt})_{\max }$ and AP amplitude are important determinants of CV throughout the myocardium ${ }^{99}$.

- Early repolarization (Phase 1). The AP upstroke is followed by a phase, which repolarizes the membrane to $\sim+15 \mathrm{mV}$. This rapid repolarization is mainly due to activation of the transient outward $\mathrm{K}^{+}$current $\mathrm{I}_{\mathrm{K}, \text { to }}$ or $\mathrm{I}_{\mathrm{to}, 1}$, the calcium dependent inward $\mathrm{Cl}^{-}$current $\mathrm{I}_{\mathrm{Ca} . \mathrm{Cl}}$ or $I_{t o, 2}$, and the time-dependant inactivation of $\mathrm{Na}_{v} 1.5$. Prominent $I_{t o}$ currents were recorded from ventricular cells of many species, including mice, rabbits, dogs and humans 100 . A notable exception is the guinea pig, where $I_{\text {to }}$ is absent or practically non-measurable 101,102 . $\mathrm{I}_{\mathrm{to}, 1}$ is rapidly activating (time constant $10 \mathrm{~ms}$ ), calcium-independent and displays outward rectification properties ${ }^{7}$. It is further composed of at least two functionally different components: fast $\mathrm{I}_{\mathrm{to}, \mathrm{f}}$ and slow $\mathrm{I}_{\mathrm{to}, \mathrm{s}}$, which mainly differ in their inactivation kinetics. Their 
recovery time constants are on the order of tens of ms and thousands of ms, respectively. $\mathrm{I}_{\mathrm{to}, \mathrm{f}}$ is the principal subtype expressed in the human atria, whereas the ventricles express both types. $\mathrm{I}_{\text {to }}$ is more prominent in myocardial regions with shorter APD, such as the epicardium, $\mathrm{RV}$, and septum with a spike and dome configuration. In the endocardium, $\mathrm{I}_{\mathrm{to}}$ is weakly expressed and phase 1 is practically negligible ${ }^{103}$. $\mathrm{I}_{\mathrm{to}, \mathrm{f}}$ channels contribute significantly to the rapid repolarization in murine ventricular myocytes ${ }^{104}$, a property deemed to be necessary in order to maintain the very high resting heart frequency $(>11 \mathrm{~Hz})$ in these animals ${ }^{105}, 106$. This electrical diversity arises from combined effects of spatial tissue gradients of gene expression, biophysical channel gating mechanisms and second messenger systems ${ }^{107}$. $\alpha-$ adrenergic stimulation reduces $I_{\text {to }}$ in human myocytes through PKA-dependent phosphorylation. In heart failure, this current is also reduced and seems to be associated with APD prolongation ${ }^{108}$. $\mathrm{I}_{\mathrm{to}, 2}$, unlike $\mathrm{I}_{\mathrm{to}, 1}$, is a calcium-dependant $\mathrm{Cl}^{-}$current and insensitive to 4-aminopyridine ${ }^{109}$. The effects of $\mathrm{I}_{\mathrm{to}, 2}$ are variable: at positive membrane potentials, $\mathrm{Cl}$ - is driven into the cell by virtue of its electrostatic and diffusional forces, driving the membrane potential to more negative values. Intracellular $\mathrm{Ca}^{2+}$ homeostasis plays a key role in determining the contribution of $\mathrm{I}_{\mathrm{to}, 2}$ to the total $\mathrm{I}_{\mathrm{to}}$ repolarizing current, since its activation correlates with $\mathrm{Ca}^{2+}$ release from the sarcoplasmic reticulum (SR), which in turn is triggered by the influx of $\mathrm{Ca}^{2+}$ through L-type channels ${ }^{110}$.

$\circ \quad$ The plateau (Phase 2). This phase reflects a delicate balance between inward and outward currents. Inactivation of $\mathrm{I}_{\mathrm{Na}}$ and significant decrease in $\mathrm{I}_{\mathrm{K} 1}$ conductance increases membrane resistance. If $\mathrm{I}_{\mathrm{K} 1}$ conductance during phase 2 were equal to the one in phase 4 , the efflux of $\mathrm{K}^{+}$would greatly exceed the influx of $\mathrm{Ca}^{2+}$ and a plateau phase would be unsustainable 21 . Opening of the voltage-dependent calcium channels is reflected by a gradual increase in $\mathrm{Ca}^{2+}$ conductance, which immediately begins after the depolarizing upstroke. Two types of transmembrane $\mathrm{Ca}^{2+}$ channels co-exist in the heart ${ }^{111}$. The less abundant $\mathrm{T}$-type ( $\mathrm{T}$ for transient) channels activate at $\mathrm{V}_{\mathrm{mbr}} \sim-70 \mathrm{mV}$, then inactivate very quickly. $\mathrm{I}_{\mathrm{Ca}, \mathrm{T}}$ is restricted to pacemaker, atrial and Purkinje cells ${ }^{7}$. $\mathrm{I}_{\mathrm{Ca}, \mathrm{L}}$ (L for long lasting; L-type channels are also known as Dihydropyridine receptors or DHPR) is present in all cardiac cells and activates at more positive potentials $(\sim-25 \mathrm{mV})$. It provides a depolarizing inward current that supports the AP plateau against the repolarizing outward $\mathrm{K}^{+}$currents. Even when it activates with the upstroke, $\mathrm{I}_{\mathrm{Ca}, \mathrm{L}}$ reaches its peak value at a rate of two orders of magnitude less than the fast inward $\mathrm{I}_{\mathrm{Na}}$. Hence its peak contribution to the rising upstroke is considered negligible ${ }^{112}$. At depolarized potentials, the $\mathrm{I}_{\mathrm{Ca}, \mathrm{L}}$ recovers from inactivation very slowly $(>100 \mathrm{~ms} \text { at } 0 \mathrm{mV})^{77}$ : it's the dome shape of the calcium curve that contributes to the maintenance of the plateau. $\mathrm{I}_{\mathrm{Ca}, \mathrm{L}}$ inactivation is time-, voltage- and $\left[\mathrm{Ca}^{2+}\right]_{\mathrm{i}}$ dependent ${ }^{\mathrm{v}}$, the latter is by far predominant ${ }^{113}$. The principal function of the $\mathrm{I}_{\mathrm{C}, \mathrm{L}}$ is triggering $\mathrm{Ca}^{2+}$ release from the $\mathrm{SR}$, to provide enough intracellular free $\mathrm{Ca}^{2+}$ for contraction to occur (See section 1.1.5). $\mathrm{I}_{\mathrm{Ca}, \mathrm{L}}$ can be modulated by various factors, hence influencing cardiac contractility. Cathecholamines interact with $\beta$ receptors, which increases intracellular cAMP, lead to an increase in $\mathrm{I}_{\mathrm{Ca}, \mathrm{L}}$ magnitude, without affecting $\mathrm{I}_{\mathrm{Ca}, \mathrm{T}}$.

In addition to $\mathrm{I}_{\mathrm{Ca}, \mathrm{L}}$, repolarization is delayed by two other inward Na-mediated currents: $\mathrm{I}_{\mathrm{NCX}}$ and the late or persistent Na-current $\mathrm{I}_{\mathrm{Na} \text {.. }}$. The Na-Ca-exchanger pump (NCX) contributes to the plateau by generating a net inward Na-current. By virtue of its stoichiometry $\left(\mathrm{Na}^{+}: \mathrm{Ca}^{2+}=3: 1\right)$ and its electrogenicity, the direction of exchange adopted by the pump is coupled to combined $V_{\mathrm{Eq}, \mathrm{NCX}}$ of the involved ions and $V_{m b r}{ }^{114}$. At resting states, the $V_{\mathrm{Eq}, \mathrm{NCX}}$ approximates to $-40 \mathrm{mV}$, a more positive than the $\mathrm{V}_{\mathrm{mbr}}$. Hence the pump operates in its direct mode, producing a net inward $\mathrm{Na}^{+}$current and

v $\mathrm{Ca}^{2+}$-Calmodulin complex modulation of the $\mathrm{I}_{\mathrm{Ca}, \mathrm{L}}$ (refer to section 1.1.5) 
extruding $\mathrm{Ca}^{2+}$. During the brief phase 0 and $1, \mathrm{~V}_{\mathrm{mbr}}$ is depolarized to positive values, exceeding $\mathrm{V}_{\mathrm{Eq}, \mathrm{NCX}}$, which results in a brief outward current with NCX operating in the indirect mode ${ }^{114}$. The increase in $\left[\mathrm{Ca}^{2+}\right]_{\mathrm{i}}$ during phase 2 pushes the $\mathrm{V}_{\mathrm{Eq}, \mathrm{NCX}}$ to more positive values, reverting the pump to the direct mode, thereby generating a net inward current ${ }^{115,116 .}$

In ventricular and Purkinje fibers, inactivation of $\mathrm{Na}_{\mathrm{v}} 1.5$ is fast, however possibly incomplete ${ }^{93}$. A relatively minuscule current $\left(<0.5 \%\right.$ of peak $\mathrm{I}_{\mathrm{Na}, \mathrm{i}}$ current ${ }^{117}$ under normal conditions), known as the window current, is present due to reactivation of a very small fraction of $\mathrm{Na}_{\mathrm{v}} 1.5$ at fairly depolarized potentials ${ }^{118}$. Under physiological conditions, this late current is restricted and confined to a narrow range of voltages, with a hampered effect on the AP repolarization dynamics ${ }^{119}$. This positive current flows at positive $V_{m b r}$, where the Hodgkin-Huxley model predicted zero current. At the molecular level, the fact that a population of $\mathrm{Na}_{\mathrm{v}} 1.5$ is open in this voltage window, in the absence of pathological conditions, implies the finite possibility that inactivation could be a reversible process ${ }^{120}$, allowing channels to open after they have inactivated. However under pathological conditions, the sodium current could become non-inactivating or persistent $\left(\mathrm{I}_{\mathrm{Na}, \mathrm{p}}\right)$ and flows throughout the $\mathrm{AP}^{121,122}$, lasting 50-100 times longer than the upstroke time, which could contribute substantially to Na loading during each cardiac cycle ${ }^{93}$. Under pathologically acquired (hypoxia ${ }^{123}$, heart failure ${ }^{124}$ ) or congenital conditions ${ }^{125}$ (such as Long QT Syndrome 3 or LQTS3vi), amplification and sustenance of this current has been strongly implicated in arrhythmogenesis, particularly since $\left[\mathrm{Na}^{+}\right]_{i}$ homeostasis is tightly coupled to $\mathrm{Ca}^{2+}$ regulation ${ }^{126,127}$. Linkage between this sustained current, AP prolongation and proarrhythmic activity has been validated in quantitative modeling128. Increased $\left[\mathrm{Na}^{+}\right]_{\mathrm{i}}$ has also been shown to augment a Na-activated K-current or $\mathrm{I}_{\mathrm{K}(\mathrm{Na})}$ and has been suggested to affect APD under conditions of $\left[\mathrm{Na}^{+}\right]_{\mathrm{i}}$ overload ${ }^{126,129}$. $\mathrm{I}_{\mathrm{Na}, \mathrm{K}}$ (the Na-K-ATPase current) contributes slightly to the AP plateau by extruding $\mathrm{Na}^{+}$ions that entered the cell during the upstroke, partly due to its voltage-dependence property ${ }^{130}$.

The delayed rectifier $\mathrm{K}^{+}\left(\mathrm{I}_{\mathrm{K}}\right)$ is generally considered the dominant outward current. The $\mathrm{I}_{\mathrm{K}}$ conductance increases gradually throughout phase $2^{131}$. As long as the efflux of $\mathrm{K}^{+}$is balanced by the influx of $\mathrm{Ca}^{2+}$, the plateau persists. The three components of the $\mathrm{I}_{\mathrm{K}}$ (ultra rapid: $\mathrm{I}_{\mathrm{Kur}}$, rapid: $\mathrm{I}_{\mathrm{Kr}}$, slow: $\mathrm{I}_{\mathrm{Ks}}$ ) activate slowly (compared to $\mathrm{I}_{\mathrm{Na}, \mathrm{i}}$ ) over a range of several hundreds of $\mathrm{ms}$, at voltages that prevail toward the end of phase $0^{132}$. Their deactivation is sufficiently slow that their contribution to the AP is mainly in restoring repolarization and ending the plateau ${ }^{82} \mathrm{I}_{\mathrm{Kur}}$, highly expressed in atrial cells (but not in ventricular or Purkinje fiber), is rapidly activating, non-inactivating current and is believed to shape the shorter APD duration in atrial cells ${ }^{133}$. $\mathrm{I}_{\mathrm{Kr}}$ is the rapid delayed rectifier and increases faster than $\mathrm{I}_{\mathrm{Ks}}$ at the initial phase of the AP, however reaches a smaller magnitude lower peak current amplitude) than $I_{K s}$, due to its inward rectification properties that prevail at depolarized potentials. It's heterogeneously expressed in the heart, with higher density in the LA and endocardium 134,135 . As the membrane slowly repolarizes, $\mathrm{I}_{\mathrm{Kr}}$ curve reaches its local maximum due to decreased inward rectification. Compared with $\mathrm{I}_{\mathrm{Kr}}$, $\mathrm{I}_{\mathrm{Ks}}$ increases slowly due to slower activation kinetics. However, it reaches a higher peak than $\mathrm{I}_{\mathrm{Kr}}$ during much of the plateau, due to its linear (non-rectifying) conductance, greater density of $\mathrm{I}_{\mathrm{Ks}}$ channel in the membrane ${ }^{112}$, and its dependence on $\left[\mathrm{Ca}^{2+}\right]_{i}$ increase via a calmodulinvii $(\mathrm{CaM})$ modulating process ${ }^{132}$. It's ubiquitously expressed, however reduced in the midmyocardial cells, partly explaining their longest APD across the myocardium ${ }^{134}$. $\mathrm{I}_{\mathrm{Ks}}$ is the major repolarizing current that ends the plateau phase, before $\mathrm{I}_{\mathrm{K} 1}$ conductance takes over and initiates the final repolarization (mid phase 3), restoring the resting membrane potential (phase 4).

vi Refer to section 1.4.3

vii Refer to section 1.1.5 
$\circ$ Final repolarization (phase 3 ) and restoration of $V_{\text {rmp }}$ (phase 4). As repolarization proceeds, $\mathrm{I}_{\mathrm{K} 1}$ channels, which were closed during phase 2 , are progressively reactivated, peaking at the late rapid repolarization phase (mid phase 3) and regenerating $\mathrm{V}_{\mathrm{rmp}}{ }^{136}$. Modulation of $\mathrm{I}_{\mathrm{K} 1}$ is likely to play a profound effect on cardiac excitability and arrhythmogenesis, especially after its role has been highlighted in Andersen-Tawil syndrome ${ }^{137}$, a disease characterized by episodes of muscle weakness, dysmorphic features and a prolonged QT interval on resting ECG. The strong inward rectification of $\mathrm{I}_{\mathrm{K} 1}$ limits the outward currents during phases 0,1 and 2, therefore preventing a short-circuit of the AP and excessive loss of $\mathrm{K}^{+}$into the confined extracellular milieu ${ }^{84}$. The outward I/V relationship of ventricular $\mathrm{I}_{\mathrm{K} 1}$ is defined by an n-shape curve with a negative conductance slope at depolarized potentials ${ }^{90}$. The different $\mathrm{I}_{\mathrm{K} 1}$ channel isoforms $\left(\mathrm{K}_{\mathrm{ir}} 2 . \mathrm{x}\right)$ in the heart have different rectification properties ${ }^{138}$, non-homologous responses to changes in $\left[\mathrm{K}^{+}\right]_{0}{ }^{139}$ and with an interesting heterogeneous distribution throughout the heart ${ }^{140}: \mathrm{I}_{\mathrm{K} 1}$ channels are almost non-existent in nodal cells, whereas the $I_{\mathrm{K} 1}$ current density is 6-10 higher in the ventricles compared to the atria ${ }^{31}$, with a predominance of a stronger rectification behavior in the RV vs. LV141. Such a high density of $\mathrm{K}_{\mathrm{ir}} 2 . \mathrm{x}$ in the ventricles results in a faster repolarization phase and a more hyperpolarized $V_{\text {rmp. The latter has important physiological }}$ consequences. First, it protects ventricular cells from pacemaker activity: SAN cells, which lack $\mathrm{K}_{\mathrm{ir}} 2 \mathrm{x}$ channels, have a relatively depolarized $\mathrm{V}_{\mathrm{rmp}}(\sim-50 \mathrm{mV}$ in SAN vs. $\sim-85 \mathrm{mV}$ in ventricular cells), where small inward nonselective currents, known as the $\mathrm{I}_{\mathrm{f}}$ current 142,143 , are capable of slowly depolarizing the membrane to $V_{\text {thres }}$ during diastole, maintaining automaticity ${ }^{140}$. Second, it confers energetic efficiency in AP generation, by stabilizing $V_{\text {rmp }}$ and cellular input resistanceviii, preventing therefore depressed fast depolarization under normal conditions ${ }^{144}$.

Mechanism of automaticity. Pacemaker activity initiates and sustains electrical excitation in the heart, independently of any central nervous system interference ${ }^{145}$. The intrinsic rhythmicity of SAN cells is determined by an unstable diastolic membrane potential, which merges smoothly with the depolarization upstroke. Phase 4 depolarization is thought to be generated by time- and voltagedependent currents (except for $\mathrm{I}_{\mathrm{Na}, \mathrm{b}}$ ), where the net current must be inward: $\mathrm{I}_{\mathrm{K}}$, delayed rectifier Kcurrent activated during the preceding AP; $\mathrm{I}_{\mathrm{Na}, \mathrm{b}}$, a background current carried predominantly by $\mathrm{Na}^{+}$; $I_{f}$, a non-selective hyperpolarization-activated current; $I_{N C X}$, mediated by the NCX pump; $I_{C a}$, mediated by T- and L-type Ca-channels ${ }^{146}$. The current dogma of automaticity suggests that the basis of automaticity is primarily due to $\mathrm{I}_{\mathrm{f}}$, which is mainly activated at $\mathrm{V}_{\mathrm{mbr}}$ negative to $-40 \mathrm{mV}$. In the preceding $\mathrm{AP}, \mathrm{I}_{\mathrm{K}}$ is outward and contributes to repolarization, followed by $\mathrm{K}_{\mathrm{V}}$ channels closure and decrease in K-conductance. If carries an inward current $\left(\mathrm{Na}^{+}\right.$and $\left.\mathrm{K}^{+} ; \mathrm{V}_{\mathrm{Eq}} \sim-10 \mathrm{mV}\right)$ throughout the range of pacemaker potential, and in the face of decreased K-conductance, it accounts for bringing $\mathrm{V}_{\mathrm{mbr}}$ to threshold. $\mathrm{I}_{\mathrm{Ca}}$ accelerates the rate of diastolic depolarization, generating the upstroke. Most importantly, SAN cells are under parasympathetic control in terms of basal rate, slowing the heart rate to $60-70 \mathrm{bpm}$ in humans. $\mathrm{Ca}^{2+}$ seemed to be involved with pacemaker activity in other ways. In 2010, Lakatta et al. delineated in their landmark report the possibility of a coupledclocks pacemaker system, involving $\mathrm{Ca}^{2+}$ homeostasis and local $\mathrm{Ca}^{2+}$ signaling in the SAN cells rhythmicity regulation ${ }^{147}$. In their presented scheme, the membrane clock ( $M$-clock) that involves the above-described currents remains an integral part of the automaticity paradigm. However, they integrated the rhythmic ryanodine receptor (RyR) -generated subsarcolemmal local calcium releases (LCR) as a $C a$-clock, which appears to be directly coupled to the $M$-clock by regulating $\left[\mathrm{Ca}^{2+}\right]_{\mathrm{i}}$. The details of this regulation are still to be elucidated, but the notion that $\mathrm{Ca}^{2+}$ sequestration and release of $\mathrm{Ca}^{2+}$ from internal stores in SAN cells (similar to the effect seen in ventricular cells)

viii Cellular input resistance is defined as the membrane resistance in the subthreshold voltage range. 
regulates rhythmic cardiac excitation is believed to be an important advancement and a possible shift of paradigms in this area ${ }^{148}$.

\subsubsection{Excitation-Contraction Coupling (ECC)}

The complex process of converting electrical signal to a mechanical contraction is termed ECC. It's closely coupled to a high-energy turnover, offered by commensurate oxygen and nutrients supply to the myocardium for an efficient functioning of its metabolic machinery (oxygen consumption $\sim 0.1 \mathrm{~mL} / \mathrm{g}$.min at basal rates ${ }^{149}$ ). The myocardium is an oxidative muscle (with the highest volume density of mitochondria ${ }^{150}$ in the body and estimated to be $\sim 25.3 \%$ in humans ${ }^{151}$ ), expected to perform for a whole lifetime without fatigue. For that, it's endowed with a rich capillary supply, about one capillary per fiber. Bidirectionally along the length of the fiber, electron micrographs show deep invaginations of the sarcolemma into the fiber, known as transverse or T-tubules ( 200$300 \mathrm{~nm}$ in diameter ${ }^{152,153}$ ). The lumina of T-tubules are continuous with the bulk interstitial fluid, and they play a role key in ECC (Figure $2 \mathrm{~b}$ and Figure $3 \mathrm{~b}$ ).

Striated muscles (skeletal and cardiac) have important morphological and functional differences. However their basic functional unit- the sarcomere, their contractile elements and the slidingcontraction mechanism are quite similar. A striking observation, when comparing cardiac and skeletal tissues with a light-microscope, is the semblance of a syncytium of cardiac muscle with branching interconnecting fibers ${ }^{21}$, with the unique organization of myocyte-myocyte contacts. Even though the myocardium is far from being a true syncytium anatomically, the cardiac muscle behaves as one, when an all-or-none depolarizing wave that is fast enough to cover the whole myocardium in $20 \mathrm{~ms}^{93}$, is followed by a contraction of almost the entire myocardium. Skeletal and cardiac muscles show similar length-force relationship. The developed force is maximal when the muscle begins its contractions at resting sarcomere length $(2-2.4 \mu \mathrm{m})$, where there's optimal overlap of thick and thin filaments with maximal number of cross-bridges 154,155 . In general, the length-force relationship can be extrapolated from the fiber to the level of the entire organ by substituting ventricular systolic pressure for force and end-diastolic ventricular volume for sarcomere length. In the normal heart, the peak force might be attained at a filling pressure of $12 \mathrm{mmHg}$, which corresponds to a sarcomere length of $2.2 \mu \mathrm{m}^{21}$.

Functional unit of contraction ${ }^{2}$. Sarcomeres span the area between Z-lines $(\sim 0.1 \mu \mathrm{m})$. They are constituted of interdigitating thick $(\sim 15 \mathrm{~nm}$ in diameter $)$ and thin $(\sim 10 \mathrm{~nm})$ filaments, which are polymers of noncovenlently associated myosin and actin molecules, respectively (Figure 3a). They are responsible for cardiac muscles' striated appearance (Figure 3d) due to alternating A- (for anisotropic) and I- (for isotropic) bands. Specific proteins stabilize the position of the thick and thin filaments in the sarcomere (such as titin) and others regulate their interactions (such as troponin and tropomyosin). The A-band $(\sim 1.6 \mu \mathrm{m})$ is located nearly in the middle of the sarcomere and is further dissected into 3 regions: the $\mathrm{H}$-band, a less dense zone within the A-band; the pseudo $\mathrm{H}$ band, a lighter zone within the H-band and the M-line, which contains myomesin and it's where thick filaments interconnect in a narrow band of high-density in the pseudo H-band. Thin myofilaments run from their initial attachments on the Z-line, through half the I band $(\sim 1.0 \mu \mathrm{m})$, into the A-band where they overlap partially with the thick filaments. The H-band appears lighter within the A-band, because it doesn't contain overlapping myofilaments (Figure 3b). Titin, known also as connectin, is found in both M- and Z-lines. The integrity of the sarcomere, when the actin and myosin filaments are stretched beyond their overlapping zone, is held together by this giant protein $(\sim 3 \mathrm{MDa})^{156}$, which not only provides a template of the organization of the myofibrillar proteins during development, but also acts as a molecular spring that is responsible for the key aspect of the contractile behavior of the muscle ${ }^{157}$. Titin in the heart muscle has some unique features. 
Figure 3. Molecular basis of cardiac muscle striation and functional domains within the cardiac myocyte. a. An electron micrograph $^{2}$ of longitudinally sectioned myofibrils, showing a sarcomere spanning the area between the Z-lines. A- and I-bands and M-lines are shown (see text). b. Scheme of the cardiac sarcomere in relation to the membranous structures responsible for intracellular $\mathrm{Ca}^{2+}$ handling6. TT: $\mathrm{t}-$ tubule; TC: terminal cisternae; DHPR: dihydropyridine receptor at the TT membrane; RyR: ryanodine receptor $\left(\mathrm{Ca}^{2}+\right.$ release channel of the SR) with foot structures at the TC membrane ${ }^{9}$. M-line; Z-line ${ }^{10}$. MyPB$C$, a hypothetical localization of the myosin binding protein $\mathrm{C}$, localizes to the eleven transverse stripes on either side of the M-line and binds to myosin and titin. Two nebulette molecules are associated with each actin filament, which originate from the Z-line, and tropomodulin caps the actin filaments at their pointed end ${ }^{12}$. c. Schematic representation of the three well-identified specialized domains for channel expression in cardiac myocyte14: (1) the ECC domain composed of ttubule and terminal cisternae of the $\mathrm{SR},(2)$ the costamere at the lateral membrane (see text), and (3) the intercalated disk (ID). d. Laser scanning confocal micrograph of healthy cardiac ventricular tissue stained for F-actin16. Staining shows clear striations. Large, intensely staining bands are most likely to be the ID $(\sim 30 \mu \mathrm{m}$, at white arrow upward) that joins longitudinally adjacent cells (white arrow). Yellow arrows point at the lateral membrane. Pictures taken from references as noted.

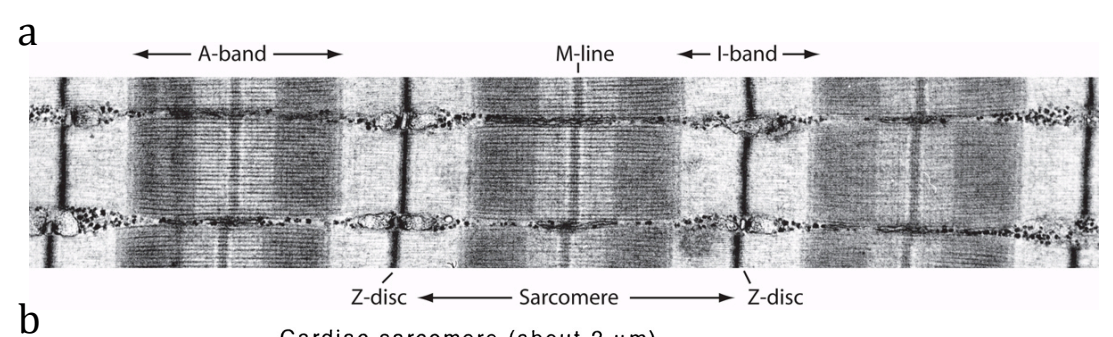

$b$

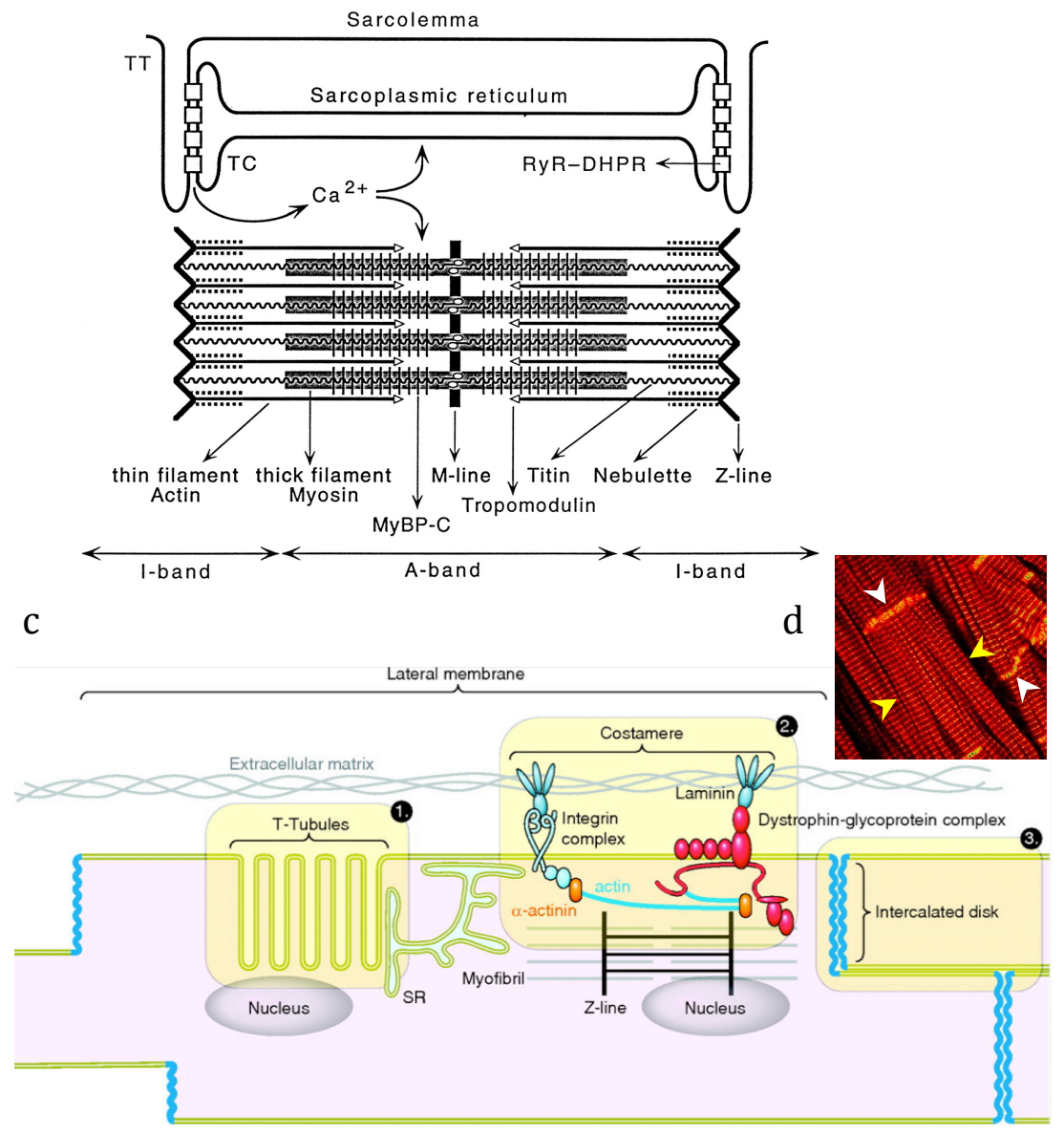

It was suggested that the smaller titin isoform, such as the one seen in the cardiac muscular tissue, sets "the slack length on the ascending part of the length-tension curve"158, which should help the heart adapt to increased filling by stronger contractions (i.e. a better adaptation to higher resting tensions using the Frank-Startling mechanism) ${ }^{53}$. The non-linearity elasticity of titin would not impair filling over a limited range but could provide enough diastolic tone to resist cardiac overfilling 158. Changes in titin isoform expression were found to significantly impact diastolic filling by lowering myocardial stiffness, in end-stage heart failure patients with non-ischemic dilated cardiomyopathy ${ }^{159}$. 
The ECC process is generated at the level of the Z-line, where the t-tubular system allows the functional coupling of electrical activity with the release of $\mathrm{Ca}^{2+}$ from the SR, triggering contraction ${ }^{2}$. In the cardiac myocyte, three crucial structural and functional entities can be delineated: (1) the ECC domain composed of t-tubular system and terminal cisternae of the SR and sarcomere, (2) the intercalated disk (ID) at the cell-cell junction, and (3) the lateral membrane domain ${ }^{14}$ (Figure 3c).

ECC is mediated mainly by $\mathrm{Ca}^{2+}$. A couplon ${ }^{160,161}$ or a local $\mathrm{Ca}^{2+}$ signaling complex is a nanospace within the ECC domain, defined by the cleft between the sacolemma and the SR, where 10-25 L-type channels and 100-200 ryanodine receptors (RyR, see below) are clustered ${ }^{162}$. The t-tubules functionally couple the rise of $\left[\mathrm{Ca}^{2+}\right]_{i}$ from the extracellular to the intracellular domains directly at the Z-line ${ }^{153}$. Upon activation of $\mathrm{I}_{\mathrm{Ca}, \mathrm{L}}$ in the designated couplon, $\left[\mathrm{Ca}^{2+}\right]_{\mathrm{i}}$ rises in $<1 \mathrm{~ms}$ two orders of magnitude from its basal concentration of $\sim 10^{-2} \mu \mathrm{M}$. This local increase in $\mathrm{Ca}^{2+}$ triggers the opening of 6-20 RyR 113 and more calcium is released into the cytoplasm from the SR storage. This process, termed calcium-induced-calcium release or CICR, rises junctional $\left[\mathrm{Ca}^{2+}\right]_{\mathrm{i}}$ to $200-400 \mu \mathrm{M}^{162}$. $\mathrm{Ca}^{2+}$ then diffuses from the cleft to the cytosol to activate the contractile myofilaments. Under normal conditions, $\mathrm{Ca}^{2+}$ doesn't activate the RyRs of the neighboring cleft, which is $\sim 1.5 \mu \mathrm{m}$ away (nearest neighbor: $0.78 \pm 0.07 \mu \mathrm{m}$ ) ${ }^{163}$. This independent function of couplons dictates that all 20,000 couplons in the cardiomyocyte must be simultaneously activated, for a synchronous activation to take place, the dynamics of which normally occurs with each AP164.

Mechanism of myofibrillar activation ${ }^{21}$. The sudden increase in $\left[\mathrm{Ca}^{2+}\right]_{\mathrm{i}}$ due to $\mathrm{I}_{\mathrm{Ca}, \mathrm{L}}$ and $\mathrm{CICR}$ is buffered almost instantaneously by troponin C ( TnC) ${ }^{165}$. The latter along with troponin $\mathrm{T}(\mathrm{TnT})$, troponin I (TnI) and tropomyosin (TM) forms a regulatory unit that controls actin molecules 165 . In the absence of the Ca-TnC complex, TM and TnI hinder the actin-binding site for myosin in a blocked state. The reversible binding of $\mathrm{Ca}^{2+}$ to $\mathrm{TnC}$ results in increased affinity of $\mathrm{TnC}$ to $\mathrm{TnI}$, which releases its inhibitory control on actin. TM, partially released from TnT, performs initially a $\sim 10^{\circ}$ rotation and partially exposing the actin binding site; therefore promoting myosin hydrophobic interactions, which will further rotate TM an additional $25^{\circ 166}$. At this point, all actin binding sites are available for interaction with the myosin heads ${ }^{167}$. This contributes to the strong cooperativity observed in myofilament sensitivity curves ${ }^{168}$. When the binding takes place, actin activates the ATPase, and splits an ATP molecule on the myosin head. The energy released decreases the angle of contact between actin and myosin from $90^{\circ}$ to $45^{\circ}$, thus pulling the actin filament. Detachment of myosin from actin requires another ATP binding to myosin ${ }^{166}$. As long as $\left[\mathrm{Ca}^{2+}\right]_{i}$ is high enough for sustaining the formation of the Ca-TnC complex, a new cycle of crossbridges begins. Many such cycles occur in single muscle activation, mainly due to the reciprocal Ca-force interaction. Crossbridges' binding and force generation enhance the affinity of $\mathrm{Ca}^{2+}$ binding to $\mathrm{TnC}$, thereby prolonging the active state of contraction ${ }^{162}$. As $\mathrm{Ca}^{2+}$ is being sequestered back into the $\mathrm{SR}$ via active transport, the decrease in $\left[\mathrm{Ca}^{2+}\right]_{\mathrm{i}}$ breaks the Ca-TnC complex, reestablishing resting conditions ${ }^{74}$.

$\mathrm{Ca}^{2+}$ reuptake via SERCA. The active cytosolic $\mathrm{Ca}^{2+}$ transport into the SR is accomplished by SERCA pumps, which exist in 3 different isoforms ${ }^{169}$. SERCA2 is the cardiac isoform. Both protein kinase $A$ (PKA) and Ca-Calmodulin protein kinase (CaMKII) can regulate $\mathrm{SR} \mathrm{Ca}^{2+}$ reuptake via phospholamban (PLB) phosphorylation, removing the continuous SERCA2 inhibition by PLB170. $\mathrm{Ca}^{2+}$ is also extruded out of the cell through the electrogenic pump NCX ${ }^{i x}$. In the steady state, a delicate $\mathrm{Ca}^{2+}$ balance is maintained, where the amount of $\mathrm{Ca}^{2+}$ taken up by the SR during relaxation must equal the amount released and the amount that enters through $\mathrm{I}_{\mathrm{Ca}, \mathrm{L}}$ must equal the amount extruded by $\mathrm{NCX}^{162}$. The fraction of activating $\mathrm{Ca}^{2+}$ that returns back into the SR and out to the cell varies among species 113 . In human ventricles, almost $70 \%$ of activating $\mathrm{Ca}^{2+}$ are cycled through the SR, the remaining $20-28 \%$

ix Refer to section 1.1.4 
through the $\mathrm{NCX}^{113}$. In contrast, more than $95 \%$ is cycled through the SR in mouse and rat ventricle ${ }^{113}$. In heart failure (HF), in which typically SERCA function is decreased and NCX function is enhanced, this balance shifts to almost $50-50 \% 171$.

The SR Ca ${ }^{2+}$ release channel or ryanodine receptor (RyR). The diversity of SR release properties is not yet completely understood. RyR is among the largest known proteins, consisting of homotetramers each over 5,000 amino acids with a molecular mass of $\sim 550 \mathrm{kDa}^{172}$. RyR is present in mammalian muscles in distinct isoforms: RyR1 is the dominant one in skeletal muscles and RyR3 is restricted to a few skeletal muscles in the post-natal period ${ }^{173}$. RyR2 is the cardiac isoform and is never expressed in skeletal muscles ${ }^{172,}$ 174. Since the expression of specific RyR2 isoform isn't diverse in the cardiac muscle, modulation of RyR2 activity is related to many factors including many interacting proteins.

- Calsequestrin (CASQ) is the most important binding protein inside the SR and is mainly located in the terminal cysternae in close proximity to RyR. Due to its acidic residues, the multimeric CASQ2 (cardiac isoform) can bind up to $50-75 \%$ of the $\mathrm{Ca}^{2+}$ taken up by the $\mathrm{SR}^{113}$, 175. Extensive research is ongoing to determine CASQ modulation of RyR, where mutations in CASQ were shown to trigger exercise-induced ventricular arrhythmia (Catecholaminergic Polymorphic Ventricular Tachycardia or CPVT) ${ }^{176}$. Although direct effects of CASQ2 on RyR2 have been described, it's now believed that CASQ2 modulates RyR2 function through integral membrane proteins triadin (TRD) and junctin (JN) ${ }^{177}$. When $\left[\mathrm{Ca}^{2+}\right]_{\mathrm{SR}}$ is low, CASQ2 is bound to TRD and/or JN, stabilizing RyR2 closure. When $\left[\mathrm{Ca}^{2+}\right]_{S R}$ is restored, CASQ2 unbinds from TRD/JN and the inhibitory effect of CASQ2 on RyR2 terminates, increasing opening probabilities of RyR2175, 178 .

- DHPR. The coupling between those two proteins differs tremendously between cardiac and skeletal muscles ${ }^{179}$. While $\mathrm{Ca}^{2+}$ is indispensable for the CICR in cardiac muscles, which is accelerated by the vicinity of DHPR to RyR2; the direct mechanical coupling between DHPR and RyR that prevails in the skeletal muscle is $\mathrm{Ca}^{2+}$ independent and DHPR's role is reduced to the one of a voltage sensor for RyR opening ${ }^{180}$. An index of the proportion of RyR directly controlled by the DHPR is given by the ratio DHPR:RyR. This index was shown to decrease with aging in skeletal muscles ${ }^{181}$. However it's not known yet, whether this process occurs in the heart as well.

- Calmodulin or CaM. This molecule ubiquiously binds cytosolic $\mathrm{Ca}^{2+}$ and affects RyR2 function by direct binding ${ }^{182}$. At $\left[\mathrm{Ca}^{2+}\right]_{\mathrm{i}}<1 \mu \mathrm{M}, \mathrm{CaM}$ inhibits opening of the RyR2183. Ca-dependent inactivation of $\mathrm{I}_{\mathrm{Ca}, \mathrm{L}}$ is also mediated by CaM: upon $\left[\mathrm{Ca}^{2+}\right]_{\mathrm{i}}$ increase, the Ca-CaM complex binds to the IQ motif of DHPR, accelerating $\mathrm{I}_{\mathrm{Ca}, \mathrm{L}}$ inactivation ${ }^{184}$.

- FKBP-12 and FKPB-12.6 are both classified as immunophilins and modulate $\mathrm{Ca}^{2+}$ release properties of RyR2185; in particular, they stabilize the full conductance or the closed conformation state of the channel186. PKA phosphorylation of RyR2 dissociates FKBP-12.6 and regulates the channel open probability ${ }^{187}$.

\subsection{Anisotropy}

Lewis et al. recorded the first observation pointing to the link between CV and the direction of propagation in the cardiac muscle, in 1914188. The propagation of excitation in the cardiac muscle has generally been approached as though it occurred in a continuous excitable medium - the Hodgkin-Huxley equations of excitation and propagation were modeled on the giant squid axon ${ }^{86}$ for instance. In fact, the cellular and tissue structures in the heart are a confluence of discontinuities brought together through highly organized specialized electrical and mechanical coupling systems ${ }^{189}$. The result is this distinctive spatial distribution of propagation, termed anisotropy, where the impulse propagation is faster in the direction parallel to the cardiac fiber, than in the 
direction perpendicular to it 21,190 . Anisotropy is the consequence of characteristics detected at the level of the cell, as well as the multicellular tissue. The former is attributed to spatial nonuniformities in the membrane properties, whereas the latter to the specialized sarcolemmal regions containing arrays of densely packed transmembrane channels connecting the cytoplasmic compartments of adjacent cells ${ }^{100}$. As introduced in the beginning of section 1.1.5, the cardiac cell is a highly differentiated structurally polarized cell with well-developed domains, where the ECC domain was described. In this section both the ID domain, and the remaining lateral membrane (LM) domain are described.

\subsubsection{Passive Molecular Determinants of Anisotropy}

By examining a healthy cardiac tissue sectioned in a plane parallel to the direction of its fibers, (Figure 3d), highly dense structures appear to connect two adjacent cardiomyocytes. These intercalated disks (ID) contain multiple adhesive components that ensure mechanical coupling and rapid coordinated electrical propagation, therefore are vital for the integrity of the myocardium and its ability to function as a whole ${ }^{191}$. Three types of adhesive junctions exist in the ID domain: fascia adherens and desmosomes, which are primarily mechanical; whereas gap junctions are the sites of intercellular electrical coupling.

Fascia adherens and desmosomes anchor actin filaments (described previously, involved in the contractile machinery) and intermediate filaments (a component of the cytoskeleton) at the plasma membrane of adjoining cells respectively, thereby provide physical and mechanical attachment between the cells ${ }^{191,192}$. In fascia adherens, transmembrane protein $\mathrm{N}$-cadherin connects sarcomeric actin filaments and maintains strong cell-cell adhesion. Desmosomes are small and discrete structures composed of three separate families of proteins: desmosomal cadherin, armadillo proteins and plakins ${ }^{193}$. In the normally working myocardium, both fascia adherens and desmosomes are involved in the sensing and regulation of mechanical stresses acting along the longitudinal axis. The area composita, a mixed type of junctional structure, is exclusive to the ID of mammalian species suggesting an evolutionary mechanism to strengthen mechanical coupling in the heart of higher vertebrates ${ }^{194}$. Their role is essential in organs subjected to high mechanical stresses, such as the skin and the heart. Mutations in genes coding for these junctional proteins are involved in cardio-cutaneous inherited pathologies ${ }^{195}$, such as Naxo's disease ${ }^{196,197 .}$

Connexin43 ( $\mathrm{Cx} 43)$ is the major protein constituting gap junctions, which are made of two connexons (or hemichannels) ${ }^{198}$ and allow non-selective diffusion of $\sim 1 \mathrm{kDa}$ molecules ${ }^{199}$, ions, and second messengers, thereby offering electro-metabolic coupling. 3 types of connexins exist in the heart: $\mathrm{Cx} 40,43$ and 45 (the number refers to their molecular weights; 43 being the most abundant). Saffitz et al. describe the spatial distribution of gap junctions as well suited for the heart's functional requirements 200 , where large gap junctions at the ends of cells presumably facilitate the efficient intercellular current transfer and provide favorable current-to-load conditions ${ }^{201}$. Gap junction specific proteins and density have a direct influence on the velocity of propagation. The measured $\mathrm{CV}$ in Purkinje fibers $\left(\sim 3 \mathrm{~m} . \mathrm{s}^{-1}\right)$ is considerably higher than the one recorded in the ventricular bulk

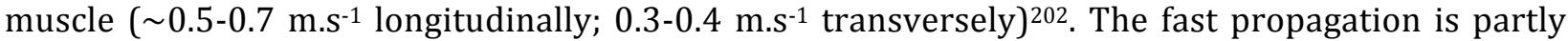
due to the presence of different connexins in the gap junctions of these cells, where the amount of $\mathrm{Cx} 40$ is at least 3 folds higher in Purkinje fibers than regular cardiomyocytes ${ }^{203}$. On the other hand, high anisotropic ratio (AR) was also attributed to the shape of cells, the number and spatial orientation of cellular connections rather than the anisotropic distribution of gap junctions ${ }^{190}$. The

x Saffitz et al. defined current-to-load as the difference between the minimum amounts of current required in maintaining conduction and the actual amount delivered. 
crista terminalis, a discrete bundle of atrial fibers that conducts impulses from the SAN to the AVN, is characterized by a relatively high AR compared to the bulk ventricular muscle $(\sim 10$ vs. $\sim 3$ respectively), despite the relatively fewer gap junctions present ${ }^{201}$. It was suggested that because of the elongated shape of the cells found in the crista terminalis, wave fronts traveling in the transverse direction must traverse more intercellular junctions per unit distance traveled, and thus would encounter more resistance to their propagation than wave fronts traveling in the longitudinal direction ${ }^{190,204 .}$

In reality, mechanical stresses can induce changes in gap junction density at the ID, subsequently affecting the intercellular communication and propagation velocity on the macroscopic level. Saffitz et al. argue that the close proximity of gap junctions to mechanical adhesive junctions within the ID, and their ribbon like structures oriented perpendicular to the long axis of the cell is a revolutionary adaptation that would protect them from shear stress ${ }^{200}$. In favor of this point, pulsatile mechanical stretch was shown to markedly upregulate $\mathrm{Cx} 43$ in cultured neonatal cardiomyocytes, inducing remodeling and redistribution of gap junctions and possibly alterations in electrical conduction ${ }^{205}$. In addition, observations suggest that mechanical integrity is a requirement for a normal excitation, and formation of adhesion junctions is a prerequisite for gap junction formation ${ }^{206}$. On the other hand, $\mathrm{Cx} 43$ was not found to be a prerequisite for the organization of mechanical junctions ${ }^{192}$ and that electrical conduction was maintained in $\mathrm{Cx} 43$-deficient mice, suggesting an alternative mechanism of electrical propagation in the context of severely reduced gap junctions ${ }^{207}$. A growing line of evidence suggests that altered mechanical coupling or loss of cell-cell contact for any reason (chemical mediator, hypertrophy, dilatation, scarring... etc.) would directly modulate electrical pacing of the heart. Whereas, the opposite is not yet as tangible: impaired electrical coupling doesn't affect mechanical coupling 206 .

Under normal physiological conditions, cardiac cells don't show similar electrical coupling along the transverse axis, as they do along the longitudinal one ${ }^{14}$. The costamere and the t-tubules are the two major structures in the LM domain (Figure 3c). The term costamere refers to the rib-like bands that encircle the cardiomycoyte perpendicular to its long axis ${ }^{208}$. It consists of a complex protein network that not only forms a physical attachment of the underlying Z-line to its outer stress tolerant extracellular matrix (ECM), but also is the site where contractile forces within the cardiomyocyte are directly transmitted to the ECM ${ }^{209}$. In fact, externally applied strain on the ECM was sufficient through this protein complex to induce myofibril contraction, indicating that both externally applied and intrinsically stimulated mechanical forces were bidirectionally transmitted through the costameres ${ }^{209}$. The major consequence of muscular contraction is shortening and deformation. A process during which the contractile machinery of the sarcomere must remain connected to both the sarcolemma and the ECM, for the adequate transmission of force and the proper coordination of contraction within the three-dimensional muscular structure ${ }^{210}$. Cardiomyocytes, as load-bearing cells, not only generate and transmit mechanical contractions, but they are also equipped through the costameres to sense physical forces, to transduce them into biochemical signals, and generate appropriate responses leading to alterations in cellular structure and function ${ }^{211}$. This mechanotransduction is particularly complex in the heart, since individual cardiac cells should adapt to many simultaneously ongoing processes 212 : externally applied mechanical forces, internal loads (blood volume, arterial pressure), neurohormonal modulations, by either reacting instantaneously on the short-term or modifying gene expression for long-term responses ${ }^{212}$, then transmitting the same message to adjacent cells and their surrounding ECM for a synchronized contraction.

Two important macromolecular protein complexes play the regulatory role at the costamere: the dystrophin-syntrophin mutliprotein complex (DSMC) and the integrin complex ${ }^{14}$ (Figure 3c). Integrins 
are heterodimers composed of chains with a long extracellular domain, which binds extracellular laminin to the cytoskeletal actin ${ }^{213}$. The principal role of the integrin complex is mechanotransduction, anchoring at its cytoplasmic side many signaling molecules and kinases. Inactivation of the cardiac integrin gene in mice resulted in an enhanced predisposition to stressinduced cardiomyopathy ${ }^{214}$ and dilated ventricular chambers ${ }^{215}$.

\subsubsection{The Dystrophin Molecule and the DSMC}

The DSMC, similarly to the integrin complex, is composed of transmembrane, cytoplasmic and extracellular proteins ${ }^{216}$. In the cardiac cell, the transmembrane proteins are the sarcoglycans, the sarcospan and the dystroglycans that in turn bind to extracellular laminin. On the cytoplasmic side, dystrophin binds to syntrophin, dystrobrevin ${ }^{217}$, and neuronal nitric oxide synthase and attaches this multimolecular complex to cytoskeletal actin ${ }^{218}$. The DSMC is essential in stabilizing the sarcolemma upon physical stresses. Dystrophin $(\sim 430 \mathrm{kDa})$ is mainly expressed in striated muscles, including cardiac muscle cells ${ }^{219}$ and its main function lies in linking membrane proteins to the actin cytoskeleton $^{220}$ and maintaining the stability of the dystrophin-syntrophin multiprotein complex (DSMC) in the LM domain ${ }^{221}$. An interesting fact is that dystrophin isn't restricted to muscles, but is also expressed in the central nervous system (such as the cerebellum, cortical neurons, hippocampus) ${ }^{222}$. Mutations of dystrophin lead to a severe progressive muscle wasting disease, Duchenne muscular dystrophy (DMD), or to a less severe form Becker's dystrophy ${ }^{223}$.

DMD is a devastating X-linked degenerative muscle disease, with an incidence of 1:3300 live male births annually 223 . In patients with DMD, muscular biopsies characteristically demonstrate encoring or degenerating muscle fibers, often observed in clusters ${ }^{224}$. Small immature centrally nucleated cells are also observed and they correspond to the regenerative processes that take place in the early phases of the disease, creating a balance between necrotic and regenerating muscle tissue ${ }^{225}$. Later, the regenerative capacity of the muscle is exhausted and muscle fibers are continuously replaced with connective and adipose tissues ${ }^{225}$. The pathology becomes more prominently manifested with age and patients are usually wheelchair ridden in early adolescence ${ }^{224}$.

The responsible gene and its product, dystrophin, have been identified more than 30 years ago ${ }^{226}$, and the mouse model $(m d x)$ harboring a spontaneous mutation similar to the one observed in man has been extensively studied, as a mouse model of the pathology 227,228 . These experimental murine models are one of the best tools not only to investigate the primary defect, but also to delineate the causal chain of events leading to the observed pathological finding229, 230. In the case of DMD, despite major advancements done in the field, a comprehensive understanding of the mechanism leading from the absence of dystrophin to the muscular degeneration is still lacking231. Several hypotheses have been proposed to clarify the pathophysiology of the underlying deficits resulting from the absence of dystrophin ${ }^{232}$. Among the current hypotheses is the mechanical hypothesis, which suggests that membrane fragility in DMD patients allows the accumulation of proteins that are not normally present in the muscle fibers, indicating increased membrane permeability ${ }^{233}$. On the other hand, the calcium hypothesis proposes that disruptions to the DSMC can result in instability of the sarcolemma that permits calcium entry through membrane tears, when the sarcolemma is stretched during lengthening muscle contractions ${ }^{234}$. For a comprehensive review on the different available hypotheses involved in the pathology of DMD, refer to the review by Deconinck and Dan (2007)231. 
The importance of dystrophin from a cardiac perspective becomes obvious when almost all DMDpatients ${ }^{\mathrm{xi}}$ develop severe cardiac manifestations of their disease, that culminate into dilated cardiomyopathy ${ }^{235}$, heart failure and increased propensity of sudden cardiac death ${ }^{224}$. Henceforth, understanding the mechanisms behind the induced arrhythmia in the dystrophic substrate requires the knowledge not only of the membrane effects due to the primary channel defect, but also of the intracellular signals, developmental effects on intercellular communication, integration of possible compensatory responses and other environmental factors that modulate the translation of the primary defect from the gene to the organ ${ }^{229}$. Despite known discrepancies in the electrophysiology of mouse and man, the $m d x$ mouse has been continuously developed in the last 30 years to elucidate the role of dystrophic loss in the pathology of DMD.

Dystrophin has multiple protein-protein interaction domains, including a specific domain in its carboxylic terminus facilitating its interaction with syntrophin ${ }^{217}$. The latter has a PDZ domain xiithat mediates interactions with the carboxylic end of various ionic channels, including $\mathrm{Nav} 1.5^{\text {xiii, which }}$ has a PDZ-domain binding motif in its carboxylic-terminal tail. Interestingly, among the nine different $\mathrm{Na}_{v}$ channels, only the ones expressed in striated muscles (i.e. Nav1.4 and $\mathrm{Na}_{v} 1.5$ ) have such PDZ-binding motifs in their carboxylic ends ${ }^{236}$. This domain was found necessary for indirect interactions with dystrophin via syntrophin ${ }^{236}$. Gavillet et al. have initially shown that $\mathrm{Na}_{v} 1.5$ expression was decreased in the $m d x$ mouse, the functional consequence of which was a reduction in the whole cell $\mathrm{I}_{\mathrm{Na}, \mathrm{f}}$ current ${ }^{237}$. The authors ruled out other factors that could potentially lower the availability of $\mathrm{Na}_{v} 1.5$, hence decrease the upstroke current (such as increased $\mathrm{V}_{\mathrm{rmp}}$, or alterations in activation-inactivation kinetics of $\mathrm{Na}_{v} 1.5$ in the $m d x$ heart). As dystrophin and syntrophin are virtually absent from IDs in normal condition, Petitprez et al. subsequently demonstrated that the interaction between $\mathrm{Na}_{v} 1.5$ and dystrophin is exclusive to the LM of the myocytes ${ }^{238}$. All these findings combined, suggest that $\mathrm{Na}_{\mathrm{v}} 1.5$ is exclusively regulated by dystrophin on the LM of cardiomyocytes, the absence of which in the dystrophin-deficient mouse ensues the loss of Nav1.5 from the LM and reduction in the total $\mathrm{I}_{\mathrm{Na}, \mathrm{f}}$ at the upstroke. On the other hand, other studies have emphasized the interaction of SAP97 (another regulatory protein described in section 1.4.6) with cardiac ionic channels 239,240 including $\mathrm{Na}_{v} 1.5$ at the IDs ${ }^{238}$, which also involves the PDZ-domain. In these studies, SAP97 enhanced $\mathrm{I}_{\mathrm{Na}, \mathrm{f}}$ without affecting the biophysical properties of the current or the intrinsic properties of the channels ${ }^{238}$. SAP97 has been also found not to have any major influence on conductance or opening probability nor time of the channels; rather it is thought to enhance the density of functional channels 241 . Silencing SAP97 lead however to a drastic deterioration of both $\mathrm{I}_{\mathrm{Na}, \mathrm{f}}$ and $\mathrm{I}_{\mathrm{to}, 1}$ (the transient outward $\mathrm{K}^{+}$current or early repolarization current) ${ }^{238}$. How SAP97 modulates functional $\mathrm{Na}_{\mathrm{v}} 1.5$ is not yet well understood.

\subsubsection{Active Determinants of Anisotropy}

$\mathrm{Na}_{\mathrm{v}} 1.5^{\mathrm{xiv}}$ is a transmembrane protein, the localization of which has been subject to controversy. Studies that have investigated the localization of $\mathrm{Na}_{v} 1.5$ have described channel staining within the surface of t-tubular membranes, concentrated principally in the perinexus region near gap junctions ${ }^{242}$ and more exclusively at the intercalated disks (IDs) 243,244 . Initial evidence of the subcellular distribution of $\mathrm{Na}_{v} 1.5$ in adult mammalian cardiomyocytes was provided by Haimovich et al. (1984) with their immunohistochemical staining of muscle cells ${ }^{245}$. Cohen et al. subsequently demonstrated that $\mathrm{Na}_{\mathrm{v}} 1.5$ inhomogeneity had a distinct subcellular pattern in adult rat ventricular

\footnotetext{
xi DMD stands for Duchenne Muscular Dystrophy, a severe progressive muscle wasting disease secondary to mutations in the dystrophin gene, followed by the complete absence of the protein in the striated muscles of affected individuals. xii The PDZ is a protein-protein interaction domain. Refer to section 1.4.6 under "Anchoring and modulating proteins".

xiii The cardiac Na-channel (Nav1.5) is addressed in detail in section 1.4.

xiv The cardiac Na-channel (Nav1.5) is addressed in detail in section 1.4.
} 
muscle, in which there was intense immunolabeling of $\mathrm{Na}_{v} 1.5$ at the IDs with little immunolabeling of the remaining surfaces 242 . In the paper by Petitprez et al. authors demonstrate that $\mathrm{Na}_{\mathrm{v}} 1.5$ is present all along the plasma membrane of both rat and mouse cardiomyocytes ${ }^{238}$. Reduction of lateral $\mathrm{Na}_{v} 1.5$ expression in the dystrophin deficient $(m d x)$ myocytes without measurable alteration in the expression of the channel at IDs proposes the existence of at least two pools of Nav1.5 in the cardiac tissue regulated by a distinct set of proteins. This finding is consistent with previous measurements done on isolated cardiac myocytes showing a 50\% decrease in the total expression of $\mathrm{Na}_{\mathrm{v}} 1.5$ in the absence of dystrophin, with an ancillary $\mathrm{I}_{\mathrm{Na}, \mathrm{f}}$ reduction ${ }^{237}$. These results combined suggest that possibly a considerable fraction of Nav1.5 is located outside the IDs, the loss of which from the LM in the $m d x$ cardiac cell is likely to be associated with conduction abnormalities supported by the previously found significant decrease in $\mathrm{I}_{\mathrm{Na}, \mathrm{f}^{238}}$.

\subsection{Complexity of Activation in the Cardiac Tissue}

The heart, as described before in section1.1.1, has a complicated 3D architecture (Figure 1), in which the 3D arrangement of the different types of cells impinge on our ability to understand how propagation "really" occurs in the heart. Particular emphasis has been given recently to the 3D myocyte composition at the organ scale ${ }^{246}$. Knowledge of 3D propagation depends on our ability to image and resolve 3D structure and to make functional measurements that are relevant to the underlying physiology. It's currently accepted to consider 2D measurements of cardiac tissue as appropriate models to investigate propagation, since images of myocardial tissue with sufficient resolution have visualized cells in 2 dimensional rather than 3 dimensional dimensional structures ${ }^{246}$. At the level of the whole organ, the geometry of the heart can be reconstructed in 3D, using measurements done at tissue scale resolution using various imaging modalities 247-249. Measuring 3D electrical activity in the heart wall with equivalent precision is still considered a challenging task from a technical perspective. Validation of 3D reconstruction using 2D measurements performed on the tissue scale will have to wait until sufficiently high spatialtemporal resolution becomes available for 3D whole-heart measurements 250 .

\subsubsection{Continuous vs. Discontinuous Propagation in Cardiac Muscle}

Propagation of electrical activity in the heart has generally been treated as though it occurred in a homogeneous and continuous medium, such as the squid axon that motivated the pioneering work of Hodgkin and Huxley in 195286. Spach argues that this perspective to the cardiac muscle came about not because it was assumed to be anatomically or histologically "continuous", rather because the continuous cable theory showed an obvious success when implicated in excitable neurons and many early cardiac experiments showed a consistency with the results found in nerves ${ }^{251}$. Indeed there are palpable analogies between a cardiac fiber and a continuous nerve axon, that have been exploited in the modeling of cardiac AP propagation ${ }^{252,253}$. For a propagating AP, the distribution of transmembrane voltage ahead of the depolarization wave front is the outcome of two simultaneous events: the discharge of the depolarized membrane and redistribution of inward currents ${ }^{246}$. Increasing the inward flux of depolarizing charges will raise the rate at which the neighboring quiescent tissue reaches threshold, which will consequently fasten $\mathrm{CV}$ in the medium. A decrease in membrane time constant will translate into a decrease in the corresponding CV ${ }^{254}$. Computer modeling provides an effective framework for integrating experimental data acquired at the cell and molecular levels. Multiscale models that integrate cell activation models within a 3D anatomy offer a means of simulating macroscopic scale cardiac activation. Typically, these finite element models assume electrical properties to be continuous within the cell and across gap junctions ${ }^{255}$. These assumptions will tend to homogenize electrical activity across large tissue volumes, which can reduce the efficiency of these models to detect heterogeneity in activation on a large scale ${ }^{246}$. 
Evidence has shown that propagation in the cardiac muscle rather displays a discontinuous nature: Microscopic components derived from active ionic and passive membrane properties, and macroscopic discontinuities reflecting branching anatomical features or other structural heterogeneities, can produce changes in the shape of the AP and CV, characteristics of cardiac conduction that cannot be predicted by continuous propagation models ${ }^{256}$. Discontinuous propagation implies that the delicate local source-sink balance governs successful conduction, where the amount of charges supplied by the source must be at least equal to the charges required to excite the cardiac membrane at the sink ${ }^{257}$. At the cellular level, this is controlled by membrane excitability followed by the state of electrical coupling between cells. Predictions of discontinuous propagation differ extensively from the ones predicted by continuous models. For instance, gap junctional conduction has been represented by reciprocal values of simple resistors in continuous models of propagation. Double voltage clamp measurements, used to describe the kinetics of single connexins, showed that gap junctional conductance was a dynamical process, during which it increases moderately immediately after the passage of the wave front ${ }^{258}$. Conduction is therefore relatively rapid within cells with long delays across junctions that increase progressively as coupling between cells is reduced. These key features are necessary to be fully captured in more realistic models of cardiac propagation, to improve our understanding of mechanisms based on propagation instabilities in arrhythmogenesis.

In the ventricular myocardium, the principal active determinant of excitability is probably the cardiac $\mathrm{Na}_{v} 1.5^{\mathrm{xv}}$, where the magnitude of the fast-inward $\mathrm{Na}^{+}$current $\left(\mathrm{I}_{\mathrm{Na}, \mathrm{f}}\right)$ plays the decisive role in the subsequent propagation of the electrical wave from the source location further across the tissue. In a simplistic fashion, this axial current flows along the cellular network from one depolarized myocyte to its quiescent neighbor via gap junctions. If this current is of sufficient magnitude, it will depolarize the resting cell beyond its activation threshold, forcing Nav1.5 channels to acquire an open-state conformation at takeoff of the AP upstroke for $\sim 1 \mathrm{~ms}$ and inactivate almost instantaneously ${ }^{259}$. Meticulous opening and closing of the cardiac ion channels results in the generation of the AP, ensures its successful propagation, maintains the intricate coupling of electrical and mechanical activities and orchestrates the sequence of ionic channels to bring about the lucrative termination of the $\mathrm{AP}^{260}$. Henceforth, abnormalities of $\mathrm{Na}_{\mathrm{v}} 1.5$ expression, regulation or kinetics will translate into cardiac instabilities that induce electrical vulnerability and precipitate rhythm disturbances.

\subsubsection{Natural Heterogeneity in the Heart}

The early investigations done on the cardiac tissue were limited to the functional data gathered from either Purkinje fibers or from the ventricular myocardium. Antzelevitch and colleagues pioneered the notion that heterogeneities of cellular repolarization in different cell types can by itself represent a mechanism for the propensity of arrhythmia in pathologies that are associated with sudden cardiac death 261,262 . There exists currently ample evidence that the ventricular myocardium is comprised of 3 functionally different types of cells: epicardial, midmyocardial and endocardial, which exhibit distinct electrophysiological properties and pharmacological profiles ${ }^{263}$, 264. The relative expression of channels mediating repolarizing currents (mainly the $\mathrm{K}_{\mathrm{V}}$ channels) appears to contribute substantially to the electrophysiological heterogeneity of the ventricle, and the observed morphological differences in ventricular AP at different sites of the myocardium ${ }^{103}$. Although differences between action potential characteristics of ventricular subepicardial and subendocardial tissues have long been recognized, systemic studies tackling the

xv The cardiac Na-channel (Nav1.5) is addressed in detail in section 1.4 
electrophysiological differences from a molecular and pharmacological perspective have been slightly delayed 265 , until studies by Antzelevitch and colleagues highlighted the differences in AP morphology, ionic channels and pharmacological responses between the different ventricular layers ${ }^{266}$. They emphasized on the role played by the transient outward current ( $\left.\mathrm{I}_{\mathrm{to}, 1}\right)$ prominently present in the epicardium, but not endocardium in generating the repolarization gradient and the observed difference in rate dependency of the APD (i.e. different restitution properties) across the ventricular wall ${ }^{267}$. The hallmark of midmyocardial cells (or M-cells), which lie between the epicardial and endocardial surfaces along with transitional cells, is the ability of their corresponding APD to exceed epicardial or endocardial APD in response to different factors such as heart rate, or agents that normally prolong the APD ${ }^{268,269}$. As these cells are strongly coupled to one another in the normal myocardium, the repolarization gradients typically found in isolated cells from different layers of the ventricular wall are smoothed out, and together, the degree of electrotonic coupling and intrinsic differences in APD determine the extent of the spatial dispersion of repolarization (SDR) 264, 270. Antzelevitch also postulated that although spatial and temporal heterogeneity is a constitutional property of the ventricular wall, amplification of transmural heterogeneities leading to an SDR accentuation is the principal arrhythmogenic substrate in acquired or congenital SCD syndromes, such as Long QT (LQTS)xvi264.

\subsubsection{Role of Heterogeneity in Cardiac Pathologies}

The role of functional heterogeneity has only started to unwrap in the context of cardiac pathologies, whether acquired ones (such as in heart failure (HF) ${ }^{271}$, ischemia ${ }^{272}$, hypoxia 273 , hyperkalemia $274,275 \ldots$ etc.) or congenital ones (such as in LQTS261, Brugada Syndrome276, Arrhythmogenic Right Ventricular Dysplasia277, Catecholaminergic Polymorphic Ventricular Tachycardia $278 \ldots$ etc.). Experimental studies done on arterially perfused LV canine wedges showed that under conditions where intrinsic heterogeneity was amplified, triggered activity further reduced the net repolarizing currents, making the ventricular wall more prone to develop arrhythmias 279 , 280. Models of LQTS suggest that preferential prolongation of the M cell APD, under conditions such as adrenergic stimulation, leads to an increase in the QT-interval, as well as increase in SDR, which contributes to the development of spontaneous and stimulated arrhythmias 281 . The investigators found these results to be in concordance with what is reported clinically, since sympathetic influences in some subtypes of LQTS patients is known to increase the QT-interval and precipitate ventricular arrhythmias ${ }^{282}$.

Prominence of High Resolution Optical Mapping in Characterizing Heterogeneity. Highly complex, interactive and dynamic changes in the structural, metabolic and electrophysiological properties of the cardiac tissue collectively predispose the heart to lethal electrical disturbances. Despite major advances done on the molecular and cellular levels to characterize cardiac vulnerability to arrhythmias, the effects observed level on the cellular level cannot predict the mechanism involved in facilitating arrhythmias in the multicellular tissue and on the organ level. High-resolution optical mapping is therefore a critical tool to measure and characterize the macroscopic translation of these molecular and ionic channel defects, particularly when the mechanisms of arrhythmogenesis are based on multicellular effects such as SDR.

The importance of using optical mapping measurements is not restricted to the evaluation of temporal gradients in the heart, but is also of crucial importance in describing global effects in conduction. Conduction velocity (CV) has been evaluated with more traditional tools before optical mapping, the results of which have been considered relevant, since it has been assumed that only

xvi See section 1.4.3 for more details on Long QT Syndrome (LQTS). 
two requisites are necessary to measure CV in a medium: a spatial component (distance) over a temporal one (time). These simplistic assumptions, although contributed significantly to our understanding of electrical conduction in the cardiac tissue, will obviously fail when conduction becomes a global outcome of heterogeneously distributed zones. For instance, $\mathrm{Cx} 43^{\mathrm{xvii}}$ depletion has been widely investigated in the context of heart failure (HF), where a reduction of $\sim 50 \%$ of this protein have been reported in end-stage HF in humans ${ }^{283}$. In animal (murine) models, high resolution mapping studies revealed no change in CV with $\mathrm{Cx} 43$ depletion up to $60 \% 284,285$. However $>80 \% \mathrm{Cx} 43$ depletion was necessary to cause a reduction in CV, an altered anisotropy ${ }^{286}$ and an increased susceptibility for ventricular tachycardia 287 . Be that as it may, it has been argued that such a severe global reduction in Cx43 may not be clinically attainable on one hand, for such a molecular mechanism to occur in the failing human heart ${ }^{283}$. On the other hand, it has been shown that cardiomyopathy is rather associated with a spatially heterogeneous distribution of Cx43, which ensures that some regions of the failing heart might probably reach the threshold depletion of $\mathrm{Cx} 43$ at which impulse propagation could be sufficiently slowed, providing therefore a substrate for reentry ${ }^{288}$. In this regard, optical mapping has proven to be a fundamental experimental tool in the study of spatially extended electrophysiological heterogeneity of the cardiac substrate, without which appreciation of the complexity of this model wouldn't have been possible.

\subsubsection{APD Prolongation and Repolarization Reserves}

In their review, Tomaselli and Zipes approach APD prolongation as "the" cause of SCD in HF289. The underlying molecular basis of APD prolongation is expected to be multifactorial, however alterations in repolarization reserves ${ }^{x v i i}$ or $\mathrm{Ca}^{2+}$ homeostasis are sufficient to cause APD prolongation and subject the patient to increased risk of SCD 32,289 . Prolongation of the APD is a hallmark of cells and tissues isolated from failing hearts (isolated myocytes ${ }^{290}$ or whole ventricular preparations ${ }^{291}$ ), independent of the underlying cause that originally lead to this clinical state ${ }^{292}$. More importantly, APD prolongation is heterogeneously distributed across the ventricles; therefore it is often associated with an exaggeration of physiological inhomogeneity in electrical properties forming a substrate for functional reentryxix and lethal ventricular arrhythmias ${ }^{291}$. The main repolarizing currents in a cardiac cells are the ones mediated by distinct $\mathrm{K}_{\mathrm{V}}$ channels ${ }^{\mathrm{xx}}$. The relative density of these currents and their response to stress varies considerably in different regions of the heart. Hence prolongation of APD, when associated with down-regulation of repolarizing and upregulation of depolarizing currents lead to spatially and temporally labile repolarization that may predispose to afterdepolarization-mediated triggered activity and functional reentryxxi.

\subsubsection{Triggered Activity and Ectopic Conduction the Heart Muscle}

There is a wide agreement among investigators that abnormal impulse generation in susceptible cells could initiate arrhythmia. Triggered activity is one example, where membrane voltage oscillations are generated as a consequence of a prior normal $\mathrm{AP}^{293}$. Triggered activity travels more slowly across the myocardium than a normal impulse, secondary to the infringement of the electrical excitation on repolarization ${ }^{294}$. These afterdepolarizations were generally described by Cranefield almost forty years ago and classified into two categories: early and delayed, relatively to the phase of the AP where the intrusion occurs ${ }^{295}$. The strong association between triggered activity

xvii Refer to section 1.2.1 for a detailed description of this gap junctional protein.

xviii Loss of a $\mathrm{K}^{+}$current can impair repolarization, but there is a redundancy of $\mathrm{K}^{+}$currents in a normal cardiac cell so that when one $\mathrm{K}^{+}$current is dysfunctional, other $\mathrm{K}^{+}$currents increase to compensate, a phenomenon called repolarization reserve (Reference: Circ. Res. (1995) 77:140-152).

xix Refer to section 1.3.7 for a description of the difference between the main types of reentry: anatomical and functional.

xx Refer to section 1.1.4 for a detailed description of repolarizing currents in a cardiac myocyte.

xxi See section 1.3.5 below for a detailed description of EAD and DAD, and their role in triggered activity. 
and arrhythmogenic substrates leaves little uncertainty that such events, when occurring in the susceptible cell, could lead to complex activation patterns on the myocardial tissue level296 and to arrhythmias at the organ level297, 298. Nevertheless, despite this temptation the translation of abnormal cellular activity to the 3D multicellular heart isn't that straightforward. When an activity propagates the excitation wave front is a source of depolarizing current for the quiescent tissue to reach threshold and depolarize ensuring wave propagation, otherwise conduction block occurs due to large source-sink mismatch ${ }^{299}$. Hence the imperative aim prevails to understand the underlying ionic subcellular mechanisms, the role of cellular coupling in the amplification of single cellular events and the effect of functional heterogeneity in facilitating overt ventricular arrhythmia in the heart.

Different ionic mechanisms have been attributed to describe the proclivity of a cell to develop an early (EAD) or delayed afterdepolarization (DAD), which may lead to cardiac arrhythmias in a variety of clinical settings ${ }^{300}$. EADs are secondary depolarizations that occur during the repolarizing phase of the cardiac AP (end of phase 2, early phase 3), particularly prominent with APD prolongation, slow heart rate and QT-interval prolongation on the ECG ${ }^{301}$. EADs have been induced experimentally in isolated cardiac tissues under a variety of conditions that either enhance inward currents or reduce repolarizing currents (including bradycardia ${ }^{297}$, hypokalemia ${ }^{302}$, 303, reduced (acidic) $\mathrm{pH}^{304}$, hypocalcemia ${ }^{305}$, hypoxia ${ }^{306}$ and the use of some AADS, such as procainamide ${ }^{307}$ ), which could intuitively explain repolarization delay but does not necessarily explain the timedependent voltage oscillations that are characteristic of EADs. In generic terms, it's currently believed that any mechanism with the capacity to regeneratively increase net inward currents and by doing so compromises the myocyte's ability to repolarize could trigger EADs ${ }^{308}$. In the voltage range of EADs, two currents could potentially fulfill these properties: $I_{C a, L}$ and $I_{N C X}$. Although at the beginning of repolarization $\mathrm{Ca}^{2+}$ is still driven inward electrochemically, $\mathrm{I}_{\mathrm{Ca}, \mathrm{L}}$ eventually inactivates through a $\mathrm{Ca}^{2+-} \mathrm{CaM}$ dependent mechanism ${ }^{x \times i i}$, once $\left[\mathrm{Ca}^{2+}\right]_{\mathrm{i}}$ has significantly declined. Normally, $\mathrm{I}_{\mathrm{Ca}, \mathrm{L}}$ inactivation is slow $(\sim 100 \mathrm{~ms} \text { at } 0 \mathrm{mV})^{77}$, but it's accelerated at hyperpolarized potentials. Under circumstances where repolarization reserves are compromised and the plateau phase has lingered long enough at voltage ranges that facilitate recovery from inactivation ${ }^{308}$, $\mathrm{I}_{\mathrm{Ca}, \mathrm{L}}$ will regeneratively increase to reverse repolarization and trigger the EAD upstroke ${ }^{309}$. $\mathrm{I}_{\mathrm{NCX}}$ could play either a primary or complementary role in triggering EADs ${ }^{255}$. During phase 2 and 3, $\mathrm{I}_{\mathrm{NCX}}$ performs in the direct mode with a net inward current opposing repolarization, however not sufficient enough to reverse it unless $\left[\mathrm{Ca}^{2+}\right]_{\mathrm{i}}$ increases. Whenever the initial $\left[\mathrm{Ca}^{2+}\right]_{\mathrm{i}}$ increase is mainly due to reactivation of the $\mathrm{L}-$ type $\mathrm{Ca}^{2+}$ channels (as explained above), the role of NCX is considered complementary in facilitating EAD-triggered activity. Under other pathological conditions, $\left[\mathrm{Ca}^{2+}\right]_{\mathrm{i}}$ could remain sufficiently high (due to SR leakage for instance), promoting primarily NCX activity in the direct mode, hence prolonging the plateau long enough to allow L-type $\mathrm{Ca}^{2+}$ channels to recover from inactivation and trigger an EAD upstroke. The synergistic action of both currents is of clinical importance, since targeting either current alone may be sufficient to suppress EAD triggered oscillations ${ }^{308}$.

DADs are initiated at $V_{\text {rmp }}$ after the preceding AP has fully repolarized, and are widely accepted to be caused by $\mathrm{Ca}^{2+}$ overload, such as during spontaneous diastolic SR $\mathrm{Ca}^{2+}$ release events ${ }^{162}$. Unlike EADs, DADs are enhanced by tachycardia and slow heart rates suppress DAD formation 310 , where experiments using catecholamines 298 , high digitalis concentrations 298,311 and hypercalcemia 312 showed an increased frequency of DAD-triggered activity. These voltage oscillations may be in the subthreshold range ${ }^{313}$, but with sufficient increase $\left[\mathrm{Ca}^{2+}\right]_{i}$ could largely amplify $\mathrm{Ca}^{2+}$-dependent inward currents, mainly $\mathrm{I}_{\mathrm{NCx}}$, and $\mathrm{V}_{\mathrm{mbr}}$ can depolarize to threshold values leading to DAD-triggered APs. In this regard, EAD induction with $\mathrm{I}_{\mathrm{NCX}}$ being primarily involved appears to be mechanistically

xxii Refer to section 1.1.5. 
similar to DAD initiation ${ }^{314}$. Because DAD amplitude increases as the preceding cycle length decreases, if one afterdepolarization reaches threshold and causes premature firing, it is highly probable that the next depolarization reaches threshold and initiates a response, reinforcing a selfsustaining rhythm ${ }^{300}$. As described earlierxxiii, a substantial amount of independent couplons must be activated simultaneously in order for contraction to occur, since $\mathrm{Ca}^{2+}$ doesn't diffuse from one cleft to another triggering $\mathrm{CICR}^{163}$. Hence, the spontaneous $\mathrm{SR} \mathrm{Ca}^{2+}$ release probably needs to propagate in the cell as a $\mathrm{Ca}^{2+}$ wave for an after-contraction to be generated.

\subsubsection{Excitability: A Main Determinant of Conduction in the Heart}

A major factor affecting the propagation of the cardiac impulse is the magnitude of the rapid inward Na-current $\left(\mathrm{I}_{\mathrm{Na}, \mathrm{f}}\right)^{315}$. A decrease in this current is expected to decrease both the maximum rise of the AP upstroke $(\mathrm{dV} / \mathrm{dt})_{\max }$ and $\mathrm{CV}^{315}$. For a single cardiac cell under space-clamp conditionsxiv, a proportionality relation between $(\mathrm{dV} / \mathrm{dt})_{\max }$ and $\mathrm{I}_{\mathrm{Na} \text {, }}$ exists: all $\mathrm{Na}^{+}$ions entering the cell upon depolarization are used to discharge the local capacitance ${ }^{254}$. This proportionality is expected to be lost in the propagating AP in a multicellular network, because the depolarizing charges are divided between discharging the local capacitance and depolarizing the membrane of downstream cells ${ }^{254}$. One factor that directly governs $\mathrm{CV}$ in the cardiac tissue is the rate and amplitude of depolarization. Arsndorf defines cardiac excitability as an intuitive term that describes the ease with which cardiac cells undergo individual and sequential depolarization and repolarization repetitively, communicate with each other and propagate the electrical signal ${ }^{316}$. These events can be summarized in a matrix of electrophysiological properties, which includes the major determinants of normal cardiac excitability such as $V_{\text {rmp }}$, active and passive cellular properties ${ }^{316}$.

Ventricular cardiac cells lose excitability with more positive $V_{\text {rmp }}$ and generate depressed fast responses (i.e. an AP with reduced amplitude and maximum upstroke velocity $(\mathrm{dV} / \mathrm{dt})_{\max }$ ), a direct consequence of decreased $\mathrm{Na}_{\mathrm{v}} 1.5$ availability. A less negative $\mathrm{V}_{\mathrm{rmp}}$ delays $\mathrm{Na}_{\mathrm{v}} 1.5$ recovery from inactivation causing refractoriness to lag far behind the end of repolarization, in contrast to the normal case where recovery of excitability should be completed by the end of repolarization ${ }^{317}$. Thus cells with decreased excitability may not respond as fast to the next stimulus after the end of repolarization, facilitating conduction block as the need for a shorter time to complete repolarization increases, such as with increased heart rate ${ }^{318}$.

Classically, rhythm disturbances have been classified into one of the primary abnormalities in electrical activity: Either an abnormality of impulse generation, impulse propagation or both ${ }^{313}$. It's now widely acceptable to assume that under appropriate conditions, most if not all cells in the heart can become foci of ectopic beats and entrain the heart away from the normal sinus rhythm ${ }^{293}$. Unidirectional block and reentry have been described as the underlying mechanisms behind abnormalities in impulse propagation. Since the refractory period of a cardiac AP is relatively long $(\sim 200 \mathrm{~ms})$ and CV in cardiac muscle reasonably fast $\left(\sim 0.5 \mathrm{~m} . \mathrm{s}^{-1}\right)$, it was assumed that the loop of tissue around which reentry occurs needs to be quite long ${ }^{293}$. Thereafter, studies on depressed responses in Purkinje fibers revealed that under specific conditions cardiac impulses might become sufficiently slow to support reentry ${ }^{318}$. It was generally concluded that all that is essential for the occurrence of reentry was unidirectional block, i.e. some asymmetry in the rate at which membrane properties change as a function of distance, and conduction slowing 318 . With the emergence of the

xxiii Refer to section 1.1.5.

xxiv The equation relates the transmembrane potential $V_{m b r}$ to the total transmembrane ionic current $\left(I_{N a, f}\right)$ at the upstroke and states that the changes in $V_{\text {mbr }}$ occur due to displacement of charges on the membrane capacitance by the movement of Na-ions across the cell membrane, where $\left(d V_{m b r} / d t\right)_{\max }=-1 / C_{m b r}$. $I_{N a}\left(C_{m b r}=\right.$ membrane capacitance) (Reference: Kleber and Rudy. Physiological Reviews (2004) 84:431-488). 
work by Spach and colleagues in 1980xxv, addressing the discrepancies found between the continuous propagation theory and experimental data on conduction in the cardiac tissue, coupling excitability, the rate of rise of the upstroke and CV has become more complicated than expected ${ }^{319}$.

\subsubsection{The Concept of Reentry}

Under normal physiological conditions, the conducting impulse dies out after sequential activation of the atria and ventricles because depolarization is followed by a period of refraction, where the tissue enters temporarily inexcitability awaiting the subsequent activation arising from the SA node ${ }^{21}$. Reentry in simple terms means that "the propagating impulse doesn't die out after complete activation of the myocardium, but persists to reexcite the cardiac tissue once it recovers from the effective RP", entraining the myocardium far from the control of the SA node ${ }^{320}$. The atrial effective $\mathrm{RP}$ is approximated around $150 \mathrm{~ms}$ in human hearts, whereas the ventricular one is much prolonged reaching $500 \mathrm{~ms}^{321}$. An impulse destined to cause reentry must therefore survive the effective RP by resuming conductance in an isolated pathway, which could provide a return route to the regions that have been previously excited and must be sufficiently long to permit propagation of the impulse throughout the RP28. Such pathway with a normally propagating impulse would not likely to exist in the heart. However reentry would still be likely to occur, if such an impulse is sufficiently slowed down, that the wavelength would be significantly shorter 28,322 . Such a pathway could physically exist as an anatomical substrate for reentry, in conditions where fibrosis could potentially isolate a part of the cardiac tissue from the rest of the heart 254 . However, structurally normal heart could conceivably harbor functional substrates for reentry, where the CV of the traveling impulse and the RP of the designated cells characterize the wavelength of the circuit, rather than a well-defined anatomical barrier ${ }^{254}$.

\subsection{The Cardiac Sodium Channel Nav1.5}

The central selective pressure for voltage-gated sodium channels ( $\mathrm{Nav}$ ) has consistently been preserved throughout evolution, an element particularly preferred by nature when high-speed coordination and communication within higher organisms is of essence ${ }^{78}$, hence their expression in excitable tissues (brain, heart, skeletal muscle, neuroendocrine cells... etc.). A canine Purkinje myocyte expresses more than 100,000 $\mathrm{Na}^{323}$, but only 20,000 L-type $\mathrm{Ca}^{2+}$ channels ${ }^{324}$ and relatively fewer relevant replicates of each $\mathrm{K}_{\mathrm{V}}$ family ${ }^{78}$. $\mathrm{Na}_{\mathrm{V}}$ also have a shy presence in non-excitable cells, however their physiological role remains unclear 325 . They are the founding members of the $S 4$ superfamily of voltage-gated channels 326,327 , in terms of their discovery as a protein and characterization of their genetic and amino acids sequences. The standardized nomenclature for the different $\mathrm{Na}_{\mathrm{V}}$ was developed by Goldin et al.328, based on a numerical system that defines subfamilies and subtypes relying on similarities between the amino acid sequences of the channels. For instance, the name of an individual channel consists of the chemical symbol of the principal permeating ion $(\mathrm{Na})$. The subscript in $\mathrm{Na}_{\mathrm{v}}$ refers to the main physiological sensor or regulator (in this case: voltage). The number following the subscript indicates the gene subfamily (currently only $\mathrm{Na}_{v} 1$ for all $\mathrm{Na}_{\mathrm{v}}$ isoforms), and the number following the full point identifies the specific channel isoform (e.g., Nav1.1). This last number has been assigned according to the approximate order in which each gene was identified. Nine mammalian $\mathrm{Na}_{\mathrm{v}}$ have been identified, and their amino acid sequences aligned for a direct comparison, to possibly gain insights on their evolutionary origins. $\mathrm{Na}_{v} 1.1, \mathrm{Na}_{\mathrm{v}} 1.2, \mathrm{Na} \mathrm{a}_{1.3}$, and Nav1.7 are highly tetrodotoxinxxvi (or TTX)-sensitive and are broadly

xxv Discontinuous propagation in the cardiac tissue was addressed in detail in section 1.3.1.

xxvi Tetrodotoxin (TTX) is a potent neurotoxin, named after the order of fish from which it is most commonly associated, the Tetraodontiformes (tetras-four and odontos-tooth), or the tetraodon pufferfish. TTX inhibits Nav channels in a highly potent and selective manner. It blocks $\mathrm{Na}_{\mathrm{v}}$ only from outside of the nerve membrane, and is due to binding to the 
expressed in neurons. Their genes are all located on human chromosome $2 \mathrm{q} 23-24$, consistent with a common evolutionary origin. On the other hand, $\mathrm{Na}_{v} 1.5, \mathrm{Na}_{v} 1.8$, and $\mathrm{Na}_{v} 1.9$ are also closely related and their amino acid sequences are greater than $64 \%$ identical to those of the four sodium channels encoded on chromosome $2^{325}$. Their genes are located on human chromosome 3p21-24, consistent with a common evolutionary origin ${ }^{326}$. These channels unlike the ones on chromosome 2 are TTXresistant to varying degree. The reason behind Nav1.5 insensitivity is elaborated in section 1.4. The isoforms $\mathrm{Na}_{v} 1.4$ (chromosome 17q23-25), expressed primarily in skeletal muscle, and Nav1.6 (chromosome 12q13), in the central nervous system, are set apart from these other two closely related groups of sodium channel genes. They are considered to have a distant evolutionary relationship than the previous two groups, despite $84 \%$ similarity in genetic material when compared to the ones on chromosome $2^{329}$.

\subsubsection{Structure, Function and Conformational States}

The need to fully characterize the pathogenesis of hereditary channelopathies with electrophysiological methods has further strengthened the need to understand the structurefunction relationship of ionic channels. Almost all voltage-gated ionic channels are highly selective: i.e. their conducting pore is mostly permeable to a single ionic species (for instance, we describe Nav1.5 permeability to cations as such: $\left.\mathrm{Na}^{+}>\mathrm{K}^{+}>\mathrm{Ca}^{2+}\right)^{325}$. It's not yet clear how particular residues could confer a selectivity of more than 100:1 in favor of $\mathrm{Na}^{+}$over other cations, a remarkable feature considering an ionic transport rate that exceeds $10^{7}$ ions per second 78 . Among cation selective voltage-gated channels, the structure of the pore, the selectivity filter, the activation and inactivation gates are highly conserved, that structure-function can almost be deduced from one channel to the next ${ }^{330}$. In simple terms the process that accounts for the opening and closing of ionic channels is called gating ${ }^{73}$. In general, voltage-dependent channels can assume two conductance states: open or closed. During repolarization, the opening probability of $\mathrm{Na}_{v} 1.5$ is normally extremely low, i.e. only a few channels open randomly. Depolarization enhances activation by increasing the opening probability of the channel, when the channel is initially in the resting state and fully deactivated ${ }^{91}$. Upon depolarization, several voltage-dependent sequential steps, involving the movement of gating charges through the membrane, are needed to open the activation gate and move the channel from its resting state to the conducting one ${ }^{331}$. The channel's intrinsic inactivation occurs within a few $\mathrm{ms}$, and under unclamped conditions, it basically leads to membrane repolarization even in the absence of any $\mathrm{K}_{\mathrm{V}}^{330}$. Unlike activation, which requires several voltagedependant steps preceding the channel conducting state, the inactivation gate closes relatively slowly after depolarization in a single step ${ }^{98}$. For some ionic channels, conductance is sustained as long as membrane depolarization continues. For the Nav1.5 channel, maintenance of depolarization beyond the activation time of the channel reduces open probability by channel inactivation that is no longer voltage-dependent but time-dependent, leading to a closed state from which the channel cannot be activated ${ }^{332}$.

selectivity filter resulting in prevention of sodium ion flow. It does not impair the channel gating mechanism. More recently, TTX-resistant $\mathrm{Nav}$ have been discovered in the nervous system and received much attention because of their role in pain sensation (Reference: Proc Jpn Acad Ser B Phys Biol Sci. 2008;84(5):147-154). 
a

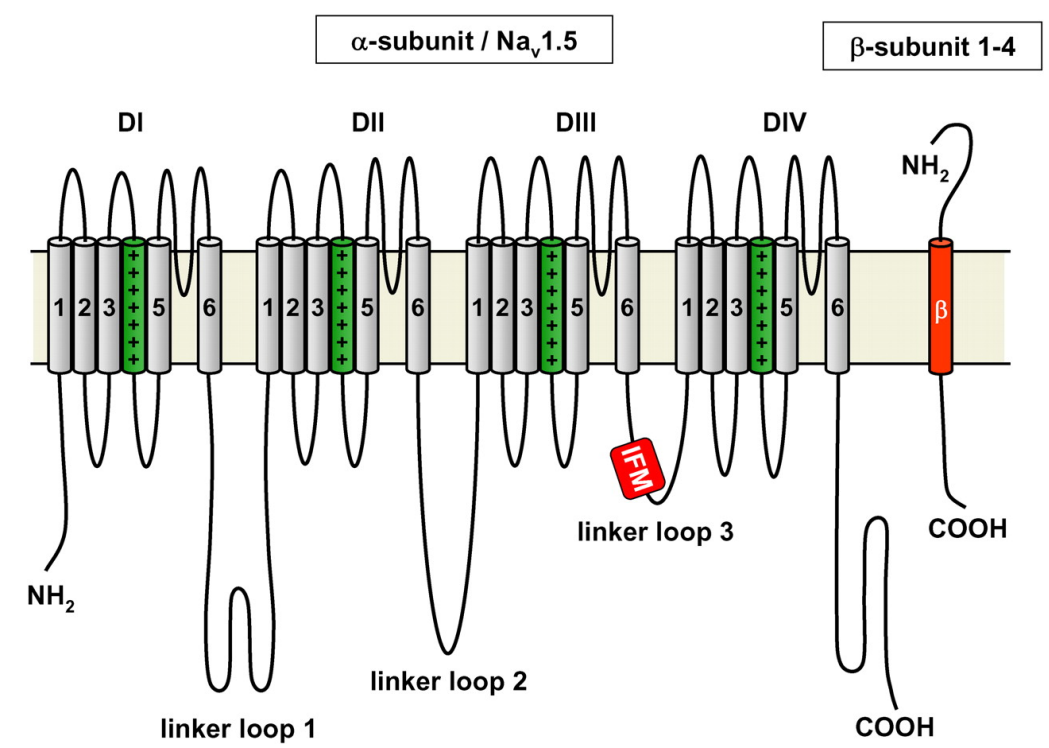

b

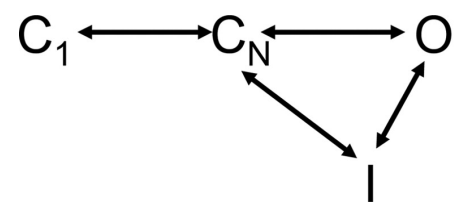

$\mathrm{C}_{1} \equiv$ Initial closed state

$\mathrm{C}_{\mathrm{N}} \equiv$ Closed state before the O state

$\mathrm{O} \equiv$ Open state

I $\equiv$ Inactivated state

Figure 4. $\mathrm{Na}_{\mathrm{v}} 1.5$ sodium channel and different transient states. a. A schematic topology of the $\mathrm{Na}_{\mathrm{v}} 1.5$, the pore forming alpha-subunit and its associated beta-subunits ${ }^{3}$. $\mathrm{Na}_{\mathrm{v}} 1.5$ consists of 4 domains (DI-DIV), each containing 6 transmembrane segments (S1-S6). All S4 segments are positively charged and act as voltage sensors, the concerted movement of which represents "voltage dependant gating" that precedes activation. The 4 domains fold around an ionconducting pore (not shown), which is lined by the loops of the S5 and S6 segments. The beta subunit is composed of one transmembrane segment. The DIII-DIV interlinker contains the IFM motif that plays a major role in fast inactivation. b. The state diagram as proposed by Leblanc et al. for the sodium channel ${ }^{7}$. The initial closed state the resting state, while $C_{N}$ is the state that precedes the complete conductance activated state 0 . Channel enters the inactive state after depolarization and doesn't open before recovery from repolarization occurred. The C-I transition may occur from multiple closed states, however resolving the kinetics between them is difficult to do with certainty, because they're non-conducting7. This transition is bidirectional and leads to cyclic connections of states, by the restraint of microscopic reversibility ${ }^{11}$. If $\mathrm{V}_{\mathrm{mbr}}$ is abruptly returned to its hyperpolarized resting value while the channel is open, it closes by deactivation, a reversal of the activation process. Pictures taken from references as noted.

The apparent voltage-sensitivity of inactivation comes from the fact that the receptor of the inactivation particle becomes available only when the activation gate is fully or partially activated, rendering inactivation directly coupled to activation ${ }^{333}$. This inactive state is a non-conducting state, different from the one taking place at the resting membrane potential, since inactivated channels don't respond to membrane depolarization. Among the Nav1.5 channel population, some channels move from the resting closed state to the inactive state without ever opening, a transition termed closed-state inactivation ${ }^{332}$, a fundamental gating process that critically distinguishes $\mathrm{Na}_{v} 1.5$ from other $\mathrm{Na}_{v}$ isoforms. Both neuronal and skeletal $\mathrm{Na}_{v}$ exhibit far less closed-state inactivation, hence remain excitable at more depolarized resting $V_{m b r}$ in their respective tissues ${ }^{100}$. The rate of channel opening may be maximal at the onset of $V_{\text {mbr }}$ change or delayed, with direct consequences on the shape of the mediated current. The number of closed states that precede channel opening determine the activation kinetics, i.e. the occurrence of multiple closed states leads to delays in activation ${ }^{77}$. To reverse inactivation, the membrane potential must repolarize, for the $\mathrm{Na}_{v} 1.5$ to recover from inactivation, a highly voltage-dependent mechanism accelerated by hyperpolarization. This is 
functionally crucial for the stability of repolarization; otherwise any influx of $\mathrm{Na}^{+}$through a conducting channel would be capable of inducing depolarization, since the recovery process takes place at negative voltages ${ }^{333}$. The steepness of voltage dependence of opening varies among voltagegated channels: the sodium channel increases its activation kinetics by e-fold (2.73) for $4 \mathrm{mV}$ change in $V_{m b r}$ depolarization, whereas the potassium channel increases by an e-fold for $5 \mathrm{mV}$ depolarization ${ }^{146}$.

Some 40 years ago, Hodgkin and Huxley $(\mathrm{HH})$ designed a mathematical model that accounted for the different transients of the $\mathrm{Na}^{+}$channel ${ }^{86}$. More complicated models have surged in parallel with more extensive and complex electrophysiological techniques ${ }^{45,334}$. Despite these advances, the core of HH-model is still considered valid 335 . The described sodium conductance being proportional to $m^{3} . h$, where $m$ (activation) and $h$ (inactivation) are voltage- and time- dependent variables, with values ranging between 0 and 1 ; as $m$ increases, $h$ decreases otherwise no current flows. When $h \sim 0$, inactivation is complete ${ }^{11}$. At resting state, the $m$ is small, $h$ is relatively large, such that the product $m^{3} . h$ is small and counteracts the fourth power of the activation variable $n$ of the $\mathrm{K}_{\mathrm{v}}$ channel. Resting channels have the $m$-gate in the closed position, whereas the $h$-gate in the open position. With both gates in the open position, $\mathrm{Na}^{+}$moves into the cell. Even with maintained depolarization, the $h$-gate moves into the closed position, terminating the $\mathrm{I}_{\mathrm{Na}, \mathrm{i}}$. With repolarization, the $m$-gate assumes the closed position (a process of only a few ms). Relatively, a substantial amount of time is needed before the $h$-gate can assume the open position (a process of $\sim 100 \mathrm{~ms}$ ) 335 .

Over the last 20 years, the molecular analysis of the sodium channel has made enormous progress, that was most recently described by Caterall 326 . It might suffice here to summarize the basic findings. The human cardiac $\mathrm{Na}_{\mathrm{V}}(\mathrm{hH} 1)$ is composed of an $\alpha$-pore forming subunit (encoded by the gene $S C N 5 A$ ) and associated $\beta$-subunits (only the heart and brain has 4 subunits bound to the $\alpha$ subunit by disulfide bonds ${ }^{336,337}$ ). The pore-forming $\alpha$-subunit is sufficient for functional expression, but the kinetics and voltage dependence of channel gating are modified by the subunits, which also play a role in channel localization and interaction with cell adhesion molecules, ECM, and intracellular cytoskeleton. Although $\mathrm{Na}_{v} 1.5$ is the predominant sodium channel in the mammalian myocardium, studies revealed that notable mRNA encoding Nav1.1, Nav1.3 (both predominantly expressed in the brain) ${ }^{338,339}$ and $\mathrm{Na}_{1} 1.4$ (skeletal muscle) ${ }^{340}$ are also expressed in the heart. Their physiological effect on the myocardium is still elusive, except for one report where TTX application leads to APD shortening in canine Purkinje fibers, which could indicate a possible role of these isoforms in the generation of $\mathrm{I}_{\mathrm{Na}, \mathrm{p}}$, the persistent component of the sodium current ${ }^{341}$.

Structure and function of the $\boldsymbol{\alpha}$-subunit. This pore forming subunit is a tetramer (domains I to IV, $\sim 260 \mathrm{kDa}$ ), where each domain is composed of 6 transmembrane $\alpha$-helices (S1-S6) connected by intra- and extra- cellular loops, the interlinkers ${ }^{342,343}$ (Figure 4a). The $\alpha$-subunit determines the main characteristics of ion selectivity, ion-conducting pore, the voltage sensors, gates and binding sites of endogenous and exogenous substances ${ }^{330}$. All $\mathrm{Na}_{\mathrm{v}}$ channels $\alpha$-subunits are subjected to posttranslational modifications such as glycosylation, an essential step for the maintenance of cellsurface expression of $\mathrm{Na}_{v}$ channels in excitable cells ${ }^{344}$. Unlike other $\mathrm{Na}_{v}$ channels, which are heavily glycosylated with more than $40 \%$ of their weight attributed to carbohydrates 345 , only $5 \%$ of the $\mathrm{Na}_{v} 1.5 \alpha$-subunit's weight is sugar 346 . The reason behind such a discrepancy is still unknown. The linkers between segments S5 and S6 in each domain (P-loops), denoted as SS1-SS2 (the N-terminal part and C-terminal part respectively), form the pore of the channel. A combination of site directed mutagenesis and toxin binding essays showed that neutralization of the negative residues found in the P-region abolished the ability of positively charged toxins to block the channel, which suggests that the P-region contributes to the lining of the pore ${ }^{347}$. Initially, the activation gate was hypothesized to physically exist on the internal side of the permeation pathway, because pore lining 
residues remain accessible to externally applied reagents regardless of the conformational state adopted by the channel ${ }^{348}$. Targeted mutations of the S6-segments altered $\mathrm{Na}_{\mathrm{V}}$ gating and responses to different drugs ${ }^{349}$. In later studies done on the $\mathrm{K}_{V}$ channel, the $\mathrm{S} 6$ segments were found to physically occlude the inner channel mouth upon changes in voltage ${ }^{350}$; and when associated with the previous results, this finding suggests the highly probable role of S6 segments acting as the physical activation gate ${ }^{78}$. Selectivity filters are common structures in voltage-gated channels. A few well-targeted alterations in the P-region could shift the channel's selectivity 351 : substitution of lysine and alanine residues with glutamate made the Nav1.5 channel selective for calcium ${ }^{352}$.

The S4 $\alpha$-helices in all four domains have a remarkable repetitive structure and carry arginine or lysine at every third position, the intervening residues being hydrophobic ${ }^{343}$. Nevertheless, unlike the $\mathrm{K}_{V}$ channels, the number of positive charges carried by each segment isn't similar and the contribution of each of the four S4 segments to activation is markedly asymmetrical 353,354 . When the channel is assuming the resting state, all the S4 segments are pushed inward by the negative voltage, which physically prevents the opening of the activation gate 355 . The S4 segments being the voltage sensors of the $\mathrm{Na}_{\mathrm{v}} 1.5$, a conserved structure among most voltage-gated channels ${ }^{356}$, move in a concerted stepwise outward manner during activation; therefore induce pore opening 353,355 . Even when the channel is blocked pharmacologically, the movement of these charged segments in the electrical membrane is still measurable and is the basis of gating currents ${ }^{357}$. The asymmetrical dynamics of the S4 segments mediate the stepwise recovery from inactivation during repolarization as well, forcing closure of the activation gate and the dislocation of the inactivation particle from its receptor site and returning the channel into its resting deactivated state ${ }^{259}$.

Most of the $\alpha$-subunits expressed in the brain and the skeletal muscles are sensitive to tetrodotoxin, which in $\mu \mathrm{M}$ concentrations blocks ion flux by binding to the aromatic residues of DI S5-S6 interlinker and occluding the external mouth of the pore. Nav1.5 is $\sim 200$ times less sensitive to this toxin, due to the presence of a cysteine residue at the equivalent position of the aromatic ones in the other $\mathrm{Na}_{v}$ isoforms ${ }^{358,359}$. Finally, local anesthetics (lidocaine), antiarrhythmic drugs (AADs) and anticonvulsants ${ }^{360}$ bind to the overlapping receptor sites located in the inner cavity of the pore. Amino acid residues in the S6 segments from at least three of the four domains contribute to this complex drug receptor site, with the DIV-S6 segment playing the dominant role ${ }^{326}$. The affinity of the receptor site to the blocking molecule has been the subject of a strenuous debate. Several assumptions regarding the mechanisms of drug-receptor interaction were made from early electrophysiological observations that anticipated state-dependency, from which two models were proposed 361,362 to explain the mechanism of action of AADxxvii. While these major electrophysiological hypotheses remain both elegant explanations for the data gathered from cellular studies, there has been renewed interest in the study of Class I AADs particularly after the discovery that Nav1.5 is by itself an elite target underlying many sudden cardiac death (SCD) syndromes.

Fast inactivation is defined by the rapid, almost simultaneous and complete decay of currents in response to the couple of ms depolarization. It derives most of its voltage dependence from coupling to activation ${ }^{11}$. During phase 2 of the AP in human ventricular myocytes, $\sim 99 \%$ of the $\mathrm{Na}_{V}$ population is in an inactivated, non-conducting state ${ }^{363}$. It's a distinctive form channel closure involving the outward motion of DIII-S4 and DIV-S4 in particular ${ }^{356}$. Intracellular introduction of pronase ${ }^{x x v i i i}$ abolished inactivation, which suggested the presence of a physical fast-inactivating gate

xxvii Refer to section 1.4.7, for further information regarding the two models: the modulated receptor hypothesis and the guarded receptor hypothesis that were proposed to explain AAD blocking mechanism.

xxviii Pronase: proteinsases isolated from the extracellular fluid. It's a protein-digesting enzyme. 
accessible from the cytoplasm ${ }^{364}$. Based on these results, Armstrong and Bezanilla (1973) proposed a hinged-lid model for the fast inactivation gate ${ }^{364}$. Mutagenesis experiments confirmed the Nachannel DIII-DIV interlinker to be the fast inactivation gate. In fact, substituting glutamine for glycine in that particular loop slows channel inactivation ${ }^{365}$, and leads to the life threatening potassium aggravated myotonia ${ }^{366}$. The inactivation particle itself consists of three consecutive amino acids, isoleucine, phenylalanine, and methionine forming the hydrophobic IFM motif or lid. Substituting IFM with a designed triplet of glutamine, abolished inactivation completely ${ }^{365}$. The hinges appear to be a pair of glycine and proline residues situated in the vicinity of phenylalanine.

Upon prolonged depolarization $\mathrm{Na}$ 1.5progressively enter the slow inactivation process or the coreinactivation (also called C-inactivation), a more stable non-conducting state that could range from hundreds of ms to many seconds 367,368 and is detectable even when fast inactivation has been abolished 11 . The molecular determinants of slow inactivation are not as well-understood as the ones of fast inactivation, however the S5-S6 loops (P-segments) seem to play a major role ${ }^{369}$. An intermediate state of slow inactivation $\left(\mathrm{I}_{\mathrm{M}}\right)$ develops in Nav1.5 with a time course relevant to the duration of depolarization 370 .

Structure and function of the $\boldsymbol{\beta}$-subunits. So far, $\mathrm{Na}_{v} 1.5$ (with other brain isoforms) has been identified to have 4 -associated $\beta$-subunits 337 (encoded by four genes $S C N 1 B$ to $S C N 4 B$ ) that modify channel function and mediate protein-protein interactions. All $\beta$-subunits contain a cleaved signal sequence, an extracellular immunoglobin-like fold, a transmembrane segment, and a short intracellular C-terminal tail. A notable difference is that $\beta_{1}$ and $\beta_{3}$ are noncovalently associated with the $\alpha$-subunit and have four cysteine residues in their immunoglobin-like fold ${ }^{371}$, whereas $\beta_{2}$ and $\beta_{4}$ have five extracellular cysteines, i.e. their fold contain an unpaired cysteine, which allows them to form disulfide bonds with the $\alpha$-subunit ${ }^{372}$. The $\beta$-subunits display several important functional properties. For instance, it was shown that $\mathrm{Na}_{v}$ loses its fast-gating kinetics, when the $\alpha$-subunit was expressed alone ${ }^{373}$ in Xenopus ${ }^{\text {xxix }}$ oocytes $^{374}$. Coexpression of the $\beta$-subunits (mainly $\beta_{1}$ and $\beta_{2}$ ) resulted in a substantial acceleration of the rate of inactivation ${ }^{372}$. Nevertheless, a $\beta_{4}$-subunit mutation was reported in a patient who presented with a LQTS3 electrocardiographic phenotype ${ }^{\mathrm{xxx}}$,

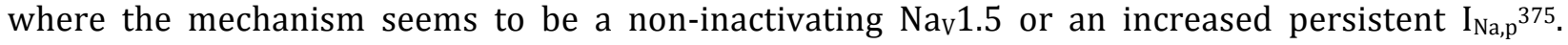
Three other mutations were identified in the $\beta_{1}$-subunit of Nav1.5 in a population of patients, who presented with Brugada syndrome (BrS) and idiopathic cardiac conduction disease (ICCD) phenotypes ${ }^{\mathrm{xxx}}$ without any mutation detected in the SCN5A gene ${ }^{376}$. $\beta$-subunit mutations of the brain channel isoform have been documented in human diseases such as generalized epilepsyxxxii with febrile seizures ${ }^{377,} 378$.

\subsubsection{Modulation of the Cardiac Na-Channel (Nav1.5)}

Meticulous opening and closing of cardiac ion channels result in generation of AP, propagation of the electrical excitation, then repolarizaton; an electrical activity is coupled to contraction and relaxation. Besides genetic mutations, developmental and acquired ones can ultimately affect the genetic expression of the tissue restricted Na-channels. For instance, abnormal electrical patterns or

xxix Expression cloning using Xenopus oocytes, since being pioneered in 1971, has been proven to be highly satisfactory tool for the structural and functional identification of proteins of all origins. The Xenopus oocyte is able to efficiently transcribe and translate injected genetic information, perform assembly of the foreign protein products, correctly process the resulting polypeptides, and target them to their proper subcellular compartments (Markovich, 2007).

xxx See section 1.4.3 for the description of long QT Syndrome, LQTS3.

xxxi See section 1.4.5 for the description of Brugada Syndrome (BrS) and idiopathic cardiac conduction disease (ICCD).

xxxii Generalized epilepsy with febrile seizures is a recently described epilepsy syndrome, comprising febrile seizures with childhood onset ( 1year), but unlike typical febrile convulsions, attacks with fever continue beyond 6 years of age and afebrile seizures start to cease by puberty. 
epilepsy can alter $\mathrm{Na}_{\mathrm{v}}$ gene expression in the brain 78,379 . Chronic exposure to Na-blocking AADs was found to increase steady state $\mathrm{Na}_{\mathrm{V}} 1.5 \mathrm{mRNA}$, which could tend to counteract the effects of channel blockade, a probable factor mediating resistance to treatment and facilitating proarrhythmic effects of AADs upon withdrawal ${ }^{380}$. Alterations in cell-cell coupling, heart disease congenital channel abnormalities, drug interventions or electrolyte imbalance could manifest themselves as an abnormal electrical activity. In channelopathies, mutations can affect the functional or accessory subunits or modulatory proteins ${ }^{381}$. In this thesis work, two models of Nav1.5 abnormalities are investigated: the first one is a model of Duchenne muscular dystrophy, where the Nav1.5 regulatory protein dystrophin is deficient (introduced in section 1.2.2); whereas the second is a model of longQT syndrome (LQTS), where the mutated protein is the sodium channel itself (introduced in section 1.4.3).

Electrocardiographic (or ECG) abnormalities. Morphological changes in cellular AP are translated into changes that appear on the body surface ECG, a signal that represents the vectorial projections of the overall cardiac spatial and temporal electrical gradients generated at different phases of the AP382. Atrial depolarization precedes ventricular depolarization and it's manifested as a low-frequency P-wave that precedes the peaked QRS signal, the latter is indicative of the high frequency components of the bulkier ventricular muscle depolarization ${ }^{1}$. The T-wave that follows the QRS complex represents ventricular repolarization. For instance, widening of the QRS complex beyond $120 \mathrm{~ms}$ (in adult humans) is indicative of conduction slowing across the ventricles. ST elevation correlates with increased transmural voltage gradients during phase 2 of the AP, a hallmark of myocardial injury, such as the one occuring during an acute episode of a heart attack (myocardial ischemia) ${ }^{383}$. APD prolongation results in a prolonged QT interval, which may or not be associated with $\mathrm{T}$ wave abnormalities, considerably increasing the risk of developing lethal arrhythmias, syncopexxxiii and SCD ${ }^{384}$.

\subsubsection{Genetic mutations of Nav1.5: The Long QT Syndrome}

LQTS is a collection of cardiac disorders characterized by a prolongation of the QT-interval on the ECG and can be divided clinically into two categories: primary and secondary 385 . Secondary LQTS generally results from either pharmacological interventions aiming at treating non-cardiac or cardiac diseases ${ }^{386}$, electrolytes disorders (hyperkalemia) ${ }^{387}$ or acquired cardiac diseases (cardiomyopathies, ischemia) ${ }^{385}$. Primary LQTS is predominantly due to inherited genetic mutations of the cardiac $\mathrm{K}^{+}$and $\mathrm{Na}^{+}$channels (and other regulating proteins), and so far more than ten genes have been implicated in the disease ${ }^{388}$. The variant 3 of LQTS (LQTS3) is caused by a mutation in the gene encoding for the $\alpha$-subunit of the cardiac sodium channel ${ }^{389}$. Eighty different SCN5A mutations have been identified in LQTS3 patients so far ${ }^{390}$, hence different mechanisms were described trying to explain QT interval prolongation.

The first genetic defect, positioned at the short arm of chromosome 3 (3p21-24) $)^{391}$, was detected in patients with an autosomal dominant form of LQTS3 ${ }^{392}$. This mutation is severe enough to cause a deletion of nine bases coding for lysine-1505, proline-1506 and glycine-1507 in the DIII-DIV intracellular linker ${ }^{392}$. The loss of those amino acids, termed as the $\triangle \mathrm{KPQ}$ mutation ${ }^{393}$, induces a disruption of the motif crucial for the fast inactivation of $\mathrm{Na}_{v} 1.5$, hence results in a persistent noninactivating current ${ }^{394}$, typically ranging between $0.5-2 \% \mathrm{I}_{\mathrm{Na}, \mathrm{i}} 395,396$. This gain of function or bursting mutation leading to altered gating highlights the critical role of fast inactivation in the cardiac Nachannel isoform in the preservation of the normal electrical function, when compared to its

xxxiii Cardiac syncope is defined as sudden loss of consciousness, with momentary premonitory symptoms or without warning, due to cerebral anemia caused by obstructions to cardiac output or arrhythmias such as ventricular asystole, extreme bradycardia (heart rate < 45-50), or ventricular fibrillation (Webster Medical Dictionary). 
muscular or neural counterparts that exhibit far less inactivated states ${ }^{73}$. First and foremost, activation of $\mathrm{Na}_{v} 1.5$ is sharply voltage-dependent. However, inactivation is also a voltage-dependent process and some of these channels normally undergo closed-state inactivation ${ }^{\text {xxxiv }}$, without ever opening upon membrane depolarization ${ }^{327}$. When membrane voltage lingers at depolarizing potentials such as the plateau phase, present estimates reveal that $99 \%$ of $\mathrm{Na}_{\mathrm{v}} 1.5$ are expected to be in the inactivated, non-conducting state 363,395 . The remaining $1 \%$ could be open during phase 2 of the AP and mediate the late inward Na-current $\left(\mathrm{I}_{\mathrm{Na}, \mathrm{late}}\right)^{\mathrm{xxxv}}$. The probability of $\mathrm{Na}_{\mathrm{v}} 1.5$ opening during this phase can be determined by the overlap of the steady states activation and inactivation Boltzman curves ${ }^{118}$. This voltage window has two important implications on the kinetics and molecular contingency of Nav1.5: First, there exists a finite possibility that inactivation is reversible at depolarizing voltages (i.e. a finite population of inactivated channels can reopen during the plateau). Second, an upregulation of the window current or $\mathrm{I}_{\mathrm{Na} \text {,late }}$ could inflict a calamitous prolongation of the $\mathrm{AP}^{106}$. This small amplitude current has been described in normal hearts, and appears to be amplified in disease models such as heart failure ${ }^{397}$. Eminently, the presence of an inwardly leaking $\mathrm{I}_{\mathrm{Na} \text {, late }}$ will counteract the repolarizing reserve, thereby either delaying

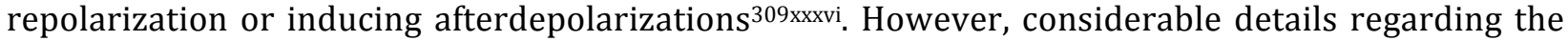
nature of $I_{N a, l a t e}$ remain unresolved. Does this current result from channels that failed to inactivate after the upstroke (i.e. this late current is a remnant or a sustained component of the fast inward $\mathrm{I}_{\mathrm{Na}, \mathrm{f}}$ ) or from channels that underwent reverse inactivation, or both?

Another example of altered gating was proposed when the behavior of channels harboring the E1295K mutationxxxvii didn't seem to match well with the mechanism induced by the $\triangle \mathrm{KPQ}$ mutation ${ }^{398}$. In this particular case of LQTS3, glutamic acid (E1295) was mutated into a positively charged lysine in the 4-amino acid extracellular loop connecting the S3-S4 segments of DIII. S4 is one of the voltage sensors for activation gating process of the channel ${ }^{356}$, and E1295 is the amino acid closest to DIII-S4326. Instead, the mutation induced a positive shift in the half maximal voltage of steady state inactivation and activation, a shift of the window current to voltages where the repolarizing currents are not freed from their rectification ${ }^{x x x v i i}$ constraints and hence unable to counteract the effects of Nav1.5 reopening398. A third mechanism implicated in LQTS3 was associated with the I1768V mutation, which also didn't point to a bursting effect of the Nav1.5 channel ${ }^{363}$, 399. This mutation, located within the DIV-S6 transmembrane segment, results in the substitution of valine instead of isoleucine ${ }^{363}$ and increases the availability of Nav1.5 during repolarization, by enhancing the capacity of the channel to recover from inactivation at a range of membrane voltages that actually favors the activation transition, i.e. at diastolic membrane potentials 400 .

\subsubsection{Spatial Dispersion of Repolarization: An Arrhythmogenic Mechanism}

Studies in the past two decades have shed the light on the natural heterogeneity that exists within the normal ventricular wall, which comprises of at least three electrophysiologically and functionally different cell types: epicardial, midmyocardial and endocardial ${ }^{401}$. The principal difference between these cells is believed to reside in the ionic machinery available for each type ${ }^{262}$, 402. When isolated, the resulting APDs appear to deviate significantly in magnitude ${ }^{268}$. However, this dispersion seems to smooth out in the intact myocardium, mainly due to electrotonic interactions

xxxiv Refer to section 1.4.1, for a description of the different conformational and functional states of Nav1.5.

xxxv Refer to section 1.1.4, for a description of the late Na-current and its infliction on AP prolongation.

xxxvi Refer to section 1.3.5, for a description of triggered activity and afterdepolarizations.

xxxvii The standard nomenclature to describe a point mutation is used such that $\mathrm{A}^{* * * *} \mathrm{~B}$ describes that an amino acid residue $\mathrm{A}$ is replaced by the residue $\mathrm{B}$ at position ${ }^{* * * *}(*$ is a number $)$.

xxxviii Ionic current rectification was introduced in section 1.1.4. 
between the different cells types, causing the gradual decrease in APD across the ventricular wall from endocardium to epicardium ${ }^{257}$. It's now widely accepted that the degree of electrical coupling, in addition to the intrinsic differences in APD determines the extent to which spatial dispersion of repolarization (SDR) is enunciated in the ventricular myocardium ${ }^{270}$. One of the arrhythmogenic mechanisms proposed for the LQTS substrate is amplification of the SDR, which when present in either early or late phases of the AP can lead to the development of a variety of arrhythmia 403 . Altered SDR has been associated with arrhythmogenicity in cases of electrolytes' imbalance (such as hypokalemia ${ }^{404}$ ) or in genetically manipulated hearts lacking repolarizing $\mathrm{K}^{+}$currents $^{405}$. Amplification of intrinsic heterogeneity together with premature impulses could principally underlie the mechanistic trigger for ventricular arrhythmias in cardiac pathologies such as heart failure 271 and "Torsades de pointes" in LQTS3 ${ }^{406 .}$

The concept of heterogeneous repolarization, as a facilitator of arrhythmogenic activity, has been recognized for more than four decades $407-409$. Even in the absence of any structural heterogeneity due to infarction for instance, intrinsic spatial variations in repolarization currents in the heart have been shown to produce spatial gradients in APD ${ }^{410}$. Experimental work, including mapping experiments using extracellular electrodes, have shown significant SDR in arrhythmogenic diseases, such as Long QT Syndrome (LQTS) and Brugada Syndrome261, 264. When transmembrane repolarization at some sites of the cardiac tissue outlasts repolarization at adjacent sites, local current will flow in proportion to the voltage gradient between the two sites ${ }^{411}$. If the current is of sufficient magnitude, it can reexcite the earlier repolarizing site by bringing the cells' potential to threshold. Hence, marked differences in APD have been suggested to induce a special type of reentry termed "focal reexcitation"30,407, 412 and "prolonged repolarization-dependent rexcitation" 413

\subsubsection{Other Mutations of Nav1.5 and Heterogeneous Phenotypes}

A distinct class, previously referred to as idiopathic ventricular fibrillation, has been attributed to $\mathrm{Na}_{\mathrm{v}} 1.5$ loss of function mutation ${ }^{414}$, and underlies the Brugada Syndrome (BrS) phenotype ${ }^{415}$. BrS has characteristic ECG manifestations with a right bundle branch block morphology ${ }^{\text {xxxix }}$, an STelevation in left precordial leads, associated with ventricular tachycardia and fibrillation resulting in SCD ${ }^{416}$. Unlike LQTS3, BrS characteristically causes a reduction in $\mathrm{I}_{\mathrm{Na}}$, which occurs through a variety of proposed mechanisms ${ }^{390}$ : reduced rates of recovery from inactivation (which decreases Nav1.5 availability for subsequent beats), enhanced inactivation subsequent to channel opening, and protein trafficking defects that alter $\mathrm{Na}_{v} 1.5$ localization in the sarcolemma ${ }^{417}$. Studies on the $\mathrm{BrS}$ $\mathrm{Na}_{\mathrm{V}} 1.5$ mutations revealed that a particular mutation (T1620M) increased the tendency of the channel to reside in the intermediate state of slow inactivation ${ }^{348}$, implying a loss of function attributed to the decreased availability of the channels at fast heart rates ${ }^{418}$.

Isolated cardiac conduction disease (ICCD) is a phenotype tracked down to a loss of function SCN5A mutation (G514C) in the DI-DII interlinker ${ }^{419}$. ICCD is characterized by a slowing of conduction throughout the myocardium, which manifests itself by a widening of the QRS complex, bradycardia and possibly syncope 419,420 . Heterologous expression ${ }^{x l}$ of the mutated channel uncovered a depolarizing shift in the voltage dependence of $\mathrm{Na}_{v} 1.5$ activation; hence stronger depolarization gradients are required to open the channel. This shift could likely result from an inherited insensitivity of $\mathrm{Na}_{\mathrm{v}} 1.5$ to $\mathrm{V}_{\mathrm{mbr}}$ changes delaying opening of the channel and pushing the peak of the $\mathrm{I}_{\mathrm{Na}, \mathrm{i}}$ to more depolarized membrane potentials ${ }^{419,420}$. Such a delay is expected to cause a ubiquitous

xxxix Right bundle branch block is attributed to a decrease in electrical conduction velocity in the Purkinje fibers forming the right bundle branch. It has a characteristic QRS morphology on the ECG.

${ }^{\mathrm{xl}}$ Heterologous expression is to express a gene or a part of a gene in a host organism, which under normal conditions doesn't express this particular gene or fragment. 
decrease in $(\mathrm{dV} / \mathrm{dt})_{\max }$, which most probably underlies the $15 \%$ depression in myocardial CV and contributes to the bradyarrhythmia phenotype ${ }^{419}$, currently treated with implantation of a pacemaker.

Further insights into the heterogeneity of pathological manifestations of diseases associated with $\mathrm{Na}_{v} 1.5$ abnormalities were gained from individuals who appeared to have heart-rate dependent LQTS and BrS features: the QT-interval is prolonged at slow hearts and a distinctive ST-elevation occurs during exercise or at faster heart-rates ${ }^{421}$. More interestingly, a single Nav1.5 mutation (179insD) with an aspartic acid insertion in the carboxylic terminal was shown to contribute to these opposite effects ${ }^{421}$, by affecting the two distinct kinetic components of Nav1.5 inactivation. The mutation disrupts the fast component of inactivation, generating a persistent $\mathrm{Na}^{+}$current $\mathrm{I}_{\mathrm{Na} \text {, }}$ $\left(\sim 1.4 \% \mathrm{I}_{\mathrm{Na}, \mathrm{i}}\right)^{370}$ throughout the plateau, hence prolonging the QT-interval at slow heart rates. In addition, it simultaneously enhances the intermediate kinetics ${ }^{422}$ of slow inactivationxli in depolarized $\mathrm{Na}_{v} 1.5$, during sustained depolarization, thereby reducing the availability of $\mathrm{Na}_{v} 1.5$ primarily at rapid heart rates, which could explain ST-elevation on ECG during exercise ${ }^{421,423 .}$

Substantial increase in the probability that the channel would slow-inactivate at depolarization pulses within the cardiac APD ( 100-200ms), implies that fewer channels would have recovered from inactivation at rapid heart rates, where hyperpolarized diastolic intervals are narrow 370 , a characteristic finding in loss of function mutations ${ }^{424}$. Notably, studies involving $\mathrm{Na}_{\mathrm{v}} 1.5$ gating in myocytes isolated from epicardial regions bordering a 5-day infarcted zone in the canine heart reveal a delay in recovery from inactivation, with enhancement $\mathrm{Na}_{v} 1.5$ loss of function is a proarrhythmic mechanism, which is not restricted to genetically transmitted electrical diseases of the heart, but could also be implicated in more common acquired forms of SCD, such as infarction ${ }^{100}$.

\subsubsection{Anchoring and Modulating Proteins}

Cardiac ionic channels trafficking, the mechanisms behind their regulation on different membrane domains and the diverse proteins modulating their function still pose largely unanswered questions ${ }^{14}$. Multidomain membrane proteins appear to affect $\mathrm{Na}_{\mathrm{v}} 1.5$, indulging in the possibility of several regulation processes that target the channel, depending on whether it's located in the ID or LM $^{425}$ (Figure 3c).

SAP97 (along with another similar protein SAP90) was isolated initially from synapses in the rat cerebellum ${ }^{426}$. In the heart, two isoforms of SAP97 are expressed, differing in the combination of spliced insertions that determine the subcellular localization of the protein ${ }^{427}$. It's present in both atrial and ventricular myocytes, however seems to be predominantly localized towards the longitudinal ends of the cardiac cells, near or at the ID 239 . Through cultured adult cardiac myocytes, where specialized cell-cell contacts can be followed in vitro, much evidence is gathered concerning the sequence of events that leads to SAP97 being targeted to particular cardiomyocytic domains ${ }^{206}$. Several cardiac ionic channels interact with SAP97239, 428, including $\mathrm{Na}_{\mathrm{v}} 1.5$, particularly via the PDZ domain ${ }^{429}$. PDZ is a protein-protein interaction domain ${ }^{430}$ and its name derives from the first letters of three proteins where this domain was initially identified 431 : PSD-95 (a 95kDa protein involved in synaptic signaling), Dlg (the Drosophilia melanogaster disk) and ZO-1 (zonula occludens 1 protein involved in the maintenance of epithelia cells polarity). The effect of SAP97 on the cardiac sodium

xli In the report by Veldkamp et al. (Circ. Res., 2000), the authors argue that $\mathrm{Na}_{\mathrm{v}} 1.5$ is likely to fill the intermediate state of slow inactivation $\left(\mathrm{I}_{\mathrm{M}}\right)$, which is induced by longer depolarization times $(\sim 100-200 \mathrm{~ms})$ than the ones used to detect the fast inactivation behavior $(\sim 10 \mathrm{~ms})$, but considerably shorter than the ones used to describe the classic slow inactivated $(\sim 60 \mathrm{~s})$. Hence their slow inactivation reporting falls within the intermediate kinetics of slow inactivation rather than the longer timescale slow inactivation. 
channel appears so far to be functional. Heterologous expression of SAP97 and cardiac ionic channels has been studied and their associated currents were measured using patch-clamping techniques. Enhancement of the corresponding current was observed with SAP97 expression ${ }^{238}$, however this effect couldn't be linked to changes in the channel's intrinsic kinetics, since SAP97 induced no change in the activation of the channel or in the open probability ${ }^{240}$. On the other hand, protein levels detected by Western blot assay were increased in cardiomyocytes co-expressing SAP97, indicating possibly an increase in channel density ${ }^{432}$. In studies where SAP97 expression was silenced, significant decrease in $\mathrm{I}_{\mathrm{Na}, \mathrm{i}}$ was recorded and decreased membrane excitability ensued ${ }^{238}$, 239. The mechanism by which SAP97 enhances the functional capacities of the Nav1.5 channel remains unknown ${ }^{14}$.

As described in section 1.2.2, dystrophin stabilizes the DSMC in the LM domain ${ }^{221}$. Dystrophin acquires multiple protein-protein interaction domains, but interacts with $\mathrm{Nav} 1.5$ via the syntrophin adaptor proteins ${ }^{433}$. The syntrophin protein interacts with a domain at the carboxylic end of dystrophin (its amino end interacts with the actin filament) and simultaneously, via its PDZ domain, interacts with the carboxylic tail of Nav1.5, at the channel's PDZ binding motif 237 . In the context of dystrophin loss, which mainly affects striated muscles, the regulatory role of dystrophin in electrophysiology was therefore hypothesized to be more substantial. Petitprez et al. suggested the possible existence of two distinct subpopulations of Nav1.5 channels with two different anchoring proteins: the channels located in the ID are most probably associated with SAP97, whereas the other subpopulation is predominantly anchored to the LM via the DSMC238.

In addition to SAP97 and the DSMC, studies have shown interactions between ankyrin and Nav1.5. It was previously thought that ankyrin's role is strictly structural, anchoring membrane ionic channels and other ATPases to the cytoskeleton ${ }^{434}$. But studies in the last ten years showed a more mediatory role of this protein and its possible involvement in ECC 435 . Both ankyrin $B$ and ankyrin $G$ are expressed in the heart ${ }^{434}$. They are differently localized and the mutations of which lead to completely different phenotypes. Ankyrin-B is found at the level of the M-line and the Z-disk ${ }^{436}$, while ankyrin-G was localized at the ID and t-tubules ${ }^{437}$. It was shown that many transmembrane ionic transporters, such as the Na-pump and NCX, were erroneously localized in cardiomyocytes of mice expressing only one allele of the ankyrin gene ${ }^{438}$. While ankyrin $\mathrm{B}$ has been linked to several pathologies such as LQTS4, atrial fibrillation and SCD, hence the name ankyrin-B cardiac syndrome ${ }^{439}$; decreased ankyrin-G expression was mainly associated with decreased Nav1.5 expression and consequently a decrease in its corresponding current. No such effects were reported for ankyrin-B ${ }^{14}$. Nevertheless, a missense mutation in the $\mathrm{Na}_{\mathrm{v}} 1.5$ channel leading to the Brugada syndrome phenotype was associated with a disruption of the interaction between the channel and ankyrin-G440.

Although the interaction site between caveolin and $\mathrm{Na}_{v} 1.5$ is still unknown ${ }^{14}$, immunostaining images show a colocalization of those two proteins ${ }^{198,441}$ and recent electrophysiological data have also emphasized the role of mutated caveolin in cardiac function. Caveolin is a transmembrane protein that recruits different signaling molecules and is an important constituent of sarcolemma invaginations, the caveolae. The cardiac isoform Caveolin-3 (CAV3) is recently described as a binding partner for the dystroglycan molecule, part of the DSMC ${ }^{442}$. Dystrophin was shown to be a component of caveolae, raising the possibility of an indirect interaction between CAV3 and Nav1.5 via the DSMC443. Mutations in the cardiac caveolin isoform (CAV3) were found in patients with fatal arrhythmogenic phenotypes such LQTS ${ }^{41}$, sudden infant death syndrome ${ }^{444}$, and familial hypertrophic cardiomyopathy ${ }^{445}$. When $\mathrm{Na}_{\mathrm{v}} 1.5$ was co-expressed with mutant CAV3, the persistent inward $\mathrm{I}_{\mathrm{Na}, \mathrm{p}}$ (see section 1.1.4) was shown to be increased, a consistent finding with LQTS arrhythmogenic mechanism ${ }^{441,444}$. 
In this section, only the major proteins that potentially affect $\mathrm{Na}_{v} 1.5$ regulation with the involvement of the DSMC were presented. For a complete overview of other interacting proteins that could possibly modulate $\mathrm{Nav} 1.5$, refer to the extensive review by H. Abriel ${ }^{446}$.

\subsubsection{Pharmacological Interventions: Antiarrhythmic Drugs}

In the past 40 years, considerable progress has been made in pharmacological therapy of arrhythmic disturbances of the heart ${ }^{7}$. The treatment of a large subset of arrhythmias is currently well managed and tolerated by patients ${ }^{133}$. However, sudden cardiac death (SCD) remains a major cause of mortality internationally, with more than 3 million people dying yearly ${ }^{447}$. Implantable cardioverter-defibrillators or ICDs have revolutionized the approach to the prevention of SCD by delivering anti-tachycardia pacing and high-energy shocks ${ }^{448}$. The availability of these modes of treatment has encouraged the shift to these devices, particularly in high-risk patients ${ }^{448}$. Although, they can be life saving, they can also deliver inappropriate shocks. Henceforth, pharmacological management remains the mainstay treatment in many cases of arrhythmia ${ }^{7}$, and as an adjuvant therapy in patients with ICDs ${ }^{449}$.

The targets of many antiarrhythmic drugs (AADs) are the ionic channels that mediate the corresponding currents to be modulated. Their side effects, mainly due to their limited selectivity, have forced a careful examination of the indications where these drugs can be used 450 . For instance, many $\mathrm{Na}_{v} 1.5$-blockers are not pure use-dependent $\mathrm{Na}_{v} 1.5$ antagonists, but also block $\mathrm{K}^{+}$and/or $\mathrm{Ca}^{2+}$ channels 451 . The recent cloning and sequencing of major cardiac ionic channels have emphasized their striking similarities. Hence, it's hardly surprising that these drugs can act on more than one channel at a time ${ }^{452}$. This poses a substantial challenge for an optimal treatment using pharmacological agents.

$\mathrm{Na}_{v} 1.5$-blockers primarily block $\mathrm{Na}^{+}$influx through the channel. This is usually measured by the reduction in the amplitude of the fast inward current ${ }^{x l i i}\left(\mathrm{I}_{\mathrm{Na}, \mathrm{f}}\right)$, the shallower upstroke velocity, and the slowing of conduction across the myocardium ${ }^{453}$. The strong use-dependence block ${ }^{\text {xliii }}$ (UDB) of some $\mathrm{Na}_{v} 1.5$ blocker, a property believed to be at the core of their proarrhythmic effects, has shifted the interest of investigators towards $\mathrm{K}^{+}$blockers ${ }^{451}$. The ideal $\mathrm{K}^{+}$blocker is to cause little APD prolongation and refractoriness at slow hearts, while producing progressively greater effects on those parameters at higher heart rates (use-dependence prolongation of APD and refractoriness) ${ }^{454}$. But in reality, most available $\mathrm{K}^{+}$blockers display the opposite behavior, limiting their efficacy, and predisposing patients to Torsades de Pointesxliv, due to excessive APD lengthening at slow heart rates ${ }^{131}$, 455 . To further illustrate the challenge in designing AADs, $\mathrm{K}^{+}$blockers, for example, act by altering the time course of repolarization and refractory period ${ }^{131}$. By doing so, they will facilitate reentry either way, by shortening or lengthening the phase 3 xlv of the AP455. On one hand, shortening phase 3 means reducing the time interval during which the impulse must linger to await recovery of excitability: acetylcholine-mediated tachyarrhythmia in the normal tissue is an example of such type of reentry 456,457 . On the other hand, prolonging phase 3 of the AP and the time needed for recovery,

\footnotetext{
xlii Refer to section 1.1.4 for a detailed description of the sodium current.

xliii Use dependence block is a term used to describe a property of Nav1.5 blockers being increasingly effective with frequent pulses until a new steady state is reached. This is a desirable effect, particularly in the presence of ventricular tachycardia. However, this same effect is also shown to be proarrhythmic, since unidirectional block can ensue favoring reentrant rhythms (Reference: Clin Chem (1989) 35(5): 748-754).

xliv Torsades de Pointes was first reported by Dessertenne in 1966, to describe a particular form of polymorphic ventricular tachycardia, with a characteristic of the QRS twisting around the isoelectric line (Reference: Am J Cardiol (1987) 59:10-18)

xlv Refer to section 1.1.4 for a description of the different phases of the AP.
} 
may lead to marked APD prolongation and slowing of conduction of premature impulses, which could also facilitate reentry $131,455$.

Use-dependence of Nav1.5 blockers has two major features: voltage-dependence and frequency dependence ${ }^{453}$. A drug is said to be voltage dependent, if its effect is significantly dependent on the resting membrane voltage $\left(\mathrm{V}_{\mathrm{rmp}}\right)^{453}$. For example, a drug might be less effective in blocking Nav1.5 channels in cases of depolarized $V_{\text {rmp }}(\sim-65 \mathrm{mV})$ compared to the normal case $(\sim-80 \mathrm{mV})^{\text {xlvi }}$. Conversely, frequency-dependence means that the drug is particularly more effective in blocking the channel during a rapid series of depolarizations, rather than one single long depolarization ${ }^{453}$. All Nav1.5 blockers are state-dependent ${ }^{73}$, i.e. they either block the channel in its open or inactivated statexlvii. Rested channels are assumed not to bind any drug. These apparent state-dependencies have prompted a series of investigation to model drug-channel interactions. Two major hypotheses attempted to describe this mechanism: the Modulated Receptor Hypothesis (developed by Hille ${ }^{361}$, Hondeghem and Katzung362, 458 in 1977) and the Guarded Receptor Hypothesis (developed by Starmer et al. in 1984459). The modulated receptor hypothesis proposes that drugs bind to a channel-associated receptor with state specific affinities, i.e. binding occurs with state-specific association and dissociation rate constants and drug-bound channels do not conduct a current even if activated ${ }^{453}$. According to this model, the ability of a drug to bind to a particular state of the channel depends on the time during which the channel occupies this state and state-specific rate constants 453 . While the guarded receptor hypothesis assumes that the drug binds to a constantaffinity receptor, whose access is regulated by the gating states leading to apparent variable affinities as the channel changes conformational states ${ }^{453}$. It shares several features with the modulated receptor hypothesis, however it explicitly states that the affinity of the receptor site for the drug molecule is constant (i.e. conformation independent), but channel gates restrict drug access to the receptor, thus controlling binding with the channel ${ }^{453}$. So far, both remain two attractive hypotheses to explain drug binding to the channel, some believe that the guarded receptor hypothesis can be regarded as a mechanism for the change in affinity proposed by the modulated receptor hypothesis ${ }^{454}$.

The adverse arrhythmic effects are not restricted to AADs. A wide variety of currently used pharmacological agents have the potential to be proarrhythmic, including antibiotics xlviii such as Azithromycin ${ }^{460}$ and Clarithromycin ${ }^{461}, 462$; mood regulators such as SSRIsxlix463 and antipsychotic agents ${ }^{1464}$. These drugs have the propensity to prolong the QT interval and could lead in rare cases to fatal arrhythmias. Henceforth, improving our understanding of molecular and ionic mechanisms underlying arrhythmic activity will provide opportunities towards optimizing channel blocking profile and improving clinical efficiency and safety 465.

\subsection{Proarrhythmic Effects of Antiarrhythmic Drugs}

Flecainide acetate was released in the UK in 1983, preceded with several publications confirming its efficacy as an antiarrhythmic agent and its relative safety ${ }^{466,467}$. Initial observations with Flecainide

\footnotetext{
xlviThis can occur clinically during hyperkalemia for instance, which might worsen or decrease the action of some AADs. xlvii Refer to section 1.4.1 for a complete description of Nav1.5 conformational states.

xlviii Among the different classifications of antibiotics, macrolides have been widely investigated for their propensity to prolong the QT interval and subject patients to Torsades to Pointes (section 1.4.7), a lethal type of ventricular arrhythmias (Reference: J Pharmacol Exp Ther (1998) 286(2): 788-93).

xlix SSRIs are commonly known in the clinical practice and they stand for Selective Serotonin Reuptake Inhibitors.

${ }^{1}$ Antipsychotic agents belong to a class of psychiatric medications, also known as neuroleptics, primarily used to treat psychosis particularly in schizophrenia and bipolar disorders (Reference: Pharmacology. 4th Ed. (2009) Philadelphia: Lippincott Williams and Wilkins).
} 
have been confirmed on numerous occasions 468,469 . This drug was proven to be highly effective in the treatment of several types of arrhythmias ${ }^{468}$. Similarly to other AADs, Flecainide was reported to have side effects, but the majority was minor and generally well tolerated ${ }^{470}$. Guidelines recommended at the time that the drug be used with caution with patients suffering from severe $\mathrm{LV}$ dysfunction, due to the drug's negative inotropic effects, that would be detrimental if CV was further decreased in patients suffering from $\mathrm{HF}^{471}$. Although a truly controlled study about the frequency of proarrhythmic eventsli was not performed, but available comparative data suggested that Flecainide is no worse than any other AAD, when prescribed to patients with ventricular arrhythmias ${ }^{472}$. Other reports confirmed that a considerable number of patients, who suffered major life-threatening arrhythmia, were responding well to the treatment ${ }^{468}$. So why after 1989 , did Flecainide become a subject of controversy and intensive study? ${ }^{473}$

\subsubsection{CAST I: Increased Mortality Rate with Flecainide}

In an editorial discussing the unexpected and disappointing results of CAST I (1989) ${ }^{474}$, John Sanderson wrote 475: "In few specialties of medicine are new promising drugs shown to be so much inferior to placebo and, even worse, to increase mortality"476. CAST I or The Cardiac Arrhythmia Suppression Trial is a multicenter (in the USA and Canada), randomized, placebo-controlled study initiated in 1987, and designed to test whether suppression of symptomless or mildly symptomatic ventricular tachycardias occurring in the setting of myocardial infarction (MI)lii would also be associated with a reduction of sudden death ${ }^{473}$. Flecainide, among other AADs such as Encainide and Moricizine, were used in this study. The preliminary report of this study was published in 1989, and the trial was terminated when the results indicated that death from arrhythmia was 3-6 times more common in the treated group compared to placebo 474 . Flecainide and Encainide decreased the "number of nonlethal acute coronary events in CAST to an extent that corresponded to the increase in lethal events that the drugs produced"474. This means that in the setting of acute ischemia these Class Ic drugs caused lethal pro-arrhythmia by promoting V-Fib, transforming what would otherwise have been nonlethal events into cases of SCD by exacerbating ischemia-induced conduction delay ${ }^{465}$.

The outcomes of this large-scale clinical trial not only were unsatisfactory to investigators, but they were also to a great extent disconcerting and even, paradoxical. Since potentially fatal arrhythmias arise from ectopic stimuli that can alter the cellular synchrony in the heart leading to spontaneous oscillations in otherwise quiescent cells 300,477 , clinicians thought that depressing the tissue response to premature excitations should have antiarrhythmic properties 478 . CAST I showed that Flecainide, among other AADs that also decreased excitability and extended the refractory period in isolated cell studies ${ }^{479}$, ended up increasing the incidence of SCD in patients, despite successfully suppressing the cardiac response to at least $80 \%$ of PVCs 475 .

\subsubsection{Proposed Mechanisms of Flecainide Proarrhythmia}

The paradox behind AADs increased mortality profile in CAST I has been the focus of electrophysiological research for more than three decades so far. However, the ability of AADs to induce malignant tachyarrhythmias has been recognized much earlier than 1989, with the classic

\footnotetext{
li Velebit et al. chose the following criteria to define pro-arrhythmia: 1) a four-fold increase in the mean hourly frequency of ectopic beats compared with control period; 2) a ten-fold increase in the mean hourly frequency of ventricular couplets or ventricular tachycardia beats, or both compared to control period; 3 ) the first occurrence of sixty seconds or more of sustained V-Tach that was not present during the control period (Reference: Circulation (1982) 65: 886-894).

liiPatients recruited had had a myocardial infarction between 6 days and 2 years previously.
} 
report by Selzer and Wray on Quinidineliii-induced syncope in 1964480. Morganroth and Horowitz defined the term proarrhythmia to describe either the aggravation of an existing arrhythmia or the facilitation of new arrhythmias by means of AADs 481 . Proarrhythmia in the clinical setting can appear in a variety of forms, including increased frequency of PVCs, increased ventricular response to ectopic beats, facilitation of V-Tach induction and alterations in the properties of ventricular arrhythmia such that they show higher resistance to electrical cardioversion ${ }^{482}$.

Following CAST ${ }^{474}$, experiments were designed to test Flecainide in animals with prior MI $483,484$. Among these studies, were the ones that used epicardial mapping using extracellular electrodes to determine the mechanisms by which Flecainide promotes V-Fib in canine hearts with prior MI ${ }^{484-486 .}$ They concluded that preferential transversal conduction slowing or block in the infarcted zone facilitated the occurrence V-Tach. Flecainide was postulated to induce episodes of sustained V-Tach by slowing conduction in the ischemic zone enough to allow the electrical activity to reenter 485 . In these studies, Flecainide reduced the longitudinal and transversal velocity proportionately in the normal tissue and rarely induced V-Tach in animal hearts devoid of MI484, 485, 487. The prominent effects of Flecainide on conduction in the infarct zone without detectable effects on refractoriness set the basic mechanism for reentry and it became widely accepted that infarct size in these hearts was an important determinant of proarrhythmia484. As acute MI produces a variety of electrophysiological instabilities, which are not necessary found in a prior MI model 30,488 , additional studies focused on investigating the response of the cardiac tissue that had been exposed to Class Ic agents during an ongoing MI protocol ${ }^{489}$, 490. One study emphasized on the substantial drug concentration in the ischemic myocardial zone relatively to the remaining tissue, which was believed to be the cause behind excessively slowed conduction and block in that region ${ }^{489}$. Another study showed that the Class Ic agent amplified the ischemia-induced conduction delay in the myocardium, which in turn facilitated reentry in a large area of the tissue ${ }^{490}$. Carson et al. showed that another Class I blocker increased the propensity for V-Fib occurrence in the pig heart, following coronary artery ligation, with a rate dependence mechanism that favored $V$-Tach degeneration into a sustained form of $\mathrm{V}$-Fib ${ }^{491}$. These studies highlighted that the mechanism of Class I agents proarrhythmia is by promoting ischemic V-Fib secondary to their potent effects on cellular excitability and impulse propagation ${ }^{490-492}$. It was tempting to conclude, following extensive experimental literature review and the groundbreaking results of CAST I, that acute ischemia and infarction in the myocardium were the central factors that facilitated drug-associated mortality in large clinical trials such as CAST 493,494 , particularly when an analysis of non-fatal events in CAST suggested that Flecainide complicated what would otherwise have been non-lethal ${ }^{494}$.

In contrast to the experimental work done with Flecainide in the setting of myocardial ischemia and infarction, much less data were available regarding the proarrhythmic potential of Flecainide in the normal cardiac tissue or in the "structurally-normal" tissue ${ }^{482}$. Several hypothetical explanations regarding the adverse affects of AADs were postulated, after experimental data along with numerical simulations have been gathered to clarify how AADs might facilitate the initiation of potentially life-threatening arrhythmias in the normal myocardium. Nesterenko et al. showed that $\mathrm{Na}_{\mathrm{V}} 1.5$ blockers facilitate ventricular arrhythmia induction in isolated guinea pig hearts 495 , in consistency with their earlier published mathematical simulations describing the effects of $\mathrm{Na}_{v} 1.5$ blockade on cardiac vulnerability to arrhythmia ${ }^{492}$. Starmer et al. consequently hypothesized that slowing the recovery of $\mathrm{Na}_{v} 1.5$ availability might be associated with the amplification of proarrhythmic responses by increasing the time during which $\mathrm{Na}_{v} 1.5$ availability was spatially nonuniform, a time interval which they termed "vulnerability period" 475,496 . They also showed that

\footnotetext{
liiiAccording to the Vaughan-Williams AADs Classification, Quinidine is a Nav1.5 blocker agent belonging to Class Ia, possessing both conduction-slowing and APD prolongation actions (Reference: Cardiovasc Res (1983) 17:344-352).
} 
vulnerability of the cardiac tissue to premature stimuli was increased in the presence of usedependence block, a well-documented characteristic of $\mathrm{Na}_{\mathrm{V}} 1.5$ blockers (Class Ic) ${ }^{454,459}$.

Brugada et al. showed that Flecainide promoted V-Tach in nine out of ten thin sheets of Langendorff-perfused rabbit epicardium created after a transmural RV endocardial cryoablation, with no tachyarrhythmia being documented in any of the thin sheets prior to Flecainide exposure ${ }^{497}$. By mapping the epicardial surface using 248 extracellular electrodes, the tissue was spatially resolved to $2.25 \mathrm{~mm}$. They showed that Flecainide-induced tachyarrhythmia was based on reentry around an "arc of functional block", a mechanism that resembled the one proposed for proarrhythmia prior to MI, however without the involvement of a fixed anatomical obstacle ${ }^{497}$. Since no particular area was identified to be especially prone to conduction block, they hypothesized that Flecainide may have unmasked the natural spatial inhomogeneity in conduction properties, resulting in areas of long conduction delays ${ }^{497}$.

Another hypothesis was elegantly proposed by Antzelevitch et al., who suggested that spatial heterogeneity; an intrinsic property of the cardiac muscle (section 1.3.2), is responsible for the differential responsiveness of the ventricular myocardial tissue to Nav1.5 blocking ${ }^{498}$. Transmembrane potential measurements were carried out from the endocardium and the epicardium of isolated canine hearts exposed to Flecainide $[15 \mu \mathrm{M}]$, which revealed a contrasting response of these tissues to the drug, the reason for which was suggested to be in part due to a more prominent $\mathrm{I}_{\text {to }}$ in the epicardium than in the endocardium ${ }^{499}$. The resulting transmural dispersion of repolarization can form regions that block propagating wave, creating therefore a robust substrate for reentry 262 (section 1.4.4).

\subsection{This Thesis}

Sudden death often follows cardiac rhythm disturbances that are initiated by excitation of electrically unstable tissue ${ }^{496}$. The work presented in this thesis is centered on characterizing macroscopic electrophysiological instabilities observed at the tissue level that emanate from perturbations affecting essentially the $\mathrm{Na}_{\mathrm{v}} \mathrm{1.5}$ channel in the murine heart. We tried to approach this question by directly targeting $\mathrm{Na}_{v} 1.5$, either through genetically modified mouse models that mirror fatal pathologies observed in man, or through the use of antiarrhythmic drugs that principally modify the channel's function in its respective environment. The hallmark of an unstable tissue is that excitability is spatially heterogeneous ${ }^{475}$; hence characterizing these instabilities in excitability and conduction will require a technical tool that provides the spatialtemporal resolution necessary to map the electrical activity from the epicardial surface of the invitro perfused heart. Optical mapping has provided us with the capacity to measure the propagation of the electrical wave on the surface of the intact heart, with a spatial resolution down to a few epicardial cardiac cells in one pixel and a temporal resolution more than ten times faster than the time it takes the actual electrical wave to travel across the epicardium. Using this technique, our aim was to measure more accurately a set of biophysical parameters and to elucidate the fundamental relationships between them in distinct cardiac substrates and within different pharmacological protocols, integrating knowledge and data from the molecular ionic level to the whole organ.

To fulfill this aim, we investigated two genetically modified mouse models: the $m d x$-mouse model of Duchenne muscular dystrophy (section 1.2.2) and the $\triangle$ KPQ mouse model of LQTS3 (section 1.4.3), each compared to their respective WT control. The pathophysiology of each of these fatal diseases has been described in their respective sections. We adopted a generic approach to these models, by focusing on one denominator: the Nav1.5 channel and its modulation by antiarrhythmic drugs (Flecainide, section 1.5). Although, we appreciate the entanglement of the molecular pathways 
underlying the different pathologies, we aimed in our optical mapping investigations at describing the translational axis that connects the ion channel on one side to macroscopic electrical instabilities on the other side. We adopted a generic approach to fulfill this goal, by subdividing the study over three phases of investigation, as follows:

\subsubsection{Conduction Abnormalities in the mdx-mouse: A Model of Lower NaV1.5 Availability}

The monogenetic disease investigated is caused by a mutation in the gene encoding the large cytoskeletal protein, dystrophin ${ }^{500}$ (section 1.2.2). A recent study showed that dystrophin regulates the cardiac sodium channel ${ }^{238}, \mathrm{Na}_{\mathrm{V}} 1.5$ (section 1.4 ) on the LM of the cardiomyocyte. Nav1.5 mediates the fast inward sodium current (section 1.1.4) that initiates electrical excitation in most cardiac cells (section 1.3.6). The absence of dystrophin is associated with a non-uniform loss of Nav1.5 from the plasma membrane of the cardiomyocyte ${ }^{238}$. Consequently, we wanted to investigate whether this molecular finding had any repercussions on the conduction velocity in the medium, and whether conduction abnormalities can be detected by optically mapping the intact $m d x$ mouse heart (the mouse model harboring dystrophin deficiency, section1.2.2). Conduction measurements were carried out to characterize potential propagation instabilities that could ensue in a cardiac substrate of lower $\mathrm{Na}_{\mathrm{v}} 1.5$ availability (section 3.1).

\subsubsection{Implementation and Validation of Analytical Strategies in CV Evaluation in the Murine Heart Using Optical Mapping}

The use of murine hearts for gathering electrophysiological data is a challenging task. It's faced with technical difficulties that start with the rapid heart rate 501 , the undersized heart, the presence of heterogeneities ${ }^{44,502}$ at both the cellular and structural levels ${ }^{503}$ (section 1.3.2), and the restricted time for the spread of any activating wave front ${ }^{504}$. The discrepancies found in the electrophysiological data justify the use of high spatial-temporal resolution techniques, such as optical mapping, to reassess and reevaluate CV measurements with higher accuracy284. Optical mapping of intact hearts using fluorescent voltage reporter dyes is a powerful, well-established and widely used technique to study electrical patterns occurring in the cardiac tissue 505 . The precise quantification of CV for the same cardiac substrate is often controversial284, 506, 507. Several methods have been implemented in the study of CV in mouse hearts under simple 238,508 and more complex activation patterns 509,510 . While they are appearing more and more frequently in publications, their implicit assumptions, errors and limitations are rarely subject of overt debate. From epicardial AP spreads, we obtained information on action potential (AP) generation, electrophysiological properties of the cardiac substrate via patterns of activation and the apparent CV in the medium of propagation. We implemented three different strategies to our experimental data (section 2.2.4), and compared the outcomes of each method and validated the results under anisotropic and nonanisotropic conditions (section 3.1.4). We challenged each method with synthetic data emphasizing on the effects due to noise (section 3.1.6) and heterogeneity in propagation (section 3.1.7).

\subsubsection{Characterization of Spatial-Temporal Heterogeneity Induced by Flecainide Using Clinically Valid Concentrations}

Scientists' growing suspicion regarding the predictive capacity of single cell measurements was triggered by the counterintuitive results of CAST I in 1989474. Despite the fact that the antiarrhythmic activity of $\mathrm{Na}_{1} 1.5$-blockers has been demonstrated quite robustly and redundantly 458, 511-513 over more than three decades of experiments, the increased mortality rates of CAST I disproved this predictive potential. Flecainide, being an antiarrhythmic drug known for its strong modulation of $\mathrm{Na}_{\mathrm{V}} 1.5^{466}$, has been shown to evoke proarrhythmic responses in cardiac 
tissues exposed to the drug474, 482. Since then, several hypotheses have been proposed to clarify the mechanisms behind the electrical instabilities that collectively develop in the intact tissue 495, 497, 499 . We investigated the effects of this drug using clinically valid concentrations, to observe and measure the different biophysical parameters at play that could give us some insights on the electrical instabilities emanating from modifying the channel in three different cardiac substrates, with a gradient in Nav1.5 functionality: one with lower Nav1.5 availability ( $m d x$, section 3.2 ), the second one with a normally functioning channel (WT, section 3.2.2) and the last one with a hyper-functional channel $(\triangle \mathrm{KPQ}$, section 3.3). In this regard, optical mapping is a fundamental experimental tool in the study of spatially extended electrophysiological heterogeneity of the cardiac substrate, without which appreciation of the complexity of these models wouldn't have been possible. 
(This page is intentionally left blank) 



\section{Chapter 2}

\section{Experimental Methods}

The present chapter details the methods used to obtain the experimental results of this thesis. The main experimental protocol used is optical mapping of the propagating electrical wave on the surface of the murine left ventricular (LV) free wall epicardium, using Di-4-Anepps, a voltage sensitive dye (VSD). Section 2.1 outlines the details the optical mapping setup in terms of hardware and software used: vECG recordings and pacing electrodes (2.1.1), the details of murine heart isolation (2.1.2). Optical mapping of membrane voltage measurements were performed using an electromechanical decoupler and a VSD (2.1.3) and details of the setup are elaborated in section 2.1.4. Section 2.2 outlines the details concerning the optimization of signal quality for further analysis and the mathematical background of the strategies used in this thesis to evaluate the CV in the medium of propagation. Starting with processing the raw optical signal and the creation of activation maps (2.2.1), to dispersion maps (2.2.3), we also show the methodological approaches in the evaluation of CV 2.2.4, as well as the numerical data used to simulate propagation in the murine heart, which are detailed in section 2.2.5. Results of the optical mapping data are presented in Chapter 3 and are further discussed in Chapter 4.

The study of electrophysiological properties of the healthy and diseased heart provides important insights into the understanding of the complex electrical activity, which requires the development of mapping techniques that simultaneously record spatial and temporal information. Traditionally, surface electrodes have been, and continue to be, used measure extracellular cardiac potentials ${ }^{514}$ (Figure 5b). However, this type of surface mapping actually suffers from several drawbacks, such as low spatial resolution and reduced flexibility (since only a finite number of electrodes can be placed on the surface of one heart with a fixed spacing between electrodes once the electrode array is set), low depth of field and electrical (interference) artifacts from stimulating electrodes ${ }^{514}$. The mouse heart is a widespread model for cardiovascular studies, due to factors like the existence of low cost technology for genetic engineering in this species ${ }^{515}$, the relatively fast reproduction capacity of the animal and the ease of handling the animal for experimental work. Nevertheless, the use of murine hearts for gathering electrophysiological data is a particular challenging task. It's faced with several technical difficulties that start with the rapid heart rate ${ }^{501}$, the undersized heart (the apico-basal distance is $\sim 6 \mathrm{~mm}$ ) and the restricted time for the spread of any activating wave front (a propagating electrical wave can traverse the entire epicardium in less than 6-8ms) ${ }^{504}$. For such a small heart, the number of extracellular electrodes is limited by spatial constraints and a lower number of detection sites makes the electrode array a poor choice for detecting complex electrical patterns.

Optical mapping with VSDs has made it possible to record cardiac APs with high spatial-temporal resolution that is otherwise not attainable using electrode arrays 505 . Optical techniques use changes in transmitted light from the prep to map the electrical activity. VSDs are compounds that bind to cell membranes and fluoresce with an intensity proportional to the local membrane potential ${ }^{516}$, 
and with a response time which is several orders of magnitude faster than the most rapid changes in the cardiac membrane potential517. VSDs can produce phototoxic effects. For instance, Di-4-Anepps, a widespread used VSD in cardiac mapping, is reported not to be toxic at low concentrations in the absence of light, but degrades membranes in the presence of increased levels of light, where light absorption at high intensities can cause tissue heating and alterations in electrophysiological parameters ${ }^{518}$. Therefore, a major advantage of electrode arrays is that the prep is not subjected to potentially phototoxic effects of potentiometric dyes ${ }^{519}$. In addition, these electrodes record are very fast and capable of measuring the AP upstroke at a temporal rate close to $0.1 \mathrm{~ms}^{520}$, 521. Another fundamental difference between electrodes and optical AP (OAP) recordings is related to the source of these signals ${ }^{516}$. Using extracellular electrodes, the source is a single cell, whereas an OAP originates from a small lump of cells, where the overall volume depends on several factors such as optical magnification, detector size, light transmission properties of the cardiac tissue ${ }^{516}$. For comprehensive reviews regarding optical mapping, refer to the reviews by Girouard et al.516 (1996), Efimov $^{505}$ (2004), Herron and Jalife 514 (2012).

\subsection{Setup and Tissue Preparation}

Nearly all mapping systems consist of a number of sensitive detectors, from which the signal gets amplified, processed, displayed and then stored for further analysis.

\subsection{1. vECG and Pacing Electrodes}

vECG recordings associated with spontaneous sinus and paced rhythms, were obtained steadily throughout the experiment and stored for off-line analysis (Figure 5b). Signals were amplified and low-pass filtered at $200 \mathrm{~Hz}$ using an electronic amplifier (HSE, Germany) and digitally sampled at 10 $\mathrm{kHz}$ with a 16-bit resolution using the NI USB-6216 BNC (National Instruments) digitizer and custom-made software using LabView (National Instruments). At the center of the left ventricular wall, a bipolar specialized cardiac pacing electrode (50-100 kOhm, FHC, USA) was gently placed, stimulating the preparations at a BCL of $100 \mathrm{~ms}$ at $2.5 \times$ diastolic threshold, with $2 \mathrm{~ms}$ impulse duration. Capture was confirmed both electronically and optically.

\subsubsection{Murine Heart Isolation and Langendorff Perfusion}

Mice were heparinized (40 units) and anesthetized using volatile 100\% Isoflurane (Floren, AbbatGermany). Excised through a sternotomy, intact hearts were carefully cannulated, connected to a custom-made perfusion setup and retrogradely perfused at 2.5-3 mL.min $^{-1}$ via the aorta with Tyrode solution, under quasiphysiological conditions. The temperature was set to $38 \pm 1^{\circ} \mathrm{C}$, oxygen content $\left(95 \% \mathrm{O}_{2}\right.$ and $\left.95 \% \mathrm{CO}_{2}\right)$, pH to 7.4 and ionic concentration (in mmol.L-1): $\mathrm{NaCl} 130, \mathrm{NaHCO}_{3} 24, \mathrm{KCl} 4$, $\mathrm{MgCl}_{2} 1, \mathrm{CaCl}_{2} 1.8, \mathrm{KH}_{2} \mathrm{PO}_{4}$ 1.2, $\mathrm{C}_{6} \mathrm{H}_{12} \mathrm{O}_{6}$ 5.6, 1\% albumin (Bovine Serum Albumin, Sigma) and insulin (Insuman Rapid, Sanofi-Aventis, 5IU/L). Hearts were immersed with constantly warm Tyrode solution, within a custom-made glass chamber that ensures adequate temperature throughout the experimental period, where the solution within serves as a conducting medium for in-vitro custommade Ag-AgCl ECG electrodes placed horizontally and parallel to the septum, at a distance of $1 \mathrm{~mm}$ from the epicardial surface. Hearts with a spontaneous beating rate exceeding $450 \mathrm{beats} / \mathrm{min}$ for more than $20 \mathrm{~min}$ after connecting them to the perfusion setup were exclusively used for further measurements. The left ventricular free wall was projected with maximal cross-sectional diameter in the objective light path to ensure consistent mapping of the conduction spread, by maximizing the exposed stimulated area to the exciting monochromatic beam (Figure 5a). 
a
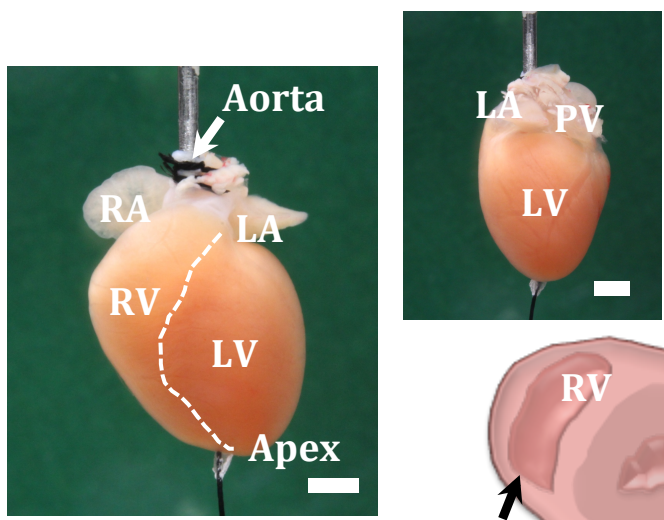

Epicardium

Endocardium

CMOS

(2kHz, 100×100pixels)
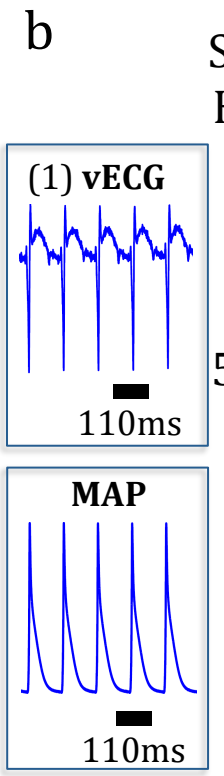

Short-arc Hg Lamp
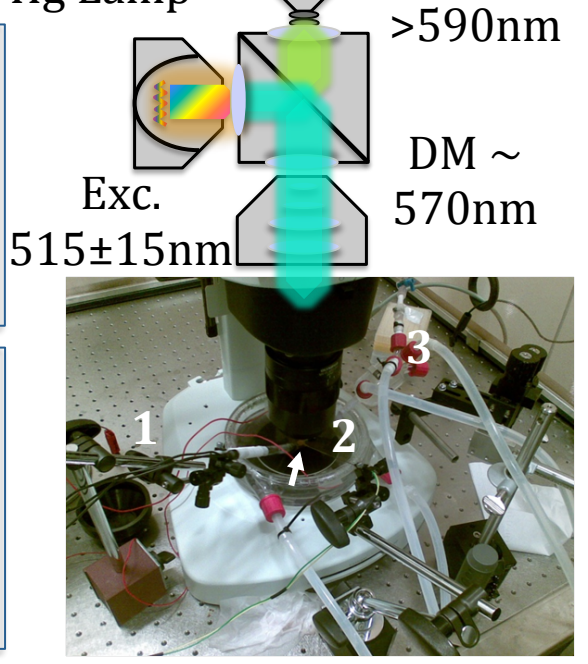

$\Delta \mathbf{F}$ (FC 5\%)

$\mathrm{DM} \sim$ $570 \mathrm{~nm}$

Emis.

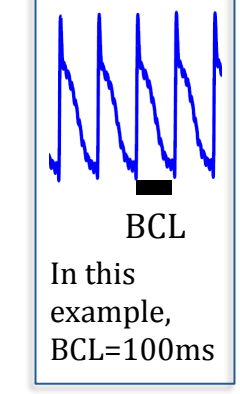
$>590 \mathrm{~nm}$

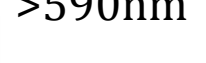

(2) Pacing $1.5 \mathrm{~ms}$

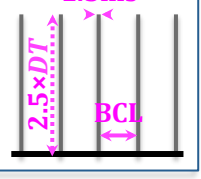

Figure 5. Experimental preparation and signal samples recorded during LV free wall pacing of the murine heart. a. External anatomy of murine heart and cross section (cartoon) delineated. An intact murine heart cannulated and installed in a Langendorff retrograde perfusion. Dotted line marks the borders between $\mathrm{RV}$ and LV. The distance endo-epic is $2.2 \mathrm{~mm}$ and the epicardial layer estimated $\sim 0.3-0.5 \mathrm{~mm}$ deep. In cross section, the bulky LV is of considerable volume compared to RV. Annotations on figure: $\mathbf{R A}=$ Right Atrium; $\mathbf{L A}=$ Left Atrium, $\mathbf{P V}=$ Pulmonary Veins; Horizontal Scale Bar $=1 \mathrm{~mm}$. (Picture credit to: Dura, M. and SchröderSchetelig, J.). b. Hardware and example signals. The light source is a short arc mercury lamp. The light passes through a shutter and focused on filter cube that homogenizes the beam into a green monochromatic light of $515 \pm 15 \mathrm{~nm}$. The emitted fluorescence is passed through a dichroic mirror (DM) of $570 \mathrm{~nm}$, which is further long passed filtered $(>590 \mathrm{~nm})$. The light is projected on a CMOS chip of 100 by 100 pixels, and the measurements are temporally sampled at $2 \mathrm{kHz}$. Left: (above) vECG showing the normal sinus rhythm (here $\sim 9 \mathrm{~Hz}$ ); (below) Monophasic AP recording (sinus rhythm corresponding to above vECG signal). Right: (Above) OAP, with fractional change of $5 \%$, paced at $10 \mathrm{~Hz}$. (Below) Steady state pacing protocol, pulse duration $=1.5 \mathrm{~ms}$, at 2.5 times diastolic threshold. Arrow: heart in perfusion, 1: vECG electrodes, 2: pacing electrode, 3: perfusion chamber.

\subsubsection{Excitation-Contraction Decoupler and VSD Staining}

Of the limitations of cardiac optical mapping of the heart are imaging artifacts, caused by successive contractions. Motion artifacts can strongly distort or obscure voltage optical measurements. Several strategies are adopted to overcome this obstacle, which could be mechanical in nature, pharmacological or numerical. We adopted a pharmacological one by using an electromechanical decoupler $\left(5 \mu \mathrm{M}\right.$, Blebbistatin, Sigma), which acts basically as a specific myosin II inhibitor ${ }^{222}$, but does not have any noticeable effects on cardiac electrophysiology under these experimental conditions ${ }^{523}$.

Fluorescence dye can be divided into slow and fast acting dyes. Slow dyes change their locations with cells (i.e. move between intracellular and extracellular compartments) in response to membrane potential changes with very slow kinetics (seconds to minutes) ${ }^{524}$. These dyes, with such 
Figure 6. Steady-state pacing conditions (10Hz). a. Optical mapping signal showing a sequence of paced APs. The normalized signal is shown here, with a SNR 20 and FC 5\%. The red dotted line indicates the consecutive pulses to be temporally averaged in order to reduce beat-to-beat variability. In our measurements, 10 pulses are usually averaged under steady state conditions (here only 6 are shown). b. Standard deviation (STD) map showing the standard deviation at each pixel. A white line delineates a mask on the LV free wall. Since all averaged AP are similar in pixels covering the $\mathrm{LV}$, the STD is near 0 . At $10 \mathrm{~Hz}$, the atria entrained by the sinus rhythm are out of phase with the ventricles, so the STD is high. Scale bar $=1 \mathrm{~mm}$. c. Curves showing the change in APD at different numbers of pulses (WT,

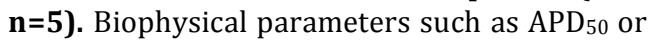
$\mathrm{APD}_{90}$ vary progressively with entrainment from sinus rhythm. The curves show APD 50 and APD90 increasing gradually to reach steady state values at 20 pulses, beyond which additional pulses didn't affect the values significantly, indicating steady state conditions (these curves were made with WT hearts. The same procedure was repeated for $m d x$ hearts). d. Differential of the curves shown in (c) over number of pulses. The curves cross $y=0$ (dotted black line) when 20 pulses are given consecutively to entrain the heart at $10 \mathrm{~Hz}$. Additional pulses don't change the value of the parameter measured. From these experiments, we concluded that 20 pulses should be given before optical measuring starts.

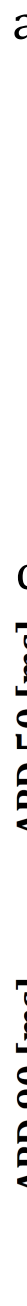

$\mathrm{b}$
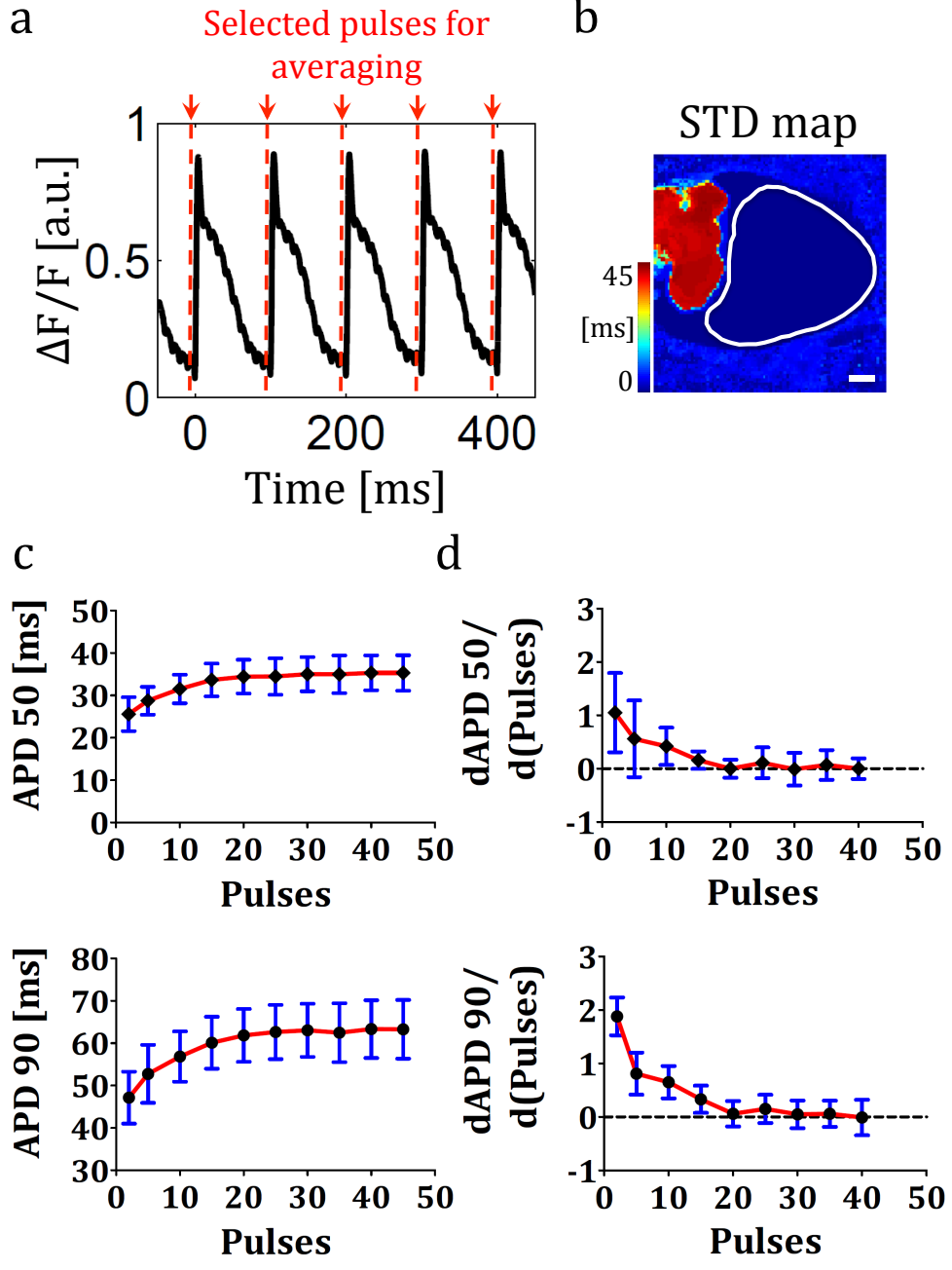

large time constants, are not suitable for mapping fast activities like the ones taking place in the murine heart, where the entire ventricular AP starts and terminates in $\sim 40 \mathrm{~ms}$.

Fast dyes, on the other hand, do not rely on movement back and forth between the different compartments of the cardiac cell, but change their optical characteristics by changing conformation. These dyes provide rapid absorbance and fluorescence responses to membrane potential (time constants in the order of one $\mathrm{ms}$ ) and are capable of recording fast $\mathrm{AP}^{525}$. Fast dyes are divided into different categories based on their chemistry. Several mechanisms account for a given dye's sensitivity, including changing its conformation, its orientation within the plasma membrane or its charge distribution (electrochromism) within the plasma membrane ${ }^{526}$. The rotation-dimer mechanism has been suggested as a variant of molecular motion, by which the dye switches with a change in voltage from a monomeric form, which is basically oriented perpendicular to the membrane, to a dimer oriented parallel to the membrane ${ }^{526}$. Electrochromism, another mechanism explaining the function of VSD, refers to the direct displacement of charges within a molecule by external electric fields 527 . As there is no change in the molecular arrangement, electrochromic processes take place at the nanosecond time scale527. Loew et al. have provided evidence that Di-4ANEPPS (the VSD used in our experiments ${ }^{\text {liv, }}$, stock solution $1.25 \mathrm{mg} / \mathrm{mL}$, aliquots of $100 \mu \mathrm{L}$ ) respond

liv In the mouse heart, the endo-epicardium distance was measured to be around $2.2 \mathrm{~mm}$ (this value is congruent with the value reported by Costa et al. Am J Physiol Heart Circ Physiol (2012) 302(1): H270-277). Based on the work of Bishop et al. 
to membrane voltage changes by an electrochromic mechanism, i.e. by charge redistribution of the surface of the molecule527. For a comprehensive review on organic dyes, their chemistry, mechanisms and sensitivity in optical mapping, refer to the book chapter by Loew "Design and Use of Organic Voltage Sensitive Dyes" (2010) ${ }^{526}$.

\subsubsection{D Optical Mapping Setup}

After the heart rate reached steady state (typically $20 \mathrm{~min}$ from initiation of retrograde perfusion), measurements were carried out after staining the heart with a voltage-reporter dye (Di-4-ANEPPS, Molecular Probes, Portland, OR), by injecting a $1 \mathrm{~mL}$ bolus at $30 \mu \mathrm{mol} . \mathrm{L}^{-1}$, into a $10 \mathrm{~mL}$ compliance chamber proximal to the aorta (section 2.1.2). Concisely, excitation light from a 100W short-arc mercury lamp (HB0103W/2, Olympus, Germany) was collocated, using an upright macroscope (MVX10, Olympus) and made monochromatic using an interference filter $(515 \pm 15 \mathrm{~nm})$ and dichromatic filter $(570 \mathrm{~nm})$, then collected using a long-pass filter $(>590 \mathrm{~nm})$ and projected onto a $100 \times 100$ pixel CMOS (Complementary Metal Oxide Semiconductor camera, Ultima-L, SciMedia) recording array (Figure 5b). Imaging with Di-4-ANEPPS was obtained at a frame rate of $2 \mathrm{kHz}$. To reveal the signal, background noise was subtracted from each frame. Imaging was carried out using a $0.63 \times$ objective (MVPLAPO $0.63 \mathrm{X}$, NA 0.15 ; Olympus), at a zoom of $2.5 \times$ in order to provide the largest view of the left ventricular free wall with a pixel resolution of $100 \mu \mathrm{m}$. Prior to any further analysis, the optical signal was modified in order to optimize the fluorescence change and increase the SNR. To study conduction spread at steady state pacing of $10 \mathrm{~Hz}$, preparations were paced using a bipolar electrode (Figure 5b, section2.1.1). The raw data collected from the camera were frameselected using custom-made open source software written in Java that was optimized for the mouse heart, constituting a platform input into another custom-made routine written in MATLAB (the Mathworks) for further analysis.

\subsubsection{Animal Models Used in the Current Study and Drugs}

Adult 12-16weeks male dystrophin-deficient ${ }^{l v}$ B6Ros.CG-Dmd $(m d x-5 \mathrm{cv}) / \mathrm{J}$ micelvi $\left(\mathrm{n}^{\mathrm{lvii}}=17+7\right)$ along with their WT gender and age matched controls C57BL6/J $(n=17+10)$; and 20-22 weeks old male heterozygous $\triangle K P Q$ micelviii $(n=3+4)$ with their corresponding WT control $(n=3+4)$ were selected for the following studies. To avoid any position induced changes in fiber orientation, to maximally expose the region of fluorescence and to provide a supposedly homogeneous area of conduction spread; we restricted the measurements to the LV free wall for all excised hearts. Hearts were stained with a Di-4-ANEPPS and activations were recorded as waves emanated from the tip of a bipolar electrode, placed at the centre of the LV free wall, under automated steady state pacing at $B C L=100 \mathrm{~ms}$. The pharmacological intervention consisted of perfusing the cannulated hearts with the regular Tyrode solution (section 2.1.2) containing $1 \mu \mathrm{M}$ Flecainide (Flecainide Acetate, Sigma Aldrich) after the control (non-treated) measurement was recorded and showed an elliptical behavior. Measurements with Flecainide were taken at time points 0, 1, 3, 5, 7 and 10 min, to assure close monitoring of activation changes due to the drug. Unlike the experimental protocol used for the $m d x$ and their WT counterpart that lasted up to $10 \mathrm{~min}$ exposure to Flecainide, steady state was

(Biophys J (2006) 90:2938-2945), fluorescence in rabbit hearts loaded with Di-4-Anepps is optimal between 0.5-1mm below the epicardial surface.

lv Dystrophin-deficiency is described in the Introduction section 1.2.2.

lvi These mice will be referred to as $m d x$ (for simplicity) throughout this work.

lvii $\mathrm{n}=$ number of experiments. Two major experimental studies were conducted in this thesis. $\mathrm{n}=\mathrm{x}+\mathrm{y}$ means the total number of animals used for both studies, where $\mathrm{x}$ indicates the number of animals used in the study that investigated the different analytical strategies in CV evaluation using optical mapping, and y indicates the number of animals used to conduct the heterogeneity study with Flecainide.

lviii The $\triangle \mathrm{KPQ}$ mouse, harboring the mutation for Long QT syndrome 3, is described in the Introduction section 1.4.3. 
no longer achievable for the $\triangle \mathrm{KPQ}$ beyond $5 \mathrm{~min}$ of treatment (i.e. either entrainment at $10 \mathrm{~Hz}$ frequency was no longer possible or consecutive APs showed considerable beat-to-beat variability which prevented temporal averaging). All animal procedures were reviewed and approved by the Institutional Animal Care and Use Committee of the University Medical Center Göttingen and by veterinarian state authority LAVES (Niedersächsisches Landesamt für Verbraucherschutz und Lebensmittelsicherheit) in compliance with the humane care and use of laboratory animals.

\subsection{Analyzing the Optical Signallix}

\subsubsection{Signal Processing}

The image sequence was filtered spatially using a $5 \times 5$ uniform kernel. At each pixel, the time series was filtered in time using a $5 \times 5$ uniform kernel, baseline subtracted, normalized to with respect to its peak amplitude to [0 1 ] intensity values and inverted (Figure 6a). For the paced recordings only, the optical signal was further averaged over 10 activations to improve SNR and remove infinitesimal beat-to-beat variability (Figure 6a). The cardiac excitation propagates as a moving wave front, segregating the depolarized tissue from the quiescent tissue; consequently boundaries at $0.5 \mathrm{~ms}$ interval (corresponding to the sampling rate) were computed by a linear interpolation of activated pixels (Figure 7), as the wave front propagates anisotropically from the tip of the stimulating electrode (Figure $5 b$ ).

Signal Analysis. In this section, the analysis of the optical recordings will be described in detail. It is based on the recorded optical signal $s(x, y, t)$. Note that, in fact, only the set of values

$$
\left\{s\left(x_{i}, y_{j}, t_{k}\right) \mid 1 \leq i \leq N, 1 \leq j \leq M, 1 \leq k \leq K\right\}
$$

is known. $\left(x_{i}, y_{j}\right)$ denotes the position of the $i^{\text {th }}$ out of $N$ pixels in the x-direction and the $j^{\text {th }}$ out of $M$ pixels in the $y$-direction and $t_{k}$ is the time at which the $k^{\text {th }}$ out of $K$ frames was recorded, i.e.

$$
\begin{aligned}
& x_{i}=i \cdot \Delta x \\
& y_{j}=j \cdot \Delta x \\
& t_{k}=k \cdot \Delta t
\end{aligned}
$$

where $\Delta x$ and $\Delta t$ are the space and time resolution of the cameras. Whenever physical units are not required, we will refer to the value $s\left(x_{i}, y_{j}, t_{k}\right)$ simply as $s(i, j, k)$. Before performing any signal analysis, the optical signal is averaged using a box filter of 5 pixels by 5 pixels in space and 5 samples in time.

\subsubsection{Activation Maps}

An activation map $a(x, y)$ indicates the time of the first activation of the tissue at position $(x, y)$ after some reference time $t_{r e f}$. The reference time usually corresponds to the time of a stimulus in order summarize the wave activity emerging from the stimulation site. For simplicity, we assume that the data only contain the response to one stimulus and hence $t_{r e f}=t_{1}=0$. For all $i$ and $j$, we define the normalized signal $\tilde{s}(x, y, t)$ as:

$$
\tilde{s}(i, j, k)=\frac{s(i, j, k)-\min _{1 \leq k^{\prime} \leq K}\left[s\left(i, j, k^{\prime}\right)\right]}{\max _{1 \leq k^{\prime} \leq K}\left[s\left(i, j, k^{\prime}\right)\right]-\min _{1 \leq k^{\prime} \leq K}\left[s\left(i, j, k^{\prime}\right)\right]}
$$

lix This section is taken from a manuscript in preparation with other co-authors: Raad, N., Bittihn, P., Lehnart, S.E., and Luther S. 
The activation time can be thought of as the time point at which the excitation wave front passes through the myocardial cell. Since the originally recorded optical signal is inverted, we choose a threshold $\hat{s}$, below which the tissue should be regarded at activated. To account for noise, we only accept a drop of the signal below threshold as an activation, if it is followed by $\tilde{k}$ consecutive frames that are, on average below threshold. Thus for all pixels $(i, j)$, the frame of activation $k_{a c t}(i, j)$ is defined as

$$
k_{a c t}(i, j)=\min \left(\left\{k \mid 1 \leq k \leq K \wedge \tilde{s}(i, j, k-1) \geq \hat{s} \wedge \tilde{s}(i, j, k)<\hat{s} \wedge\left\langle s\left(i, j, k^{\prime}\right)\right\rangle_{k \leq k^{\prime} \leq k+\tilde{k}}<\hat{s}\right\}\right)
$$

In the examples shown in this paper, we use $\hat{s}=0.5$ (Figure 7a) and $\tilde{k}=10$ (Figure 7a). To get a subframe estimate of the time of activation, we linearly interpolate $\hat{s}$ between $k_{a c t}(i, j)-1$ and $k_{a c t}(i, j)$, which leads to the definition

$$
a(i, j)=t_{k_{a c t}(i, j)}+\Delta t \cdot\left(\frac{\hat{s}-\tilde{s}\left(i, j, k_{a c t}(i, j)-1\right)}{\tilde{s}\left(i, j, k_{a c t}(i, j)\right)-\tilde{s}\left(i, j, k_{a c t}(i, j)-1\right)}\right)
$$

for each pixel $(i, j)$ of the activation map. To reduce noise artifacts, activation maps for 10 pulses of the same stimulation sequence are averaged (Figure 6a). Beat-to-beat variability can be monitored using pixel-wise standard deviations (Figure 6b).

a

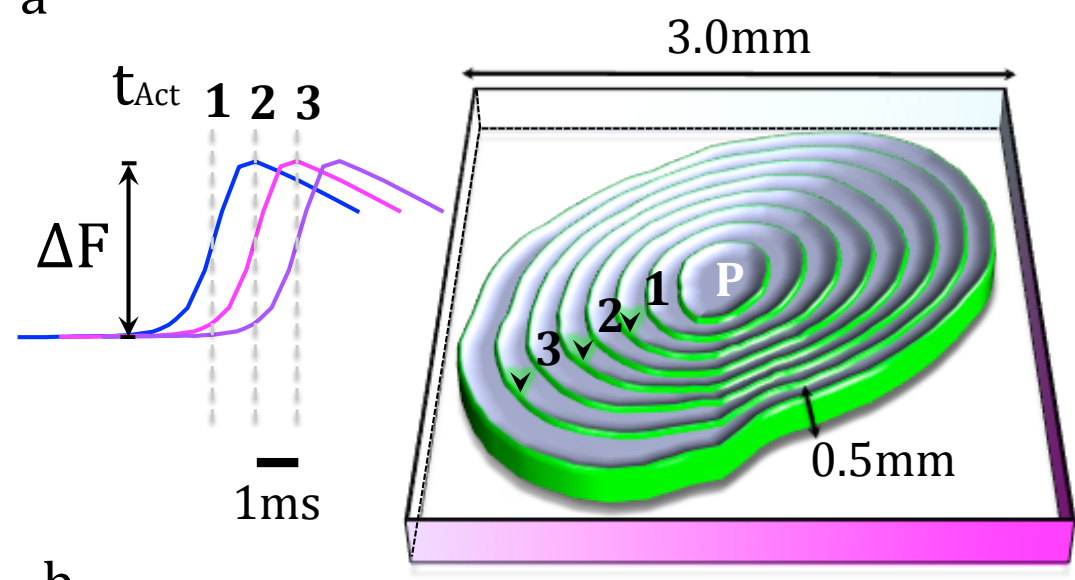

b

WT

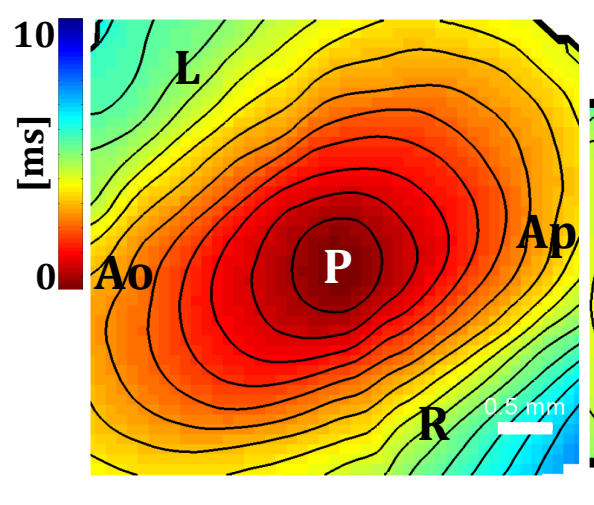

mdx

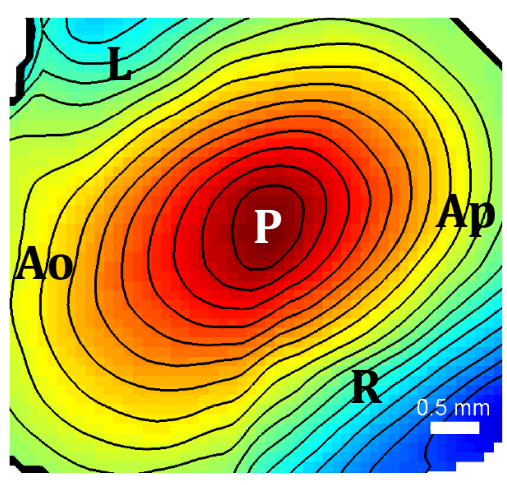

Figure 7. Activation times and creation of activation maps for WT and $m d x$. a. 3D scheme of the wave propagation on the epicardial surface (depth $0.5 \mathrm{~mm}$ ) from a point stimulation $(P)$. The numbers $(1,2,3)$ of the AP upstrokes on the left represents the activity in the corresponding points on the surface (right). Activation times were detected whenever the upstroke crossed the threshold at $50 \%$ fluorescence $(\Delta \mathrm{F})$ for more than 10 consecutive frames. Interpolation of the different pixels with the same activation time formed an isochronal line. The points (1, 2 and 3 ) are separated by 2 isochrones, i.e. a temporal interval of $1 \mathrm{~ms}$ (temporal resolution $=0.5 \mathrm{~ms}$ ). b. Activation maps recorded from the epicardium of WT (left) and $m d x$ (right) hearts. As the wave propagates from a point stimulation, a snapshot each $0.5 \mathrm{~ms}$ was taken. The activated pixels formed an ellipse that gradually increased in size and the wave reached the boundaries, which delineated the typical anisotropic propagation on the epicardium. Annotations in figure: $\mathbf{P}=$ Pacing, $\mathbf{A p}=$ Apex, $\mathbf{A o}=$ Aorta, $\mathbf{L}=$ Left, $\mathbf{R}=$ Right. Scale bar $=0.5 \mathrm{~mm}$. 


\subsubsection{ROI and Dispersion Maps}

Further analysis was based activation maps $a(x, y)$ obtained as described in the previous section. Within this map, we define a region of interest (ROI), as a subset of pixels that satisfies the following criteria (Figure 8b):

- A homogeneous fluorescence intensity field where individual pixels can be evaluated as a whole and are assumed to share fairly similar physiological variables ${ }^{528}$;

- Exclusion of stimulation artifacts projected in the earliest contours as well as boundary or curvature artifacts in the last contours that are well known sources of inhomogeneities ${ }^{505}$, 529,530 .

Figure 8. APD duration and upstroke velocity $(\mathrm{dF} / \mathrm{dt})_{\max }$ analysis using optical AP recordings with their associated APD and $(\mathrm{dF} / \mathrm{dt})_{\max }$ dispersion maps. $a$. Determining APD and (dF/dt) max using optical AP. APD 50 is determined by the time interval from the time point of the takeoff potential (arrow with $0 \mathrm{~ms}$ ) till the normalized AP repolarizes back to 50\% from the peak. The amplitude between takeoff and peak represents $100 \%$ (which is similar to the $100 \%$ repolarization from peak to resting membrane potential). Red dotted lines (upper 10\% and lower 10\% thresholds) cross the AP upstroke at two locations, from which $(\mathrm{dF} / \mathrm{dt})_{\max }$ can be calculated (amplitude in a.u. over time in s). b. ROI between isochrones 2 and 6 . Blue and red arrows represent propagation along longitudinal and transversal directions (APs at these locations are picked up along these axes for further analysis). c. APD and $(\mathrm{dF} / \mathrm{dt})_{\max }$ dispersion maps showing the gradually changing spatial properties of these parameters.

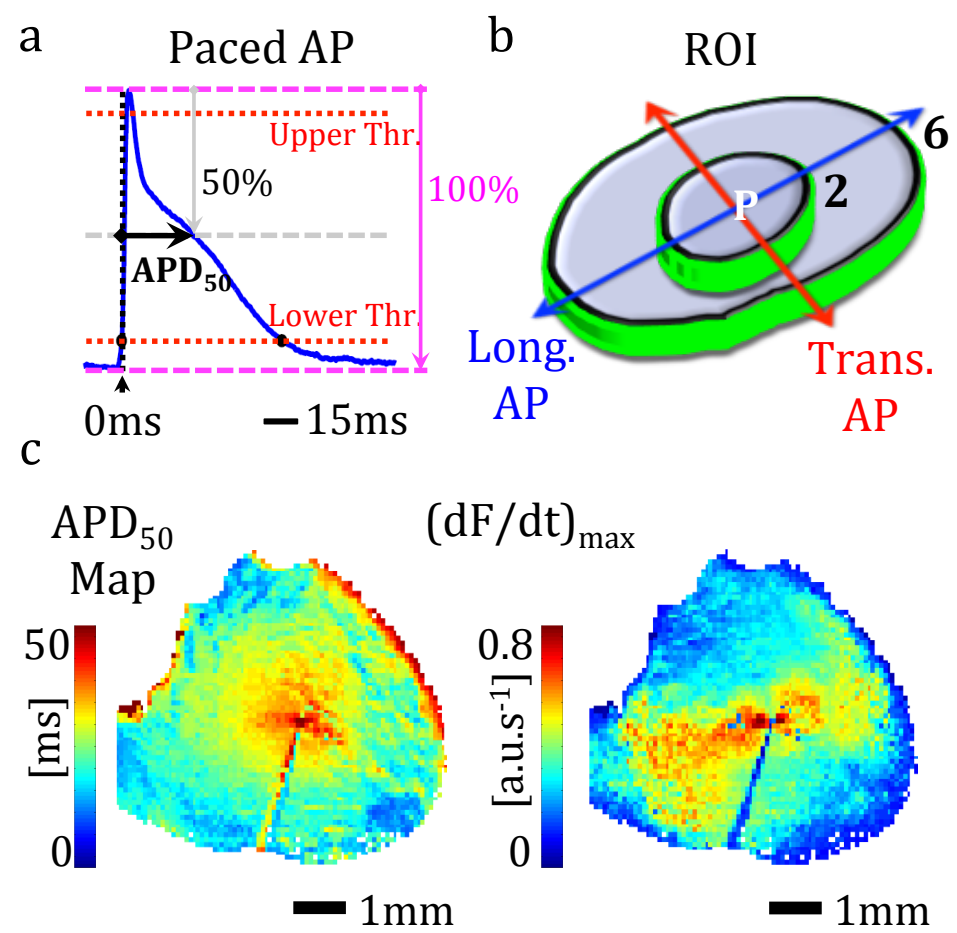

Whenever the analysis of $\mathrm{CV}$ or upstroke velocity $(\mathrm{dF} / \mathrm{dt})_{\max }$ is founded on a previously determined ROI (Figure 8b), which usually includes the isochrones number 2 to number 6 (i.e. the area covered by the traveling wave in its fastest speed configuration over a time interval of $4 \mathrm{~ms}$ ); hence avoiding pacing artifacts and boundary conditions (due to the ellipsoid shape of the heart being projected on a 2D surface). We use three different methods to quantify the CV components, parallel and perpendicular to the fiber direction (section 2.2.4).

$\mathrm{CV},(\mathrm{dF} / \mathrm{dt})_{\max }$ and APD dispersion maps are typically used in analyzing optical mapping data to characterize the local conduction velocity, upstroke velocity and APD at each pixel, respectively.

\subsubsection{Analytical Strategies In CV Analysis}

Least Squares Ellipsis Fitting (LSEF) Makes Use of Geometry. Our method exploits 2 criteria of myocardial propagation: 1) the elliptical properties of activation isochrones due to anisotropy; 2) the linear relationship between spatial isochronal points and activation times along two directions of propagation ${ }^{528}$. We extract contour lines from the activation map, for every $t_{k}, 1 \leq k \leq K t_{k}$, $1 \leq k \leq K$ as defined in the equation (5). To eliminate spurious isochrones due to misdetected 
activation times, only the longest contour line at each frame time $t_{k}$ is kept for further analysis. To each of these contours, ellipses are fitted which yield a set of major and minor axis lengths $a_{k}$ and $b_{k}$ respectively. Only one intermediate linear scaling regime can be found where $a_{k}\left(t_{k}\right)$ can be fitted to a line $a_{k} \approx v_{\|} \cdot\left(t_{k}+c_{\|}\right)$. Similarly, $v_{\perp}$ is obtained by fitting $b_{k} \approx v_{\perp} \cdot\left(t_{k}+c_{\perp}\right)$ for the same set of $k$ indices. The least square techniques recognize a set of parameters that minimize the distance between the isochronal data points and the ellipse ${ }^{531}$. The specificity of the fitted ellipse-solution is monitored by a cost function, which consists of arbitrary unit values plotted against time that characterize the closeness of the isochrones to the expected ellipse-solution, therefore relate a measure of the goodness of fit of the particular ellipse to the given set of data points. In the ideal conditions, the cost function is anticipated to be constantly zero for all times. Under experimental conditions, isochrones that return a cost function lower than unity are exclusively used for further analysis.

Plane Fitting (PF) Evaluates Velocities Locally. This method is based on averaging local wave fronts velocities, which are extracted using the well-known relationship ${ }^{532}$ :

$$
\vec{v}=\frac{\nabla f}{|\nabla f|^{2}}
$$

We use a method by Morley et al. to obtain $\nabla f$ in a robust way ${ }^{284}$. Instead of approximating the operator $\nabla$ by finite differences ${ }^{53}$, we fit a plane to the $n_{p} \times n_{p}$ square of pixels around every pixel $(i, j)$ and take the vector with the steepest slope of this plane as $\vec{v}(i, j)$. This is then used to calculate equation (6). Pixels with a divergence of the velocity field greater than $2 \mathrm{~cm} . \mathrm{s}^{-1}$.pixel ${ }^{-1}$ were excluded and they correspond to the location of wave initiation, which yields arbitrarily high wave speeds by default. By choosing the earliest activated pixels as a geometric centre $\left(x_{c}, y_{c}\right)$ for the created vector field, the pixels are sorted into bins according to the angle of the point $(i, j)$ with respect to the centre $\varphi(x, y)=\arctan \left(\left(y-y_{c}\right) /\left(x-x_{c}\right)\right)$. Using $N_{\varphi}=12$ bins, each bin has a width of $\Delta \varphi=\pi / N_{\varphi}$ and the angle of the $l^{\text {th }}$ bin is $\varphi_{l}=-\pi / 2+(l-0.5) \cdot \Delta \varphi$. After this procedure, each angle bin is associated with a mean wave speed

$$
v_{\varphi_{l}}=\left\langle\left\{|\vec{v}(i, j)| \mid \varphi_{l}-\Delta \varphi / 2<\varphi(i, j) \leq \varphi_{l}+\Delta \varphi / 2\right\}\right\rangle, 1 \leq l \leq N_{\varphi}
$$

Note that this definition only makes sense if the propagation is approximately symmetric: by using the arctan function, opposite sides with respect to the point of wave initiation are implicitly averaged into one angle bin. The fiber direction is then the angle $\varphi_{\tilde{l}}$, where $\tilde{l}=\arg \max _{1 \leq l \leq N_{\varphi}}\left(v_{\varphi_{l}}\right)$ is the index of the bin with the largest mean wave speed. The wave speed along the fiber direction is therefore $v_{\|}=v_{\varphi_{I}}$. A corresponding definition for the minimum mean wave speed yields a direction, which is approximately perpendicular to the fiber direction and therefore represents $v_{\perp}$.

Area Fitting (AF) Estimates Global Course of Activation. This method estimates velocities from the rate of increase of the area activated in each angle bin. If we define the $\varphi_{l}$ as above, using the activation map as defined in equation (5) we compute the area activated in each bin

$$
A_{\varphi_{l}}(t)=\sum_{\substack{(i, j) \\ a(i, j) \leq t \\(i, j) \in b i i_{l}}} \Delta x \cdot \Delta y, 1 \leq l \leq N_{\varphi},
$$

where $\operatorname{bin}_{l}$ contains all the points with $\varphi_{l}-\Delta \varphi / 2<\varphi(i, j) \leq \varphi_{l}+\Delta \varphi / 2$. The resulting function $A_{\varphi_{l}}(t)$ is fitted, for each bin $l$, to a quadratic function 


$$
f_{l}(t)=\frac{\pi}{N_{\varphi}}\left[v_{\varphi_{l}}\left(t-t_{\varphi_{l}}^{*}\right)\right]^{2}, 1 \leq l \leq N_{\varphi},
$$

where $v_{\varphi_{l}}$ and $t_{\varphi_{l}}^{*}$ are the result of a least-square fitting procedure and indicate the velocity and the apparent starting time (which might not be the time of pacing due to pacing artifacts) associated with the angle $\varphi_{l}$, respectively. The fitting is restricted to points from about $1.0 \mathrm{~ms}$ to $3.5 \mathrm{~ms}$ after the stimulation. As for PF, the longitudinal and transversal velocities are then estimated as the maximum and minimum of $v_{\varphi_{l}}$ with respect to $l$, respectively.

\subsubsection{Synthetic Data Used to Simulate Propagation}

Computer Simulation. The properties of the different methods were verified on synthetic data sets. In real experiments, the ideal anisotropic activation can be contaminated at the level of the acquired signal by measurement noise and at the level of the cardiac substrate by different levels of heterogeneity. To assess the performance of each of the methods, we carried out a series of numerical simulations to characterize the effects of those two particular parameters on robustness, accuracy and precision.

Data Model. Simulations were done on a two-dimensional, rectangular domain of $1 \mathrm{~cm} \times 1 \mathrm{~cm}$ in size using the Fenton-Karma model with Beeler-Reuter parameters ${ }^{534}$, that were modified $\left(\mathrm{g}_{f i}=12\right.$ and and $\mathrm{u}_{\mathrm{c}}{ }^{\mathrm{si}}=0.91$ ) to yield fast propagation velocities and a shorter action potential durations comparable to those in the experiments. The numerical resolution was $100 \mu \mathrm{m}$ in space and $10 \mu \mathrm{s}$ in time. Centered finite differences and a simple Euler step were used in space and time, respectively. A circular region with a radius of $200 \mu \mathrm{m}$ in the center of the domain was periodically stimulated at $10 \mathrm{~Hz}$ with $1.5 \mathrm{~ms}$ monophasic pulses to initiate outward-travelling waves. The fiber direction was chosen to be along the diagonal of the domain, in order to allow for longer propagation without the influence of the no-flux condition enforced at the boundary. Diffusivities of $1 \mathrm{~cm}^{2} . \mathrm{s}^{-1}$ and $0.5 \mathrm{~cm}^{2} . \mathrm{s}^{-1}$ were chosen parallel and perpendicular to the fibers, respectively. The total length of the simulations was 5s. A snapshot of the simulation was taken every $0.5 \mathrm{~ms}$ (corresponding to a sampling frequency of $2 \mathrm{kHz}$ ) and voltage data were inverted to create artificial optical mapping data.

Ideal Elliptical Propagation. We simulated the effect of perfect anisotropy in the cardiac tissue by an activation wave front initiated at a point-stimulation, and traveling to the boundaries as absolutely elliptical isochrones. Both maximal and minimal CVs were evaluated by the three strategies simultaneously over well-defined rings systematically scanning the entire activation field. Those rings are ROIs containing 5 consecutive isochrones similar to the region used in evaluating velocities in the experimental data. Conducive to substantially exploit all the simulated isochrones $(\mathrm{N}=23)$, we adopted the notion of a sliding interval that corresponds to that particular ROI shifted at increments of 1 isochrone, starting from the first to the $23^{\text {rd }}$ contour. To avoid artifacts in our analysis due to boundary effects, the outermost ring was restricted between the $12^{\text {th }}$ and $16^{\text {th }}$ contour lines. The curves plotted in the ideal case will serve as a platform against which the curves obtained from the non-ideal conditions will be contrasted.

Measurement Noise. Noisy data were constructed from the ideal case using additive Gaussian white noise: independent random numbers were drawn from a Gaussian distribution with standard deviation $\sigma_{\text {noise }}$ and added to each pixel in each frame of the artificial optical mapping data. The signal-to-noise ratio (SNR) was defined as the ratio $\sigma_{\text {signal }}^{2} / \sigma_{\text {noise }}^{2}$. Note that this original SNR ratio is significantly enhanced by the action of the aforementioned box filter. 
Simulated Heterogeneities. The effect of a heterogeneous cardiac substrate was implemented using a gaussian random field $G_{\sigma_{G}}(x, y)$ with a correlation length of $300 \mu \mathrm{m}$ and amplitudes measured as the standard deviation $\sigma_{G}$ of the ensemble of all points. The diffusion tensor $D$ of the simulation was then modified according to

$$
D(x, y)=D \cdot \exp \left[G_{\sigma_{G}}(x, y)\right] / \zeta,
$$

where $\zeta$ was chosen such that $\langle D(x, y)\rangle_{(x, y)}=D$, i.e. a constant mean velocity was maintained. With different realizations of these heterogeneous diffusion tensors, the numerical simulations were carried out as indicated above.

Statistics. Data are presented as mean \pm SM. Differences between groups were tested for statistical significance using the unpaired 2-tailed Student's $t$-test as indicated. $p$-values less than 0.05 were considered significant. 
a

Elliptical Anisotropic Activation

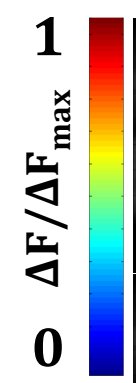

b

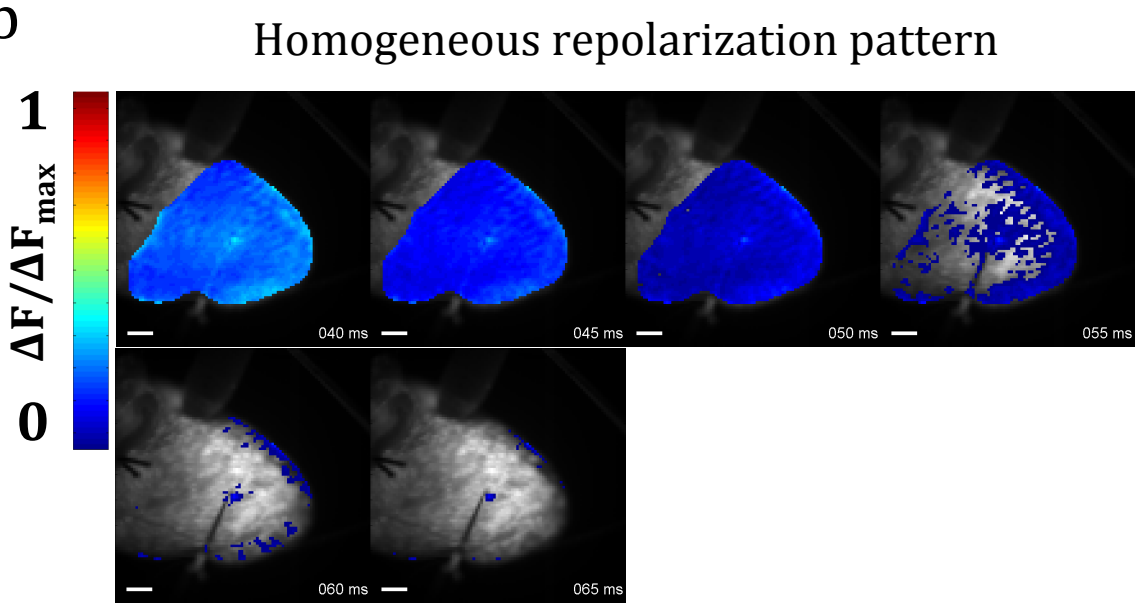

Figure 9. Depolarization (a) wave front followed by repolarization (b) projected on the epicardial surface (gray background) of the $L V$ free wall of a murine heart (WT). a. Mapping the amplitude of the activation wave front as it emanates from a point stimulation (pacing electrode placed at the center of the LV free wall). The wave propagates in an elliptical (anisotropic) fashion towards the LV boundaries within $8 \mathrm{~ms}$. At $15 \mathrm{~ms}$, all pixels are activated: cells have depolarized and entered the refractory phase (i.e. they are no longer excitable until repolarization is achieved). b. Repolarization is slower than activation and is homogenous throughout the heart. Such repolarization pattern indicates that the dispersion of repolarization in normal hearts is minimal, as nearly all cells regain excitability almost simultaneously. Annotations in figure: Scale bar $=1 \mathrm{~mm}$. Lookup-table normalized signal amplitude of the activation wave front. 
(This page is intentionally left blank) 



\section{Chapter 3}

\section{Results}

As indicated in the section 1.2.2, $\mathrm{Na}_{1} 1.5$ was demonstrated to associate with the dystrophinsyntrophin multiprotein complex (DSMC) in cardiac cells. The principal active molecular determinant of propagation in the ventricular myocardium is probably the availability of $\mathrm{Na}_{v} 1.5^{99}$, 368. However, the role of changes in $\mathrm{I}_{\mathrm{Na}}$ density on biophysical properties remains a controversial issue ${ }^{289}$, with varying reported effects among the different cardiac models investigated 535,536 . As described in the introduction ${ }^{\mathrm{lx}}$, the ventricular wall is far from being homogeneous with uniform electrical properties. In concordance with the discontinuous nature of electrical propagation in the heart, heterogeneity seems to be an intrinsic property of the ventricle that is not only observed at the different tissue levels comprising the wall ${ }^{262}$, but also prevails subcellularly with distinct profiles of activation in a network of connected cells ${ }^{537}$. Interventions, whether genetic (e.g. gain or loss of $\mathrm{Na}_{v} 1.5$ ) or pharmacological (e.g. Nav1.5 blockers), that alter propagation of an excitation wave in multicellular cardiac network may additionally extend the range over which many functional parameters, such as $(\mathrm{dF} / \mathrm{dt})_{\max }$ and APD, are inhomogeneous. Such increased dispersion in recovery from inactivation is believed to facilitate re-entrant excitation and renders the ventricular tissue prone to arrhythmias 263,538 .

The results presented below target three central questions: First, what impact does losing a pool of $\mathrm{Na}_{v} 1.5$ have on conduction in the medium and how does this possibly add to our understanding of cardiac dysfunction in DMD patients? Second, giving the importance of evaluating propagation instabilities in the cardiac tissue and its implication in facilitating arrhythmic patterns, how accurate are the existing analytical and numerical methods in detecting velocity changes of transient dynamical processes occurring on a millisecond scale? Would controversy regarding some models of cardiac electrical propagation stem from the actual limitations of methods used in optical mapping, particularly with their dependence on geometry, signal-to-noise ratio, initial and boundary conditions, and sensitivity to heterogeneities in the milieu? Third, a hallmark of reduced conduction in arrhythmogenic models is the simultaneous presence of repolarization abnormalities that amplify the electrical heterogeneity in the ventricle. How could heterogeneity be spatially and temporally characterized in models of loss or gain of Nav1.5 function, and how modifiable is this property under conditions of Nav1.5 blocking? Could Flecainide's "paradoxical" proarrhythmic effects at the multicellular level be dependent on contrasting modulation of Nav1.5 in different cardiac models?

Ix Refer to Introduction section 1.3.2 
In this context, the experimental framework of this thesis focused on characterizing electrical instabilities using high spatial-temporal optical mapping techniques in models harboring $\mathrm{Na}_{v} 1.5$ loss (the $m d x$ mouse model) or Nav1.5 gain of function ( $\triangle \mathrm{KPQ}$ model of LQTS3), each group with its corresponding WT counterparts, in ex-vivo conditions where isolated hearts were perfused with Flecainidelxi.

\subsection{Conduction Slowing in Hearts of $m d x$-Mice}

In a region of the heart, in which excitability and conduction is mainly supported by $\mathrm{I}_{\mathrm{Na}}$, an inaugural electrophysiological sequel of losing $\mathrm{Na}_{v} 1.5$ from the LM would probably be a global decrease in CV across the ventricle. Changes in anisotropic ratioxii (AR) due to clustering of the remaining functional $\mathrm{Na}_{\mathrm{v}} 1.5$ in the ID are less certain, possibly secondary to the dependence of anisotropy on other passive components, such as gap junctions (GJ) distribution ${ }^{199}, 539$, which hasn't been reported so far to be altered in the $m d x$ model. ECG recordings of $m d x$-mice showed a prolongation of the QRS interval ${ }^{237}$, which further suggests a general decrease in wave front propagation. In order to investigate whether $\mathrm{CV}$ is reduced in those hearts, optical mapping experiments of intact murine

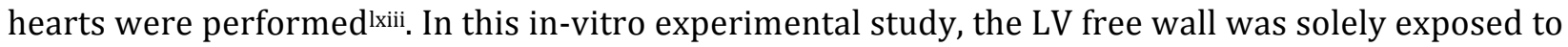
the optical path to provide a quasi-homogeneous field of tissue, with the pacing electrode positioned directly in the center to ensure the best possible elliptical configuration (Figure 10a). A comparison of the activation patterns obtained from WT and $m d x$-hearts are shown in (Figure 10b). Isochronal maps show that the maximal CV occurred in nearly the same direction in WT and $m d x$-hearts, but the activation patterns between the two groups are clearly non-identical. In order to delineate an unambiguous region where $\mathrm{CV}$ can be safely estimated, an iterative test-and-exclude process was performed to identify isochrones with points on the distance vs. time graph that do not deviate from the linear regime (Figure 10b, left). Regressions of the selected points were performed and the slopes represented the velocity of the wave front in that particular direction. In contrast to the WT hearts (Figure 10c), dystrophin-deficient hearts showed an average decrease of $31.4 \%$ in the transversal direction from $0.51 \pm 0.04 \mathrm{~m} . \mathrm{s}^{-1}$ to $0.35 \pm 0.06 \mathrm{~m} . \mathrm{s}^{-1}$ (WT, $\mathrm{n}=5 ; m d x, \mathrm{n}=8, p<0.001$ ) and along the longitudinal direction a decrease of $17.5 \%$ from $0.74 \pm 0.08 \mathrm{~m}_{\mathrm{s}} \mathrm{s}^{-1}$ to $0.61 \pm 0.11 \mathrm{~m} . \mathrm{s}^{-1}$ (WT, $\mathrm{n}=5$; $m d x, \mathrm{n}=8, p<0.05)$. AR increased on average by almost $10 \%$ in the $m d x$-group, however without a statistical significance (Figure 10c). Thus, under conditions mimicking the physiological resting mouse heart rate $(\sim 10-12 \mathrm{~Hz})$, the $m d x$ ventricle showed significant slowing of conduction in all directions, in concordance with the previously reported QRS widening237. The fact that the distribution and expression of $\mathrm{Cx} 43$ remains unaltered in the $m d x$ hearts 237,238 may explain the insignificant change in AR in those hearts. Using the ellipsis-fitting method we published these results as a collaborative work in Circulation Research $(2011)^{238}$. However, as different methods to evaluate CV using optical mapping were already published ${ }^{284}$, validation of our results using other methods was deemed necessary.

\footnotetext{
Ixi Refer to Introduction section 1.4 .7 for a complete description of the drug.

Ixii Anisotropic ratio is the ratio of the longitudinal and transversal velocities.

lxiii Refer to Experimental Methods section 2.1.2 for a complete description of procedure.
} 
a

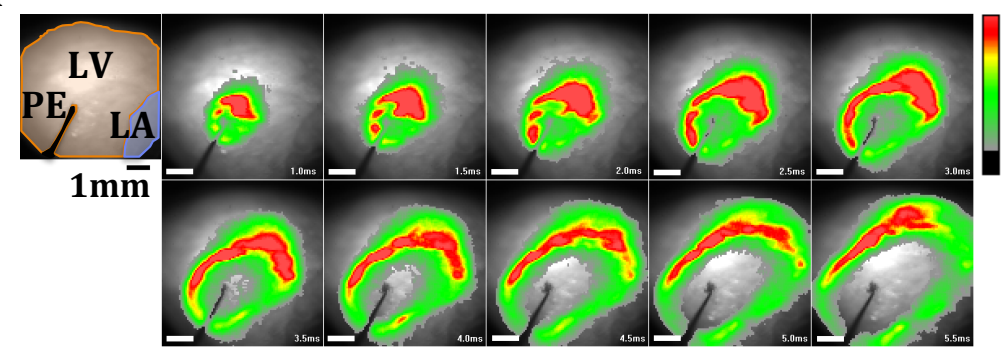

b
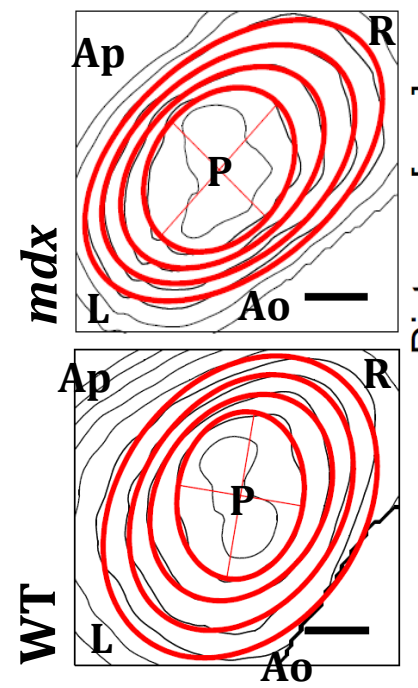

C

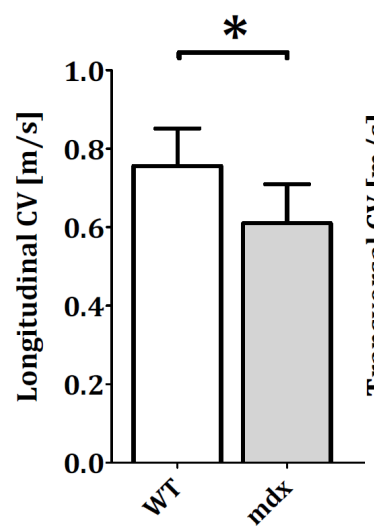

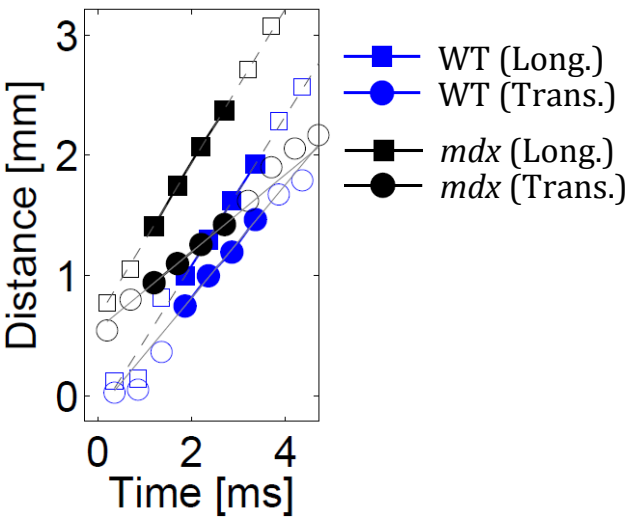

* $\quad p<0.05$

$* * * \quad p<0.001$
$1.5 \%$

$0.0 \%$

Figure 10. LV conduction slowing in $m d x$-hearts. a. Paced activation recorded from the $L V$ free wall of an $\boldsymbol{m d x}$-mouse heart at $0.5 \mathrm{~ms}$ time interval shows the expected elliptic spread, characteristic of anisotropy. The first derivative in time of the optical signal is calculated at each pixel, and as the wave front propagates the maximum of the derivative is colored according to the scale bar on the left. Gray color means refractory or quiescent tissue depending on its location relative to the wave front. b. Activation maps and estimation of $\mathrm{CV}$ by a linear regression. Isochronal contour lines are extracted from the activation recordings and ellipses are fitted successively to the interpolated pixels (left, red) with their corresponding data points (square $=$ longitudinal, circle $=$ transversal) for WT (black) and $m d x$ (blue). Filled symbols represent the selected data points used for the linear regression. The shallower slopes for the $m d x$ are indicative of slower conductions. Scale bar $=1 \mathrm{~mm}$. Ap $=$ Apex, $\mathbf{A o}=$ Aorta, $\mathbf{P}=$ Point stimulation, $\mathbf{R}=$ Right, $\mathbf{L}=$ Left, c. Bar graphs summarizing $\mathrm{CV}$ and $\mathrm{AR}$ averages. Statistical significance as indicated in figure, n.s., not significant.

Combining CV measurements to time parameters such as the RP or APD of a moving wave front, provides a distance parameter that is directly associated with the stability of a reentrant wave pattern ${ }^{100}$. The precise quantification of $\mathrm{CV}$ for the same cardiac substrate is often controversial 284 , 506, 507. Several methods have been implemented in the study of CV in normal or genetically altered mouse hearts under simple 238,508 and more complex activation patterns 509,510 . While they are appearing more frequently in publications, their implicit assumptions, errors and limitations are rarely subject of overt debate. Instead, they should be explicitly challenged and openly discussed, for if they are underestimated, results may be contradictory or misleading.

For this purpose, three analytical strategies were adopted as described in the Methods' section with their mathematical formulations. All these strategies yielded plausible results 238,284 . Despite their numerous advantages, they could suffer under non-ideal conditions from major drawbacks that 
could seriously complicate the quantitative evaluation of CV. The least-square ellipsis fitting (LESF), for instance, has a strong dependency on geometry. An overall deviation from the elliptical pattern will easily lead to large errors, whereas the vector field analysis (i.e. the plane fitting method, or PF) is prone to pick-up local heterogeneities in the activation medium and overtly estimate the velocity values, in addition to a significant loss in precision. The area fitting (AF) avoids dependence on geometry and sensitivity secondary to numerical derivatives, but hasn't been implemented as a potential strategy to measure $\mathrm{CV}$, possibly due to the fact that it mathematically returns a global parameter of spread, rather than an "actual velocity", in consequence it does not invest the full spatial resolution offered by optical mapping. A comprehensive comparison of the proposed methods concludes this section. The validity, advantages, sensitivity and limitations of these methods are discussed in the next chapter. However, this study reviews evidence that analytical methods may not always be applicable, and when they are not, the obtained results may be misleading.

\subsubsection{LSEF: an Anisotropy-Dependent Method}

In order to avoid investigator bias, an additional component was added to the LSEF method. The need to objectively determine the area where CV can be safely evaluated, isochrones that particularly minimize the cost function were included, and their corresponding data points were exclusively used for the linear regression to evaluate the CV within this ROIxiv. The observed condensation of isochrones in the $m d x$-model is appropriately exploited by LSEF. In Figure 11b, we notice that compared to the WT activation in Figure 11a, tightening of the spatial map for the $m d x$ heart is translated into smaller slopes for both major and minor directions of propagation. Because first isochrones are prone to pacing artifacts, noted by the irregularities in the shape, that possibly lead to arbitrarily high velocities, they were excluded from the linear regression analysis (Figure $11 \mathrm{~b}$, right). CV using LSEF was reduced on average by $24.7 \%$ along the longitudinal (WT, $\mathrm{n}=17 ; m d x$, $\mathrm{n}=17 ; p$-value $<0.0001$ ) and $21.1 \%$ along the transversal ( $p$-value $<0.0001$ ) directions in the $m d x$ hearts relative to the WT control group (Figure 11c), with no significant change in AR (Figure 11d). Consequently, this part of the study shows that the number of experiments used for the previously published results wasn't a limiting factor. The percentage decrease in velocities was within the previously reported ranges using this method. Nevertheless, the higher number of experiments has mainly strengthened the statistical significance of our results.

\subsubsection{PF: Local Calculation of Velocity Wave Fronts}

The colored vector fields in Figure 12a and b show the anisotropic activation patterns for WT and $m d x$-hearts respectively, both representing a well-behaved configuration almost similar to the ideal simulation model. Nevertheless, Figure $12 \mathrm{~b}$ reveals a slower propagation in the $m d x$-hearts reflected by a longer time of activation when compared to the WT. Each vector combines a direction and amplitude at each pixel. The vectors emanate from the pacing site in an anisotropic manner, regardless of the direction of the underlying epicardial fibers. Only within the ROI delineated above (i.e. between $t_{1}=0.5 \mathrm{~ms}$ and $t_{6}=2.5 \mathrm{~ms}$ ), the assumption of homogeneous symmetrical fields around the pacing site stands. Therefore the resulting vectors are binned based on a geometric center that coincides with the tip of the pacing electrode, leading to a continuous set of mean velocities spanning the directions from $90^{\circ}$ to $270^{\circ}$ (Figure $12 \mathrm{a}$ and b) from which the fastest and slowest CV in the medium are picked up. This method reported a significant CV decrease of $24 \%$ along the maximal ( $p$-value $<0.0001)$ and $19.5 \%$ along the minimal $(p$-value $<0.0001)$ directions in the $m d x$ hearts relative to the WT group (Figure 12c), with no change in AR (Figure 12d).

Ixiv Refer to Experimental Methods section2.2.3 and Figure 8 for the current definition of ROI in this context. 


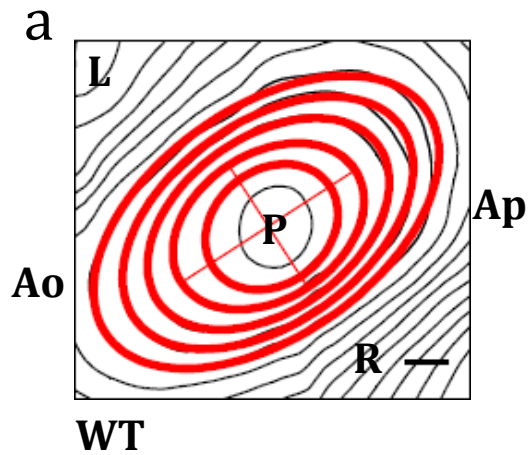

b

Ao

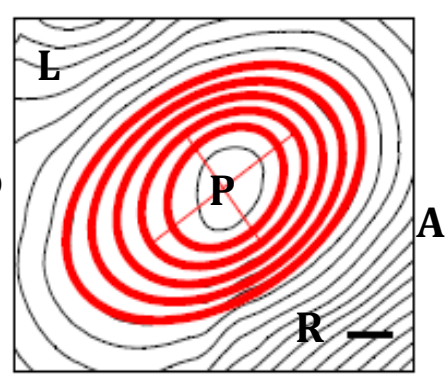

$\operatorname{mdx}$
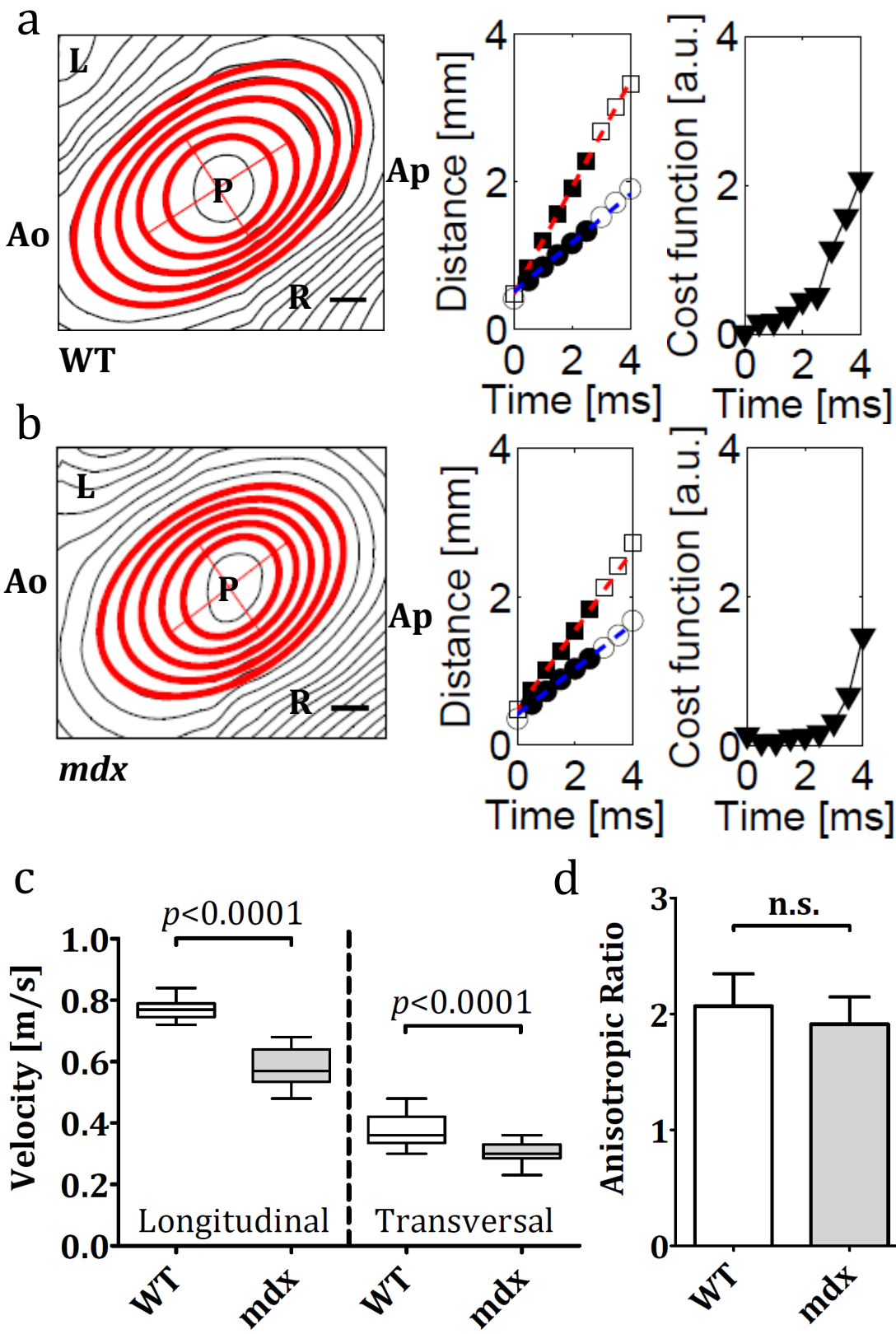

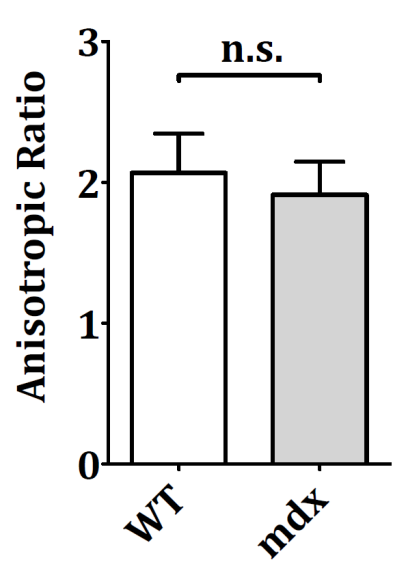

Figure 11. Least-square ellipsis fitting method (LSEF). a. From the WT activation map we extract isochronal contour lines; ellipses are successively fitted (on the left, in red) to the interpolated pixels, which return a set of major and minor axes lengths. The center of the ellipses coincides with the actual point of stimulation (P). Longitudinal (square) and transversal (circle) isochronal points, deduced from the bilaterally averaged major and minor axes of the ellipses respectively, are plotted in a distance vs. time graph (middle). In an exclusively intermediate linear scaling regime (filled symbols), isochronal points are fitted along the direction of propagation, yielding the respective velocity value. Points that deviate from the linear behavior (open symbols) were excluded from the regression. $\mathrm{V}_{\max }=0.72 \mathrm{~m} . \mathrm{s}^{-1} ; \mathrm{V}_{\min }=$ $0.35 \mathrm{~m} . \mathrm{s}^{-1}$. The goodness of fit for each of the ellipses is estimated using a cost function (right). Ellipses that return a cost function value less than 1 are exclusively considered for further analysis. Scale bar $=0.5 \mathrm{~mm}$. Ap=Apex; Ao=Aorta; $\mathbf{R}=$ Right, $\mathrm{L}=$ Left. b. Same analysis used for $\boldsymbol{m d x}$-activation map. Estimated $\mathrm{V}_{\max }$ $=0.54 \mathrm{~m} \cdot \mathrm{s}^{-1} ; \mathrm{V}_{\min }=0.30 \mathrm{~m} \cdot \mathrm{s}^{-1}$. c. Box plots with whiskers showing significantly reduced longitudinal and transversal CV. d. Bar graphs showing no difference in AR, n.s. not significant as indicated.

\subsubsection{AF: An Estimation of Global Conduction Method}

As the cardiac excitation propagates from the tip of the pacing electrode, it separates the depolarized and quiescent myocardium. The temporal evolution of the activated area can be visualized in Figure 13a and b (left) for both WT and $m d x$-hearts respectively, where the white region spreads over the resting black myocardium. The symmetry around the pacing site is still conserved at $\mathrm{t}_{6}=2.5 \mathrm{~ms}$, which allows for a symmetrical segmentation of the map along 16 different directions of propagation, evaluated in pairs resulting in 8 time series (panels a and $b$, middle), to each of which a quadratic function is fitted. This function returns 8 effective velocity measures from which only the fastest and slowest velocities are picked up (panels a and b, right). We report a 
global velocity decrease of $24 \%$ along the fastest ( $p$-value $<0.0001)$ and $17.5 \%$ along the slowest directions ( $p$-value $<0.01$ ) between the 2 experimental groups.

Figure 12. Plane fitting method (PF). a. and b. Vector fields calculated from the previously shown activation maps for WT and $m d x$ hearts respectively. Each black vector is characterized with both a direction, which corresponds to the direction of propagation of the local wave front and a scalar amplitude, which corresponds to the $\mathrm{CV}$ at that particular pixel. The pacing site, taken to be the geometric center of the vectorial map, coincides with the center of the polar plot (right). Vectors are projected into 24 different orientations going from $0^{\circ}$ to $360^{\circ}$ at a bin width of $15^{\circ}$. Average velocities (blue line) along with the standard deviations (red dotted lines) are calculated in each direction by averaging each bin and its diametrically opposite counterpart, yielding velocity vectors oriented from $90^{\circ}$ to $270^{\circ}$, from which the maximum and minimum scalar amplitudes (the large and small black arrows in the polar plots) are automatically picked up and represent the fastest and slowest CVs in the medium respectively. WT: $\mathrm{V}_{\max }$ $=0.7 \pm 0.05 \mathrm{~m} . \mathrm{s}^{-1} ; \mathrm{V}_{\min }=0.35 \pm 0.04$ $\mathrm{m} . \mathrm{s}^{-1} \cdot m d x: \mathrm{V}_{\max }=0.55 \pm 0.08 \mathrm{~m} . \mathrm{s}^{-1}$; $\mathrm{V}_{\min }=0.31 \pm 0.03 \mathrm{~m} . \mathrm{s}^{-1}$. For clarity, only 1 out of 2 calculated vectors are drawn here. Horizontal scaling arrow (upper, left), magnitude $=1$ $\mathrm{m} . \mathrm{s}^{-1}$. Scale bar $=1 \mathrm{~mm}$. Color scale bar $=$ time scale of activation in $\mathrm{ms}$ $\left[\begin{array}{ll}0 & 10\end{array}\right]$ Ap=Apex; Ao =Aorta; R=Right, L=Left. c. Box plots with whiskers showing significantly reduced $\mathrm{CV}$ along the maximal and the minimal directions between the 2 experimental groups. d. Bar graphs showing no difference in $A R$, n.s. not significant as indicated.
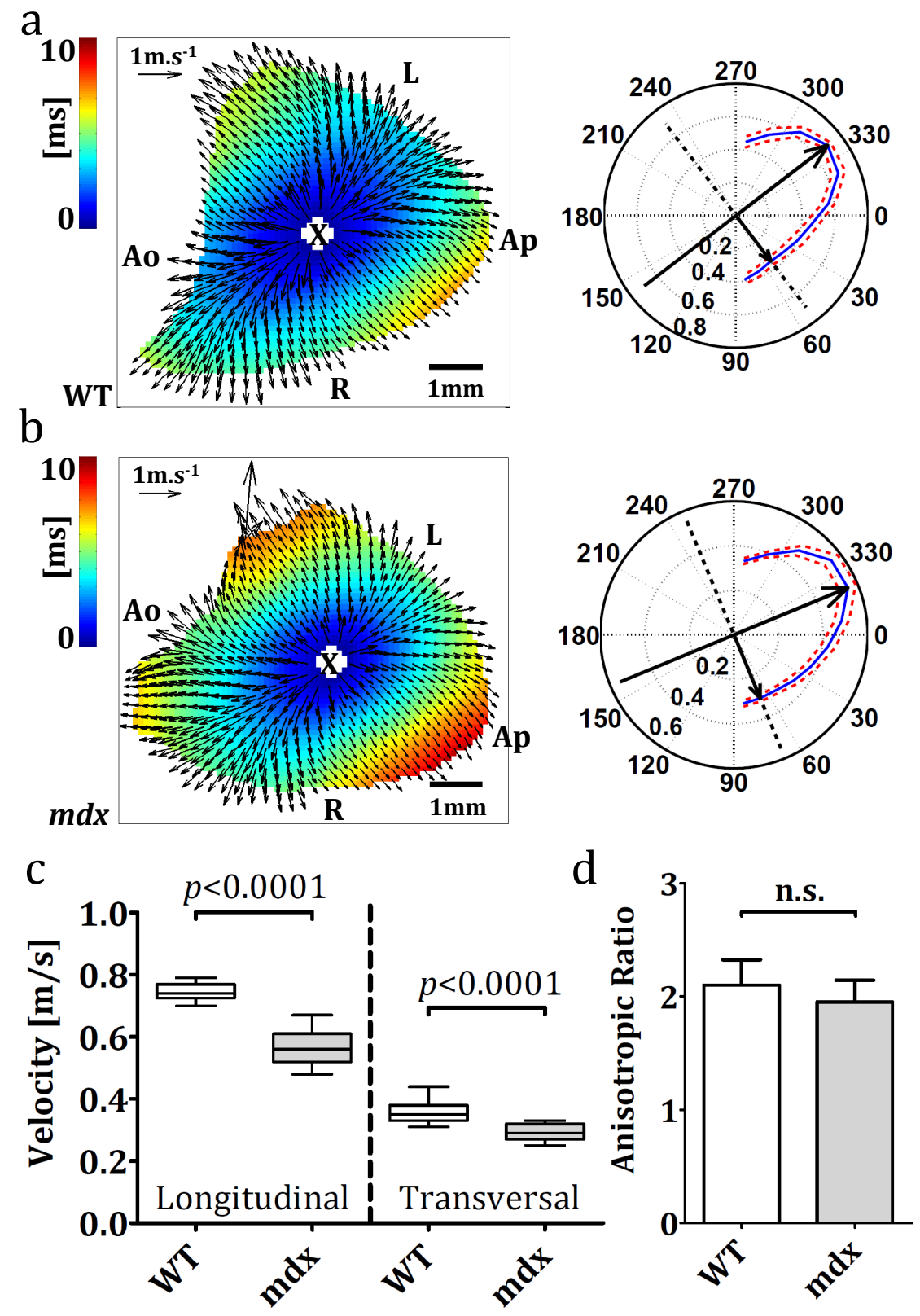

These results consistently show a CV decrease in the mutated group. Nonetheless, in all previous measurements, activations were carefully selected not to deviate from anisotropy, which in return might be introducing a bias component in restricting the analysis within the safety margins of a well-defined geometric propagation. Hence, this agreement between the methods needs to be further questioned. It certainly adds to the validity of each of the implemented methods; however 
does not reveal a direct comparison between them, nor it does show how well the methods would agree in more complicated activation patterns.

a

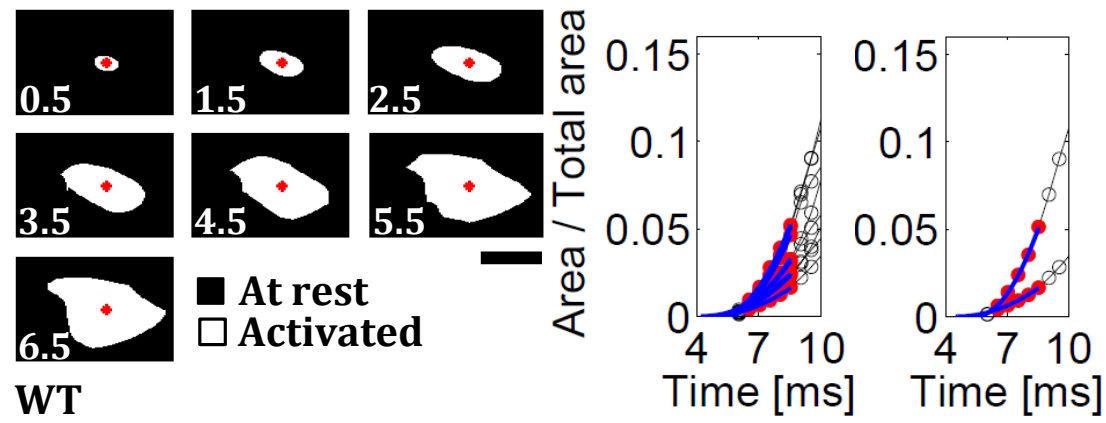

$\mathrm{b}$

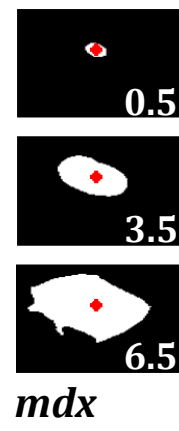

C
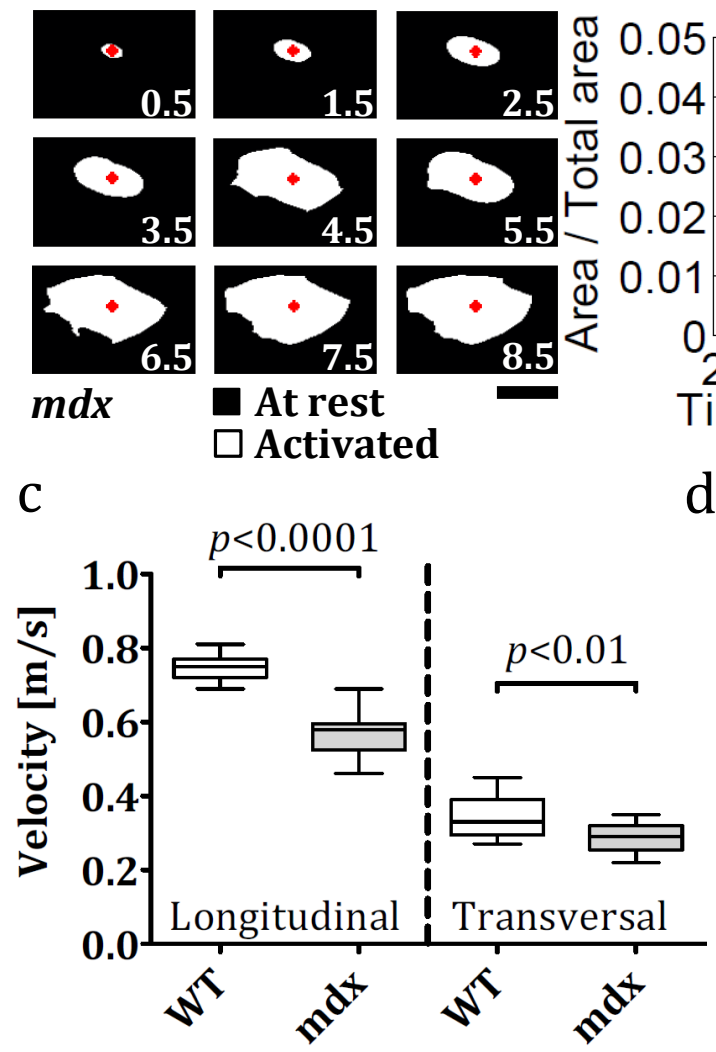

$\begin{array}{ll}\stackrel{\varpi}{\otimes} & 0.05 \\ \stackrel{0}{\sigma} & 0.04\end{array}$
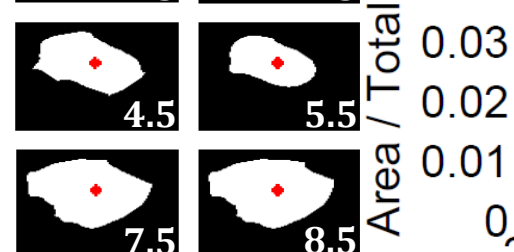

At rest Activated

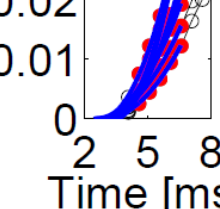

Time $[\mathrm{ms}]$

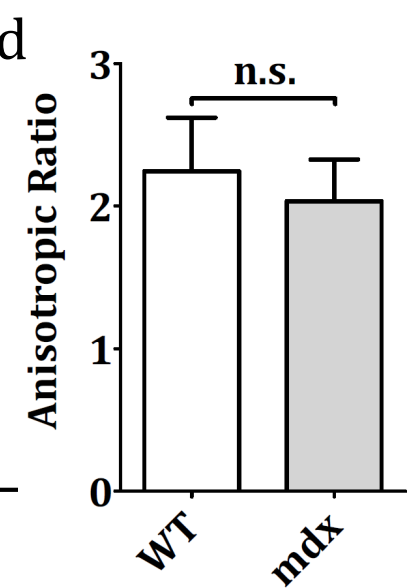

Figure 13. Area Fitting method (AF). a and b. The temporal evolution of the area activated by the propagating wave from the point of stimulation (red dot) at the center of the LV to the boundaries for WT and $m d x$ hearts respectively (left). For simplicity, snapshots of the activated area are shown at $1 \mathrm{~ms}$ intervals. Scale bar $=3 \mathrm{~mm}$. The isochronal map is segmented into 16 bins of different angles of propagation. By evaluating simultaneously each bin with its contralateral side, a total of 8 time series are derived (middle), created from the extracted isochronal points as the activated area expands in those particular orientations. A quadratic function (blue line) is fitted to selected points in each of the time series (red symbols), yielding an effective velocity measure representing a global course of activation in that particular direction; from which the fastest and slowest rates of activation are selected (right). WT: $\mathrm{V}_{\max }=0.70 \mathrm{~m} \cdot \mathrm{s}^{-1} ; \mathrm{V}_{\min }=0.32 \mathrm{~m} \cdot \mathrm{s}^{-1} \cdot \mathrm{mdx}$ : $\mathrm{V}_{\max }=0.53 \mathrm{~m} . \mathrm{s}^{-1} ; \mathrm{V}_{\min }=0.29 \mathrm{~m} \cdot \mathrm{s}^{-1}$. c. Box plots with whiskers showing significantly reduced velocities along the fastest and slowest directions. d. Bar graphs showing no change in AR, n.s. not significant.

\subsubsection{Deviation from Anisotropy: Failure of Methods' Convergence}

Three experiments were further performed with the $\triangle \mathrm{KPQ}$ mice and maps with an initial anisotropic spread were exclusively chosen. Similar outcomes were reproduced with these Nav1.5 mutated hearts, as the previous two experimental groups: As expected, the methods yielded comparable results under anisotropic conditions (Figure 14a). The reported $\mathrm{V}_{\max }$ using PF is almost $10 \%$ higher than the ones by LSEF and AF because the method was chosen to explore the full circumference of the ROI, without symmetry consideration around the geometric center (as opposed to the previous analysis done for the $m d x$ and WT experiments). The method is therefore 
expected to pick up the maximal velocity from the bin with the highest calculated mean. For a quick check, binning both sides returned $V_{\max }=0.84 \pm 0.02 \mathrm{~m} . \mathrm{s}^{-1}$ instead of $0.95 \pm 0.02 \mathrm{~m} . \mathrm{s}^{-1}$, which is almost identical to the values reported by LSEF and the AF (See caption). Application of Flecainide distorted anisotropy (Figure 14b) and pushed the methods to diverge considerably in their final results. The distortion seen in the activation map is not due to pacing artifacts, because deviations from anisotropy were reproducible in the other two $\triangle \mathrm{KPQ}$ hearts treated with this Nav1.5-blocking agent. Quantitatively, this deviation is characterized by the high values of the cost function and the irregular shape of the mean velocities line in the polar plot along with their respective high SD in each bin.

Figure 14. Inaccuracy in evaluating CV due to a heterogeneous activation after 5 min of treating $\triangle \mathrm{KPQ}$ heart with Flecainide. a. Under control conditions, $\triangle K P Q$ shows an elliptical activation; Scale bar $=1 \mathrm{~mm}$. LSEF: nicely fitted ellipses (up, middle) return near 0 values for cost function. Scale bar $=0.5 \mathrm{~mm}$. The linear fit (right) returns $\mathrm{V}_{\max }=$ $0.85 \pm 0.01 \mathrm{~m} . \mathrm{s}^{-1} ; \mathrm{V}_{\min }=0.38 \pm 0.01 \mathrm{~m} \cdot \mathrm{s}^{-1}$. (bottom, left). Vectors within ROI are emanating from the point stimulation. $1^{\text {st }}$ isochrone not included in ROI, hence empty center in vector field. Horizontal arrow (magnitude for scale) $=1 \mathrm{~m} \cdot \mathrm{s}^{-1}$. Scale bar $=1 \mathrm{~mm}$. Polar plot (bottom, middle) of velocity values (blue) calculated within $15^{\circ}$ bin (based on geometric angle, see text) running from 0 to $2 \pi$ and their respective SD (red dotted line). Note the almost elliptical shape of the blue line, i.e. an anisotropic distribution of velocities: $\mathrm{V}_{\max }=$ $0.95 \pm 0.02 \mathrm{~m} . \mathrm{s}^{-1} ; \mathrm{V}_{\min }=0.35 \pm 0.01 \mathrm{~m} . \mathrm{s}^{-1}$. AF (bottom, right) returns $V_{\max }=0.82 \mathrm{~m} . \mathrm{s}^{-1}$; $V_{\min }=0.34 \mathrm{~m} \cdot \mathrm{s}^{-1}$. b. Induced activation pattern heterogeneity in substrate after $\mathbf{5 m i n}$ drug perfusion. Distortion of AM (up, left) disables a good-fit of ellipses (middle) that increases the cost function, rendering LSEF inaccurate despite a good linear fit of data points in distance vs. time graph (up, right) with $\mathrm{V}_{\max }=$ $0.72 \pm 0.09 \mathrm{~m} . \mathrm{s}^{-1} ; \quad V_{\min }=0.32 \pm 0.05 \mathrm{~m} . \mathrm{s}^{-1}$. Scale bar $=0.5 \mathrm{~mm}$. Vectors (bottom, left) blow up in concordance with spaced isochrones in the longitudinal direction. Horizontal arrow (magnitude for scale) $=1 \mathrm{~m} \cdot \mathrm{s}^{-1}$. Scale bar $=1 \mathrm{~mm}$. Note the irregular star-shaped blue line in polar plot, with widening of SD curve: $\mathrm{V}_{\max }=$ $1.4 \pm 0.12 \mathrm{~m} . \mathrm{s}^{-1} ; \mathrm{V}_{\min }=0.34 \pm 0.08 \mathrm{~m} . \mathrm{s}^{-1}$. AF returns middle values between LSEF and $P F$ for $V_{\max }\left(0.9{\mathrm{~m} . \mathrm{s}^{-1}}^{-1}\right.$, however a smaller $V_{\min }\left(0.16 \mathrm{~m} \cdot \mathrm{s}^{-1}\right) . \mathbf{A p}=$ Apex, $\mathbf{A o}=$ Aorta, $\mathbf{P}=$ pacing, $\mathbf{R}=$ Right, $\mathbf{L}=$ Left.
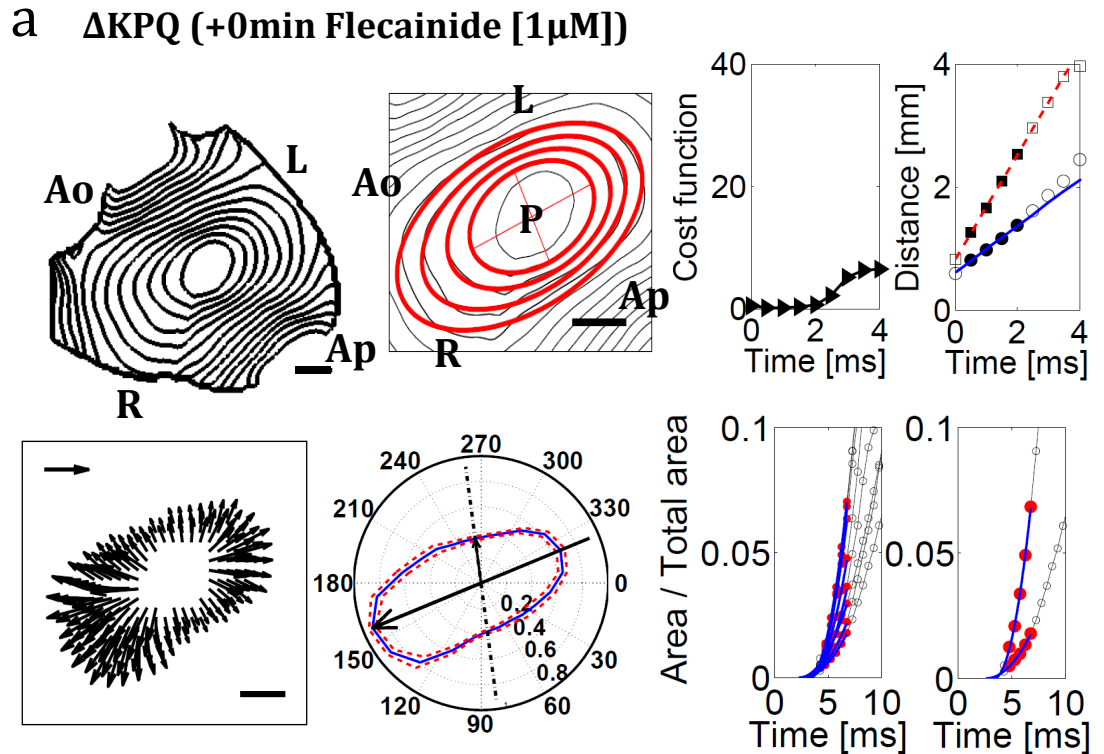

\section{$b$}

$\Delta \mathrm{KPQ}(+5 \mathrm{~min}$ Flecainide $[1 \mu \mathrm{M}])$
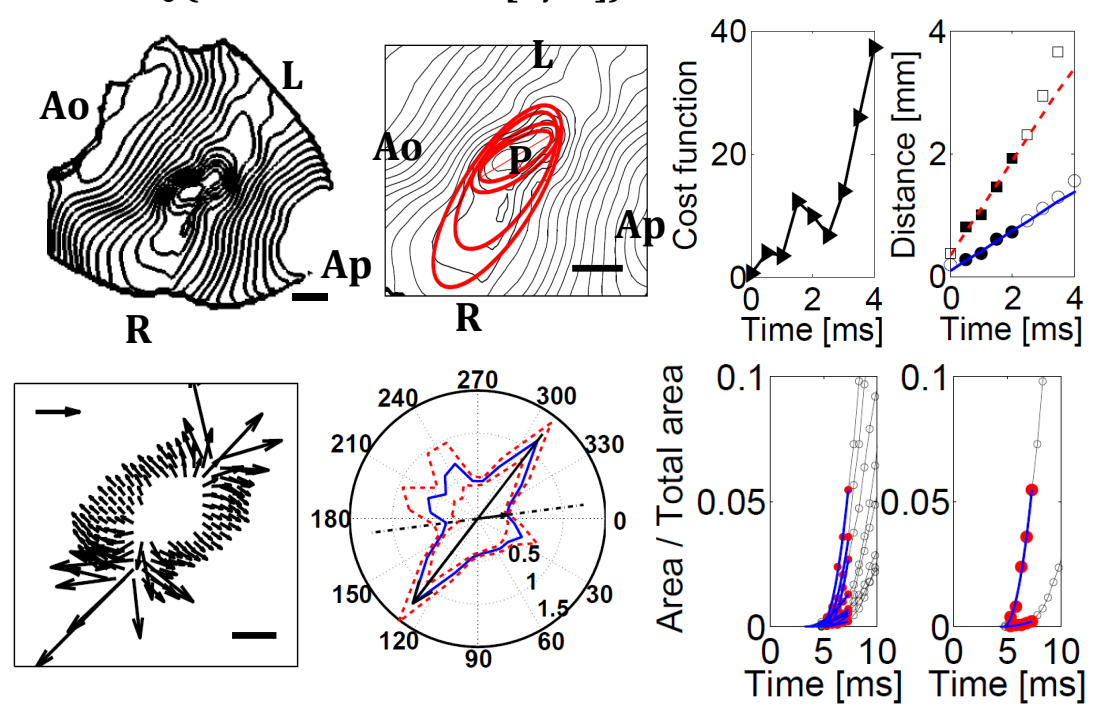


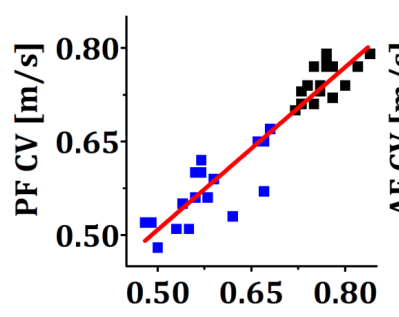

LSEF CV $[\mathrm{m} / \mathrm{s}]$

Slope $=0.87 \pm 0.05$

$\mathbf{R}^{2}=\mathbf{0 . 9 0}$

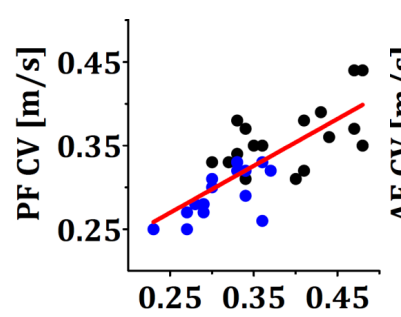

LSEF CV $[\mathrm{m} / \mathrm{s}]$

Slope $=0.57 \pm 0.08$

$\mathbf{R}^{2}=0.59$

\section{- WT max. CV \\ - mdx max. CV}

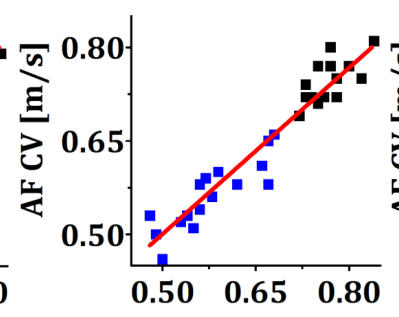

LSEF CV $[\mathrm{m} / \mathrm{s}]$

Slope $=0.89 \pm 0.05$

$\mathbf{R}^{2}=0.92$

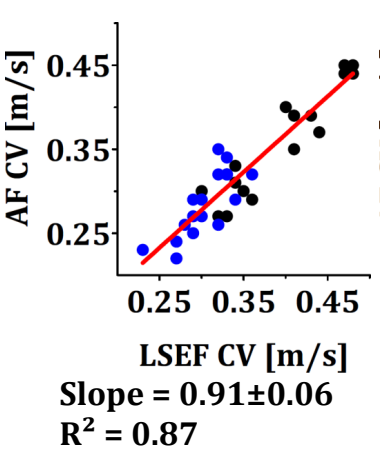

- WT min. CV

- $\operatorname{mdx}$ min. CV

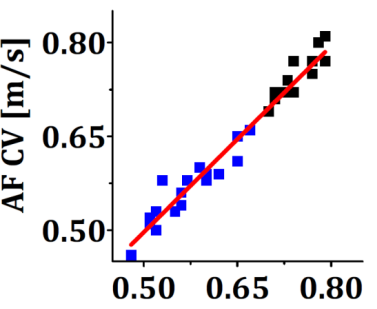

PF CV $[\mathrm{m} / \mathrm{s}]$

Slope $=0.99 \pm 0.03$

$\mathbf{R}^{2}=0.97$

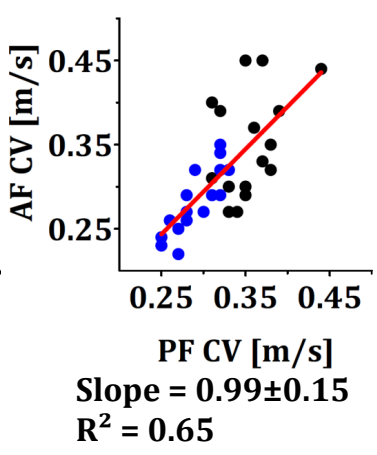

Linear

Regression
Figure 15. Correspondence among LSEF, PF and AF methods. Regressions lines (red) are drawn through velocity values for a direct comparison between the methods' outcomes $V_{\max }$ (up, squares) and $V_{\min }$ (bottom, circles) from both WT (black) and $m d x$ (blue) groups. For high values of the coefficient of determination $\left(\mathrm{R}^{2}>0.85\right)$ the majority of dots assumes a long narrow pattern, with a target slope $\sim 1$. Two cases where $R^{2}$ is relatively low appear in the graphs correlating $V_{\text {min }}$ values: First, between the PF and LSEF with a slope considerably far from 1 (bottom left) and second, between $\mathrm{AF}$ and $\mathrm{PF}$ (bottom, right) with a slope $\sim 1$. These correlations mainly show that the methods are independent and non-biased in estimating velocity values in the same preparation.

In order to determine the relationship between the different outcomes of the methods and how well the values, which also include the errors associated with the methods, are correlated, velocity values are plotted on a graph that combines two methods at a time, separating $V_{\max }$ and $V_{\min }$ being the two variables extracted with each method. In the most ideal conditions (i.e. ideal methods and ideal substrates), both the determination coefficient $\left(\mathrm{R}^{2}\right)$ and slopes of all combinations must be strictly equal to unity. If the methods were independently estimating the velocities (i.e. non biased) but still correlated well, both $\mathrm{R}^{2}$ and slopes should be close to one. Mixing the two groups (WT, $m d x$ ) and treating them as one population considerably increases the magnitude of the determination coefficient $\mathrm{R}^{2}$ in almost all combinations of methods, mainly for the $\mathrm{V}_{\max }$, than when each group is treated separately (Figure 46). This could be due to increasing the number of variables in the plot on one hand. On the other hand, the WT and $m d x$ represent two sets with completely independent measurements, and the velocities in each group show sizeable statistical differences for all methods. When the magnitude of the $\mathrm{R}^{2}$ is high, the slope is also substantially close to 1 , an expected finding for independently working methods. Some exceptions are relevant: the first is found in the plot that combines the $V_{\min }$ values of PF vs. LSEF (with $\mathrm{R}^{2}=0.59$ and slope $=0.57 \pm 0.08$; Figure 15 , bottom, left) and the second in the plot combining $\mathrm{V}_{\min }$ values of the AF vs. PF (with $\mathrm{R}^{2}=0.65$ and slope $=$ $0.99 \pm 0.15$; Figure 15, bottom, right). In the first plot, if the slope were the only determinant of the agreement between the methods, then PF will constantly return a lower value than LSEF. However, considerable point scattering indicates that large errors in the measurements are non-correlated. In the second plot, intermingled data points (low $\mathrm{R}^{2}$ ) suggest that either the methods randomly disagree over the same actual velocity value (hence, the slope is almost one), or the measurements and their errors are completely uncorrelated. A third possibility could be due to the fact that the natural variability of $V_{\min }$ is smaller than the one of $V_{\max }$; hence harder to measure by default. In summary, these methods show unbiased behavior is estimating velocities along with uncorrelated errors. Therefore, increased accuracy is achievable with the use of several methods simultaneously. 
The working range of the three methods was further challenged using synthetic data sets. The dependence of the LSEF and PF on the capacity to produce valid activation maps is not the case for $\mathrm{AF}$, which is completely independent of such an assumption. This means, keeping other parameters fixed, increasing noise levels would limit the reasonable use of LSEF and PF, without necessarily causing similar effects on the behavior of AF method. The fact that all methods perform plausibly well under homogeneously and fairly anisotropic conditions cannot be extended to include nonhomogeneous conditions without challenging the methods with increasing level of heterogeneity. LSEF being restricted to a linear regime and the AF to an overall area increase are intrinsic properties that would limit the sensitivity of these methods to heterogeneity. In contrast, PF has the intrinsic property of evaluating velocity vectors locally, hence can incorporate progressively higher SDs to the calculated velocities as a measure of increasing heterogeneity.

\subsubsection{Simulating the Ideal Condition}

In both Figure 16a and Figure 17a (outmost left column), activation contours are calculated from the simulation of a perfectly homogeneous and anisotropic medium. The fitted ellipses in red are perfectly superimposed on the simulated isochrones. The calculated vectors in all pixels are protruding outward from the point of stimulation in an almost parallel fashion in each direction (Figure 16a, outmost left column, bottom). In the polar plot (similar to the polar plot in Figure 14), average velocities, when spanning angles from 0 to $2 \pi$ within the ROI, form a perfect ellipticallyshaped line, with near 0 standard deviation values for the mean velocities in all bins (Figure 17b, right). This indicates an ideal distribution of velocities with the highest possibly acquired precision by the method. In light of these findings, all curves representing the calculated velocities by each of the three methods are almost congruent point-by-point (Figure 18a, left). It can be assumed that this highly organized pattern offers an ideal basis for a straightforward comparison of the CVs over each interval of interest in the activation medium, for all the methods combined; hence providing a confirmation for the observed agreement between the methods for the experimental data.

\subsubsection{Robustness to Noise}

Estimating CV is adjoined with the challenge of accurately distinguishing activation wave fronts. In Figure 16a, the noise level was systematically added at each pixel in order to assert the methods' ability to cope with high noise levels. Isochronal contours become more rugged as the SNR decreases, despite the applied smoothing and filtering processes, with appearance of "dark spots" or "buds" at SNR $=0.03$, due to misdetection of activation times in a random fashion leading to spurious isochronal lines between neighboring pixels. These small-scale irregularities seem to average out, keeping the overall geometry readily detectable by the ellipse fitting algorithm, observed in the stability of the fitted ellipses. The PF remains robust, with slowly increasing but still insignificant deviations in vectors' directions, keeping amplitude fluctuations in a comparable range with both the LSEF and AF (Figure 18a, right). Except for the earlier points (SNR $=0.03$ ), the velocity curves eventually converge. The first 4 points in the graph diverge, due to different velocities calculated over these intervals. Both the LSEF and AF underestimate the mean CV compared to the ideal case, with a 15\% decrease along the longitudinal direction and $9 \%$ along the transverse direction for the LSEF, and 5\% decrease along the maximal direction and 12\% along the minimal direction for the AF. The PF reports very similar results for the mean velocities at SNR=0.03, when compared to the ideal condition. However, the noise effect is reflected in the increasing SD, reaching $11 \%$ in the noisy set compared to $1-2 \%$ in the ideal case. When SNR reaches 0.01 , the wave spread is completely blurred with noise, leading to utterly random detections of activation times at each pixel (Figure 16a, rightmost column). Failure to delineate any geometry in the activated pixels causes a chaotic plotting of ellipses. The PF shows overtly larger and smaller amplitude vectors in a 


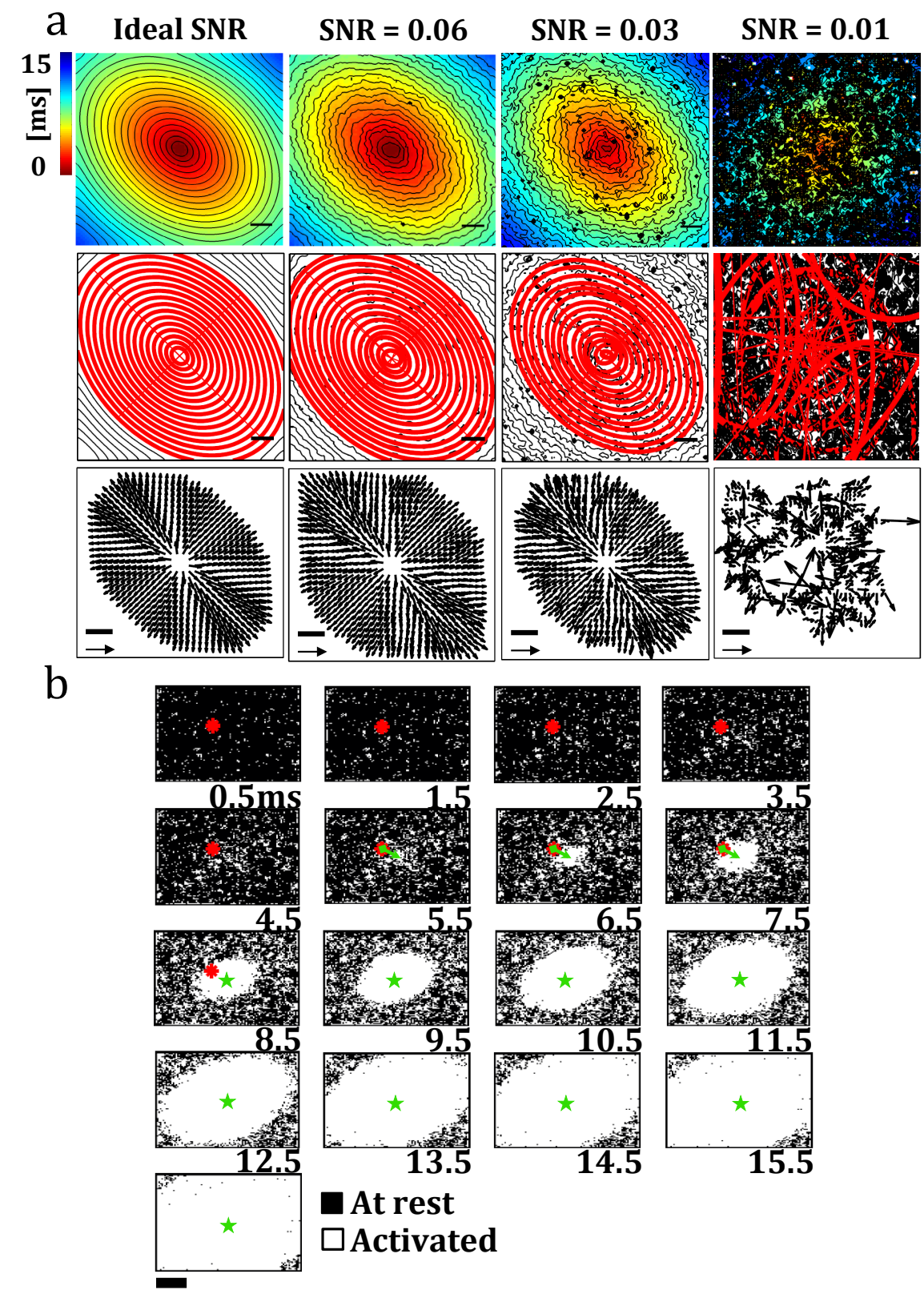

Figure 16. Comparing the methods using computational modeling for increasing noise. a. Fitting of ellipses and planes to activation maps with incremental noise. Notice the increasing roughness in the isochronal lines due to variable detection of activation times. Small-scale deformities are well tolerated by LSEF. As SNR degrades to 0.03 , the algorithm is incapable of detecting with enough precision the AP upstroke at each pixel, which favors a continuous loop of activation time misdetections, causing black dots that "bud" from the isochrones. As for $\mathrm{PF}$, apparent deviations in the resulting vectors at each pixel are balanced out by binning. The contours become entirely obscured with "budding" artifacts when SNR drops to 0.01 , the level at which both LSEF and PF fail to return reliable results. Horizontal scaling arrow $=$ vector magnitude $=1 \mathrm{~m} \cdot \mathrm{s}^{-1}$. Scale bar (in all maps) $=1 \mathrm{~mm}$. b. Global activation pattern seen at $\mathbf{S N R}=\mathbf{0 . 0 1}$. Images from $0.5 \mathrm{~ms}$ to $4.5 \mathrm{~ms}$ show randomly activated pixels due to noise-induced random detection of activation times before activation actually starts at $5.5 \mathrm{~ms}$. The red dot should hypothetically denote the start of depolarization, i.e. the location of pacing. The green arrow shows the spatial translation needed to correct for the center of the activation (green star), which otherwise might incorrectly affect velocities. Scale bar $=3 \mathrm{~mm}$.

patchy configuration that doesn't correlate well with the "real" underlying activation, mainly due to the arbitrarily small and large activation time differences between the neighboring pixels respectively. Surprisingly, only the AF illustrates the real activation pattern with this noise level, clearly separating the activated area from the resting medium as the wave propagates (Figure 16b). However, a correction is needed to restore the geometric center onto the starting position of the real depolarization. The random noise-induced detection of activated pixels before $t=5.5 \mathrm{~ms}$ didn't contribute to the evaluation of velocity, since the total activated area remained near 0 in the corresponding time series for several frames before the slopes take off and the real activation dominates (refer to Figure 13 for an illustration of these time series).

\subsubsection{Sensitivity to Heterogeneity in the Milieu of Propagation}

In the presence of random spatial (structural) or temporal heterogeneity, analytical strategies might return velocity values, which are not representative of the whole medium, an effect that 
inadvertently challenges the accuracy of the obtained result. Using simulated data, a gradual increase of spatial heterogeneity on the excitable medium is implemented and manifests itself by an increase in the severity of the contour lines' deformations (Figure 17a), creating therefore patchy configurations where the isochronal invaginations and the protrusions correspond to areas of slower and faster conduction respectively.

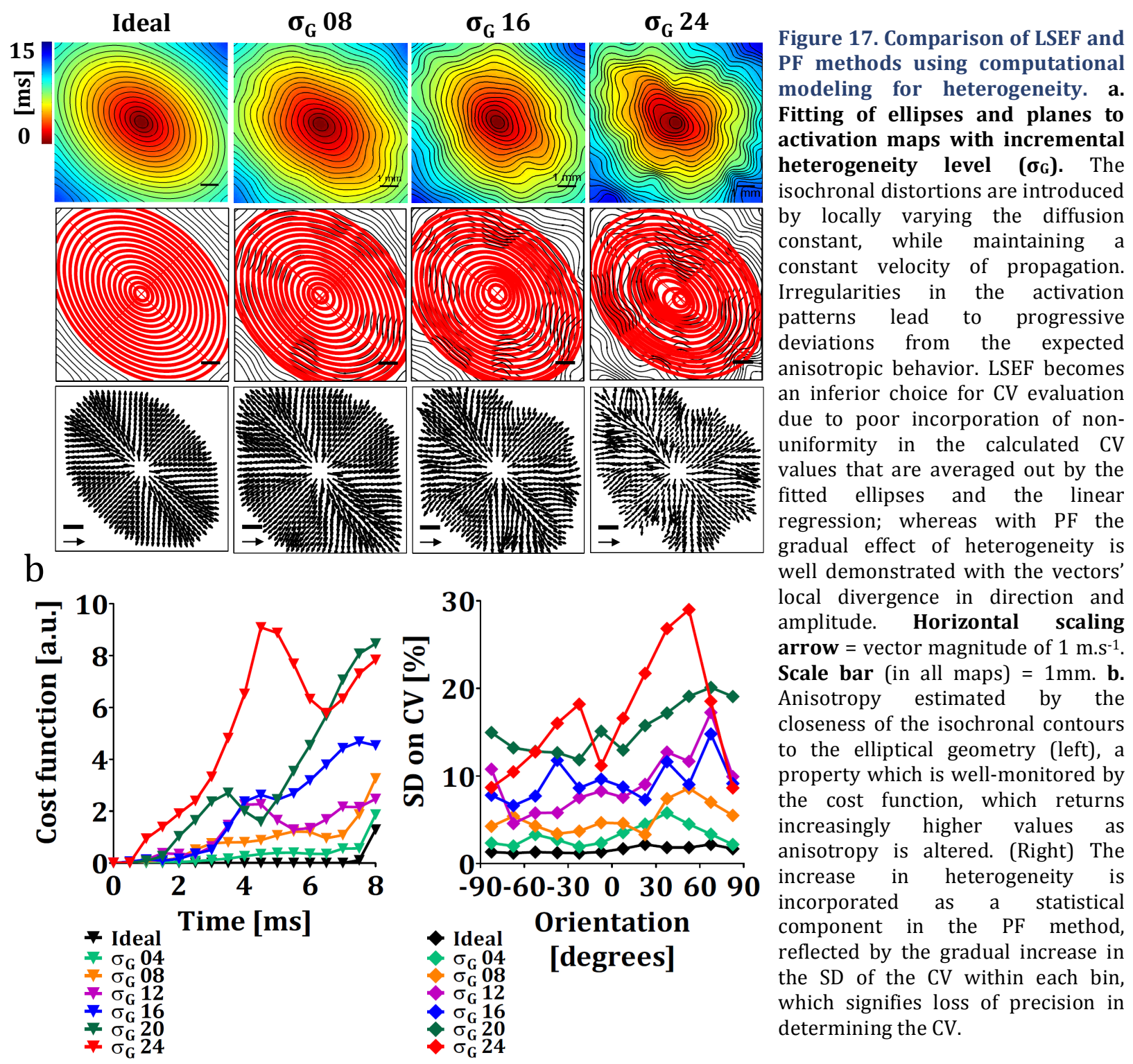

These simulations tried to mimic the activations observed for the $\triangle \mathrm{KPQ}$ hearts that were treated with a $\mathrm{Na}_{v} 1.5$-blocker. For low to moderate heterogeneity level (e.g. $\sigma_{\mathrm{G}} 12$ or below), the LSEF and AF seem to work rather well, compared to the PF. The velocity vs. sliding interval graph (Figure 18b) offers the possibility to detect the varying transitions in the estimation of velocity at constant time intervals but variable spatial distributions (the outward emanating wave from a point stimulation progressively increases in size as it moves away towards the boundaries). Each colored curve corresponds to one analytical method and each point is the outcome of evaluating the velocity at a particular zone of the map. As it can be seen from the graph on the left, the LSEF returns a $V_{\max }$ $12 \%$ less than the other two methods over the ROI that extends from the $4^{\text {th }}$ to the $8^{\text {th }}$ isochrone. 
Excluding this point, the remaining points don't show a variation that exceeds 7\%. It's also worth mentioning that for the same heterogeneity level, several realizations were simulated and analyzed accordingly, in principal to guarantee dependence of the curves on heterogeneity, rather than on a particular isochronal configuration. The graph, on the right in Figure 18b, reveals deviations of more than $20 \%$ among the mean velocities at different ROIs with maps embodying higher heterogeneity levels (e.g. $\sigma_{G} 24$ ). At this level, the curves do not show consistency among the

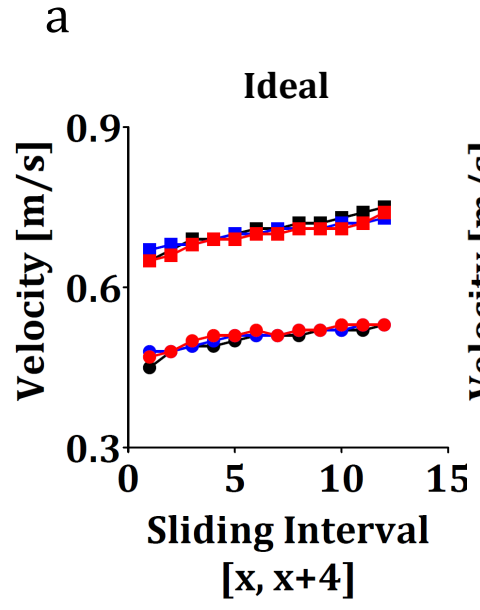

$\mathrm{b}$

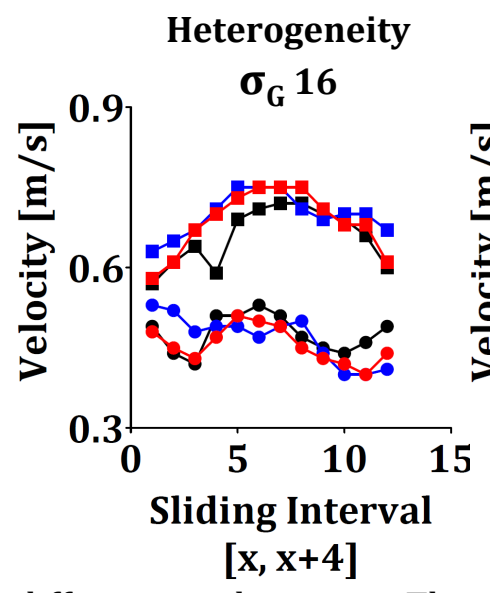

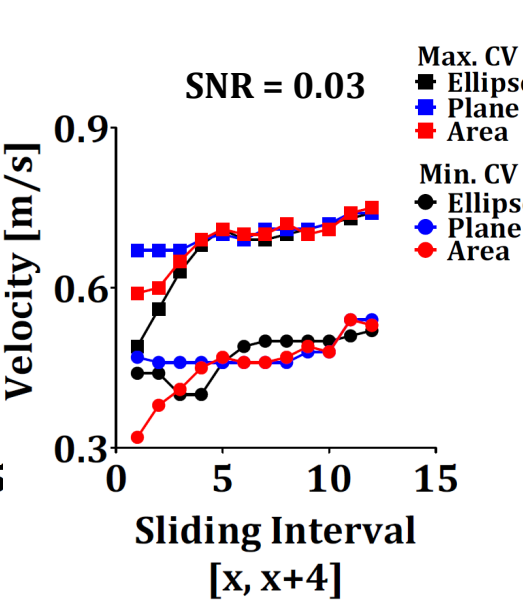

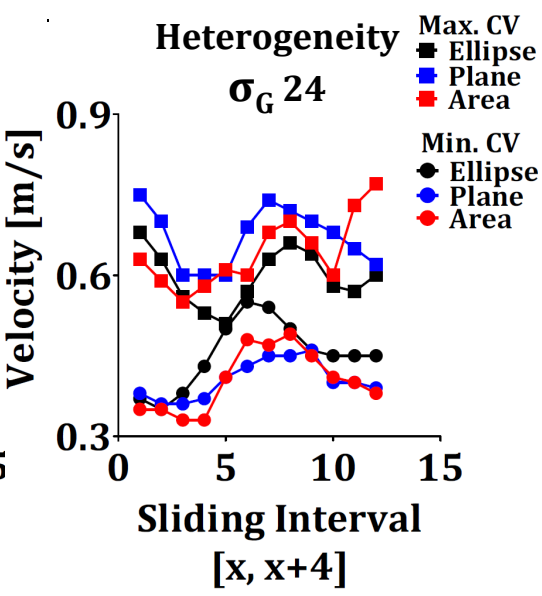

Figure 18. Plot of the maximal and minimal velocities vs. sliding interval. a. Methods correspondence with increasing noise. Each interval $[\mathrm{x}, \mathrm{x}+4]$ corresponds to one ring of inner circumference formed by the $x^{\text {th }}$ isochrone and outer circumference by the $(\mathrm{x}+4)^{\text {th }}$ isochrone. This ring is a well-defined ROI where the minimal and maximal CVs were calculated. By sliding this ring, curves that scan through the entire activation map are obtained, revealing the deviations from the ideal configuration observed as velocities are calculated by the three different methods. Evidently, all methods show robustness to noise with very comparable behavior (except for early isochrones) until complete misdetection of activation times at SNR $<0.03$ for LSEFP and PF (Figure 16). b. Methods correspondence with increasing heterogeneity. The observed fluctuations in velocity values reported by the methods are due to the continuous increase in heterogeneity level. Discernible divergence among the values is detected where anisotropy is largely disturbed (right). Square filled symbol: maximal $\mathrm{CV}$; Circular filled symbol: minimal CV; black: LSEF; blue: PF; red: $\mathrm{AF}$.

different realizations. The behavior is expected, where experimentally this could represent completely distinct substrates. Heterogeneity and uncertainty due to the associated error will ultimately influence the velocity measured and its significance. A global estimate of velocity will deviate from the local one, once the heterogeneity in the medium creates an activation complex enough to alter the morphology of the propagation pattern. Since only PF provides local calculations of velocity, small local heterogeneities are readily accessible for further analysis, where the mean velocity within the heterogeneous bin can be contrasted to the average velocity in the medium. To further explore this prevailing hypothesis, the effect of heterogeneity on the SD is plotted in Figure 17b. The PF tends to incorporate inhomogeneity into its statistics. Therefore the progressive increase in SD values within each bin, at almost all orientations, reflects the sensitivity of the method in picking up heterogeneities in the medium (Figure 17b, right). The LSEF and AF can't provide such a feature. Nevertheless, an indirect measure of heterogeneity can be provided as deviations of the isochrones from the elliptical geometry, which can be quantified using the cost function: as the heterogeneity strength progressively increases, the cost function faithfully mirrors this effect (Figure 17b, left). 


\subsection{Flecainide Proarrhythmia Scaled-Down in the $m d x$-Model}

Pharmacological management of clinical arrhythmias remains empiric at best, a trial-and-error process actualized on a case-by-case basis and guided by one's clinical acumen ${ }^{540,541 . ~ T h e ~}$ preeminent reason remains our deficiency in understanding the complex pathophysiological mechanisms that govern electrical cardiac rhythm disturbances ${ }^{542,543 .}$

In most cardiac cells, $\mathrm{Na}_{v} 1.5$ provides the ionic current required to initiate and sustain a propagating wave. Their nonlinear responses to changes of membrane potential allow them to switch between conducting and non-conducting states. These membrane proteins appear today more intricate than previously thought. The emergence of lethal diseases solely based on the possibility of existing flaws in their switching behavior puts into perspective our previous deficiency in appreciating the complexity of the protein. Genetic mutations, cardiac remodeling and drugs are famous modulators of Nav1.5 function. For instance, class I agents are among the most widely used antiarrhythmic drugs (AADs) ${ }^{478}$. They are further grouped into three different subclasses ${ }^{541,544}$ (the Vaughan Williams Classification) given their different interactions (kinetics, channel binding state... etc.) with their molecular target. The electrophysiological effects of $\mathrm{Na}_{v} 1.5$-blockers have generally been thought to be uniform, straightforward, hence well-understood478,479,511. Extrapolating cellular findings to a whole organ is now considered at best "ambitious". "Block of conduction at one site may be antiarrhythmic but become proarrhythmic at another site" 454 . Possibly... But, how far spatially are these two different sites exactly? What defines anti-/proarrhythmic activity in a multicellular network? How should we alter our experimental methodologies to improve the predictive outcome -with more certainty- of any means of treatment?

In the last two decades, multi-electrode arrays followed by optical mapping experiments of animal hearts showed that these drugs could produce divergent and sometimes contrasting effects in the multicellular tissue, as opposed to the clearly well defined effects in isolated cells ${ }^{36}, 545$. This paradoxical association between Flecainide (a Class IC Nav1.5 blocker) and its proarrhythmic responses in a multicellular network of cells is investigated in the next sets of experiments with cardiac substrates that have one common denominator: abnormalities in $\mathrm{Na}_{v} 1.5$. My approach includes observing the effects of targeting $\mathrm{Na}_{v} 1.5$ in the whole organ (mouse heart) with a drug (Flecainide) using concentrations within the therapeutic range $([1 \mu \mathrm{M}])$ and characterizing through whole heart ex-vivo optical mapping the spatial-temporal heterogeneity that could arise from pharmacologically modulating the function of this protein under pathological ( $m d x, \Delta \mathrm{KPQ}$ models) and normal (WT) conditions.

\subsection{1. (dF/dt) $\max$ and CV: Indexes of Nav1.5 Availability and Conductance?}

Two active factors affect the propagation of the cardiac impulse: The magnitude of the fast inward $\mathrm{I}_{\mathrm{Na}}$ and the time required to reach its peak ${ }^{99}$. A decrease in this current would be expected to decrease both the maximum rate of rise of the AP upstroke $(\mathrm{dF} / \mathrm{dt})_{\max }$ and the conduction velocity (CV). It has been assumed that the effects exerted by AADs, particularly the Class I drugs, on CV correlate well with their effects on $(\mathrm{dF} / \mathrm{dt})_{\max }$, because of their direct modification of $\mathrm{I}_{\mathrm{Na}}{ }^{92,} 362$. Nonetheless, it doesn't mean that the relationship governing changes between any of the three components should necessarily be linear $96,546,547$. The results presented in section 3.1 certainly show that under quasi-physiological conditions where anisotropy is preserved, the $m d x$ heart, which is a model deficient in Nav1.5, sustains an electrical activity of lower velocity than its WT counterpart does. Nevertheless further blocking of $\mathrm{I}_{\mathrm{Na}}$, through a commonly used AAD (Flecainide) does not compulsorily alter $\mathrm{CV}$ and $(\mathrm{dF} / \mathrm{dt})_{\max }$ in a predictable fashion in any of the substrates. 


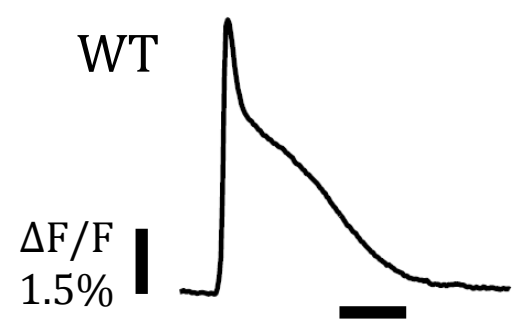

$20 \mathrm{~ms}$

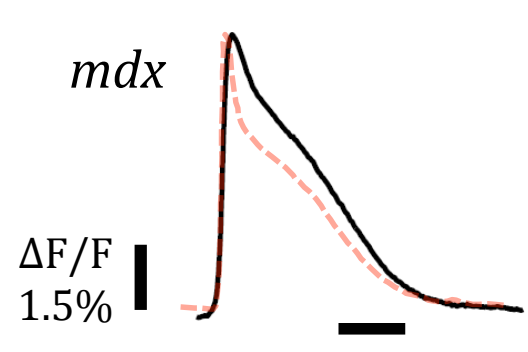

$20 \mathrm{~ms}$
Figure 19. Optical epicardial APs show morphological differences at control conditions. $m d x$ AP (right, black) when superimposed on WT (dotted, pink) shows a slightly shallower upstroke, a decreased notch and a higher plateau. Full recovery however is achieved at almost the same time point as the WT.

After processing and filtering, the conditioned optical signal is used for further analysis. Figure 19 above shows remarkable morphological differences between the optical APs (OAPs) recorded from the LV epicardium of the WT heart (left) and $m d x$ (black, right) under control conditions (i.e. no drug is added), stimulated with a $B C L=100 \mathrm{~ms}$. Prior to normalization, both signals were checked for equal fractional change $\left(\mathrm{FC}_{\%}=\Delta \mathrm{F} / \mathrm{F}\right)$ and amplitude. Normalizing a weaker signal to another one with larger amplitude could lead to erroneous prolongation of the earliest repolarization phases of OAP, an artifact that could cause the APD to "appear" prolonged at these levels. The $m d x$ AP is superimposed on a dotted representation of the same WT AP on the left for a direct comparison. The major findings can be summarized as follows: the less prominent notch is caused by the shortened phase 1 , which is preceded by a slightly depressed upstroke. The plateau phase therefore starts at more positive voltages in the $m d x$ than it does in the WT. The plateau phase in the mouse heart is normally short $(<15 \mathrm{~ms})$ due to the eminent $\mathrm{I}_{\mathrm{K}, \mathrm{t}}$ in these hearts that favors a fast early repolarization to more negative potentials and the kick-in of the delayed rectifier repolarizing currents brings the membrane potential back to the resting state ${ }^{501}$. In the $m d x$ heart, the AP appears prolonged in the early stages following the depolarization upstroke. However, recovery is fast enough to compensate for the previous delays in repolarization and brings the $m d x$ AP to resting potentials at almost the same time point as its WT counterpart.

Once activation times at all pixels are assigned, isochronal maps are generated. They describe the activation pattern of the electrical wave elicited at the LV free wall center, with a spatial and temporal resolution of $100 \mu \mathrm{m}$ and $0.5 \mathrm{~ms}$, respectively. Since the waves travel periodically $(10 \mathrm{~Hz})$ across the myocardium in all measurements, a time window can be selected within steady state conditions. In Figure 20a, the maps reproduce the effects detected previously concerning conduction slowing in the $m d x$ heart in all directions. After $t_{10}=10 \mathrm{~min}$ of Flecainide perfusion, isochrones are considerably tightened in both maps (Figure 20b). Qualitatively, differences in conduction become more challenging to observe between the two substrates. The appearance of the darker blue color in the $m d x$ map means that a longer time is needed for the wave to travel almost the same distance, suggesting a slower conduction in the $m d x$ heart. Despite overall decrease of conduction, an area of faster conduction emerges on the upper right corner of the WT map. This effect, previously thought to be an artifact due to boundary conditions, is reported in 6 out of 10 experiments, exclusively in WT group. 
Figure 20. Activation maps from WT and $\mathrm{mdx}$ LV free wall epicardium. a. At Control conditions (+0min Flecainide). Isochronal lines in the $m d x$ (right) are more closely spaced than WT, suggestive of conduction slowing. From the LV center, the electrical wave propagates toward the boundaries anisotropically. b. $\mathbf{t}_{10}=10 \mathrm{~min}$ Flecainide $[1 \mu \mathrm{M}]$. Activation maps of both hearts show further slowing of conduction, and isochronal tightening around the source of stimulation. The propagation remains anisotropic however its axis appears shifted counterclockwise in both maps compared to control conditions. In the upper right side of the WT map, an area of increased conduction emerges, through the increased spacing of isochrones in that region. Look-up-table: Activation time range in $\mathrm{ms}$ [0 20]. Scale bar $=0.5 \mathrm{~mm}$. Anatomical annotations: Ao $=$ Aorta, $\mathbf{A p}=$ Apex, $\mathbf{L}=$ Left, $\mathbf{R}=$ Right. $\mathbf{P}=$ Point stimulation. a

WT
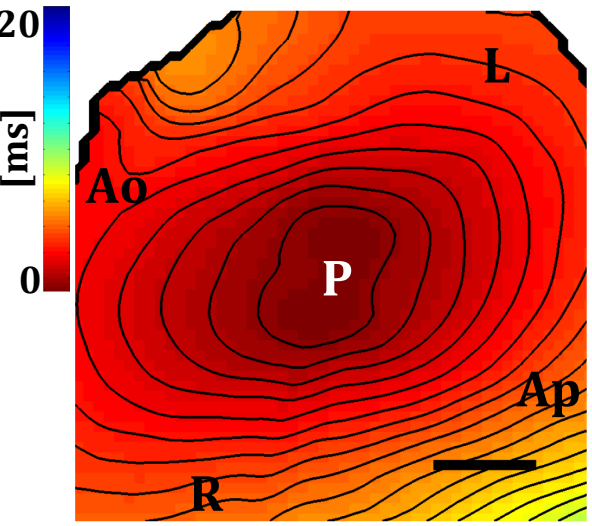

$m d x$

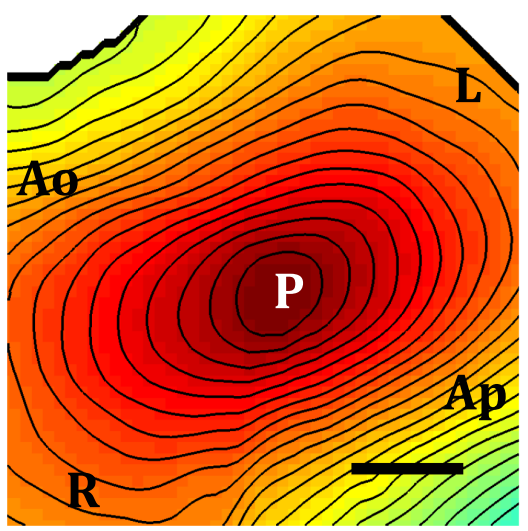

Control conditions: no treatment

b WT

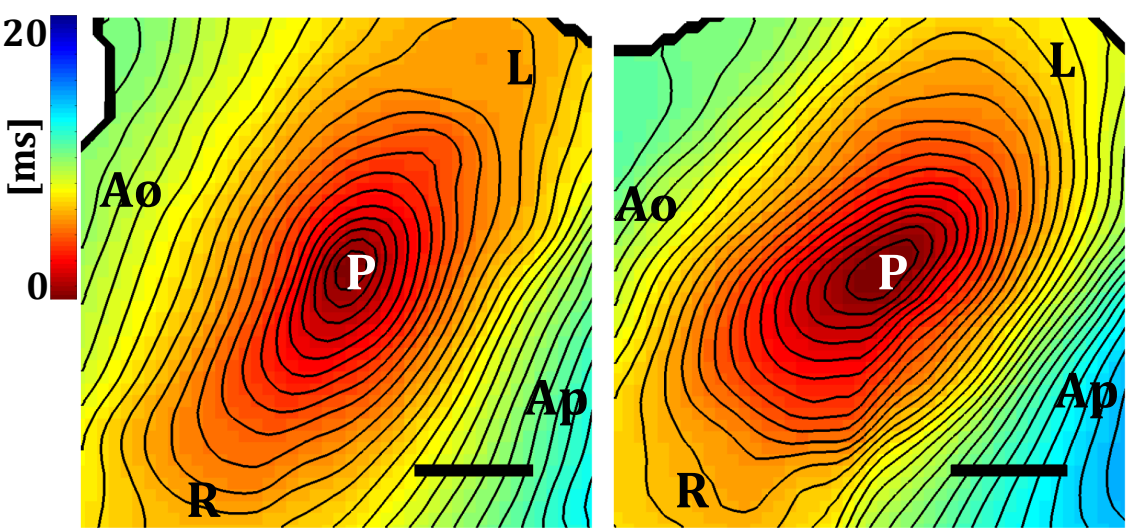

After 10 min treatment with Flecainide $(1 \mu \mathrm{M})$

From comparing the different analytical strategies used to evaluate CV in the previous subsection, the PF method was identified as the most sensitive in picking up CV fluctuations, due to its built-in capacity in evaluating velocity vectors locally. The aim of the current study is to characterize the effects of blocking Nav1.5 on the macroscopic component of propagation (i.e. CV) on one hand. On the other hand, we'd like to know whether CV continues to correlate well with the maximum upstroke rise of the APs $(\mathrm{dF} / \mathrm{dt})_{\max }$ in every direction, at all experimental time points of the treatment (i.e. at $0,+1,+3,+5,+7$ and +10 min). Using the PF method, CV dispersion maps were therefore produced within the ROI previously described ${ }^{\mathrm{x} v}$. In Figure 21a (upper, left), velocity values are distributed along the axes of maximum and minimum propagation, as depicted from their corresponding maps. The faster velocities in orange red appear in the WT map at both sides of the pacing point, with the lower velocities arranged perpendicularly. The same effect can be seen in the $m d x \mathrm{CV}$ dispersion map (Figure 21a, upper right), however with diminished velocities, as expected. After $t_{10}$ of drug perfusion, histograms (Figure 21b) reveal a shift of velocity distribution to the left, particularly for the WT heart, where the number of counts for the velocity values below $0.5 \mathrm{~m} . \mathrm{s}^{-1}$ increased significantly. The overall decrease in transversal velocities in this example is $\sim 26 \%$ and $\sim 34 \%$ for WT and $m d x$ respectively. While the velocities along the longitudinal direction is overall decreased in the $m d x$ by $\sim 35 \%$, only one side showed a decrease of $\sim 32 \%$, whereas an increase of

lxv Refer to Experimental Methods section 2.2.3 
$\sim 57 \%$ in velocity occurred on the diametrically opposite side. The vectors with higher velocity norms are depicted in the velocity histograms below (Figure 21b, left).

Four velocities -corresponding to mean velocities in each of the 4 different $15^{\circ}$ bins $(2$ bins located along the longitudinal axis and the 2 others along the transversal axis)- were extracted from each velocity dispersion map and included in bar graphs (Figure 22), in order to compare the changes in velocity for WT $(\mathrm{N}=20)^{\text {lxvi }}$ and $m d x(\mathrm{~N}=14)$ in the longitudinal (Figure 22, upper) and transversal (Figure 22, lower) directions as the treatment with Flecainide progresses. Statistical significance was calculated using the unpaired t-test. The major findings can be summarized as follows: Flecainide causes a slowing in conduction in both directions of propagation for both groups. For the WT group, the standard deviation (SD) increases to become twice as large at 7 and 10 min of treatment compared to the control value, particularly for the longitudinal velocities (Average $\mathrm{V}_{\max }=$ $0.75 \pm 0.11 \mathrm{~m} . \mathrm{s}^{-1}$ at control conditions, $\mathrm{V}_{\max }=0.53 \pm 0.22 \mathrm{~m} . \mathrm{s}^{-1}$ at $\mathrm{t}_{10}$, Figure 22 , upper). This increase in SD is probably due to the one-sided increase in $V_{\text {max }}$, thus enhancing the discrepancy in the values within the same group. Even when the velocity is overall decreased at $t_{10}$ compared to control, $V_{\max }$ reached its lowest attainable value on average at $5 \mathrm{~min}$ post treatment with $V_{\max }=0.49 \pm 0.12 \mathrm{~m} . \mathrm{s}^{-1}$ (Figure 22, upper). The minimal velocities in the WT group show less fluctuation in terms of amplitude and SD than the maximal velocities $\left(\mathrm{V}_{\min }=0.38 \pm 0.06 \mathrm{~m} \cdot \mathrm{s}^{-1}\right.$ at control conditions, $\mathrm{V}_{\min }=$ $0.23 \pm 0.09 \mathrm{~m} . \mathrm{s}^{-1}$ at $\mathrm{t}_{10}$, Figure 22, lower). The gray bars show a SD of $\sim 10 \%$ at all time points, suggesting a more homogeneous distribution of values within the $m d x$ group (Figure 22, upper and lower) with a progressive and continuous decrease to reach the lowest values at $t_{10}$ post treatment (Average $\mathrm{V}_{\max }=0.52 \pm 0.10 \mathrm{~m} . \mathrm{s}^{-1}, \mathrm{~V}_{\min }=0.26 \pm 0.04 \mathrm{~m} . \mathrm{s}^{-1}$ at control conditions; $\mathrm{V}_{\max }=0.33 \pm 0.08 \mathrm{~m} . \mathrm{s}^{-1}$, $\mathrm{V}_{\min }=0.18 \pm 0.04 \mathrm{~m} \cdot \mathrm{s}^{-1}$ at $\mathrm{t}_{10}$ ). With a $\mathrm{V}_{\min }$ value of only $5 \%$ less in the $m d x$, the increase in SD in the WT to $9 \%$ abolished the statistical significance of $V_{\min }$ at $t_{10}$ between the two groups (Figure 22, lower, last two bars).

Since $(\mathrm{dF} / \mathrm{dt})_{\max }$ is regarded as one of the most important factors determining propagation in the mammalian heart, the relationship between upstroke velocity and CV has been so widely accepted that several studies only offered measurements of $(\mathrm{dF} / \mathrm{dt})_{\max }$ as evidence for changes in $\mathrm{CV}^{99}$ at normal values of resting membrane potential $\left(\mathrm{V}_{\mathrm{rmp}} \sim-70 \mathrm{mV}\right)^{92}$. This proposed relationship became questionable in light of noticeable examples 548,549 , that $(\mathrm{dF} / \mathrm{dt})_{\max }$ has been further probed to whether it could actually be used as an index of proportionality to CV or availability of Nav1.5 at take-off potentials. The following results investigate the sort of CV dependency on $(\mathrm{dF} / \mathrm{dt})_{\max }$ under normal conditions, but also reexamine $(\mathrm{dF} / \mathrm{dt})_{\max }$ as a determinant of $\mathrm{CV}$ in conditions of Nav1.5 blocking, where measurements of $(\mathrm{dF} / \mathrm{dt})_{\max }$ appear to be of little predictive value with respect to CV.

\footnotetext{
lxvi For the WT group, the number of experiments is $n=10$. Since two velocity points were extracted along the longitudinal axis and two others along the transversal axis, the overall number of velocity points in one direction is $\mathrm{N}=20$ (used in the bar graphs). The same goes for the $m d x$ hearts where $n=7$, but $N=14$ was used to make the bar graphs.
} 
Figure 21. Velocity dispersion maps and histograms. a. Evaluation of velocities within ROI at $\mathbf{t}_{\mathbf{0}}$ and $\mathbf{t}_{\mathbf{1 0}}$ Flecainide. Velocities in the WT control map are distributed along the respective axes of wave propagation. The faster velocities (red orange) are almost perpendicularly located to the slower ones (blue). The same anisotropic distribution is seen for the $m d x$ (upper, right), with a lower velocity range. Treating the hearts with Flecainide lead to an overall decrease in velocity values in both groups. A focus of faster conduction (1.2 m.s $\left.{ }^{-1}\right)$ appeared one-sided along the longitudinal direction. Such a focus doesn't appear in the $m d x$, where CV monotonically decreased as Nav1.5 is blocked by the drug. The map at $\mathrm{t}_{10}$ shows a homogenous distribution of velocities (bottom, right). Scale bar $=0.5 \mathrm{~mm}$. Look-up table: $\mathbf{C V}\left[\begin{array}{ll}0 & 1.2\end{array}\right] \mathrm{in}$ $\mathrm{m} . \mathrm{s}^{-1}$. Annotations in figure: $\mathbf{A o}=$ Aorta, $\mathbf{A p}=$ Apex, $\mathbf{R}=$ Right, $\mathbf{L}=$ Left, $\mathbf{P}=$ Point stimulation. b. Histograms of velocities of the above dispersion maps. A uniform shift to the left is seen in both groups with Flecainide. The focus of faster conduction is represented by dispersed high velocity values in the WT, but not in the $m d x$.

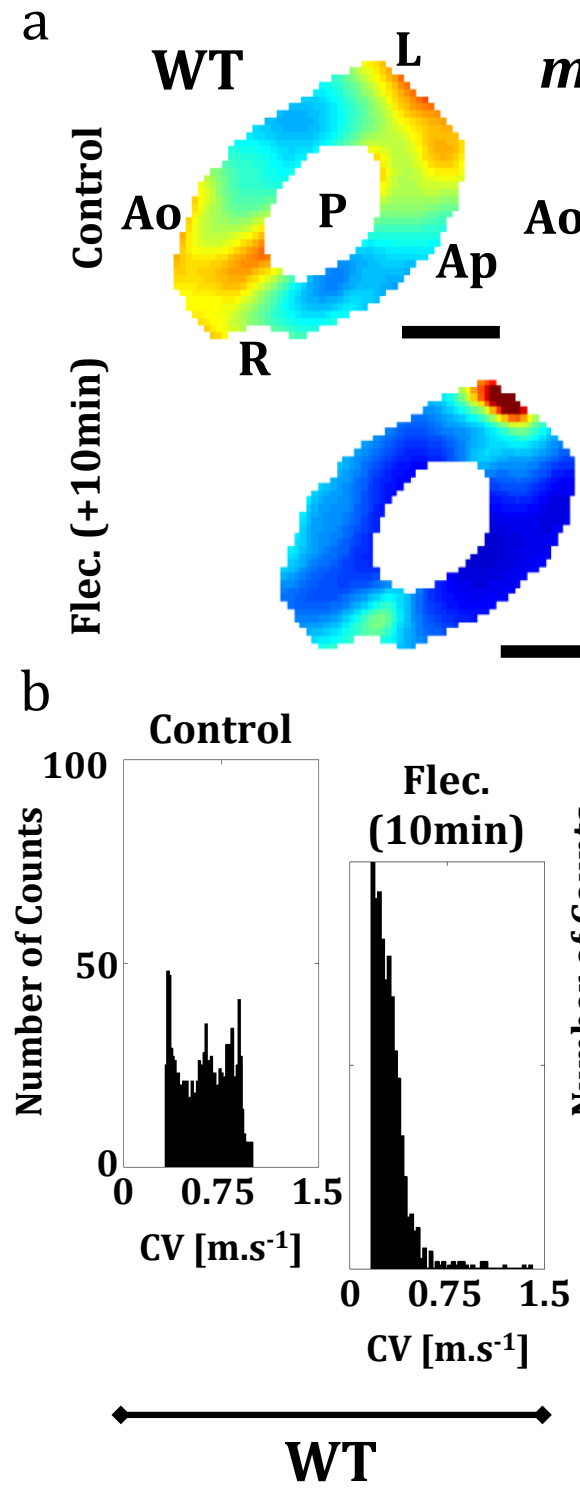

In Figure 23, $(\mathrm{dF} / \mathrm{dt})_{\max }$ is calculated for each pixel within the same ROI chosen for the velocity maps (Figure 21). This approach will identify at one-pixel resolution how the upstroke velocities react to the Nav1.5-blocking protocol and whether they mimic the CV fluctuations detected in the WT hearts. Under control conditions, both WT (Figure 23a, upper left) and $m d x$ (Figure 23b, upper left) upstroke velocities maps faithfully reproduce the CV patterns: the highest $(\mathrm{dF} / \mathrm{dt})_{\max }$ match well the $\mathrm{V}_{\max }$ distribution along the longitudinal axis, and the lowest $(\mathrm{dF} / \mathrm{dt})_{\max }$ along the transversal one. The $m d x$ heart that has a lower CV profile is also characterized with $(\mathrm{dF} / \mathrm{dt})_{\max }$ in the lower range. The steady loss of the original higher upstroke values with Flecainide perfusion is consistent with a homogeneous distribution of the drug to the tissue during measurements, as seen in both sequences (Figure 23a and b). The peculiar increase in CV seen in the WT heart at $t_{10}$ (Figure 21a, bottom left) however, doesn't seem to be present in the $(\mathrm{dF} / \mathrm{dt})_{\max }$ map (Figure 23a, bottom right). In this example, the location of fast propagation is indeed identified in an area of relatively higher $(\mathrm{dF} / \mathrm{dt})_{\max }$ compared to the rest of the ROI, however it is certainly not high enough to match the $\sim 60 \%$ increase in $\mathrm{CV}$ in this region; for the CV was measured to be $1.2 \mathrm{~m} . \mathrm{s}^{-1}$, which should 
correspond to a $(\mathrm{dF} / \mathrm{dt})_{\max }$ value near 1 a.u.ms ${ }^{-1}$ (based on previous measurements). By looking at upstroke velocities dispersion maps, we conclude on one hand that they correlate well with CV maps under control conditions and in the early stages of Nav1.5 blocking, where the OAP upstroke at each pixel becomes less steep. On the other hand, upstroke velocity maps obviously do not explain the outbreak of CV heterogeneity at later stages of Nav1.5 blocking. Therefore they do not predict CV distribution at the level of the tissue under these conditions.

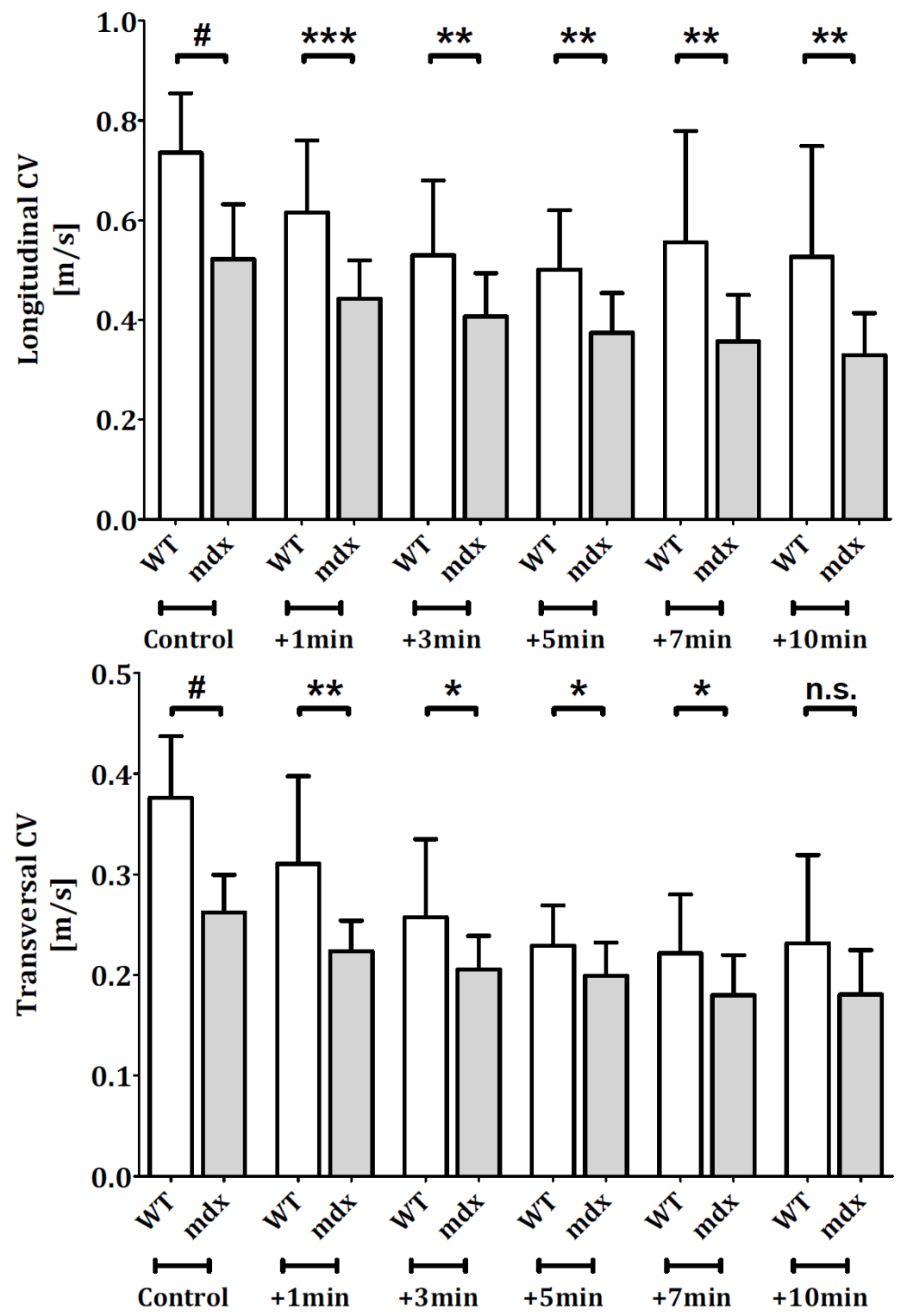

Figure 22. Bar graphs of $V_{\max }$ and $V_{\min }$ for WT $(\mathrm{N}=20)$ and $m d x$ $(\mathrm{N}=14)$ under control and different time points of Flecainide perfusion. (Upper) Decrease in $V_{\max }$ is consistent for both groups until later time points: A maximum decrease of $33 \%$ for WT (at $5 \mathrm{~min}$ ) vs. $36 \%$ decrease for $m d x$ (at $t_{10}$ ). The increase in $\mathrm{CV}$ at $+7 \mathrm{~min}$ and $+10 \mathrm{~min}$ of Flecainide perfusion is typically seen in 6 out of $10 \mathrm{WT}$ experiments. No such fluctuation was detected in the $m d x$ group. The increase in SD at $t_{7}$ and $t_{10}$ is relevant due to the enhanced scattering of $V_{\max }$ values in the WT. (Lower) Gradual decrease in $\mathbf{V}_{\text {min }}$ with Flecainide: $A$ total of $39 \%$ and $30.5 \%$ decrease in $V_{\text {min }}$ were measured for WT and $m d x$ respectively. The differences between velocities at all times is statistically significant (unpaired ttest), except for the $V_{\min }$ at $t_{10}$, where the difference between mean velocities $(<5 \%)$ is covered by the increase in SD of the WT group (9\%). Annotations in figure: \# $(p<$ $0.0001)$; $* * *(p<0.001) ; * *(p<0.01)$; * $(p<0.05)$; n.s. not significant.

In a similar fashion to the $\mathrm{CV},(\mathrm{dF} / \mathrm{dt})_{\max }$ data points were compared using bar graphs along the longitudinal (Figure 24a) and transversal (Figure 24b) axes. The results described in the dispersion maps correspond well with the decrease in $(\mathrm{dF} / \mathrm{dt})_{\max }$ in the bar graphs. In contrast to the $\mathrm{CV}$, $(\mathrm{dF} / \mathrm{dt})_{\max }$ don't show any effect related to an enhanced scattering between high and low values, hence no sudden increase in $(\mathrm{dF} / \mathrm{dt})_{\max }$ is found at later time points of Flecainide perfusion in any group. It's important to be reminded here that the values reported by the upstroke velocities do not represent the "absolute" membrane voltage values at the upstroke phase. Hence, its unit is not represented in V.s $\mathrm{s}^{-1}$ as in isolated cells studies, rather in a.u.ms ${ }^{-1}$ (a.u. stands for "arbitrary units"). 
Indeed the optical AP has been validated as a linear translation of the changes in membrane voltage 550 , however the data regarding upstroke velocities must be carefully represented. Another way of estimating the changes in $(\mathrm{dF} / \mathrm{dt})_{\max }$ during Nav1.5 blocking is to look at the "relative change" of $(\mathrm{dF} / \mathrm{dt})_{\max }$ at any time point of Flecainide treatment compared to the control conditions. This strategy is described in Figure 25a.

Figure 23. Dispersion maps within the ROI showing $(\mathrm{dF} / \mathrm{dt})_{\max }$ changes with Flecainide. Starting from a nicely anisotropic distribution of upstroke velocities (property of propagation, where not only the $\mathrm{CV}$ is anisotropic, but so are the upstroke velocities in the tissue), the drug progressively decreases the steepness of the upstroke at each pixel. a. Progression in the WT heart (from Control $\left(t_{0}\right)$ to $+1 \mathrm{~min}$ $\left(t_{1}\right),+5 \mathrm{~min}\left(t_{5}\right)$ and $+10 \mathrm{~min}$ $\left(t_{10}\right)$ drug perfusion). The red areas are gradually replaced by blue, suggesting a shift of the upstroke velocities towards lower values. At $t_{10}$, the distribution of $(\mathrm{dF} / \mathrm{dt})_{\max }$ remains homogenous, except for the upper side of the ROI, with a clear green coloration. The values of $(\mathrm{dF} / \mathrm{dt})_{\max }$ however do not explain the fast $\mathrm{CV}$ detected in that area. b. Progression in the mdx. The unremarkable changes in $(\mathrm{dF} / \mathrm{dt})_{\max }$ appear consistent with the changes in $\mathrm{CV}$ reported previously. Aside from the gradual decrease in the upstroke velocities throughout the ROI, the maps appear homogeneous and unremarkable. Scale bar = $0.5 \mathrm{~mm}$. Look-up table: $(\mathbf{d F} / \mathbf{d t})_{\max }$ in a.u.ms ${ }^{-1}=\left[\begin{array}{ll}0 & 0.8\end{array}\right]$. Annotations in figure: $\mathbf{A o}=$ Aorta, $\mathbf{A p}=$ Apex, $\mathbf{R}=$ Right, $\mathbf{L}=$ Left, $\mathbf{P}=$ Point stimulation.

a

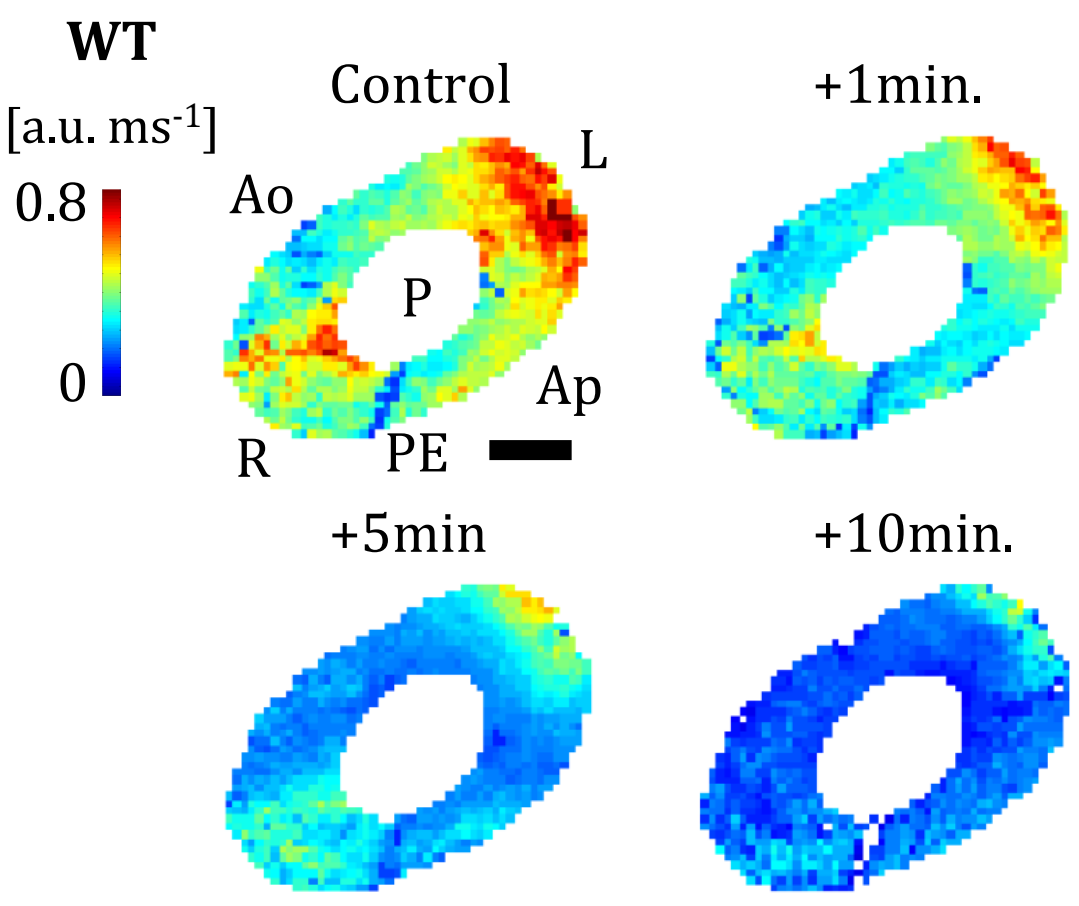

\section{$m d x$}

[a.u. $\mathrm{ms}^{-1}$ ]

\section{Control}

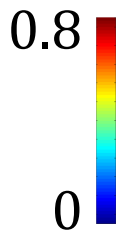

$\mathrm{L}$

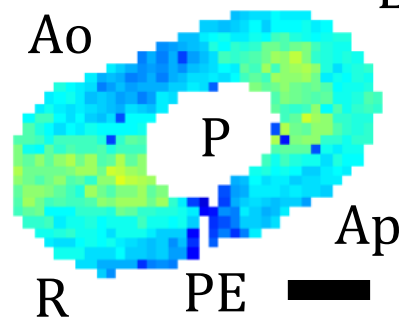

$+5 \mathrm{~min}$

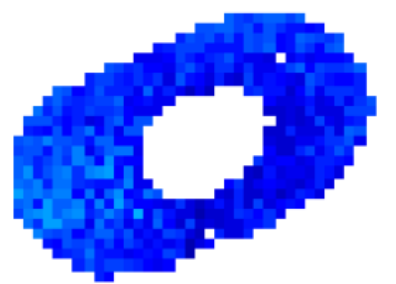

\section{$+1 \min$.}

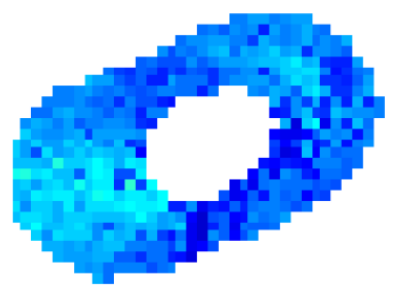

$+10 \mathrm{~min}$.

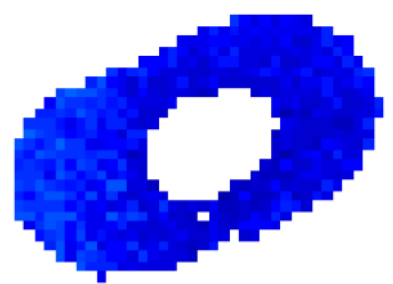




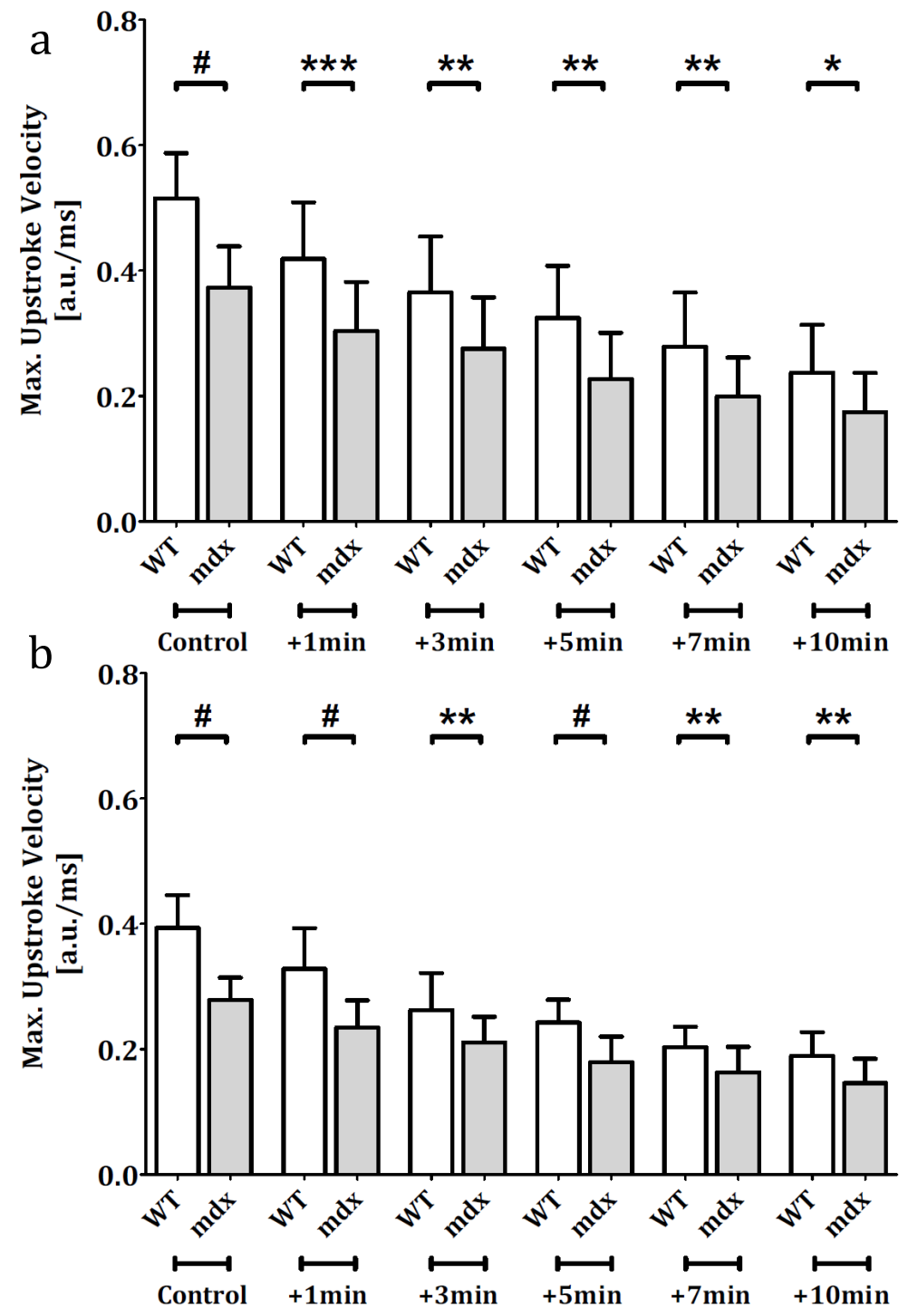

Figure 24. Bar graphs representing gradual changes in $(\mathrm{dF} / \mathrm{dt})_{\max }$ with Flecainide between WT $(\mathrm{N}=20)$ and $m d x(\mathrm{~N}=14)$. Progressive changes in upstroke velocities are visible at all time points of drug perfusion. a. Changes along the longitudinal direction. b. Changes along the transversal direction. As reported in cellular studies, blocking $\mathrm{Na}_{\mathrm{v}} 1.5$ will lead to decrease in the rate of rise of the AP. This effect is perfectly reproducible in the optical AP. At control conditions, the $m d x$ with initially lower $\mathrm{Na}_{\mathrm{v}} 1.5$ has a shallower $(\mathrm{dF} / \mathrm{dt})_{\max }$. These values, although describe the differences observed between WT and $m d x$ at each time point, they poorly represent the "relative" changes at each point from the initial starting point, and whether differences in the decay of $(\mathrm{dF} / \mathrm{dt})_{\max }$ exist between the two experimental groups. Annotations in figure: \# $(p<$ $0.0001)$; *** $(p<0.001) ; * *(p<0.01) ; *$ $(p<0.05)$; n.s. not significant.

Figure 25 shows the upstroke velocities at each time point normalized to the control value in percentage. At time $0,(\mathrm{dF} / \mathrm{dt})_{\max }$ is represented by the highest values recorded along the longitudinal and transversal directions for each preparation, corresponding to $100 \%$ in the plot. As the treatment progresses and the value of the upstroke velocity decreases, the following values represent a certain percentage of the control values. Each variable in the decay curve is the mean of percentage values of $(\mathrm{dF} / \mathrm{dt})_{\max }$ at a particular time point of the treatment protocol (bars $=\mathrm{SD}$ ). Figure 25a clearly demonstrates that despite the lower starting values of the upstroke velocities in the $m d x$ ventricular tissue, the fit of the one phase exponential decay is comparable to its WT counterpart. The red squares $(m d x)$ are almost superimposable over the blue squares (WT). The decay rates below (Figure 25b) are in agreement with the fit, with corresponding rate constants $\mathrm{K}_{\mathrm{WT}, \text { Long }}=0.21 \pm 0.07 \mathrm{~min}^{-1}$ and $\mathrm{K}_{m d x \text {,Long }}=0.19 \pm 0.09 \mathrm{~min}^{-1}$. The congruence in decay curves indicate that $(\mathrm{dF} / \mathrm{dt})_{\max }$ of the $m d x$ along the longitudinal direction has almost the same "sensitivity" as the WT's to Na 1.5 blocking. The plot on the right shows the decay of $(\mathrm{dF} / \mathrm{dt})_{\max }$ along the transversal direction with drug perfusion times. In contrast to the previous plot on the left, the WT curve shows a sharper decay almost twice as fast as the $m d x$ group, particularly seen in the early stages of the treatment. 
By looking at the WT values, $\mathrm{K}_{W T, T r a n s}$ is not only twice as large as $\mathrm{K}_{m d x \text {,Trans }}\left(0.34 \pm 0.05 \mathrm{~min}^{-1}\right.$ vs. $0.17 \pm 0.05 \mathrm{~min}^{-1}$ respectively) but also $\sim 13 \%$ larger than $\mathrm{K}_{\mathrm{WT}, \mathrm{Long}}$, which indicates that responses to $\mathrm{Na}_{v} 1.5$-blocking is not uniform in the cardiac tissue. This result could be attributed to alterations in active and passive electrical properties of the ventricular tissue, which play a role in excitation and conduction. The notably slower decay of $(\mathrm{dF} / \mathrm{dt})_{\max }$ in the $m d x$ substrate (compared to WT) along the transversal direction suggests an impaired ability of Flecainide to modify the excitability profile in this direction. This likely suggests that the loss Nav1.5 from the LM in the $m d x$ could play a role in preventing modulation of the channel pharmacologically, which translates as a form of drug resistance in the cardiac tissue.

A useful way of comparing how the alterations in $(\mathrm{dF} / \mathrm{dt})_{\max }$ and $\mathrm{CV}$ correlate is to plot them against each other (Figure 26) by dividing them into four groups according to their direction of propagation (longitudinal, transversal) and according to the two experimental groups (WT, N=20; $m d x, \mathrm{~N}=14$ ). Each plot contains $20 \mathrm{~V}_{\max }$ (square, blue) and $20 \mathrm{~V}_{\min }$ (circle, blue) values for the WT, as well as 14 $\mathrm{V}_{\max }$ (square, red) and $14 \mathrm{~V}_{\min }$ (circle, red) values for the $m d x$, each velocity with its corresponding $(\mathrm{dF} / \mathrm{dt})_{\text {max }}$. Starting with control conditions (upper left), it can be deduced that the relationship governing $(\mathrm{dF} / \mathrm{dt})_{\max }$ and $\mathrm{CV}$ is not exigently linear. The fact that data points within each ensemble occupy a particular zone of the plot suggests an overall interdependence mechanism among the groups, where fast CVs are associated with high $(\mathrm{dF} / \mathrm{dt})_{\max }$ and slower velocities with lower values of $(\mathrm{dF} / \mathrm{dt})_{\max }$ (as can be depicted from the distribution of blue squares and red circles for instance). Other areas of the control plot, however, could be harder to explain.
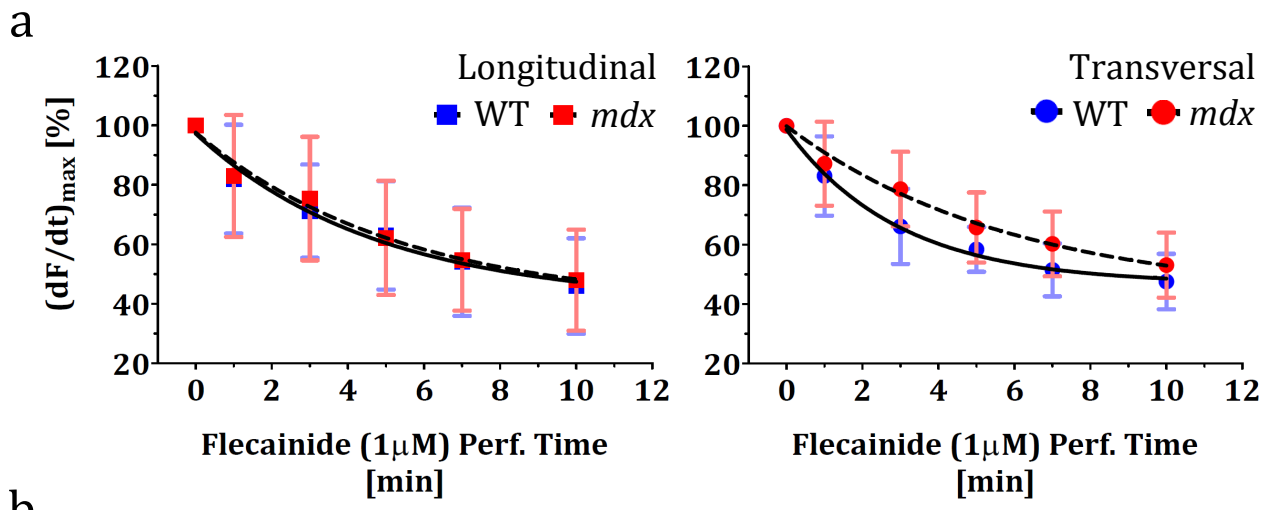

\begin{tabular}{|c|c|c|c|c|}
\hline \multirow{3}{*}{ نَّ } & \multirow{3}{*}{$\begin{array}{l}\text { WT } \\
\text { mdx }\end{array}$} & $\mathbf{K}\left[\mathrm{min}^{-1}\right]$ & $\boldsymbol{\tau}[\mathrm{min}]$ & $\mathbf{t}_{\mathbf{1} / \mathbf{2}}[\mathrm{min}]$ \\
\hline & & $0.21 \pm 0.07$ & $4.82 \pm 0.17$ & $3.34 \pm 0.22$ \\
\hline & & $0.19 \pm 0.09$ & $5.38 \pm 0.12$ & $3.72 \pm 0.15$ \\
\hline & \multirow{2}{*}{$\begin{array}{l}\text { WT } \\
m d x\end{array}$} & $0.34 \pm 0.05$ & $2.96 \pm 0.06$ & $2.05 \pm 0.08$ \\
\hline & & $0.17 \pm 0.05$ & $6.01 \pm 0.05$ & $4.18 \pm 0.10$ \\
\hline
\end{tabular}

Figure 25. Fractional (dF/dt) $\max$ decay with Flecainide perfusion. a. Plots show disparate responses to Nav1.5blocking, depending on the substrate and direction of propagation. The variables represent the mean of fractional values at a specific time point of the protocol. The bars represent the SD. The data are fitted using the one-phase decay equation: $\mathrm{y}(\mathrm{t})=\left(\mathrm{Y}_{0}-\mathrm{A}\right) \mathrm{e}^{(-\mathrm{t} / \tau)}+\mathrm{A}$, where $\tau$ is the time constant, $\mathrm{A}$ the plateau (i.e. $\mathrm{y}$ value at infinite times) and $\mathrm{Y}_{0}$ is the initial value at time 0 . (Left) $m d x$ and WT acquire similar decay curves with almost superimposable points, when (dF/dt)max is measured in the longitudinal direction. The plateaus reached are also within the same range of values where $A_{W T, L o n g}=40.3 \pm 8.2 \%$ and $A_{m d x, \text { Long }}=39.1 \pm 10.0 \%$. (Right) Transversely, $(\mathrm{dF} / \mathrm{dt})_{\max }$ curve's sharper decline in the WT at the early stages indicates the presence of a spatial component in modulating Nav1.5 blocking, which is deterred in the $m d x$. This could possibly be due to the loss of $\mathrm{Na}_{\mathrm{v}} 1.5$ on the LM in the $m d x$, which dampens down the sensitivity of the substrate to Flecaninde in this direction. Despite a faster decay in the early phases of treatment, the curves are expected to intersect at some point beyond $10 \mathrm{~min}$, if the tissues remain excitable, with a higher plateau value for the WT: AwT,Trans $=46.8 \pm 2.4 \%$ and $\mathrm{A}_{m d x, T r a n s}=41.9 \pm 9.1 \%$. b. Table of the decay parameters for both experimental groups, in the two directions. $\mathrm{K}=\mathrm{Rate}$ constant $\left[\mathrm{min}^{-1}\right], \tau=$ Time constant $[\mathrm{min}]=\mathrm{K}^{-1}, \mathrm{t}_{1 / 2}=$ Half-life $[\mathrm{min}]$. 


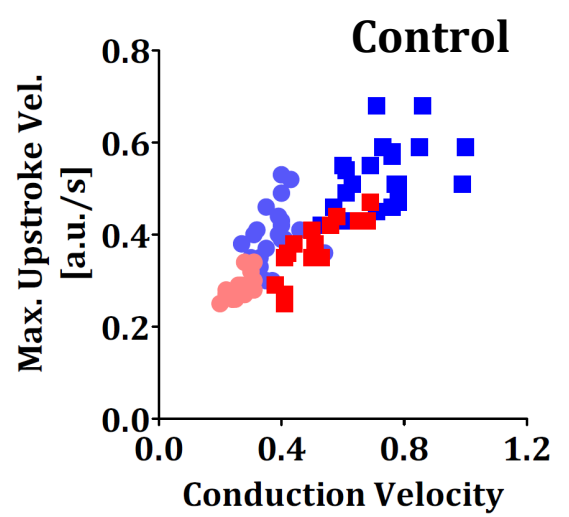

[m/s]

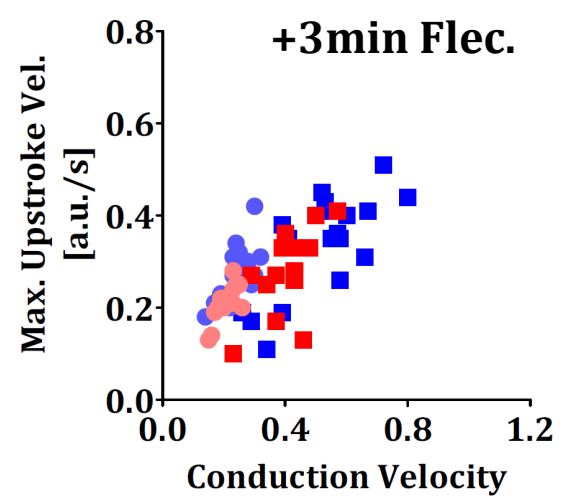

[m/s]

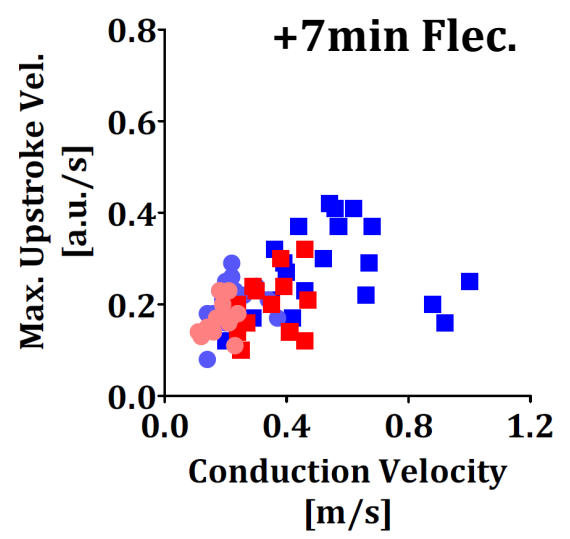

Long. Locations - WT

Trans. Locations - WT
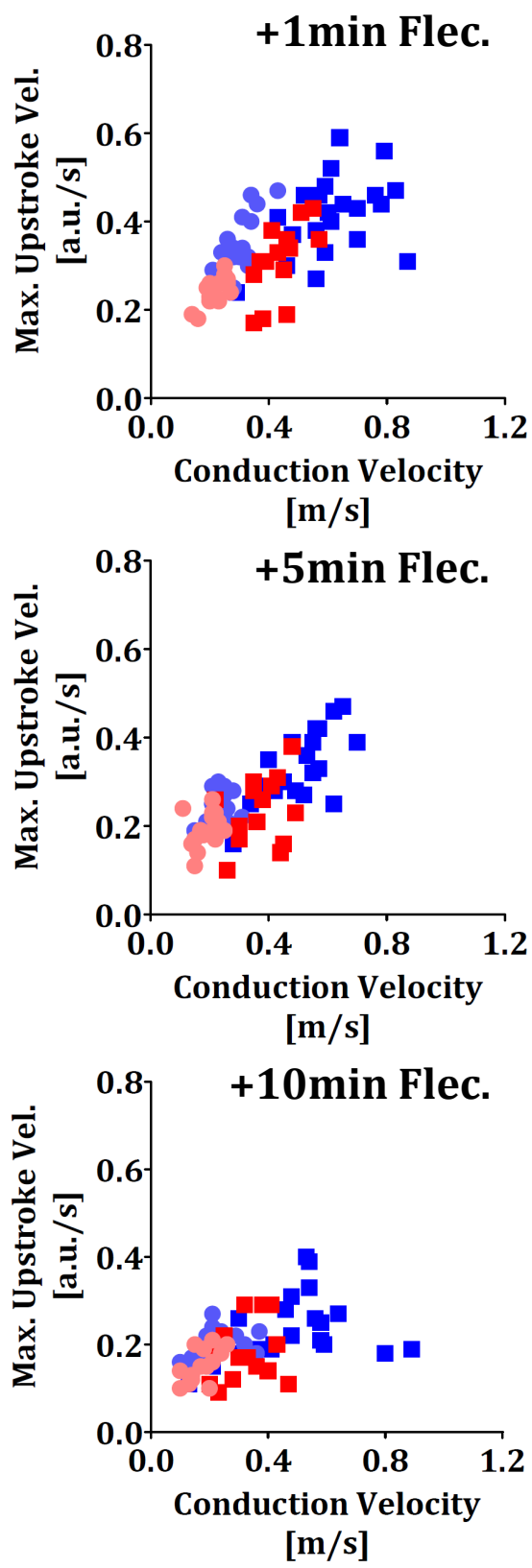

Long. Locations $-\boldsymbol{m d x}$

Trans. Locations $-\boldsymbol{m d x}$

Figure 26. (dF/dt $)_{\max }$ vs. CV correlation plots at different times of Flecainide $(1 \mu \mathrm{M})$ perfusion protocol. The clustering of data points in specific zones with relatively little overlapping (control) suggests a dependency between the two parameters of interest, even though no linearity can be deduced. At +1 min, data points show a shift to the left lower corner of the plot, which means that for all points, Nav1.5-blocking caused a decrease in both $(\mathrm{dF} / \mathrm{dt})_{\max }$ and $\mathrm{CV}$. The widened scattering simultaneously in all groups can be attributed to the general state of the system in transient phases (also seen at $+3 \mathrm{~min}$ ) between a control drug-free condition to a state of steady Nav1.5blocking (at $+5 \mathrm{~min})$. At $5 \mathrm{~min}$, the points occupy the lower left side of the plot, where the lower scattering is indicative that a new steady state is achieved in the presence of Flecainide. Beyond $5 \mathrm{~min}$ of treatment, the previously described faster CV foci kick-in and induce a widening of the zone occupied by the WT longitudinal data points, where fast CVs correlate with lower $(\mathrm{dF} / \mathrm{dt})_{\max }$ values. At this point, it's obvious that heterogeneity in propagation occurs mainly along the longitudinal direction, where increases in $V_{\text {max }}$ no longer correlate with $(\mathrm{dF} / \mathrm{dt})_{\max }$. The transverse direction appears to be independent of such effects.

The highest velocity ranges (blue squares, $\mathrm{WT} \mathrm{V}_{\max }$ ) are interspersed in the upper right side of the plot. Points that differ in less than $2 \%$ in the $(\mathrm{dF} / \mathrm{dt})_{\max }$ are associated with velocity values almost $40 \%$ in difference. For more than 6 data points, the red squares (which symbolize $V_{\max }$ in $m d x$ ) are almost horizontally aligned, which means that a steady $(\mathrm{dF} / \mathrm{dt})_{\max }$ value correlates with a whole range velocities (span $\sim 20 \%$ ). Similarly, the distribution of blue circles (i.e. $V_{\min }$ in WT) is almost vertical for more than 8 data points, which suggests that the same CV can be determined by a range of $(\mathrm{dF} / \mathrm{dt})_{\max }$ values. As shown in the remaining panels of Figure 26, as Flecainide blocks further $\mathrm{Na}_{V} 1.5$, the data points shift to the lower left corner of the plot, indicative of an overall decrease in $\mathrm{CV}$ and $(\mathrm{dF} / \mathrm{dt})_{\max }$. The data points within each group appear to scatter as they decrease. Since this 
scattering is occurring in all groups, it means that it's independent of the direction of propagation or the substrate at hand; rather it depends on the overall state of the system (cardiac tissue) being acutely exposed to a $\mathrm{Na}_{\mathrm{v}} 1.5$ blocking process. A new steady state is achieved at $5 \mathrm{~min}$, where the data points occupy the lower left region of the plot, indicative of a comprehensive decrease in propagation velocity and $(\mathrm{dF} / \mathrm{dt})_{\max }$. Beyond $5 \mathrm{~min}$, all groups appear in clusters of points particularly obvious in the $\mathrm{V}_{\max }$ (WT). If an imaginary line is drawn parallel to the $\mathrm{x}$-axis $(\mathrm{y}=0.2)$, blue squares could share the same $(\mathrm{dF} / \mathrm{dt})_{\max }=0.2$, but could acquire velocities varying between $0.4 \mathrm{~m} . \mathrm{s}^{-1}$ and $0.8 \mathrm{~m} . \mathrm{s}^{-1}$. In global terms, $(\mathrm{dF} / \mathrm{dt})_{\max }$ dispersion maps (Figure 23 ) and CV dispersion maps (Figure 21) appear reciprocal and interdependent. In local terms, this relationship is misleading since point selection (with some spatial preferences along fiber directions) reveals that the predictive potential of $(\mathrm{dF} / \mathrm{dt})_{\max }$ is quantitatively weak. This becomes more obvious when prediction completely fails with the onset of heterogeneous elements in the propagating medium (Figure $21 \mathrm{a}$, lower left panel) that increase $\mathrm{V}_{\max }$, along with a simultaneous decrease in $(\mathrm{dF} / \mathrm{dt})_{\max }$ (Figure 23a, lower right panel). In other words, the adopted rationale behind the creation of Nav1.5 blocking agents was in principle based on the outcomes of isolated single cell studies, which in light of these results is no longer justified. Henceforth, the conjectures of these studies need to be reevaluated, taking into account the behavior of the drug in a multicellular network.

Cell size 204 , geometry551 and intercellular coupling204 are all well-defined, structural determinants of anisotropy. However, anisotropy is not a static entity, it is subjected to modifications by nonstructural (functional) parameters such as frequency of stimulation ${ }^{552}$. Factors that alter CV in a direction dependent manner are expected to change the AR. In the results presented in the section 3.1, the change in AR between WT and $m d x$ is not statistically significant. To recall, CV was evaluated using three different methods, and the decrease in both $V_{\max }$ and $V_{\min }$ is comparable secondary to the loss of $\mathrm{Na}_{v} 1.5$ in the $m d x$ heart (Figure 11d, Figure 12d, Figure 13d). However, with addition of Flecainide, $\mathrm{V}_{\min }$ showed a steady decrease (in $m d x$ and WT), whereas $\mathrm{V}_{\max }$ started to increase beyond 5 min of treatment in the WT group only. In Figure 27a, the AR bar graphs show that under control conditions till 3 min of treatment, both velocities were changing in a comparable fashion in both groups, without altering the $\mathrm{AR}$ (At $\mathrm{t}_{0}, \mathrm{AR}_{\mathrm{wT}}=1.9 \pm 0.3$ and $\mathrm{AR}_{m d x}=2.0 \pm 0.3, p$-value=0.75). Beyond $5 \mathrm{~min}$, the increase in $\mathrm{V}_{\max }$ with the steady decrease in $\mathrm{V}_{\min }$ (Figure 22) leads to an increase in the $A R$ of the WT group only (Figure 27). Since $V_{\max }$ increase always occurs one-sidedly (Figure 21a, lower left), the same WT preparation shows a high and regular value of AR, leading to a simultaneous increase in the SD (at $t_{7}, \mathrm{AR}_{W T}=2.4 \pm 0.5$; at $t_{10}, \mathrm{AR}_{W T}=2.2 \pm 0.7$ ). Since CV wasn't subjected to heterogeneity in the $m d x$, the AR remains unchanged within the group over the whole period of treatment (at $\mathrm{t}_{7}, \mathrm{AR}_{m d x}=1.9 \pm 0.3$; at $\mathrm{t}_{10}, \mathrm{AR}_{m d x}=1.8 \pm 0.3$ ).

In order to estimate the propagation "speed" in the heart at later times of treatment without stumbling over the heterogeneity picked up by $V_{\max }$, we measured the global time $\left(\Delta \mathrm{t}_{\text {act }}\right)$ it took the wave to cover the LV epicardium from the point of stimulation to the boundaries. $\Delta t_{\text {act }}$ is a broad measure of time, hence less likely to be affected by the heterogeneity in local conduction, since the propagation at both sides of the pacing will average out in time. Bar graphs (Figure 27b) of $\Delta \mathrm{t}_{\text {act }}$ show that under control conditions significantly more time was needed for the wave to cover the LV in the $m d x$ heart $(\mathrm{n}=7)$ than in the WT heart $(\mathrm{n}=10)$ with $\Delta \mathrm{t}_{\text {act, } m d x}=12.0 \pm 1.6 \mathrm{~ms}$ and $\Delta \mathrm{t}_{\text {act,WT }}$ $=8.9 \pm 0.8 \mathrm{~ms}$ respectively ( $p$-value $<0.0001)$. At $\mathrm{t}_{10}$ of Flecainide perfusion, an increase of $\sim 107 \%$ in time was achieved in the WT heart vs. an increase of only $56 \%$ in the $m d x$, leading to an almost similar time window needed for the depolarization wave to cover both epicardia $\left(\Delta t_{\text {act, }}{ }_{T}\right.$ $=18.5 \pm 3.2 \mathrm{~ms}$ vs. $\Delta \mathrm{t}_{\mathrm{act}, m d x}=18.8 \pm 3.1 \mathrm{~ms}, p$-value $\left.=0.88\right)$. The overall decrease in $(\mathrm{dF} / \mathrm{dt})_{\max }$ at $\mathrm{t}_{10}$ of Flecainide was also estimated in percentage value along both directions (Figure 27c) in the $m d x$ $(\mathrm{N}=14)$ and WT $(\mathrm{N}=20)$. As expected from the exponential graph (Figure 25), the decrease in $(\mathrm{dF} / \mathrm{dt})_{\max }$ shows a significant difference between the groups exclusively along the transversal 
direction, with $52.3 \pm 10.1 \%$ and $39.4 \pm 6.2 \%$ for WT and $m d x$ respectively ( $p$-value<0.001). In the longitudinal direction, the decrease in $(\mathrm{dF} / \mathrm{dt})_{\max }$ was comparable between the groups with $51.8 \pm 16.9 \%$ decrease in the WT and $44.3 \pm 9.5 \%$ in the $m d x$ ( $p$-value=0.15). Interestingly in the WT heart, the overall percentile changes in $(\mathrm{dF} / \mathrm{dt})_{\max }$ at $\mathrm{t}_{10}$ are analogous regardless of fiber direction, despite the initially faster decay along one of the directions (Figure 25a and b). These results are at
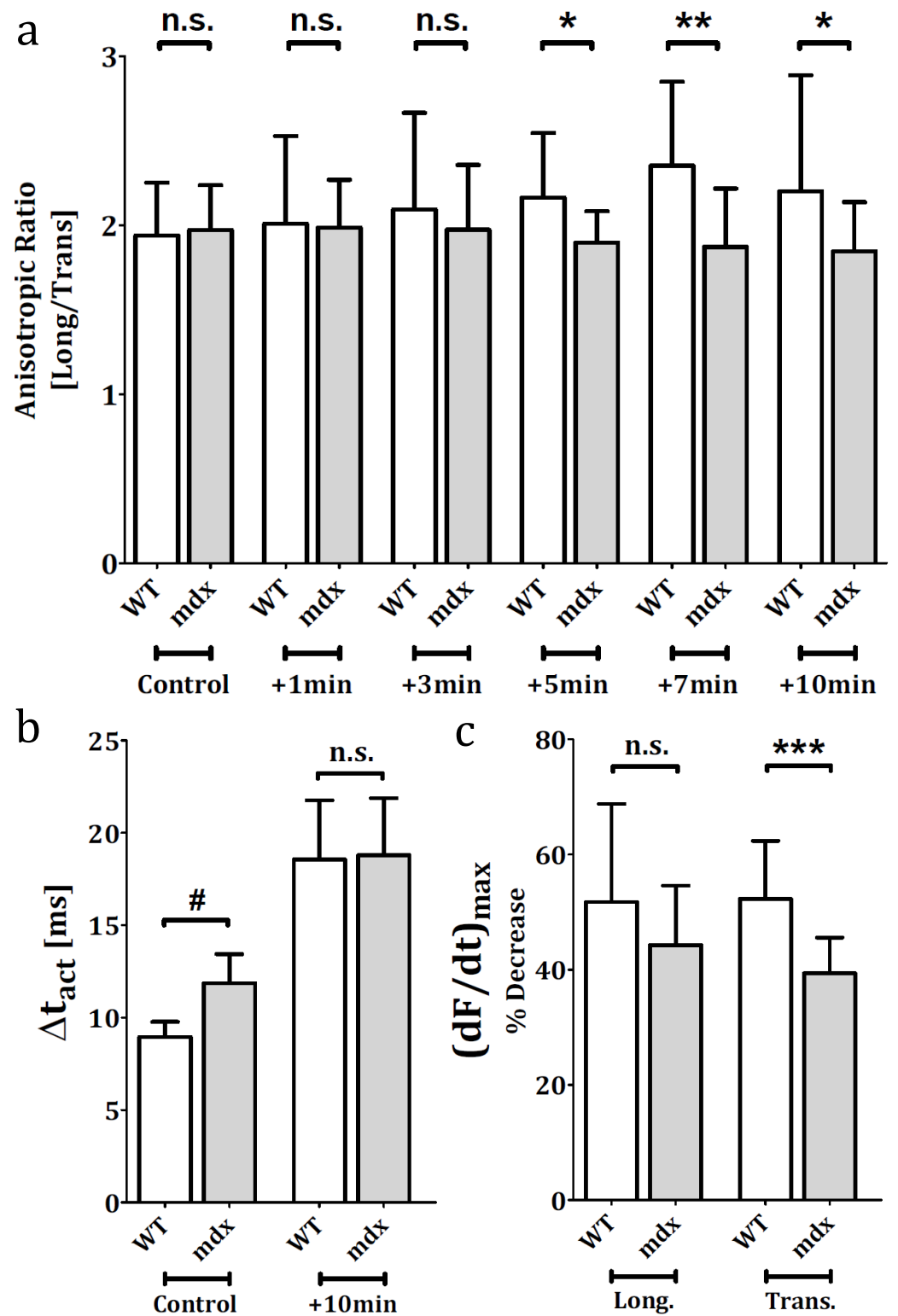

Figure 27. Bar graphs show changes in $A R$, the increase in $\Delta t_{\text {act }}$ and the total $\%$-decrease in $(\mathrm{dF} / \mathrm{dt})_{\max }$ at $\mathrm{t}_{10}$ of Flecainide perfusion for $m d x$ and WT hearts. a. Apparent increase in AR due to heterogeneity in conduction properties. With the increase in $V_{\max }$ and the steady decrease in $V_{\min }$ in the WT heart with Flecainide, the ratio of velocities is expected to increase in the WT. It remains unchanged in the $m d x$ increasing the differences in AR between the two groups beyond $5 \mathrm{~min}$. b. A total increase of $107 \%$ in $\Delta t_{\text {act,WT }}$ vs. a moderate increase in $\Delta t_{\text {act, } m d x}(56 \%)$ abolishes the difference in global depolarization time between the two groups at $t_{10}$ Flecainide. c. Bar graphs show that the total percentage decrease in $(\mathrm{dF} / \mathrm{dt})_{\max }$ between $\mathrm{t}_{0}$ and $\mathrm{t}_{10}$ of Flecainide along the longitudinal direction is comparable between WT $(51.8 \pm 16.9 \%)$ and $m d x(44.3 \pm 9.5 \%)$. This difference becomes more significant along the transversal direction with $52.3 \pm 10.1 \%(\mathrm{WT})$ and $39.4 \pm 6.2 \%(m d x)$. Annotations in figure: \# $(p<0.0001)$; *** $(p<0.001)$; ** $(p<0.01) ; *(p<0.05)$; n.s. not significant.

odds with the ones published by Iida et al. (1996), where Flecainide $(0.1-1 \mu \mathrm{M})$ appears to decrease the maximum upstroke velocity in the rabbit heart preferentially along the longitudinal direction ${ }^{553}$. The reason behind this discrepancy might lie in the methodology at the time by using bipolar extracellular electrodes to examine CV, and the inability to sufficiently resolve the cardiac tissue spatially and temporally, as offered nowadays by optical mapping.

\subsubsection{Symmetry Breaking: A Proarrhythmic Mechanism of Flecainide}

Regional differences in action potential duration (APD) contribute to spatial non-homogeneities in the three dimensional structure of the ventricular myocardium. Functional electrical asymmetry of 
the cardiac AP and dispersion of repolarization, often enhanced by intrinsic heterogeneities of the tissue electrical properties, can facilitate unidirectional block ${ }^{407}$ in the absence of conduction changes or an anatomical obstacle, solely based on temporal differences in recovery of excitability ${ }^{477}$. Experimental studies conducted on particularly high arrhythmogenic substrates ${ }^{264}$, such as Long QT- and Brugada syndromes, have reported significant dispersion in recovery of excitability. While differences in APD have shown to naturally exist across the heart ${ }^{262}$ (e.g. the apico-basal or transmural epicardium-endocardium axes), these gradual transitions are improbable to form substrates for arrhythmia under normal conditions. Distinct repolarization times are likely to become arrhythmogenic when their corresponding locations are "adjacent enough"554. Most of the heterogeneities in repolarization have been characterized in the time domain, whereas knowledge concerning the spatial extent of these APD gradients is relatively less certain ${ }^{254}$. The increasing resolution provided by optical mapping techniques555, in combination with computational simulations 270,556 , currently allows for a more precise analysis of the spatialtemporal extent of repolarization heterogeneity in the cardiac tissue and the elucidation of biophysical mechanisms possibly involved in promoting reentry.

APD dispersion maps are typically used to characterize spatial dispersion of repolarization (SDR) 550 . Although intrinsic cellular heterogeneity has been well characterized in isolated cells, taken from distinct areas of the heart 266,402 , electrotonic interactions are also known to modulate repolarization ${ }^{257}$ over large spatial extents ${ }^{402,557}$. The latter may have a relatively considerable impact on small size hearts, where functional dispersion of repolarization was found to be diminished 556 . The latter might render small animals (e.g. mouse) less favorable models for the investigation of APD dispersion in arrhythmogenic substrates compared to larger animals (e.g. rabbit, pig). At any rate, the cellular basis of ventricular wall heterogeneity is in principle based on the presence of three distinct types of cells that differ with respect to their early and late repolarization phases ${ }^{402}$. The response of each type of cells to AADs is therefore expected to vary, henceforth widens the underlying electrophysiological differences in the myocardium, a plausible mechanism into the proarrhythmic effects of AADs. As for activation times, APD from the optical signal can be calculated within each pixel and APD dispersion maps can be used to elucidate simultaneously temporal gradients in repolarization and their spatial extent in the region of interest.

In the next sets of results, we report how Nav1.5 blocking with Flecainide using clinically valid concentrations can produce opposite effects on APD-and repolarization-within the same population of cells (LV free wall epicardial cells) in the murine heart, under anisotropic conduction spread and steady-state entrainment conditions. We describe the temporal gradient and the spatial extent of such an effect, the repolarization inhomogeneity as it occurred consistently and exclusively in the WT group under the current experimental conditions. Interestingly, the $m d x$ group with lower available Nav1.5 channels appeared to be resistant to the drug-induced heterogeneity in APD. Following the presented arguments above, a substantial SDR has hardly been expected to occur in small sized hearts, not to mention the exclusivity of the symmetry breaking effect to the normal hearts sparing the hearts with initially lower $\mathrm{Na}_{v} 1.5$ channels. This effect hasn't been reported before in murine hearts, nor has the response of the dystrophic substrate to Flecainide been characterized. The molecular mechanism behind this observation remains to be fully elucidated; however it could provide insights into the electrophysiological process underlying why Flecainide is among the most arrhythmogenic and proarrhythmic of antiarrhythmic agents $483,492,558,559$. 
a
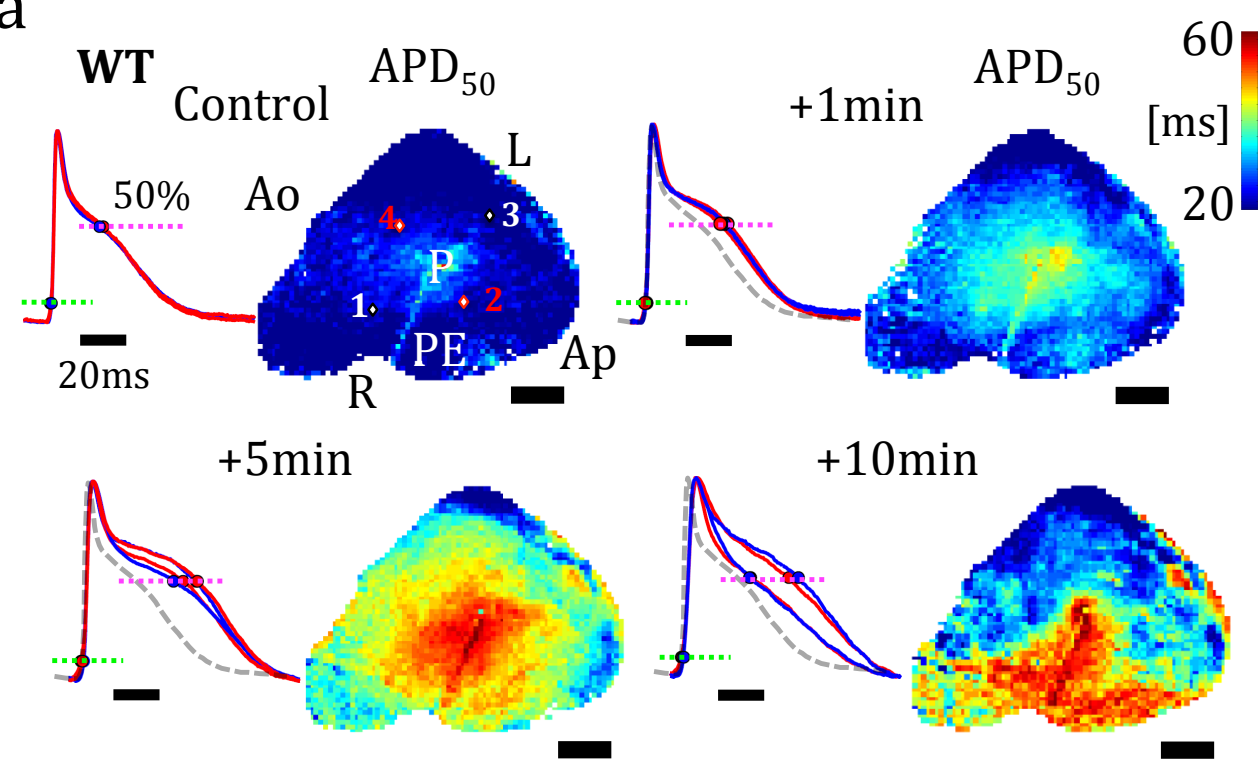

b
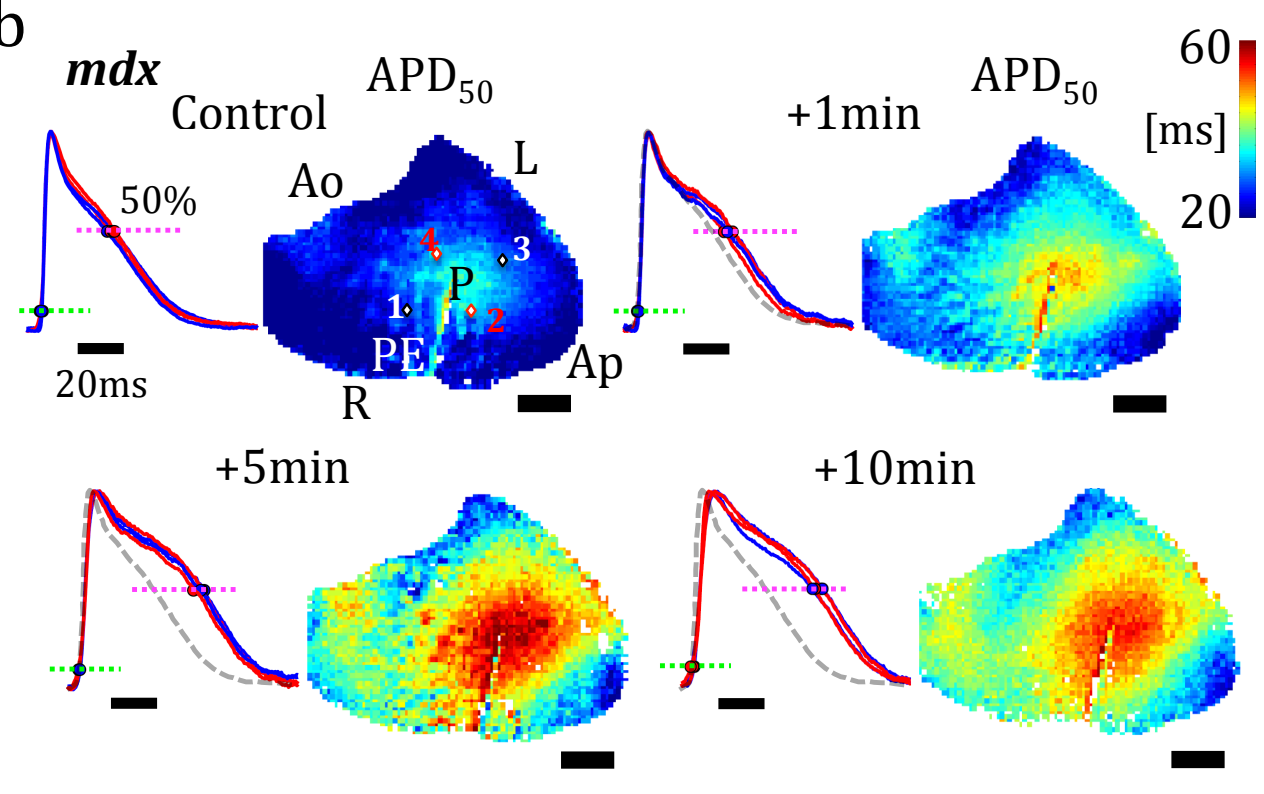

Figure 28. APs and APD 50 dispersion maps for WT (a) and $m d x$ (b) hearts at $t_{0}, t_{1}, t_{5}$ and $t_{10}$ of Nav1.5blocking. a. Symmetry breaking evident at $\mathbf{t}_{10}$ with an APD so $_{\text {gradient }} \sim \mathbf{3 5 m s}$. APs consistently selected from 4 sites for all measurements on the map longitudinally (white dots, numbers: 1,3; corresponding blue colored APs on the left) and transversely (red dots, numbers: 2,4; red APs on the left) perfectly overlap at $\mathrm{t}_{0}$. APD 50 dispersion maps are shown on the right of the APs. At $t_{0}$, the map shows a homogeneous distribution of APD values in the tissue, with prolongation at the pacing site (P). APD 50 is defined as the duration between the times at which the green dotted line crosses the AP (the $10 \%$ lower threshold) to the $50 \%$ repolarization from the peak (pink dotted line). APD prolongation is noticeable as Flecainide progresses (the gray dotted line within the APs at $t_{1}, t_{5}$ and $t_{10}$ represents the initial AP at $\mathrm{t}_{0}$ ). The map shows a steady APD prolongation manifested by the appearance of the lighter blue color. A striking $\mathrm{APD}_{50}$ distribution appears at $\mathrm{t}_{10}$, where the $\mathrm{LV}$ epicardium embeds simultaneously an area with prolonged APs (red) abutting another area with paradoxically shortened APs (blue). This bistability is also reflected in the selected APs that clearly separate into 2 groups (prolonged and shortened), independent of direction of propagation. b. APD prolongation in the $\boldsymbol{m d x}$ is not associated with a symmetry breaking process at $\mathbf{t}_{10}$. The $m d x$ APs prolong with Flecainide. Homogeneously, the dispersion maps manifest this prolongation without diverging from the circular distribution. Consequently, all APs remain superimposable. Annotations in figure: $\mathbf{A o}=$ Aorta, $\mathbf{A p}=$ Apex, $\mathbf{R}=$ Right, $\mathbf{L}=$ Left, $\mathbf{P}=$ Pacing, $\mathbf{P E}=$ Pacing electrode. Scale bar $\mathbf{m a p}=1 \mathrm{~mm}$. Scale $\mathbf{b a r} \mathbf{A P}=20 \mathrm{~ms}$. 
In Figure 28, APs and $\mathrm{APD}_{50}$ dispersion maps for WT (a) and $m d x$ (b) at $\mathrm{t}_{0}, \mathrm{t}_{1}, \mathrm{t}_{5}$ and $\mathrm{t}_{10}$ of Flecainide $[1 \mu \mathrm{M}]$ are shown. The same mask of the LV free wall has been repeatedly used for the same heart preparation to reproduce the maps at different measurements as the drug treatment progresses. The APs were selected from 4 different locations on the map, chosen along the longitudinal direction of propagation (white dots on map, numbered as 1 and 3, corresponding blue colored APs on the left) and the transversal direction (red dots on map, numbered as 2 and 4, corresponding red colored APs on the left). The AP morphological changes were monitored at $t_{1}, t_{5}$ and $t_{10}$ as Nav1.5 blocking progressed lxvii. For a direct comparison, an underlying control AP is marked in a dotted gray line. The corresponding APD dispersion maps appear at the right side of the AP at all times. At $\mathrm{t}_{0}$ (Figure 28a, upper right), APs perfectly overlap and the dispersion map shows a homogeneous distribution of $\mathrm{APD}_{50}$, with higher values at the stimulation site, where current-induced electrotonic imbalance possibly causes a local increase in APD values. APD for the mouse heart is maximal at the pacing site and dispels in all directions as the wave moves away elliptically from the pacing site to the boundaries (the last sites of activation in a 2D projection). This circular distribution of APD values appears in all dispersion maps where breaking of symmetry did not kick in. Henceforth, AP sites were chosen along the longitudinal and transversal directions as to avoid the high electrotonic imbalance due to pacing at the center of the ventricle and the effects due to the boundaries (in the 2D map). Dispersion maps where the sinus rhythm $(8.3 \mathrm{~Hz}$ ) prevailed (not shown) were devoid from such a ramification. Therefore, the heart curvature (the heart being an ellipsoid-like object) was eliminated as a potential cause for the spatial APD distribution in this context. In addition to visualizing the gradient in APD values, histograms can be extracted from these maps (Figure 29) in order to quantify APD dispersion ( $\triangle$ APD) from the difference between the $95^{\text {th }}$ and $5^{\text {th }}$ percentile of the APD distribution 560 . For instance, from the WT histogram at $t_{0}$ (Figure $29 \mathrm{~b}$, left), $\triangle \mathrm{APD}_{50 \text {,WT }}$ was calculated as $95 \%-5 \%$ of the distribution $=36.7-24.5=12.2 \mathrm{~ms}$, with a median $=6.2 \mathrm{~ms}$.

At 1 min post-treatment initiation, the overlapping APs already show a prolongation starting from phase 1, a higher plateau phase then down to complete repolarization. The map shows a steady prolongation of $\mathrm{APD}_{50}$ manifested by the appearance of the lighter blue color. Further stretching of the APD occurs at $t_{5}$, with the map showing continuous elongation of the depicted values at each pixel. The histograms of the map at $t_{5}$ (not shown) form a Gaussian distribution with a median at $44.8 \mathrm{~ms}$ and $\triangle \mathrm{APD}_{50, \mathrm{WT}}=30.7 \mathrm{~ms}$. These values confirm that the APD in all pixels did indeed prolong, with a shift of the median of $\sim 15 \mathrm{~ms}$ to the right and a widening of the unimodal distribution base by $\sim 150 \%$ compared to the value at $\mathrm{t}_{0}$. A striking $\mathrm{APD}_{50}$ pattern appears at $\mathrm{t}_{10}$, where the $\mathrm{LV}$ free wall epicardium is observed to simultaneously accommodate two juxtaposed zones as a response to the same steady-state entrainment frequency $(10 \mathrm{~Hz})$. This symmetry breaking effect is characterized by two adjacent zones: One with prolonged APDs (red) and another with paradoxically shortened APDs (blue) separated by a borderline of middle APD values. Although histograms are not sensitive to the spatial distribution of APDs, particularly when the heterogeneity is global and involves large areas of the $\mathrm{LV}$, they revealed that the Gaussian distribution previously observed at $\mathrm{t}_{5}$ had separated into a bimodal one with a wider base (Figure $29 \mathrm{~b}$, left). At $\mathrm{t}_{10}$, the calculated median $=38.7 \mathrm{~ms}$ and $\triangle \mathrm{APD}_{50, \mathrm{WT}}=35.8 \mathrm{~ms}\left(\sim 193 \%\right.$ wider than $\triangle \mathrm{APD}_{50, \mathrm{WT}}$ at $\left.\mathrm{t}_{0}\right)$. The $\mathrm{APD}_{50}$ map at $\mathrm{t}_{10}$ for the sinus rhythm (dropped to $5 \mathrm{~Hz}$ after $10 \mathrm{~min}$ of Flecainide) reveals longer and shorter APDs with the same pattern as the paced rhythm $(10 \mathrm{~Hz})$. Hence this effect is pacing-independent and this functional APD heterogeneity is characteristic of the substrate. Along with a shallower upstroke (dotted gray line), this bistability was also apparent in the selected APs (Figure 28a, lower right) that clearly separated into two groups (prolonged and shortened) towards the end of phase 1, prior to the plateau phase independent of the direction of propagation. The APs with the contracted plateau approach the non-

Ixvii Refer to the Experimental Methods section 2.2.3, where calculation of the APD and the creation of APD dispersion maps are fully described. 
treated AP dotted line at the time of 50\% repolarization despite an initially shallower upstroke and a widened notch, to break away afterwards with a protracted repolarization until resting potential is reached, resulting in a marked abbreviation of the epicardial response at $50 \%$ repolarization.
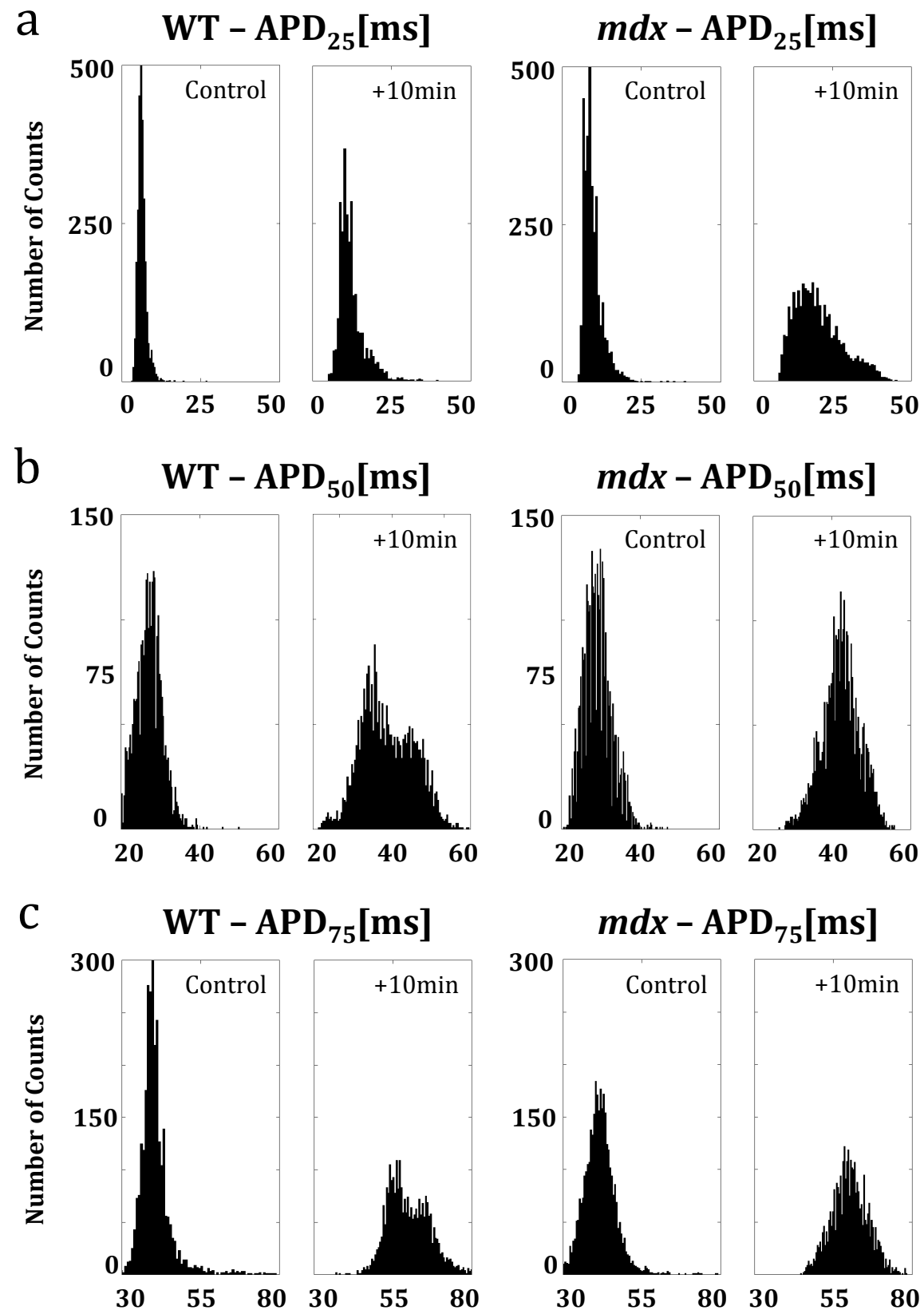

Figure 29. Histograms extracted from APD maps at different repolarization rates for $m d x$ and $W T$, at $t_{0}$ and $t_{10}$ of Nav1.5 blocking. a. APD 25 peaks at $6.2 \mathrm{~ms}$ and $8.9 \mathrm{~ms}$ at $\mathrm{t}_{0}$ for WT and $m d x$ respectively. $\triangle \mathrm{APD}_{25, m d x}$ approximates to $10.2 \mathrm{~ms}$ at to and increases to $30.9 \mathrm{~ms}$ at $\mathrm{t}_{10}$. This dispersion is considerably larger than the one calculated for the WT (evident from the widening of the distribution base), where $\triangle \mathrm{APD}_{25, \mathrm{WT}}=14.6 \mathrm{~ms}$ at $\mathrm{t}_{10}$. b. APD $_{50}$ distributions at $t_{0}$ do not differ between $\mathrm{WT}$ and $m d x$. Indeed their respective median values (WT $=30.5 \mathrm{~ms}, \quad m d x=$ $31.7 \mathrm{~ms}$ ) and $\triangle \mathrm{APD}_{50}$ (WT $=$ $12.2 \mathrm{~ms}, \quad m d x=13.1 \mathrm{~ms}$ ) are comparable. The bistability profile in the WT heart induced by Flecainide at $t_{10}$ is reflected by a bimodal distribution of values with a significant expansion of the dispersion parameters $\left(\triangle \mathrm{APD}_{50, \mathrm{WT}}=34.8 \mathrm{~ms}\right.$ $\left.\Delta \mathrm{APD}_{50, m d x}=25.6 \mathrm{~ms}\right)$. The distribution in the $m d x$ remains one-peaked, due to the absence of symmetry breaking as in the WT. c. APD 75 histograms show almost the same response as $A P D_{50}$, however the dispersion at $t_{0}$ of the $m d x$ values is larger $\left(\triangle \mathrm{APD}_{75, m d x}=20.4 \mathrm{~ms}\right.$ vs. $\left.\triangle \mathrm{APD}_{75, \mathrm{WT}}=13.5 \mathrm{~ms}\right) . \mathrm{A}$ bimodal pattern appears in the WT at $\mathrm{t}_{10}$, similar to the one of $\mathrm{APD}_{50}$ with $\triangle \mathrm{APD}_{75, \mathrm{WT}}=33.1 \mathrm{~ms}$. No such configuration appears in the $m d x$ heart at $\mathrm{t}_{10}$, where $\Delta \mathrm{APD}_{75, m d x}(=24.8 \mathrm{~ms})$ is smaller as well.

The changes in the $m d x$ heart with Flecainide are less complex (Figure 28b). At $t_{0}$ in this example, qualitatively the map is homogeneous with prolonged APDs mainly at the site of pacing. This is also evident from the Gaussian distribution of $\mathrm{APD}_{50}$ values in Figure 29b (right). The histograms are centered at $31.7 \mathrm{~ms}$, and have a $13.1 \mathrm{~ms}$ dispersion, a slightly higher value that the one of the WT heart at the same conditions $\left(\triangle \mathrm{APD}_{50, \mathrm{wT}}=12.2 \mathrm{~ms}\right)$. As for the $m d x$ APs (Figure 28b, left), they are characterized by a more flattened "notch" of the early repolarization phase (compared to WT APs at $\mathrm{t}_{0}$ ), preceding the higher plateau, until repolarization kicks-in to bring the APs to an almost similar 
termination as the WT APs. The bar graphs in Figure 30 - (a) $\mathrm{APD}_{50}$ along the longitudinal direction, (b) $\mathrm{APD}_{50}$ along the transversal direction - show that no significant difference exists between the $\mathrm{APD}_{50}$ values of WT $(\mathrm{N}=20)$ and $m d x(\mathrm{~N}=14)$ at $\mathrm{t}_{0}$, with $\mathrm{APD}_{50, \mathrm{WT}}=27.2 \pm 5.2 \mathrm{~ms}$ and $\mathrm{APD}_{50, m d x}=$ $28.1 \pm 5.6 \mathrm{~ms}$ longitudinally; $\mathrm{APD}_{50, \mathrm{WT}}=30.1 \pm 6.4 \mathrm{~ms}$ and $\mathrm{APD}_{50, m d x}=29.5 \pm 3.8 \mathrm{~ms}$ transversally.

Figure 30. Bar graphs show no significant difference in the progression of $A D_{50}$ between WT and $m d x$. a. Longitudinally, APD50 values in both groups show a prolongation with Flecainide. The initial comparability (at $\mathrm{t}_{0}$ ) between WT and $m d x$ is maintained in all following measurements. $\mathrm{APD}_{50, \mathrm{WT}}$ appears to prolong on average more than $\mathrm{APD}_{50, m d x}$ at $\mathrm{t}_{1}$ and $\mathrm{t}_{3}$, but the latter become slightly more prolonged on average afterwards. The increase in the SD at later stages accounts for the symmetry-breaking effect seen in the WT hearts (SD at $t_{0}=5.2 \mathrm{~ms}$ vs. SD at $\mathrm{t}_{10}=10.5 \mathrm{~ms}$ ). The percentile increase in $\mathrm{APD}_{50}$ between $\mathrm{t}_{10}$ and $\mathrm{t}_{0}$ is $\sim 65 \%$ and $\sim 70 \%$ for the WT and $m d x$ respectively. b. Transversally, a transient nonsymmetrical surge in APD 50,w $_{\text {, occurs only }}$ in the early few minutes of treatment. Initially, the WT and $m d x$ don't show any difference in the $\mathrm{APD}_{50}$ values. The significant difference between the two groups appears at $t_{1}$ and $t_{3}$ due to the inability of the APD $D_{50, m d x}$ to cope with the APD ${ }_{50, w T}$ prolongation pace. When comparing the longitudinal and transversal APD $50, \mathrm{WT}$, they are analogous at these two time points, which means that there are no directional preferences in the APD prolongation in the WT (see text). This is followed by an offset at $t_{5}$, where the two groups seem to reach a similar steady prolonged state (from $t_{5}$ to $t_{10}$ ). The $S D$ is however larger in the WT group at $\mathrm{t}_{10}$ $\left(\mathrm{APD}_{50, \mathrm{WT}}=48.1 \pm 9.4 \mathrm{~ms}\right.$ vs. $\mathrm{APD}_{50, m d x}=$ $49.9 \pm 4.8 \mathrm{~ms}$ ). The percentile increase in $\mathrm{APD}_{50}$ between $t_{10}$ and $t_{0}$ is $\sim 60 \%$ and $\sim 69 \%$ for the WT and $m d x$ respectively. Annotations in figure: * $(p<0.05)$; n.s. not significant.

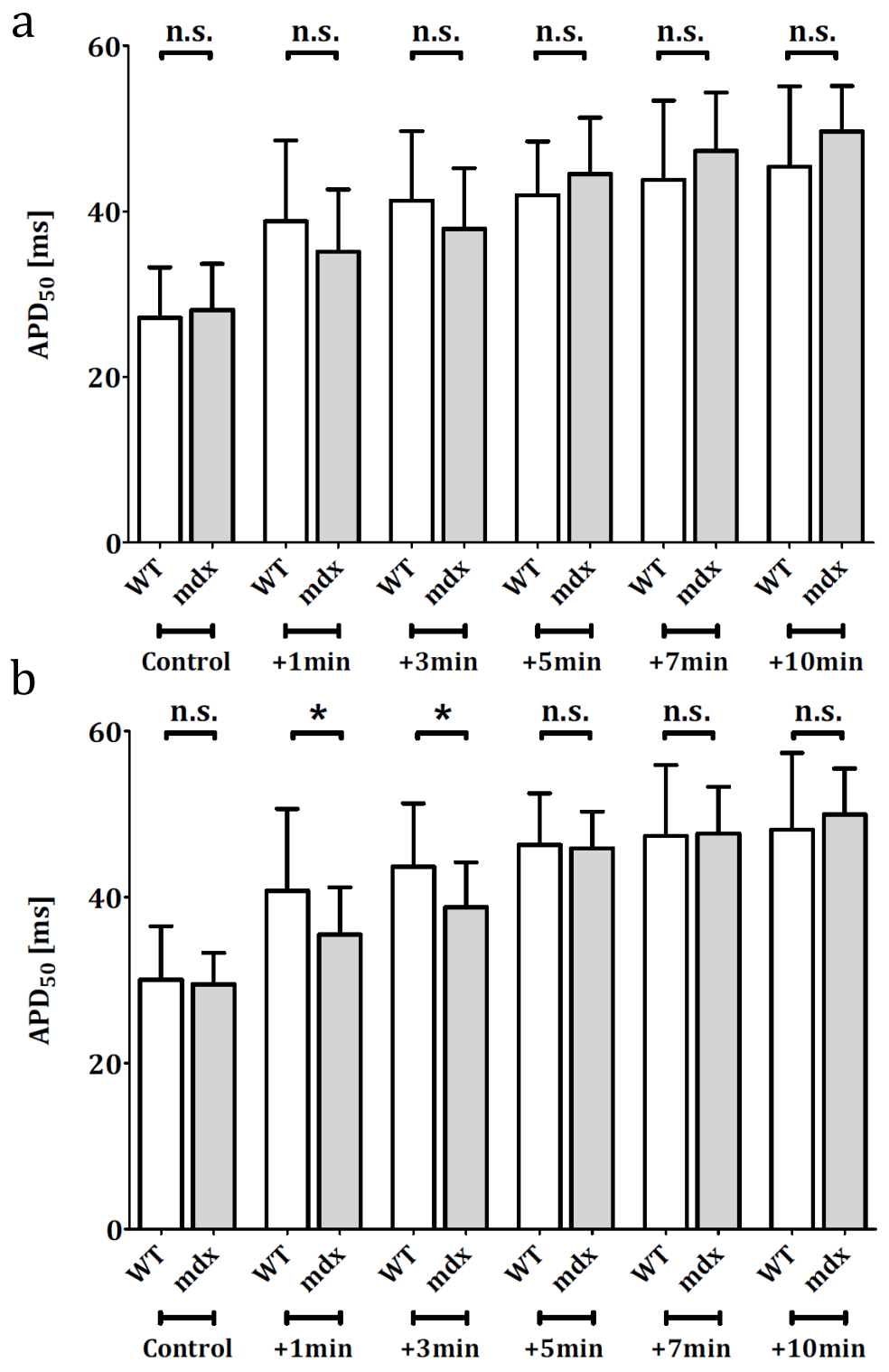

Consistently with the WT heart, the dystrophic heart also shows an overall prolongation of the $\mathrm{APD}_{50}$ at $\mathrm{t}_{1}$. In this example, the prolongation starts at a more delayed phase than observed in the WT and involves primarily the parts of the AP that follow the plateau. The dark blue color is concentrated towards the apex and the base of the heart (Figure 29b, upper right). Monitoring these areas over time revealed that prolongation of the APs occurred at a lower rate than the remaining of the map. At this time point of the experiment, $\mathrm{APD}_{50, m d x}$ acquired different statistics depending on the direction of propagation compared to its WT counterpart. Although at $t_{1}$ for example, APD50,WT (Long.) $=38.8 \pm 9.8 \mathrm{~ms}$ and $\mathrm{APD}_{50, \mathrm{WT}}$ (Trans.) $=40.8 \pm 9.8 \mathrm{~ms}$ are analogous on average, evident of no directional bias in the prolongation process of the APD, the difference between the WT and $m d x$ groups became statistically significant, possibly because of the smaller SD in the $m d x$ group along 
the transversal direction, at these consecutive time points ( $t_{1}$ and $\left.t_{3}\right)$. The APs at $t_{5}$ present with a distinctive form (compared to the $\mathrm{AP}_{m d x}$ at control conditions, marked by the dotted gray line with the APs at $t_{5}$ ), where the shallower upstroke merges with a dissolved notch followed by a higher plateau and extended repolarization. Importantly, Nav1.5 blocking appears to affect all APs symmetrically, in such a way that the map maintains its circular distribution of $\mathrm{APD}_{50}$ and the APs (on the left) remain superimposable. At $t_{10}$, the map and APs configurations show no evidence of a bistability or a symmetry breaking pattern despite continuous prolongation of the APs. This observation was consistent throughout all $m d x$ experiments $(\mathrm{n}=7)$. As an illustration, the histograms extracted from the $\mathrm{APD}_{50, m d x}$ map at $\mathrm{t}_{10}$ return a symmetrical distribution of $\mathrm{APD}_{50}$ values, centered at $46.1 \mathrm{~ms}$ with $\triangle \mathrm{APD}_{50, m d x}=25.6 \mathrm{~ms}\left(\sim 95 \%\right.$ increase in the width of $\triangle \mathrm{APD}_{50, m d x}$ compared to $\left.\mathrm{t}_{0}\right)$. Ostensibly, the dispersion of the $\mathrm{APD}_{50, m d x}$ is considerably smaller than the one recorded for the WT dual peaks distribution with $\triangle \mathrm{APD}_{50, \mathrm{wT}}=35.8 \mathrm{~ms}$ at $\mathrm{t}_{10}$ (which accounts for an actual increase of $\sim 190 \%$ in the WT case).

The APs presented in Figure 29a indicate that the splitting at $t_{10}$ is not a global effect on the timescale of an AP. It's rather confined to defined phases: All epicardial WT APs, whether abbreviated or prolonged, share similar shallow upstrokes and a uniform increased delay between phase 0 and 2 . The differential responsiveness to Flecainide commenced at the beginning of phase 2, where the dome (plateau) was almost completely lost in the APs that retracted. One may therefore question the degree at which $\mathrm{Ca}^{2+}$ could possibly be involved in this mechanism, tipping a network of epicardial cells to either acquire a prolonged or a protracted APD. Preceding the plateau phase, all APs share the same morphological properties. $\mathrm{APD}_{25}$ maps and histograms were therefore necessary to confirm the spatial distribution of the values and their corresponding statistics. The shortened APs however don't perfectly overlap with the control AP at later stages either (beyond $50 \%$ repolarization, Figure 29a, lower right). Therefore, whether the APs decided to keep a dome or lose it with Flecainide, the later stages of repolarization remained prolonged compared to control conditions. This being said, AP responsiveness at $25 \%$ and $75 \%$ repolarization is shown in Figure 31 (a. WT and b. $m d x$ ). In panel (a), $\mathrm{APD}_{25}$ (upper, right) dispersion map at $\mathrm{t}_{10}$ displays a homogeneous pattern with the highest APD at the pacing site, a similar configuration to the control conditions $\left(\mathrm{t}_{0}\right)$, however with more extended $\mathrm{APD}_{25}$ values in all directions. Histograms of $\mathrm{t}_{0}$ and $\mathrm{t}_{10} \mathrm{APD}_{25}$ maps are shown in Figure 29a (left). The narrow high-peak histograms at $t_{0}$ are centered at $6.1 \mathrm{~ms}$ with a dispersion that doesn't exceed $6.2 \mathrm{~ms}$. With Flecainide, this distribution widens at $\mathrm{t}_{10}$ to incorporate higher $\mathrm{APD}_{25}$ values resulting in a median of $10.2 \mathrm{~ms}$, with a dispersion of $14.6 \mathrm{~ms}(\sim 135 \%$ wider compared to $t_{0}$ ). In contrast, $\mathrm{APD}_{75}$ map at $\mathrm{t}_{10}$ (Figure $31 \mathrm{a}$, lower right) imitates the bimodal pattern observed for $\mathrm{APD}_{50}$, possibly with a smaller gradient between the high and low values. The histograms follow the map with a two-peaks pattern and a more extended dispersion (at $t_{0}$, median $=39.7 \mathrm{~ms}, \Delta \mathrm{APD}_{75, \mathrm{WT}}=13.5 \mathrm{~ms}$; at $\mathrm{t}_{10}$, median $=55 \mathrm{~ms}, \Delta \mathrm{APD}_{75, \mathrm{WT}}=33.1 \mathrm{~ms}$, i.e. an overall increase of $\sim 140 \%$ in $\left.\triangle \mathrm{APD}_{75, \mathrm{WT}}\right)$.

The $m d x$ maps in Figure $31 \mathrm{~b}$ show a homogeneous distribution of $\mathrm{APD}_{25}$ and $\mathrm{APD}_{75}$ at control conditions. The circular pattern of APDs persisted in both maps after $10 \mathrm{~min}$ of exposing the hearts to Flecainide, with no emergence of heterogeneity patterns at either level of repolarization. In this example, the values displayed in the $\mathrm{APD}_{25, m d x}$ map are considerably larger than the ones of the WT's $\left(t_{10}\right)$ and more diverse. It's expected that the $m d x$ histograms contain higher $\mathrm{APD}_{25}$ values (median at $\mathrm{t}_{10}=18.7 \mathrm{~ms}$ vs. WT median at $\mathrm{t}_{10}=10.2 \mathrm{~ms}$ ) with a wider dispersion (at $\mathrm{t}_{10}: \Delta \mathrm{APD}_{25, \mathrm{mdx}}=25.9 \mathrm{~ms}$ vs. $\triangle \mathrm{APD}_{25, \mathrm{WT}}=14.6 \mathrm{~ms}$ ). Between the experimental groups, $\mathrm{APD}_{25}$ remained significantly higher in the $m d x$ group over all time point measurements (Figure 32a, where longitudinal $\mathrm{APD}_{25}$ values are displayed in bar graphs. The transversal values reported the same statistical findings). It's obvious that the SD considerably increases in both groups (WT, $\mathrm{N}=20$ and $m d x, \mathrm{~N}=14$ ) as the treatment proceeds. For instance at $t_{0}, \mathrm{APD}_{25, \mathrm{WT}}=6.0 \pm 0.8 \mathrm{~ms}$ and $\mathrm{APD}_{25, \mathrm{mdx}}=9.5 \pm 1.9 \mathrm{~ms}$ ( $p$-value $\left.<0.0001\right)$. These 
values increase to $17.6 \pm 8.5 \mathrm{~ms}$ and $24.4 \pm 8.6 \mathrm{~ms}$ respectively at $t_{10}(p$-value $<0.05)$. The effect doesn't reflect a heterogeneity component with each heart (as for the SD of the $A P D_{50, w T}$ at $t_{10}$ ), since no symmetry breaking occurs neither in the $m d x$, nor in the WT at this level of repolarization. Instead it accounts for the widely spread values within each of the WT and $m d x$ groups over all experiments.

Figure 31. APs and APD 25/75 dispersion maps for WT (a) and $\operatorname{mdx}(\mathrm{b})$ hearts at $\mathrm{t}_{0}$ and t10 of Nav1.5-blocking. a. Symmetry breaking evident at $t_{10}$ only in the APD 75 map with a gradient $\sim 33 \mathrm{~ms}$. APs on the left of each map represent the 4 sites on the map from which they were evaluated (See caption of Figure 28). At $t_{10}$, symmetry breaking occurs before the commencement of the AP plateau. Hence the $\mathrm{APD}_{25, \mathrm{wT}}$ is devoid of the bimodal pattern, which is depicted instead in the $\mathrm{APD}_{75, \mathrm{WT}}$ dispersion map. $\triangle \mathrm{APD}_{75, \mathrm{WT}}\left(\right.$ at $\left.\mathrm{t}_{10}\right)=33.1 \mathrm{~ms}$, a value $\sim 20 \mathrm{~ms}$ larger than the one at $t_{0}$. b. APD prolongation and widening of the dispersion values in the $m d x$ heart is devoid of any heterogeneity breakup in the medium. (Upper, right) Prolongation of $\mathrm{APD}_{25}$ values in the $m d x$ heart is evident at $\mathrm{t}_{10}$, associated with $\Delta \mathrm{APD}_{25, m d x}=25.9 \mathrm{~ms}$. The dispersion in APD75,mdx values at $t_{10}$ is slightly higher (24.8ms) than the calculated one at $\mathrm{t}_{0}(20.4 \mathrm{~ms})$. The pattern remains circular and unremarkable (lower, right). Annotations in figure: Ao = Aorta, $\mathbf{A p}=$ Apex, $\mathbf{R}=$ Right, $\mathbf{L}=$ Left, $\mathbf{P}=$ Pacing, $\mathbf{P E}=$ Pacing electrode. Scale bar map = $1 \mathrm{~mm}$. Scale bar AP $=20 \mathrm{~ms}$.

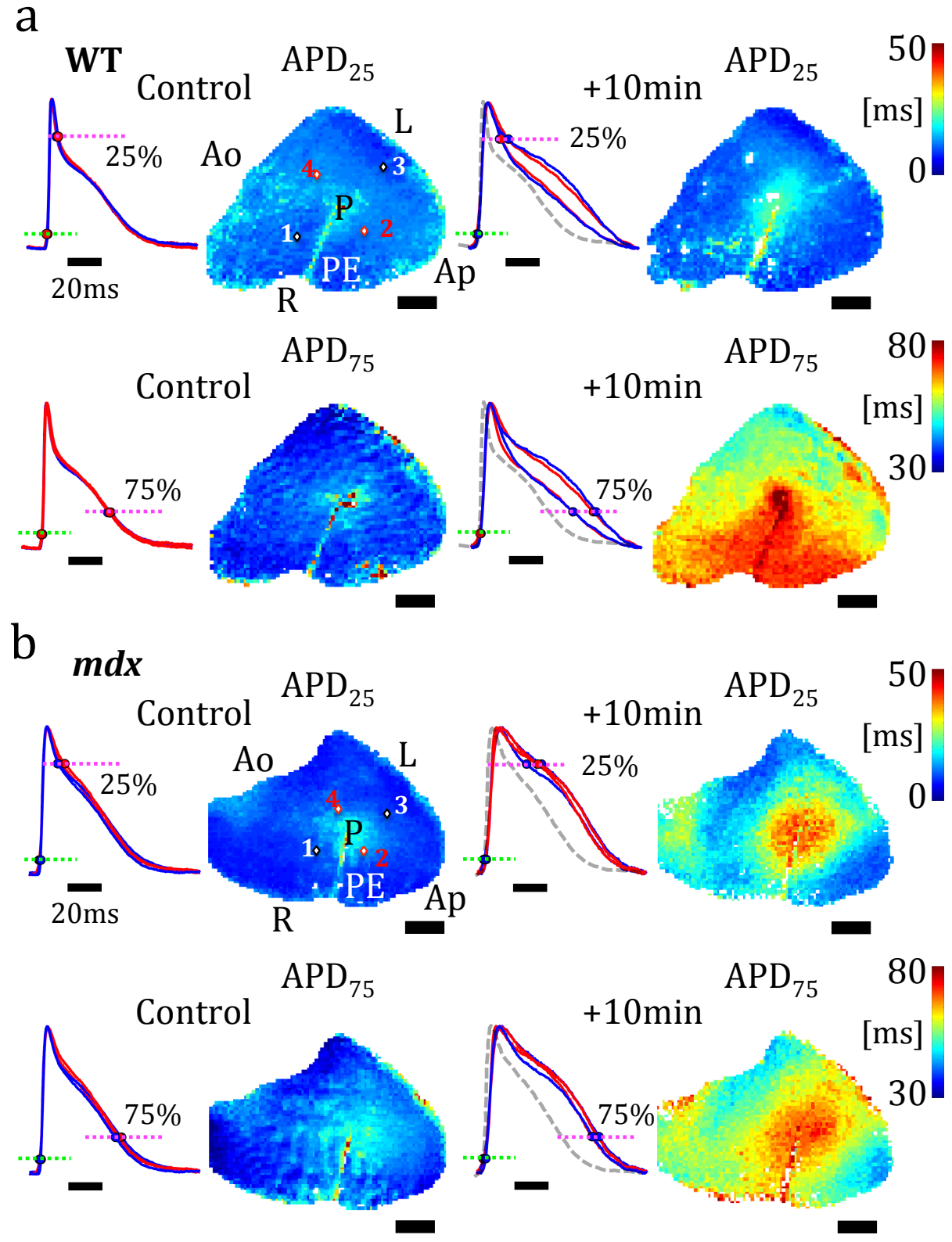

Measuring $\mathrm{APD}_{25}$ in the context of $\mathrm{Na}_{\mathrm{V}} 1.5$ blocking appears "labile" regardless of the investigated substrate (WT or $m d x$ ). On one hand, some hearts responded acutely to Flecainide, once it was introduced in the medium. Prolongation was perceived starting $t_{1}$ or $t_{3}$ and was maintained across the next following measurements. On the other hand, some hearts showed a more conservative prolongation of their APD at 25\% repolarization, compared to the effect of Flecainide observed at $50 \%$ or $75 \%$ repolarization levels. These hearts maintained lower $\mathrm{APD}_{25}$ values throughout the entire experimental protocol. Pooling the data gathered over all experiments revealed a sparse distribution of $\mathrm{APD}_{25}$ values, responsible for the increasing $\mathrm{SD}$ at each time point with Flecainide. 


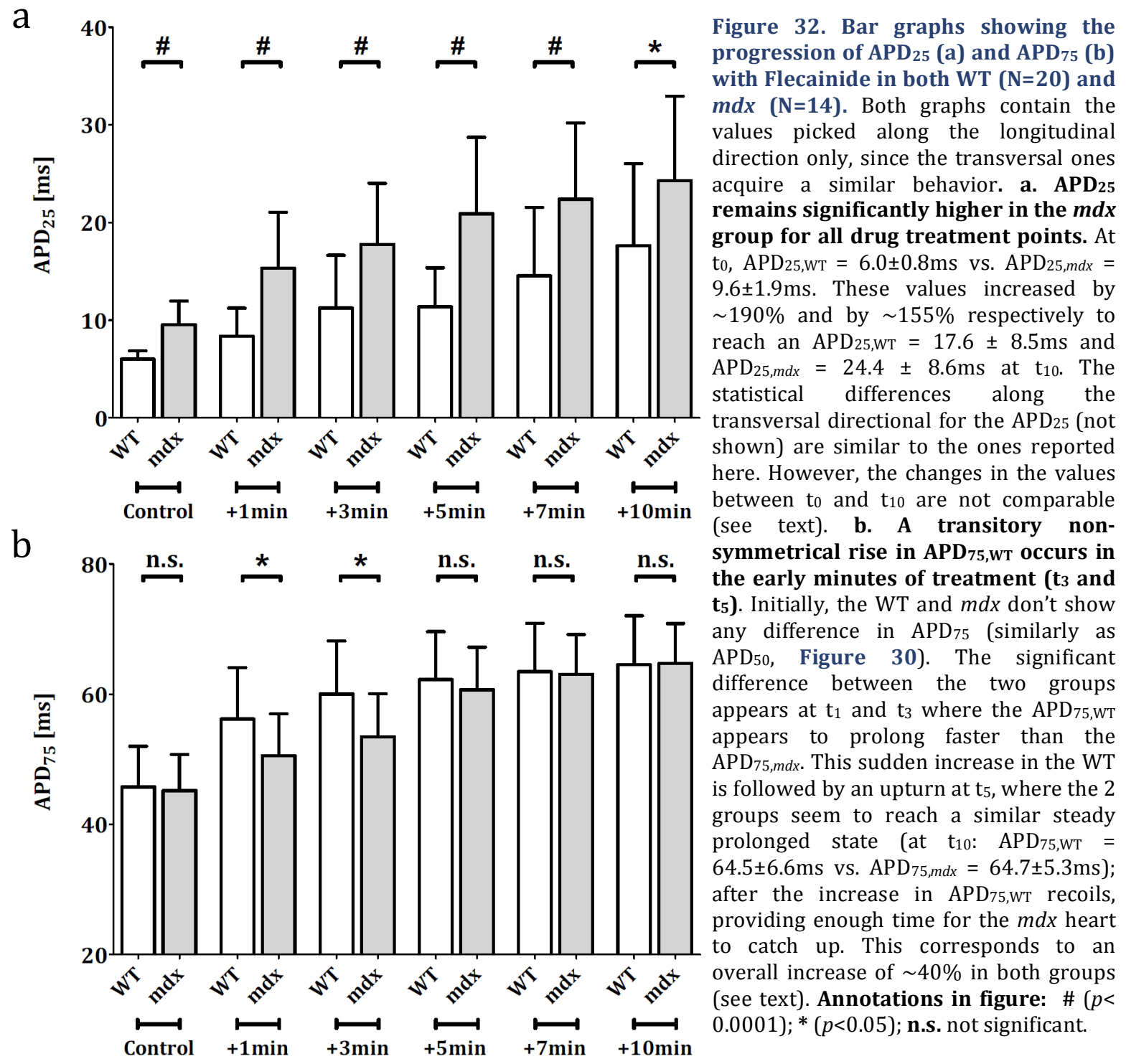

It's obvious that all APDs (at all repolarization levels, here in terms of duration, not dispersion) were affected by Flecainide. The variability of APDs are a response to the drug was however not identical. Both $\mathrm{APD}_{50}$ and $\mathrm{APD}_{75}$ increased with the drug and did not show any directional bias in either experimental group (i.e. the increase in APD in one direction is comparable to the increase in the other direction). In the WT, $\mathrm{APD}_{50, \text { Long }}$ prolongs by $\sim 65 \%$ and $\mathrm{APD}_{50, \text { Trans }} \sim 60 \%$. Almost similar values were picked up for the $m d x$ with an increase in $\mathrm{APD}_{50 \text {, Long }} \sim 70 \%$ and $\mathrm{APD}_{50, \text { Trans }} \sim 69 \%$ between $t_{0}$ and $t_{10}$. APD 75 in WT and $m d x$ prolonged by $\sim 40 \%$ in both directions. Furthermore, $\mathrm{APD}_{90}$ showed an analogous increase in the WT and $m d x$ of $\sim 30 \%$ in either direction. It can be inferred that not only the later stages of repolarization are less affected than the early ones, but also APD prolongation secondary to Flecainide use becomes more direction- and substrate- independent as the AP approaches full repolarization. $\mathrm{APD}_{25}$ exhibited the strongest susceptibility to Nav1.5blocking, with remarkable differences in direction within the same group and among the groups. The WT longitudinal $\mathrm{APD}_{25}$ scores the highest relative change between $t_{0}$ and $t_{10}$ with an increase that exceeds $190 \%$. The transversal $\mathrm{APD}_{25}$ (WT) increases by $\sim 170 \%$. These effects are significantly 
reduced in the $m d x$ heart with $\sim 155 \%$ and $110 \%$ prolongation in $\mathrm{APD}_{25}$ along the longitudinal and transversal directions respectively (Table in Figure 34). These dominant changes account for the continuous statistical difference between the two groups notably seen in $\mathrm{APD}_{25}$, at each and every time point of the treatment. Contrastingly, APD dispersion may not be quantitatively deduced directly from the APD changes acquired with Flecainide; even though the SD increase at $t_{10}$ for either group reflects a degree of inhomogeneity in the population. For instance, the mere observation that no statistically significant difference exists between WT and $m d x$ at $\mathrm{t}_{10}$ for $\mathrm{APD}_{50}$ (Figure 30 ) and $\mathrm{APD}_{75}$ (Figure $32 \mathrm{~b}$ ) does not eliminate the possibility that differences in dispersion maps are qualitatively and quantitatively more pronounced between the two groups at these repolarization levels (Figure 28, Figure 29). Indeed, the symmetry breaking effect arising with Flecainide at $t_{10}$ in both $\mathrm{APD}_{50}$ and $\mathrm{APD}_{75}$ WT maps was reflected with a widening of the maps' respective histograms by $\sim 190 \%\left(\triangle \mathrm{APD}_{50, \mathrm{WT}}\right)$ and $\sim 140 \%\left(\triangle \mathrm{APD}_{75, \mathrm{WT}}\right)$, whereas the dispersion in the $m d x$ maps didn't exceed $90 \%$ and $20 \%$ for $\Delta \mathrm{APD}_{50, m d x}$ and $\Delta \mathrm{APD}_{75, m d x}$ respectively. These numbers cannot have been concluded from APD values alone.

Figure 33. Effects of Flecainide on APD dispersion for WT and $m d x$ at $25 \%, 50 \%$ and $75 \%$ AP repolarization. $\mathrm{APD}_{\mathrm{xx}}$ is calculated for each prep at $t_{0}$ and $t_{10}$ as the difference between the $95^{\text {th }}$ and $5^{\text {th }}$ percentile of $\mathrm{APD}_{\mathrm{xx}}$ distribution. It shows a significant difference in dispersion for $\mathrm{APD}_{50}$ and $\mathrm{APD}_{75}$ at $\mathrm{t}_{10}$ as a result of symmetry breaking that widens the APD distribution in their respective maps. The disperison of $\mathrm{APD}_{25, m d x}$, unlike later repolarization levels, is significantly larger than the WT's at $t_{0}$. This difference dissipates with Nav1.5 blocking despite a widening of $\sim 200 \%$ and $\sim 120 \%$ of $\triangle \mathrm{APD}_{25}$ for the WT and $m d x$ respectively. Annotations in figure: ${ }^{* *}(p$-value $<0.01){ }^{*}(p$ value $<0.05)$; n.s. not significant.

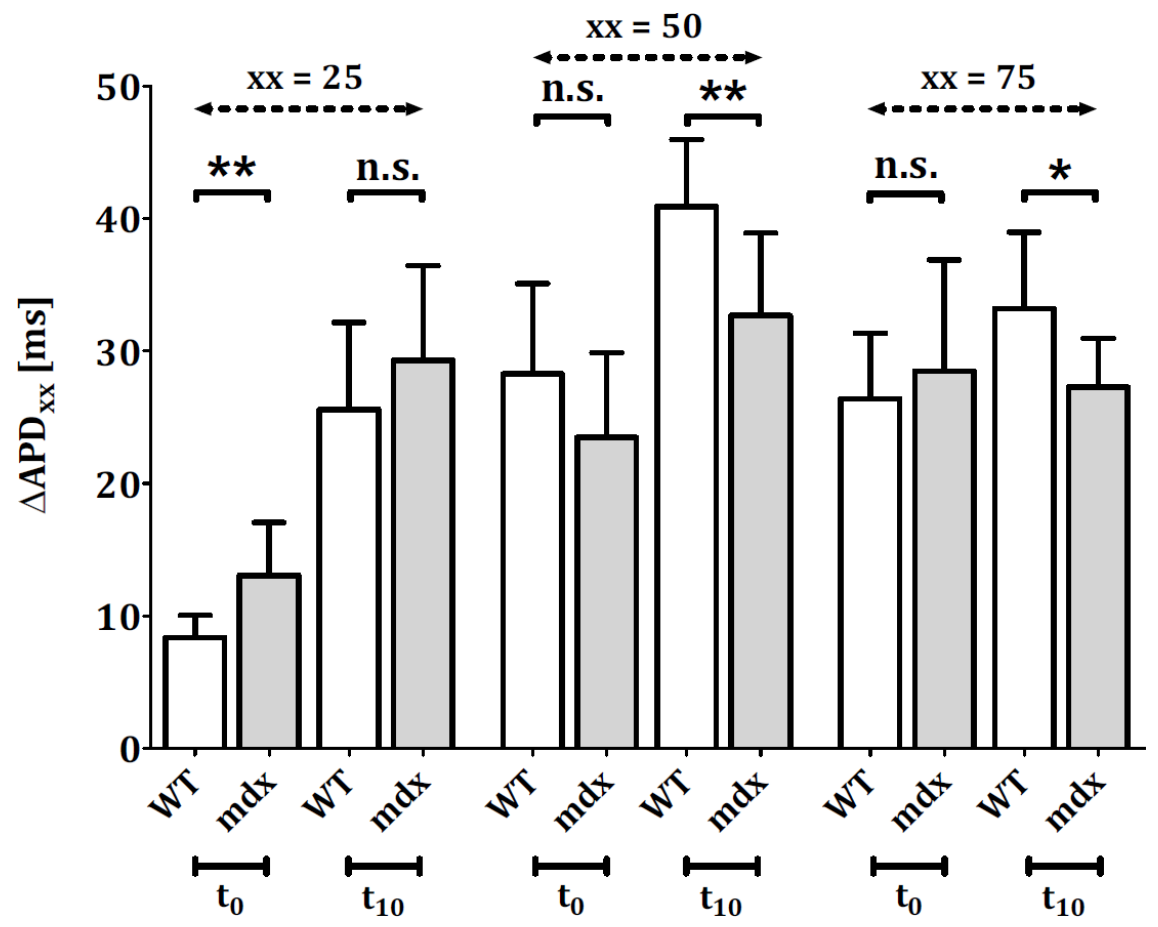

We suspect from the previous examples that the discrepancy detected in the expansion of $\mathrm{APD}_{\mathrm{xx}}$ and $\triangle \mathrm{APD}_{\mathrm{xx}}\left(\mathrm{xx}\right.$ : repolarization level, $25 \%, 50 \%$ or $75 \%$ ) with $\mathrm{Na}_{\mathrm{V}} 1.5$ blocking could be present in the other data sets, which necessitated analysing dispersion in all maps as a separate entity from the APDs. Figure 33 contains bar graphs where APD dispersion is compared to the WT $(n=10)$ and dystrophic substrates $(\mathrm{n}=7)$ at different repolarization levels under control and drug exposure conditions. At $\mathrm{t}_{0}$, only $\triangle \mathrm{APD}_{25}$ is significantly larger in the $m d x$ group, with $\triangle \mathrm{APD}_{25, \mathrm{WT}}=8.4 \pm 1.7 \mathrm{~ms}$ and $\Delta \mathrm{APD}_{25, m d x}=13.1 \pm 4.0 \mathrm{~ms}$ ( $p$-value $\left.<0.01\right)$. Interestingly, the dispersion of $\mathrm{APD}_{50}$ maps is on average larger than the one calculated for the $m d x$, though the difference between the two groups isn't significant $\left(\triangle \mathrm{APD}_{50, \mathrm{WT}}=28.3 \pm 6.8 \mathrm{~ms} ; \quad \Delta \mathrm{APD}_{50, m d x}=24.5 \pm 6.4 \mathrm{~ms} ; \quad p\right.$-value $\left.=0.15\right)$. At $75 \%$ repolarization, both WT and $m d x$ return almost identical values for their respective APD dispersion with $\triangle \mathrm{APD}_{75, \mathrm{WT}}=26.4 \pm 4.9 \mathrm{~ms}$ and $\triangle \mathrm{APD}_{75, m d x}=32.2 \pm 5.8 \mathrm{~ms}$. All $\triangle \mathrm{APD}_{\mathrm{xx}}$ increase at $\mathrm{t}_{10}$ Flecainide, except for $\triangle \mathrm{APD}_{75, m d x}$ which remains almost unchanged at $\mathrm{t}_{10}\left(\triangle \mathrm{APD}_{75, m d x}=27.3 \pm 3.7 \mathrm{~ms}\right)$. The increase in $\triangle \mathrm{APD}_{75, \mathrm{WT}}(33.2 \pm 5.8 \mathrm{~ms})$ with almost no concomittant change in the $m d x$ counterpart gave rise to the statitistically significant difference between the two groups at $t_{10}(p$-value $<0.05)$. The change in 
$\triangle \mathrm{APD}_{25}$ is by far the most remarkable with an increase that exceeds $200 \%$ in the WT and $120 \%$ in the $m d x$ (at $\mathrm{t}_{10}: \Delta \mathrm{APD}_{25, \mathrm{WT}}=25.6 \pm 6.6 \mathrm{~ms} ; \Delta \mathrm{APD}_{25, m d x}=29.3 \pm 7.1 \mathrm{~ms}, p$-value $=0.29$ ). Ideally, the decrease in upstroke velocity and AP amplitude secondary to Nav1.5 blocking anticipiates a degree of encrochement on $\mathrm{APD}_{25}$, due to the temporal and voltage dependence of the succeding currrents $\left(\mathrm{I}_{\mathrm{to}, 1}\right.$ and $\left.\mathrm{I}_{\mathrm{Ca}}\right)$ on upstroke kinetics. In other words, if Flecainide were an ideal Nav1.5-blocker, it will exert its influence more prominently on the early phases of the AP, rather than the later ones. This correlation is further delineated in Figure 35, where concurrent changes in AP parameters $\left(A P D_{25}\right.$ vs. $\left.(\mathrm{dF} / \mathrm{dt})_{\max }\right)$ are plotted during the progressive development of Nav1.5 blocking. While $\mathrm{APD}_{25}$ remains significantly more prolonged in the $m d x$ with Flecainide compared to the WT at all times (Figure 32a), dispersion doesn't seem to exactly adopt this behavior. This can be partly explained by the fact that dispersion additionally has a spatial component that surpasses the local effects of the upstroke, in consequence cannot be precisely predicted by simply monitoring changes in the upstroke, as it might be the case for $\mathrm{APD}_{25}$ in physiological conditions.

\begin{tabular}{|c|c|c|c|c|}
\hline \multirow{3}{*}{$\xi$} & & $\% \mathrm{APD}_{25}$ & $\% \Delta \mathrm{APD}_{25}$ & \multirow{5}{*}{$\begin{array}{l}\text { Figure } 34 \text {. Table summarizing the percentage increase in } \\
\text { APD }_{25} \text { between to and } t_{10} \text { with Flecainide, along with the } \\
\text { corresponding increase in dispersion. The } m d x \text { heart with } \\
\text { lower Nav1.5 shows a more restrained increase in its APD } \\
\text { parameters compared to its WT counterpart. More importantly, } \\
\text { the increase in dispersion in the WT heart exceeds the } \\
\text { prolongation of the APD in either direction, whereas in the } m d x \text {, it } \\
\text { doesn't }\end{array}$} \\
\hline & Long. & 190 & \multirow[t]{2}{*}{200} & \\
\hline & Trans. & 170 & & \\
\hline \multirow{2}{*}{$\frac{\mathfrak{x}}{\mathfrak{s}}$} & Long. & 155 & \multirow[t]{2}{*}{120} & \\
\hline & Trans. & 110 & & \\
\hline
\end{tabular}

The two-states system that erupts in the WT heart at $\mathrm{t}_{10}$ manifests itself by the significant increase in $\triangle \mathrm{APD}_{50, \mathrm{WT}}(40.9 \pm 5.0 \mathrm{~ms})$ and $\triangle \mathrm{APD}_{75, \mathrm{WT}}(33.2 \pm 5.8 \mathrm{~ms})$ that outruns any change happening in the $m d x$ heart (Figure 33), which is devoid of symmetry breaking $\left(\Delta \mathrm{APD}_{50, m d x}=32.7 \pm 6.2 \mathrm{~ms}, \Delta \mathrm{APD}_{75, m d x}\right.$ $=27.3 \pm 3.7 \mathrm{~ms}$ ). The widening of histograms' base in the corresponding WT APD $\mathrm{D}_{50}$ and $\mathrm{APD}_{75}$ maps is a result of rather than a trigger for this bistable state. The assumption behind this lies in the observation that the acquired high spatial gradient between the two adjacent zones of the WT heart after 10 min exposure to Flecainide has only become more obvious at later phases of the AP (i.e. at $50 \%$ and $75 \%$ repolarization). At these levels, Flecainide, being an open state channel blocker, has actually little -if any- effect on Nav1.5 channels ${ }^{561},{ }^{562}$. We believe that if the mechanistic trigger for this bistability is a $\mathrm{Na}_{v} 1.5$-dependent phenomenon (i.e. an aftermath of Nav1.5 inhibition), it shall lie most probably somewhere in the earlier phases of the AP, within a time interval limited to the maximum upstroke velocity on one side and the genesis of plateau phase on the other side. This time interval is wrapped within $\mathrm{APD}_{25}$.

To elucidate how the different phases of the epicardial AP could influence one another during the gradual development of $\mathrm{Na}_{\mathrm{v}} 1.5$ blocking, we examined the correlation between the decrease in maximum upstroke velocities $(\mathrm{dF} / \mathrm{dt})_{\max }$ and the concomitant changes in $\mathrm{APD}_{25}$ as Flecainide proceeded. The values in Figure 35 were obtained from APs at 4 different locations on the LV epicardium ( 2 along the longitudinal axis of propagation and 2 others along the transversal, as previously described) for WT $(\mathrm{N}=20)$ and $m d x(\mathrm{~N}=14)$. Figure $35 \mathrm{a}$ shows that under control conditions, the relationship between $\mathrm{APD}_{25}$ and $(\mathrm{dF} / \mathrm{dt})_{\max }$ is not linear. The clustering of the points is well delineated within each group, indicating a specific influence of the upstroke velocities on determining $\mathrm{APD}_{25}$, although this influence isn't quantitatively explicit. However, for fast enough upstrokes, the times scales of the processes occurring afterwards are so small that $\mathrm{APD}_{25}$ is brief. It's also tempting to speculate that for faster upstroke velocities $\mathrm{APD}_{25}$ will become tangential to a minimum, beyond which no further dependence on the upstroke can be assessed. Since the number of resting Nav1.5 channels in a cell is finite, the stochastic nature of ionic channels determines 
opening probability of these channels once threshold is reached. This returns a finite value for the fast inward $I_{\mathrm{Na}}$ and upstroke velocity measured at the level of the cell. Although the increase in membrane voltage $\left(\mathrm{V}_{\mathrm{mbr}}\right)$ activates L-type $\mathrm{Ca}^{2+}$ channels during the upstroke ${ }^{563}$, $\mathrm{I}_{\mathrm{Ca}, \mathrm{L}}$ does not contribute substantially to the rising phase of the AP (partly due to much slower kinetics of the $\mathrm{Ca}^{2+}$ channels compared to $\left.\mathrm{Na}_{\mathrm{V}} 1.5\right)^{74}$. $\mathrm{I}_{\mathrm{to}, 1}$ with its outward rectification properties has voltage- and timedependencies on the preceding upstroke amplitude and velocity. The time scales of these processes are also finite and can be modulated by the same factors that modify the upstroke's properties. In a virtual cell, where all $\mathrm{Na}_{v} 1.5$ channels open simultaneously (when $\mathrm{V}_{\mathrm{mbr}}>\mathrm{V}_{\mathrm{thr}}$ ), $A P \mathrm{D}_{25}$ would still acquire a finite value not lower than the time scales needed for $I_{t o, 1}$ and $I_{C a}$ activation (middle processes in action). Following this line of thought, the blue squares appear as if forming a line parallel to the $\mathrm{x}$-axis, where further increments in $(\mathrm{dF} / \mathrm{dt})_{\max }$ only shorten $\mathrm{APD}_{25}$ by a value limited to measurement error $(0.5 \mathrm{~ms})$.

Figure 35. Plots illustrating the correlation between concurrent changes in $\mathrm{APD}_{25}$ and $(\mathrm{dF} / \mathrm{dt})_{\max }$ during the gradual development of Nav1.5 blocking. a. At to, the points within each group cluster in a well-demarcated fashion. Points with high $(\mathrm{dF} / \mathrm{dt})_{\max }$ correspond to shorter $A P D_{25}$. As the upstroke velocity decreases, $\mathrm{APD}_{25}$ progressively increases. b. At $t_{5}$, all points shift to the left and upward, indicating a continuously increasing $\mathrm{APD}_{25}$ with lower $(\mathrm{dF} / \mathrm{dt})_{\max }$. The capacity of $(\mathrm{dF} / \mathrm{dt})_{\max }$ to influence $\mathrm{APD}_{25}$ values is still consistent and not completely lost until $5 \mathrm{~min}$ later (c) where the intermingled data points in different patterns illustrate a pronounced decoupling effect between $(\mathrm{dF} / \mathrm{dt})_{\max }$ and $\mathrm{APD}_{25}$.

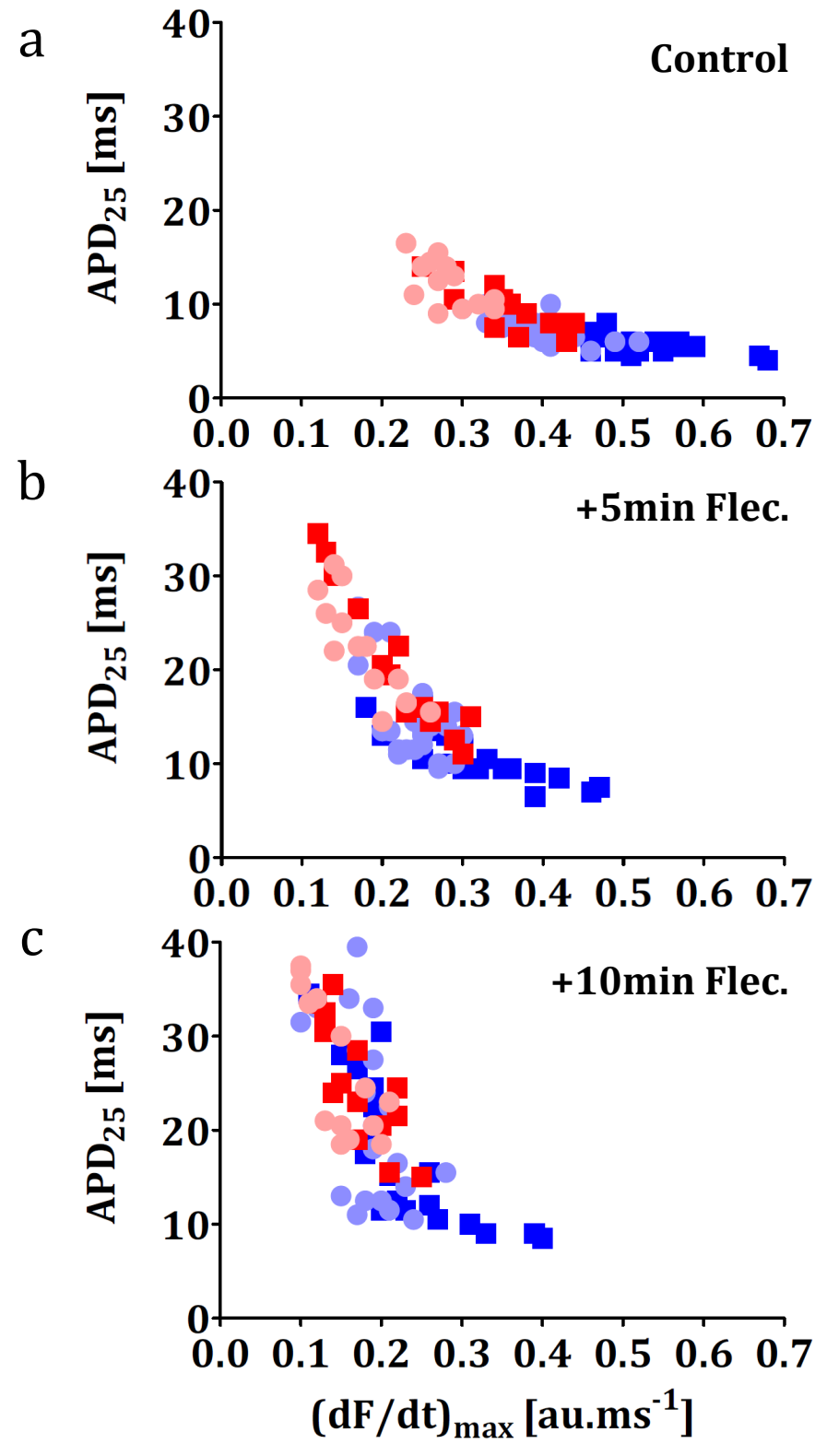

Long. Locations - WT

Long. Locations - $m d x$ Trans. Locations - WT
Trans. Locations - $m d x$ 
As the values of $(\mathrm{dF} / \mathrm{dt})_{\max }$ are lower along the transversal direction, $\mathrm{APD}_{25}$ increases slightly with a maximal deviation of $3 \mathrm{~ms}$ compared to the ones measured in the direction perpendicular to it (mean values at $\mathrm{t}_{0}: \mathrm{APD}_{25, \mathrm{wT}}$ (Long) $=6.0 \pm 0.8 \mathrm{~ms}$ and $\mathrm{APD}_{25, \mathrm{wT}}$ (Trans) $=7.5 \pm 1.5 \mathrm{~ms}$ ). Loss of Nav1.5 in the $m d x$ leads to a global slowing of the upstroke velocities that seemingly associate with larger $\mathrm{APD}_{25}$ values. In Figure 35a, red/pink symbols occupy the left side and upward. The thin blue tail relates well with the fact that $\mathrm{SD}$ in $\mathrm{APD}_{25, \mathrm{wT}}$ is smaller than the one observed in the $m d x$ in either direction (mean values at $t_{0}: \mathrm{APD}_{25, m d x}$ (Long) $=9.6 \pm 1.9 \mathrm{~ms}$ and $\mathrm{APD}_{25, m d x}$ (Trans) $=12.2 \pm 2.4 \mathrm{~ms}$ ). The latter translates into a wider distribution of the red/pink symbols on the left side of the plot. With Flecainide exposure, all points move further to the left and spread over a wider area (Figure 35b). This is consistent with a decrease in $(\mathrm{dF} / \mathrm{dt})_{\max }$ in all preps and corresponds to a global prolongation in $\mathrm{APD}_{25}$ associated with an increase in SD within each group. As $(\mathrm{dF} / \mathrm{dt})_{\max }$ becomes weaker, its capacity to regulate $\mathrm{APD}_{25}$ is also impaired and the time scales of the middle processes become progressively more important. At $t_{10}$, as the rising part of AP takes considerably more time to reach its peak, $(\mathrm{dF} / \mathrm{dt})_{\max }$ modulation of $\mathrm{APD}_{25}$ is almost lacking and the middle processes take over (Figure 35c). As the points from all groups intermingle in disorganized patterns, a direct coupling between $\mathrm{APD}_{25}$ and $(\mathrm{dF} / \mathrm{dt})_{\max }$ becomes ambiguous and doubtful. As illustrated previously in Figure 26 , Flecainide gradually diminishes the extent to which the upstroke velocity could modulate $\mathrm{CV}$ until $\mathrm{t}_{10}$, where local heterogeneity in the medium breaks out decoupling $(\mathrm{dF} / \mathrm{dt})_{\max }$ and CV almost entirely.

\subsection{Expatiated Pathophysiological Heterogeneity in $\triangle K P Q$}

Among the different pathologies classified under LQTS is a subgroup of genetic defects that are linked to mutations in $\mathrm{Na}_{v} 1.5^{392}$ (LQTS3). One of the most severe defects so far studied is a deletion mutation of nine bases that code for Lysine-1505, Proline-1506 and Glutamine-1507 in the intracellular linker between DIII and DIV of Nav1.5 $\alpha$-subunit (SCN5a- $\Delta \mathrm{KPQ}$ ) $393,395,396$. One of the widely accepted arrhythmia-facilitating mechanisms in LQTS3 is failure of Nav1.5 to inactivate properly, hence it's a gain-of-function mutation. Could AADs that target $\mathrm{I}_{\mathrm{Na}, \mathrm{f}}$ have a potential therapeutic effect through indirect modulation of $\mathrm{I}_{\mathrm{Na}, \mathrm{p}}$, possibly decreasing the propensity for arrhythmia in LQTS3? Foremost, the existing Nav1.5 blockers remain up-to-date non-ideal and their proarrhythmic side effects have overshadowed their therapeutic potentials in many circumstances ${ }^{36,474}$. Therefore it would be legitimate to assume that the outcome of pharmacological interventions with mutated channels would be fairly unpredictable.

Interactions of $\mathrm{Na}_{\mathrm{v}} 1.5$ with pharmacological molecules could be dramatically altered, whether mutations involve parts of the channel proximal ${ }^{349}$ or distant from the receptor site ${ }^{564}$. The everincreasing complexity of the relationship between $\mathrm{Na}_{v} 1.5$ genetic mutations and clinical phenotypes muddles the ability to predict whether defective channels could behave similarly to the wild type ones in the presence of blocking agents, inevitably when mutations alter the time a channel resides in a particular conformational state ${ }^{385}$. In this regard, Nav1.5 blockade with Flecainide in the context of mutated channels remains highly controversial $420,565,566$. Some studies reveal that Flecainide has possible therapeutic prospects in the management of arrhythmia in LQTS3 patients, since it was shown to normalize the QT-interval on ECG ${ }^{567-569}$. In other cases, Flecainide was reported to induce Brugada-like signs (ST segment elevation) in LQTS3 patients, henceforth increasing their risk for $\mathrm{SCD}^{570}$. In Brugada Syndrome (BS), the use of Flecainide is recommended as a diagnostic tool to unmask asymptomatic probands, since the drug evokes ST-elevation on ECG with high sensitivity and specificity 416,566 .

Recent experimental findings have revealed preferential blockade by Flecainide of $\mathrm{I}_{\mathrm{Na}, \mathrm{p}}$ in the $\Delta \mathrm{KPQ}$ model 565 . It was found that Flecainide has a more potent interaction with the carboxylic terminal of 
the mutated channel than with the WT counterpart, leading to the correction of the disease phenotype ${ }^{398,566}$. The precise mechanism for this preferential block in the mutated channel remains unclear, hence a subject for speculations. The authors suggested a shift of Nav1.5 availability in the hyperpolarizing direction might have enhanced Flecainide block in this substrate ${ }^{566}$. On the other hand, Flecainide, being an open state blocker ${ }^{561}$ of the WT channel, can become trapped during repolarization, i.e. closure of the activation gate may trap the drug molecule within the channel by blocking the exit pathway, independently of inactivation properties ${ }^{454}$. Hyperpolarization has been shown to slow down recovery from block in case of trapping, consequently leading to an enhancement of Flecainide's blocking effects ${ }^{571}$. Therefore, the effect of the mutation on the channel's interaction with the drug molecule is yet to be clarified.

In the next set of experiments, $\triangle \mathrm{KPQ}$ murine hearts were subjected to the same treatment protocol that was followed for the WT and $m d x$ heart (section 3.2). Over a period of $5 \mathrm{~min}$, the mutated hearts $(n=4)$ and their WT counterparts $(n=4)$ were perfused with Flecainide $[1 \mu \mathrm{M}]$. The same experimental conditions used previously were reproduced in the treatment of $\triangle \mathrm{KPQ}$ hearts. The exposure to the drug was limited to $5 \mathrm{~min}$ only (half the exposure time in the previous sets of experiments), due to difficulty in entraining the mutated hearts into a $10 \mathrm{~Hz}$ steady state pacing beyond 5min. From the previous experiments, the increased sensitivity of the WT group to Flecainide compared to a substrate with lower availability of $\mathrm{Na}_{v} 1.5$ was a major finding. This sensitivity was translated into a symmetry breaking effect that lead to the coexistence of two adjacent stable zones entrained by the same frequency. As the outcomes of Flecainide exposure are yet to be clarified, activation, functional heterogeneity profile and drug proarrhythmia are investigated next, since a $\mathrm{Na}_{\mathrm{v}} 1.5$ blocker might turn to be completely ineffective or overly effective in interacting with the mutant channel.

\subsubsection{Atypical Activation Spread in $\triangle K P Q$ with Flecainide}

The $\triangle \mathrm{KPQ}$ mutation destabilizes inactivation, as previously explained. Wang et al. measured $\mathrm{I}_{\mathrm{Na}}$ from a whole cell voltage clamp using $\triangle \mathrm{KPQ}$ mutated cardiac cells and reported that the steady state activation curve was slightly $(\sim 6 \mathrm{mV})$ shifted towards depolarizing voltages compared to the WT 395 . In addition, the fractional amplitude of the fast component of the $\mathrm{I}_{\mathrm{Na}, \mathrm{f}}$ was actually greater in the $\triangle \mathrm{KPQ}$ cells than the WT. The recovery from inactivation kinetics was also considerably faster for the mutated cells: in the WT the largest fraction (77\%) of channels seem to have recovered from inactivation with a time constant of $\tau_{\mathrm{WT}}=6.7 \mathrm{~ms}$ vs. $59 \%$ in $\Delta \mathrm{KPQ}$ at $\tau_{\Delta \mathrm{KPQ}}=2.1 \mathrm{~ms}^{395}$. Based on these cellular results, and assuming that the resting membrane potential $\left(\mathrm{V}_{\mathrm{rmp}}\right)$ in both substrates is unaltered, we do not expect the maximum upstroke velocity $(\mathrm{dF} / \mathrm{dt})_{\max }$ to be modified in $\triangle \mathrm{KPQ}$. As we have seen in the previous WT and $m d x$ study, $(\mathrm{dF} / \mathrm{dt})_{\max }$ may represent an index of Nav1.5 availability in some conditions, however may not necessarily give an accurate prediction of the $\mathrm{CV}$ in the medium.

In Figure 36a, the optical APs (OAP) were measured from the LV free wall of the WT and $\triangle \mathrm{KPQ}$. Prior to normalization, the OAPs were compared for amplitude and $\mathrm{FC}_{\%}$ (as previously described). The $\mathrm{AP}_{\triangle \mathrm{KPQ}}$ is superimposed over the dotted WT representation (same as the $\mathrm{AP}_{\mathrm{WT}}$, left) for a direct comparison. On the right, the slightly shallower upstroke is followed by a hampered notch, which precedes a plateau phase at more positive voltages. Unlike the $\mathrm{AP}_{m d x}$ (Figure 19, left), where the early prolongation phases are compensated with strong enough repolarization currents leading to an almost normal termination of the $\mathrm{AP}$, the $\mathrm{AP}_{\triangle \mathrm{KPQ}}$ is characterized by a continuous noncompensated prolongation and a considerably delayed repolarization. In that regard, the $\mathrm{AP}_{\triangle \mathrm{KPQ}}$ appears comprehensively prolonged compared to the $\mathrm{AP}_{\mathrm{WT}}$ in all phases (Figure $36 \mathrm{a}$, right). 


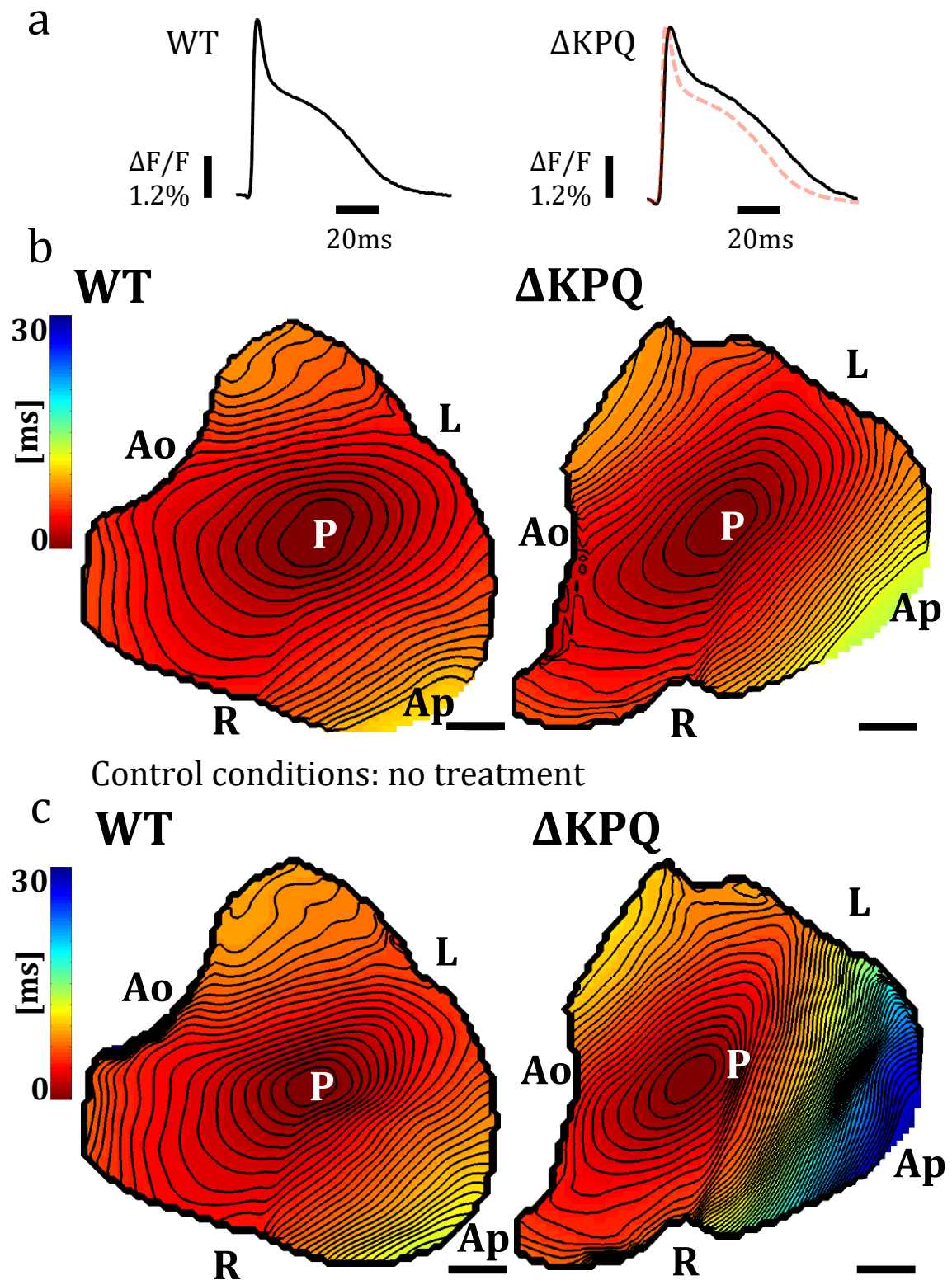

After 5 min treatment with Flecainide $(1 \mu \mathrm{M})$
Figure 36. Changes in AP and activation maps changes with Nav1.5 mutation and exposure to Flecainide $[1 \mu \mathrm{M}]$. a. Morphological changes due to AP prolongation detected optically in the $\triangle K P Q$ heart (BCL=100ms). As the $\mathrm{AP}_{\triangle \mathrm{KPQ}}$ is superimposed over the dotted $\mathrm{AP}_{\mathrm{WT}}$, the upstroke of the mutated AP appears shallower. The cascade of ionic events promotes a more flattened notch, a higher plateau phase and prolongation of the $\mathrm{AP}_{\triangle \mathrm{KPQ}}$ in all phases until repolarization is terminated. Scale bar $=20 \mathrm{~ms} . \Delta \mathbf{F} / \mathbf{F}=$ fractional change of the fluorescent signal. b. Activation maps at control conditions. From a point stimulation $\mathrm{P}$, the electrical wave anisotropically propagates across the LV free wall to the boundaries. Latency in propagation and compaction of isochronal lines in the $\triangle \mathrm{KPQ}$ map indicate a decrease in $\mathrm{CV}$ under control $\left(\mathrm{t}_{0}\right)$ conditions. c. Activation maps at $5 \mathrm{~min}\left(\mathrm{t}_{5}\right)$ of Flecainide exposure. While activation spread in the WT heart remains anisotropic, the mutated heart adopts an atypical propagation. As the position of the electrode remains unaltered, the wave emanating from the point stimulation shifts to the left and spreads quasi-elliptically. The wave fronts in the red area travel faster than the ones in the direction of the apex, where severe conduction slowing prevails. Annotations in figure: Scale $\quad$ bar $=1 \mathrm{~mm}, \quad$ Ao=Aorta, Ap=Apex, $\quad$ L=Left, $\quad \mathbf{R}=$ Right, $\mathbf{P}=$ Pacing.

Assigning activation times to all pixels gives rise to the activation maps in Figure $36 \mathrm{~b}$ and c. At control conditions $\left(\mathrm{t}_{0}\right)$, both WT and $\triangle \mathrm{KPQ}$ propagation patterns spread anisotropically from the tip of the pacing electrode to boundaries within almost the same allotted time, although isochrones along the transversal direction appear imperceptibly more compacted than in the WT map. Indeed from the activation maps in this example at $t_{0}$, conduction was evaluated along the longitudinal direction where $\mathrm{V}_{\max , \mathrm{WT}}=0.74 \pm 0.03 \mathrm{~m} . \mathrm{s}^{-1}$ vs. $\mathrm{V}_{\max , \Delta \mathrm{KPQ}}=0.69 \pm 0.08 \mathrm{~m} . \mathrm{s}^{-1}$ and along the transversal direction where $V_{\min , W T}=0.40 \pm 0.05 \mathrm{~m} . \mathrm{s}^{-1} \mathrm{vs}$. $\mathrm{V}_{\min , \Delta \mathrm{KPQ}}=0.27 \pm 0.04 \mathrm{~m} . \mathrm{s}^{-1}$. 
After $5 \mathrm{~min}$ exposure to Flecainide $\left(t_{5}\right)$, qualitative differences between the two maps reveal an atypical pattern of activation in the mutated heart. Meanwhile, the WT map maintains a global anisotropic propagation with slower components compared to $t_{0}\left(a t t_{5}: V_{\max , W T}=0.62 \pm 0.05 \mathrm{~m} \cdot \mathrm{s}^{-1}\right.$ and $V_{\min , W T}=0.21 \pm 0.03 \mathrm{~m}_{\mathrm{s}} \mathrm{s}^{-1}$ ). As the position of the pacing electrode remains unchanged (Figure 45), the wave clearly emanates from the tip of the electrode where the pacing current is introduced into the tissue. However it acquires a preferential shift towards the base where conduction is clearly faster (the first isochrone is located towards the aortic side), maintains an elliptical shape until the convex ends along the major axis of the ellipses reach the right and left boundaries of the heart (within the red colored area of the map), then resumes propagation towards the apex in quasi-planar waves. From the movie of the measurement (Figure 44 and Figure 45), an artifact due to electrode position change was ruled out. This pattern appears to be generic for all $\triangle \mathrm{KPQ}$ preps treated with Flecainide at $t_{5}(n=4)$ in this study. The cause of such activation is unknown. Hence, it's tempting to speculate that the mechanism behind the deviation from global anisotropy (to partial anisotropy) could possibly be due to a source-sink mismatch. A point stimulation, unlike a line stimulation, creates an elliptical pattern of activation founded on fiber orientation; principally due to "facilitation" of spread in the direction parallel to the fiber's long axis and increased "discontinuities" in the direction perpendicular to it 254 . In addition, planar waves have the propensity to travel faster than convex ones ${ }^{572}$, due to more efficient distribution of depolarizing charges as the wave front crosses the medium ${ }^{573}$. Since the depolarizing current was introduced into the epicardium from a point stimulation, it will give rise to ellipses with convex wave fronts. For unknown reasons, the upper half (from LV free wall center to base) of the heart appears to facilitate conduction and becomes fully activated within $15 \mathrm{~ms}$ of pulse induction $\left(\mathrm{V}_{\mathrm{max}, \triangle \mathrm{KPQ}}=0.44 \pm 0.04 \mathrm{~m} \cdot \mathrm{s}^{-1}\right)$. The remaining half (center to the apex) requires an additional $15 \mathrm{~ms}$ to fully activate $\left(\mathrm{V}_{\min , \Delta \mathrm{KPQ}}=0.11 \pm 0.02 \mathrm{~m} . \mathrm{s}^{-1}\right)$. In consequence, even if conduction block is not yet present here, double this time was still needed to reach the apex. As the elliptical activation terminates in the upper half of the heart, a quasi-planar wave front is formed, more capable of surpassing the electrical load across the remaining epicardium to the apex than the originally expected convex wave.

The difference in CV between the two groups as Flecainice proceeds is shown in Figure 37. Bar graphs for $\mathrm{V}_{\max }\left(\mathrm{N}=8\right.$, per group) and $\mathrm{V}_{\min }(\mathrm{N}=8$, per group) indicate a decrease in velocities in both substrates with an overall change (between $t_{0}$ and $t_{5}$ ) of $32 \%$ and $26 \%$ in $V_{\max }$ for WT and $\Delta \mathrm{KPQ}$ respectively; $40 \%$ and $39 \%$ in $V_{\min }$ for WT and $\triangle \mathrm{KPQ}$ respectively. It's noteworthy to emphasize that at this time point of the current protocol, the values reached in the WT group are consistent with what was reported in the previous section for the control group of the $m d x$ (during the 10min protocol), where at $t_{5}, V_{\text {max,WT }}$ and $V_{\text {min,WT }}$ decreased by $33 \%$ and $39 \%$ respectively (Figure 22). At control conditions, the velocities recorded for the $\triangle \mathrm{KPQ}$ substrate are globally $15-18 \%$ lower than the ones of the WT. The difference at that stage is significant between the two groups with $\mathrm{V}_{\text {max,WT }}=$ $0.78 \pm 0.10 \mathrm{~m} . \mathrm{s}^{-1}, \mathrm{~V}_{\mathrm{max}, \Delta \mathrm{KPQ}}=0.66 \pm 0.06 \mathrm{~m} . \mathrm{s}^{-1}$ ( $p$-value $\left.<0.05\right)$; and $\mathrm{V}_{\min , \mathrm{WT}}=0.37 \pm 0.08 \mathrm{~m} . \mathrm{s}^{-1}, \mathrm{~V}_{\min , \Delta \mathrm{KPQ}}=$ $0.31 \pm 0.03 \mathrm{~m} . \mathrm{s}^{-1}$ ( $p$-value<0.05). As Nav1.5 blocking proceeds, both velocities in the two substrates start to decrease, until $t_{5}$ where the difference in velocities between the groups vanishes. The percentage decrease $V_{\text {max }}$ is slightly higher for the WT group than it is for the $\triangle K P Q$, but $V_{\min }$ seems to be similarly affected in both groups at $t_{5}$. In the $10 \mathrm{~min}$ protocol used to compare $m d x$ and WT, patchy high velocity (or supernormal conduction) zones erupt at $t_{10}$ in the WT group along the longitudinal direction. In the current results, the protocol was stopped at $5 \mathrm{~min}$, without an area of supernormal conduction being detected in either group.

In Figure 38, $(\mathrm{dF} / \mathrm{dt})_{\max }$ is calculated at each pixel for each of the maps presented in Figure 36 . This approach is necessary to identify how Nav1.5 blocking alters $(\mathrm{dF} / \mathrm{dt})_{\max }$ in the $\Delta \mathrm{KPQ}$ substrate and whether the fluctuations detected in $\mathrm{CV}$ are reproducible for $(\mathrm{dF} / \mathrm{dt})_{\max }$. 


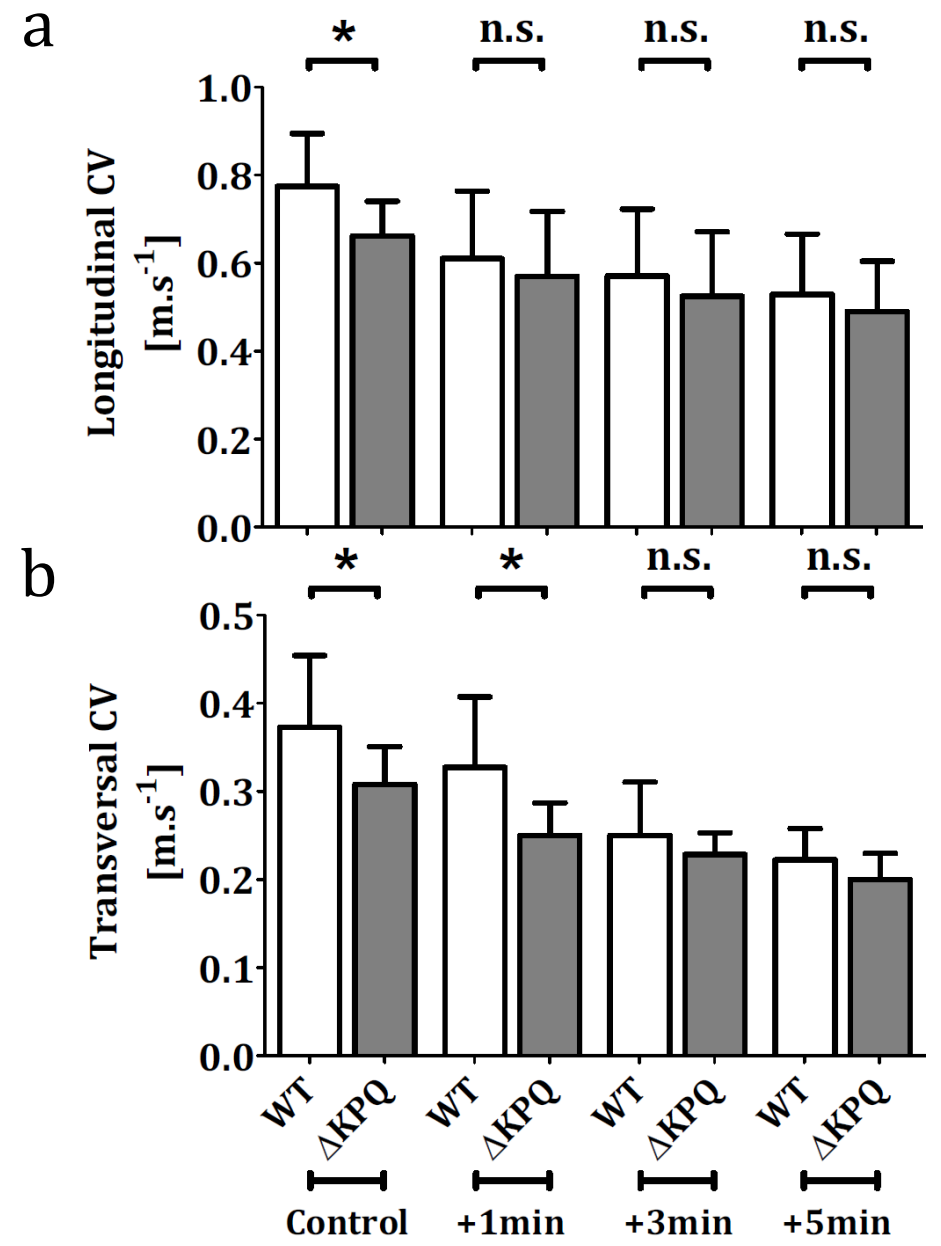

Figure 37. Bar graphs of $V_{\max }$ and $V_{\min }$ for WT $(\mathrm{N}=8)$ and $\triangle \mathrm{KPQ}(\mathrm{N}=8)$ at to and different time points of Flecainide $[1 \mu \mathrm{M}]$ perfusion. a. Decrease in $V_{\max }$ is consistent for both groups. At $t 5$, a maximum decrease of $\sim 32 \%$ was recorded in the WT group vs. $\sim 26 \%$ in the $\triangle \mathrm{KPQ}$ (To note: a similar value was recorded in the previous set of experiments in the $m d x$ control group $(\mathrm{N}=22)$ at $t_{5}$ with a decrease of $33 \%$, Figure 22). Despite a larger percentage decrease in the WT, both groups have almost identical velocities at $t_{5}\left(\mathrm{~V}_{\max , \mathrm{WT}}=0.53 \pm 0.12 \mathrm{~m} . \mathrm{s}^{-1}\right.$; $\left.\mathrm{V}_{\text {max }, \Delta \mathrm{KPQ}}=0.49 \pm 0.11 \mathrm{~m} . \mathrm{s}^{-1}\right)$. The initially larger velocity in the WT group at $t_{0}$ accounts for the larger decline $\left(\mathrm{V}_{\mathrm{max}, \mathrm{WT}}=0.78 \pm 0.10 \mathrm{~m} . \mathrm{s}^{-1}\right.$, $\mathrm{V}_{\text {max }, \Delta \mathrm{KPQ}}=0.66 \pm 0.06 \mathrm{~m} . \mathrm{s}^{-1}, p$-value $\left.<0.05\right)$. b. A gradual decrease in $V_{\min }$ with Flecainide. A total of $\sim 41 \%$ and $\sim 39 \%$ decrease in $V_{\text {min }}$ was recorded for $\mathrm{WT}$ and $\triangle \mathrm{KPQ}$ at $\mathrm{t}_{5}$ respectively. Starting with $\mathrm{V}_{\text {min, } W T}=0.37 \pm 0.08$ $\mathrm{m} . \mathrm{s}^{-1}$ and $\mathrm{V}_{\min , \Delta \mathrm{KPQ}}=0.31 \pm 0.03 \mathrm{~m} \cdot \mathrm{s}^{-1}(p-$ value $<0.05)$, the difference between the two groups becomes statistically insignificant at $5 \mathrm{~min}$ of Flecainide perfusion $\left(\mathrm{V}_{\text {min, } \mathrm{WT}}=\right.$ $0.22 \pm 0.03 \mathrm{~m} . \mathrm{s}^{-1}$ and $\left.\mathrm{V}_{\min , \Delta \mathrm{KPQ}}=0.19 \pm 0.03 \mathrm{~m} \cdot \mathrm{s}^{-1}\right)$. Annotations in figure: $*(p$-value<0.05), n.s. not significant.

In a similar fashion to $\mathrm{CV}, \mathrm{WT}$ and $\triangle \mathrm{KPQ}(\mathrm{dF} / \mathrm{dt})_{\max }$ dispersion maps show a decrease in all directions with Flecainide (Figure 38a). The maps' corresponding histograms are shown in Figure $40 \mathrm{c}$. In the leftmost panel, the WT upstroke velocities have a bimodal distribution with two peaks at $\sim 0.31$ and $\sim 0.44 \mathrm{~ms}^{-1}$, suggestive of the presence of longitudinal and transversal clusters. The $\Delta \mathrm{KPQ}$ map also shows a semi-bimodal configuration at control conditions, with the majority of values lingering in the lower range compared to WT peaking at $\sim 0.28 \mathrm{~ms}^{-1}$ and a tail of higher values in the vicinity of $0.36 \mathrm{~ms}^{-1}$. With Flecainide (at $t_{5}$ ), histograms shift to the left indicating lower range of values in the two substrates: the WT maintains the bimodal distribution with the highest frequency around $0.24 \mathrm{~ms}^{-1}$ and a smaller peak towards $0.35 \mathrm{~ms}^{-1}$; whereas the $\Delta \mathrm{KPQ}$ histograms concentrate to the left with a high peak around $0.1 \mathrm{~ms}^{-1}$ and another smaller one around $0.16 \mathrm{~ms}^{-1}$.

On average, the shallower upstrokes in the mutated substrate (upstroke $=0.43 \pm 0.03$ a.u.ms $\mathrm{m}^{-1}$ ) do not reveal a significant difference to their WT counterpart at $t_{0}\left(0.47 \pm 0.10\right.$ a.u.ms $\left.{ }^{-1}\right)$ with $p$-value $=$ 0.34 (Figure $38 \mathrm{~b}$ ). The sharper decrease in the $\triangle \mathrm{KPQ}$ heart with Flecainide alters this difference in favor of considerably larger values in the WT with $\sim 71 \%$ decrease in $\triangle \mathrm{KPQ}$ in the longitudinal direction (WT: $0.31 \pm 0.08$ a.u. $\mathrm{ms}^{-1} ; \Delta \mathrm{KPQ}: 0.15 \pm 0.04$ a.u. $\mathrm{ms}^{-1} ; p$-value $<0.05$ ), and $\sim 60 \%$ decrease in $\Delta \mathrm{KPQ}$ in the transversal direction (WT: $0.25 \pm 0.08$ a.u.ms ${ }^{-1} ; \Delta \mathrm{KPQ}: 0.14 \pm 0.04$ a.u. $\mathrm{ms}^{-1} ; p$-value $<0.05$ ). The decrease in the WT upstrokes, unlike the $\triangle \mathrm{KPQ}$ substrate, doesn't show directional differences, in such a way that the total change in $(\mathrm{dF} / \mathrm{dt})_{\max }$ is almost similar in the longitudinal $(\sim 36 \%)$ and transversal $(\sim 38 \%)$ directions. 


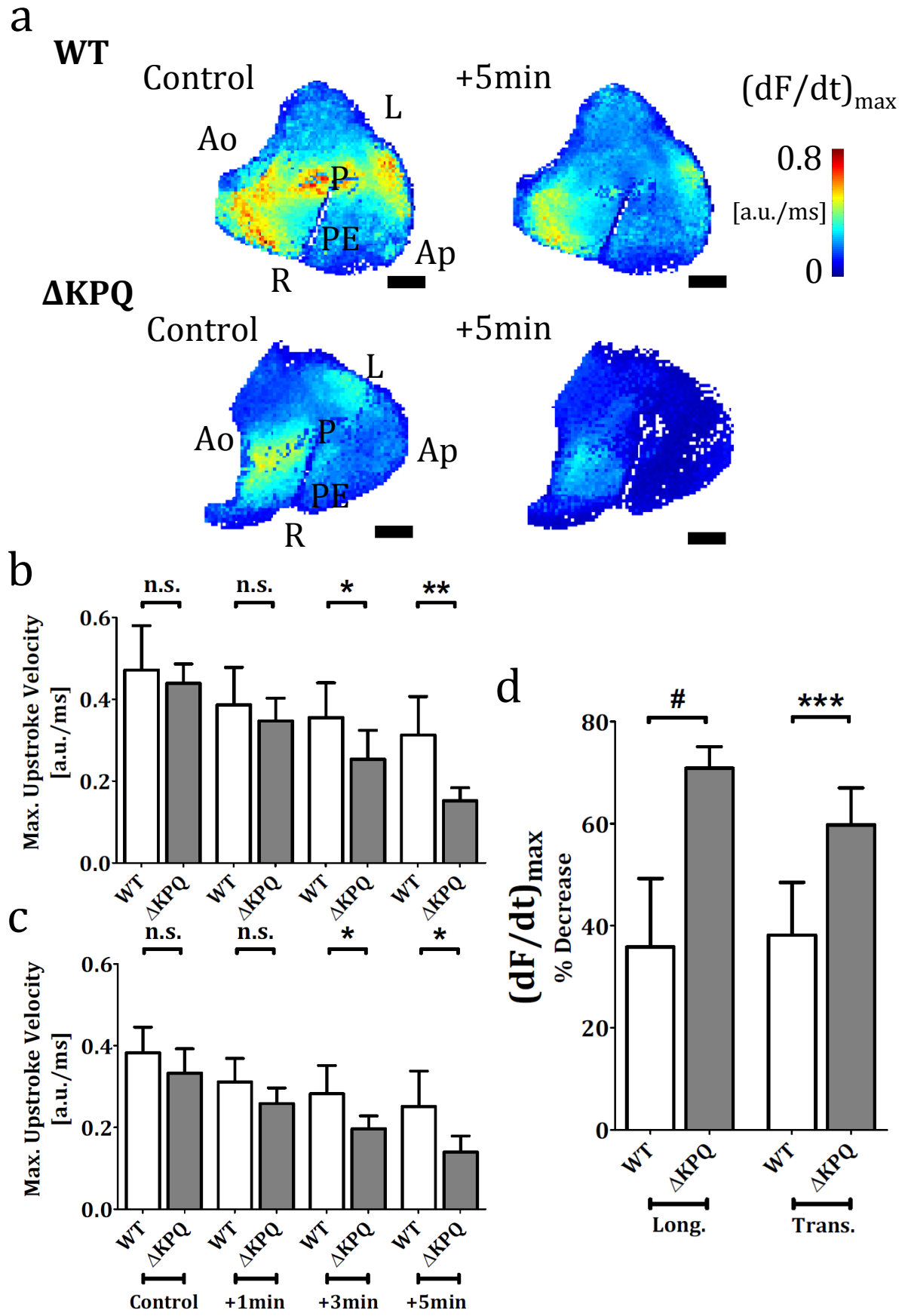

Figure 38. Dispersion maps showing at one pixel resolution the changes in $(\mathrm{dF} / \mathrm{dt})_{\max }$ with Flecainide $[1 \mu \mathrm{M}]$ in $\mathrm{WT}$ $(\mathrm{N}=8)$ and $\triangle \mathrm{KPQ}(\mathrm{N}=8)$ and their respective bar graphs. a. Anisotropic $(\mathrm{dF} / \mathrm{dt})_{\max }$ distribution with a consistent decrease under Nav1.5 blocking. At $t_{0}$, higher values are more widely spread in the WT than the $\Delta$ KPQ. However $(\mathrm{dF} / \mathrm{dt})_{\max }$ distribution remains anisotropic. At $\mathrm{t}_{5}$, the $\triangle \mathrm{KPQ}$ substrate reveals an area of higher upstroke velocities proximal to the base and towards the right side, whereas the area towards the apex indicates dramatically diminished $(\mathrm{dF} / \mathrm{dt})_{\max }$, as observed in the activation map. The WT map at $\mathrm{t}_{5}$ reveals slower upstrokes, with an unremarkable distribution. Look-up-table: $(\mathrm{dF} / \mathrm{dt})_{\max }\left[\begin{array}{ll}0 & 0.8\end{array}\right]$. Scale bar $=1 \mathrm{~mm}$. Ao $=$ Aorta, $\mathbf{A p}=$ Apex, $\mathbf{R}=$ Right, $\mathbf{L}=\mathrm{Left}, \mathbf{P}=\mathrm{Pacing}, \mathbf{P E}$ $=$ Pacing Electrode. $(\mathbf{b}, \mathbf{c})$. Bar graphs showing the gradual change in $(\mathbf{d F} / \mathbf{d t})_{\max }$ in longitudinal $(\mathrm{b})$ and transversal (c) directions. At $t_{0}$, the shallower upstroke in $\triangle \mathrm{KPQ}$ is on average not significantly different from its WT counterpart. The sharper decrease in the $\triangle \mathrm{KPQ}$ upstroke velocity (d) in both directions longitudinal and transversal leads to a deviation in the values between the two groups at $t_{5}$ (see text). $\mathbf{d}$. Overall decrease in (dF/dt) $\max$ between $\mathbf{t}_{0}$ and $\mathbf{t}_{5}$ exceeds $70.8 \pm 4.8 \%$ in $\triangle \mathrm{KPQ}$ (vs. $35.8 \pm 11.2 \%$ in WT) longitudinally and $59.8 \pm 7.1 \%$ transversely in $\Delta \mathrm{KPQ}$ (vs. $38.1 \pm 9.4 \%$ in WT). Annotations in figure: \# ( $p$-value $<0.0001),{ }^{* * *}(p$-value $<0.001),{ }^{* *}(p$-value $<0.01),{ }^{*}(p<0.05)$, n.s. not significant. 
A decrease in $(\mathrm{dF} / \mathrm{dt})_{\max }$ was reported for the WT group with the 10min Flecainide protocol in the previous section, where $(\mathrm{dF} / \mathrm{dt})_{\max }$ maximally dropped by $\sim 52 \%$ on average in either direction (Figure 27c). Meanwhile, it's noteworthy to recall that the $m d x$ substrate showed a significantly smaller drop in $(\mathrm{dF} / \mathrm{dt})_{\max }$ at $\mathrm{t}_{10}$ compared to its WT counterpart particularly along the transverse direction (with a total decrease of $\sim 39 \%$ ). Contrastingly, $\triangle \mathrm{KPQ}$ achieves a significantly larger decrease in $(\mathrm{dF} / \mathrm{dt})_{\max }$ compared to the WT group (at $\mathrm{t}_{5}$ ), which could indicate that the change in upstroke velocities (including the transversal ones) with Flecainide is possibly substrate dependent. Although, both $\mathrm{CV}$ and $(\mathrm{dF} / \mathrm{dt})_{\max }$ decreased with Flecainide in the WT and $\Delta \mathrm{KPQ}$, their characteristic differences do not match at control conditions, and their response to Flecainide further consolidates this contrast. While $V_{\max }$ and $V_{\min }$ are significantly larger in the WT group at $t_{0}$ compared to $\triangle \mathrm{KPQ}$, their corresponding $(\mathrm{dF} / \mathrm{dt})_{\max }$ are almost similar (no significant difference). Similarly, the effects at $t_{5}$ are reversed: the velocities become almost comparable between the two groups in either direction, whereas their corresponding upstroke velocities considerably differ. Indeed in these two groups, $\mathrm{CV}$ and $(\mathrm{dF} / \mathrm{dt})_{\max }$ appear to behave characteristically different with or without Flecainide.

\subsubsection{Flecainide Exacerbates Intrinsic Heterogeneity in $\triangle K P Q$}

One of the arrhythmogenic mechanisms proposed for the LQTS substrate is amplification of the SDR, which when present in either early or late phases of the AP can lead to the development of a variety of arrhythmias ${ }^{403}$. Amplification of intrinsic heterogeneity together with premature impulses could principally underlie the mechanistic trigger for ventricular arrhythmias in cardiac pathologies such as heart failure ${ }^{271}$ and "torsade de pointes" in LQTS3 ${ }^{406}$. Gene-specific therapy includes a 2-hit approach: The first is linking the genetic mutation to the ionic channel dysfunction, whereas the second is identifying the pharmacological agents that can modulate the mutated channels in a differential manner 281 .

The response of arrhythmogenic LQTS3 substrate to AADs remains the subject of strenuous debate. Schwartz et al. and others reported that $\mathrm{Na}_{\mathrm{V}} 1.5$ blocker Mexiletine (a Class IB AAD) is more effective in shortening the QT-interval in LQTS3 patients than other LQTS due to $\mathrm{K}^{+}$-channel mutations ${ }^{282,574 .}$ Shimizu et al. showed that a $\mathrm{K}^{+}$channel opening agent (Nicorandil) abbreviated the QT-interval and the monophasic AP duration in LQTS1 patients ${ }^{281}$. Shortening of QT interval isn't necessarily harmonious with decreasing or preventing arrhythmia risk in these patients ${ }^{281}$, since a direct validation of the QT interval as a measure of APD spatial dispersion is still lacking 575 . On the other hand, Nav1.5 blocking agents haven't been reported to be ineffective in treating LQTS3 only576, but also dangerously proarrhythmic ${ }^{570}$, sometimes despite a normalized QT-interval on ECG ${ }^{577}$. Hence normalizing the QT-interval is not necessarily congruent with decreased SCD risk.

We show in the following results that exaggerated SDR is predominantly observed in the $\triangle \mathrm{KPQ}$ mouse upon exposure to Flecainide at clinically valid concentrations, which could make it a plausible mechanism in increasing arrhythmia susceptibility in LQTS3 patients managed with Nav1.5 blockers. 
a

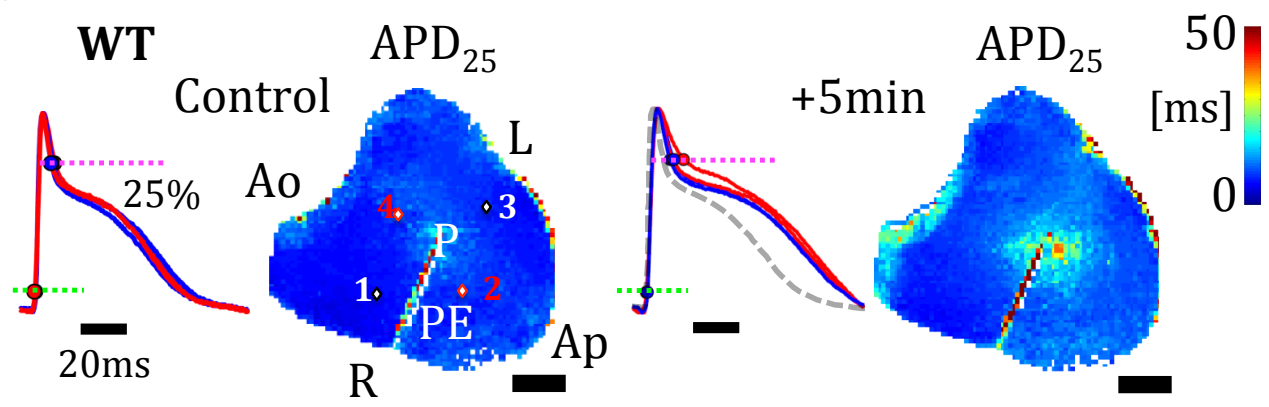

$\triangle \mathrm{KPQ}$

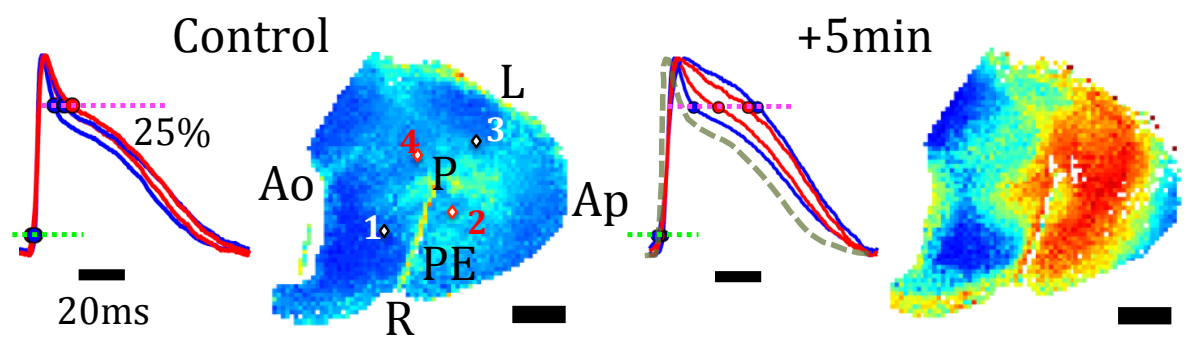

$\mathrm{b}$

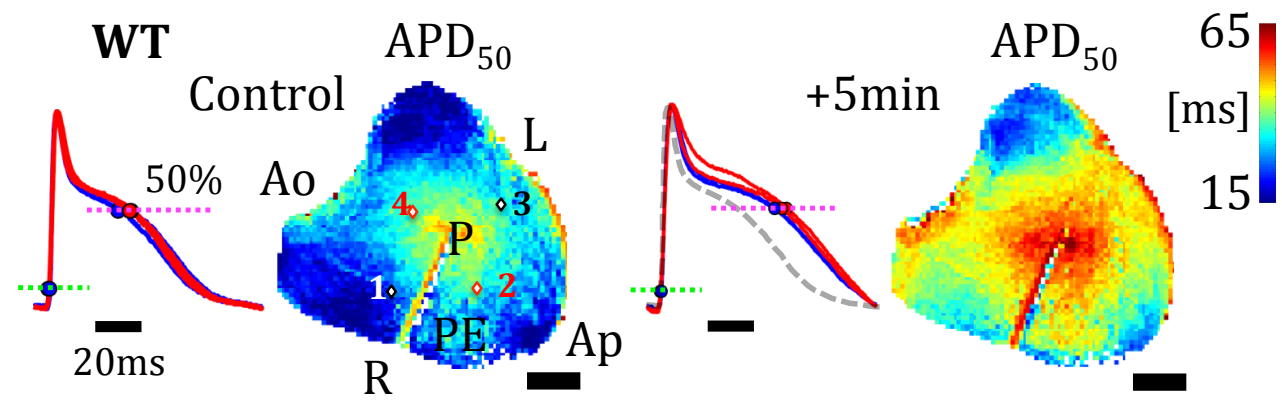

$\triangle \mathrm{KPQ}$

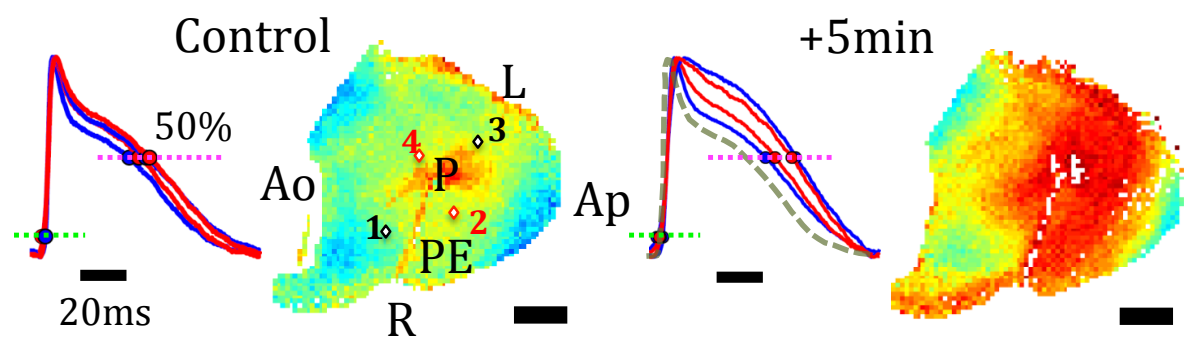

Figure 39. APs and $\mathrm{APD}_{25}(\mathrm{a}), \mathrm{APD}_{50}$ (b) dispersion maps for WT and $\triangle \mathrm{KPQ}$ at $\mathrm{t}_{0}$ and $\mathrm{t}_{5}$ of Nav1.5 blocking. a. Symmetry breaking at t5 with multiple zones of abbreviated, intermediate, and prolonged APs across the LV epicardium in $\triangle \mathrm{KPQ}$. APD $25, \mathrm{WT}$ map prolongs homogeneously, while the APs remain superimposable after $5 \mathrm{~min}$ of exposure to Flecainide. The initial $\mathrm{APD}_{25}$ configuration in the $\triangle \mathrm{KPQ}$ substrate cannot be considered homogeneous (compared to the WT map above). Some heterogeneity component exists at $t_{0}$, which might have been amplified in the presence of Flecainide, giving rise to the multiple distinctive $\mathrm{APD}_{25}$ zones in the dispersion map. b. Symmetry breaking remains identifiable for $\mathrm{APD}_{\mathbf{5 0}}$ at $\mathbf{t}_{\mathbf{5}}$ in the $\triangle \mathrm{KPQ}$ epicardium. The effects observed for $\mathrm{APD}_{50}$ are similar to the ones described at $25 \%$ repolarization. The circular distribution of APD50,wT is maintained at $\mathrm{t}_{5}$, however associated with a homogeneous prolongation of APD values. The pattern observed at $t_{5}$ for the APD $50, \Delta \mathrm{KPQ}$ map differs from the APD $25, \Delta \mathrm{KPQ}$ by the amplitude of the gradient separating the lower and upper ranges of APD 50 , which is limited to $\sim 20 \mathrm{~ms}$, as shown also by the APs on the left. Annotations in figure: Map scale bar $=1 \mathrm{~mm}$, AP scale bar $=20 \mathrm{~ms}, \mathbf{A o}=\mathrm{Aorta}, \mathbf{A p}=\mathrm{Apex}$, $\mathbf{R}=$ Right, $\mathbf{L}=$ Left, $\mathbf{P E}=$ Pacing electrode, $\mathbf{P}=$ Point stimulation. 
In Figure 39, APs and their corresponding APD dispersion maps $\left(\mathrm{APD}_{25}(\mathrm{a}), \mathrm{APD}_{50}(\mathrm{~b})\right)$ for WT and $\Delta \mathrm{KPQ}$ at $\mathrm{t}_{0}$ and $\mathrm{t}_{5}$ of Flecainide $[1 \mu \mathrm{M}]$ are shown. As previously described, the same mask of the LV free wall was repeatedly used for the same prep to reproduce the maps at different time points of exposure to the drug. The APs on the left are chosen from the located points in the maps where white dots reside along the longitudinal direction with corresponding blue colored APs, and red dots along the transversal direction with corresponding red colored APs. Measurements were conducted at $t_{0}, t_{1}, t_{3}$ and $t_{5}$, but only $t_{0}$ and $t_{5}$ are shown in the figure. The morphological changes in OAPs are tracked by superimposing the APs at $t_{5}$ over their control AP (in dotted line). APwT at $t_{0}$ perfectly overlap, and their corresponding $\mathrm{APD}_{25}$ map further confirms the homogeneity of the cardiac tissue under these conditions. The map's associated histograms are shown in Figure 40a (leftmost panel). The unimodal distribution of $\mathrm{APD}_{25, \mathrm{WT}}$ values are centered around $7.2 \mathrm{~ms}$ with a dispersion of $\triangle \mathrm{APD}_{25, \mathrm{WT}}=7.9 \mathrm{~ms}$. The average dispersion in the WT group ( $\mathrm{n}=4$, Figure 41$)$ under control conditions is $8.7 \pm 1.7 \mathrm{~ms}$. In contrast, the $\mathrm{APD}_{25, \Delta \mathrm{KPQ}}$ map (Figure $39 \mathrm{a}$, below left) shows patchy regions of more prolonged APs across the LV free wall (lighter blue), with reduced APs found mainly at the basal side. The distribution of the $\mathrm{APD}_{25, \Delta \mathrm{KPQ}}$ values is centered around $13.5 \mathrm{~ms}$, has a wider base than its WT counterpart with $\triangle \mathrm{APD}_{25, \Delta \mathrm{KPQ}}=16.2 \mathrm{~ms}$ (Figure $40 \mathrm{a}$, right). In conclusion, at $t_{0}$ when Flecainide is not yet in perfusion, not only $A P D_{25, \Delta K P Q}$ in terms of values is significantly larger (Figure $42 \mathrm{a}$, right and left panels), but also $\triangle \mathrm{APD}_{25, \triangle \mathrm{KPQ}}(\mathrm{n}=4)$ is significantly wider than its WT counterpart $\left(\triangle \mathrm{APD}_{25, \mathrm{WT}}=8.7 \pm 1.7 \mathrm{~ms} ; \Delta \mathrm{APD}_{25, \Delta \mathrm{KPQ}}=14.9 \pm 4.1 \mathrm{~ms}, \quad p\right.$-value $<0.05$, Figure 41$)$, corresponding to the appearance of sketchy clusters of longer APDs in the mutated substrate.

At $t_{5}$, the $\mathrm{AP}_{\mathrm{WT}}$ have shallower upstrokes with a decreased notch, followed by an increase in the APD mainly at later repolarization phases. While the map shows a homogenous prolongation in $\mathrm{APD}_{25 \text {,WT }}$ values, the histograms at $t_{5}$ (Figure $40 \mathrm{a}$, left) conserve a high peak centered at $10.2 \mathrm{~ms}$ with a longer tail, causing an increase in base width $\left(\triangle \mathrm{APD}_{25, \mathrm{WT}}=17.4 \mathrm{~ms}\right.$ in this example). On average, $\Delta \mathrm{APD}_{25, \mathrm{WT}}$ increased in total of $\sim 120 \%$ to reach a value of $19.7 \pm 5.1 \mathrm{~ms}$ by $t_{5}$ (Figure 41 ). The response of the $\triangle \mathrm{KPQ}$ tissue to Flecainide at $\mathrm{t}_{5}$ however appears more dramatic, with multiple adjacent regions of shorter, intermediate, and prolonged $\mathrm{APD}_{25}$ allocated between the base and apex of the LV free wall (Figure 39a, bottom, right). On the left side of the map, the APs selected from 4 different locations within the ventricle display gradual loss of their notch, followed by a proportional increase in the APD. By looking at the position of each of the APs, it becomes obvious that epicardial cells that once displayed almost superimposable APs at $t_{0}$ (for instance locations 1 and 3 , along the longitudinal direction) are separated by an $\mathrm{APD}_{25}$ gradient exceeding $30 \mathrm{~ms}$ after $5 \mathrm{~min}$ exposure to Flecainide. A direct comparison of the $\mathrm{APD}_{25, \triangle \mathrm{KPQ}}$ maps at $t_{0}$ and $t_{5}$ reveals the possibility that the patterns formed at $t_{5}$ may be the result of amplification of intrinsic heterogeneity found within the mapped epicardial layer, indicated by the patchy arrangement of $\mathrm{APD}_{25}$ initially found at $\mathrm{t}_{0}$. Nevertheless, it's not yet clear whether the patterns that appeared with Flecainide can potentially be predicted from the initial conditions at $t_{0}$. The histograms of the corresponding map no longer display any particular peak and nicely span a whole range of values with a dispersion $\triangle \mathrm{APD}_{25, \Delta K \mathrm{KQ}}=35.7 \mathrm{~ms}$ (Figure 40a, right). From the bar graphs in Figure 41, it becomes evident that $\mathrm{APD}_{25}$ dispersion is significantly larger in the $\triangle \mathrm{KPQ}$ substrate at either $\mathrm{t}_{0}$ or $\mathrm{t}_{5}$, with an increase of $\sim 120 \%$ and $\sim 135 \%$ in $\triangle \mathrm{APD}_{25, \mathrm{WT}}$ and $\triangle \mathrm{APD}_{25, \Delta \mathrm{KPQ}}$ respectively between $\mathrm{t}_{0}$ and $\mathrm{t}_{5}$ (Figure 43 ). 
The effects observed in $\mathrm{APD}_{25}$ are translated to later phases of the AP (Figure $39 \mathrm{~b}$ ). The circular pattern with slightly protracted APDs at the site of pacing with a smoothing of APD value towards the boundaries persists for the WT APD 50 map at $\mathrm{t}_{0}$ with an additional homogeneous prolongation of the APD in all pixels at $t_{5}$. In this example under control conditions, dispersion of $\mathrm{APD}_{50, \mathrm{wT}}$ is calculated to be $\triangle \mathrm{APD}_{50, \mathrm{WT}}=34.8 \mathrm{~ms}$ (Figure $40 \mathrm{~b}$, leftmost), which further increases to $50.3 \mathrm{~ms}$ at $\mathrm{t}_{5}$. With the current configuration of $A P D_{50, \Delta K P Q}$ at $t_{0}$, the histograms indicate a unimodal distribution of values centered at $41.5 \mathrm{~ms}$ and with a dispersion $\triangle \mathrm{APD}_{50, \Delta \mathrm{KPQ}}=35.8 \mathrm{~ms}$. On average, the dispersion of $\mathrm{APD}_{50}$ values is not found to be significantly different between WT and $\triangle \mathrm{KPQ}$ at control conditions. Only after the development of symmetry breaking in the $\triangle \mathrm{KPQ}$ substrate at $\mathrm{t}_{5}$, that this difference becomes statistically significant (Figure 41). Counterintuitively, the dispersion in APD50 values are considerably larger in the WT group, despite the presence of symmetry breaking exclusively in the $\triangle \mathrm{KPQ}$ hearts at $\mathrm{t}_{5}$, with $\Delta \mathrm{APD}_{50, \mathrm{WT}}=47.2 \pm 5.0 \mathrm{~ms} ; \Delta \mathrm{APD}_{50, \Delta \mathrm{KPQ}}=36.3 \pm 7.2 \mathrm{~ms} \quad(p$-value $<0.05)$. Importantly, by looking at the bar graphs (Figure 41 ), $\triangle \mathrm{APD}_{50, \mathrm{wT}}$ undergoes $\sim 45 \%$ increase in width between $t_{0}$ and $t_{5}$, vs. a slight increase of less than $5 \%$ in the $\Delta \mathrm{APD}_{50, \Delta \mathrm{KPQ} \text {. }}$

Figure 40. Histograms extracted from APD maps at different repolarization rates $(25 \%$ and $50 \%)$ and $(\mathrm{dF} / \mathrm{dt})_{\max }$ for WT and $\triangle \mathrm{KPQ}$, at $\mathrm{t}_{0}$ and $\mathrm{t}_{5}$ of Nav1.5 blocking. a. APD25 peaks at $7.2 \mathrm{~ms}$ and $13.5 \mathrm{~ms}$ at to for WT and $\triangle \mathrm{KPQ}$ respectively. $\triangle \mathrm{APD}_{25, \Delta \mathrm{KPQ}}$ approximates to $16.2 \mathrm{~ms}$ at $\mathrm{t}_{0}$ and increases to $35.7 \mathrm{~ms}$ at $t_{5}$. This dispersion is considerably larger than the one calculated for the WT (evident from the substantial widening of the distribution base), where $\triangle \mathrm{APD}_{25, \mathrm{WT}}=17.4 \mathrm{~ms}$. The loss of the unimodal distribution in the mutated group at $t_{5}$, which also spans a whole range of $\mathrm{APD}_{25}$ values, accounts for the multiple adjacent zones of notably different APD across the LV free wall. b. APD 50 distribution at $t_{0}$ is wider in the WT group in this particular example, where considerably more pixels returned APD values in the lower range. APD50,WT peaks around $28.1 \mathrm{~ms}$, with a $\triangle \mathrm{APD} \mathrm{D}_{50, \mathrm{WT}}=34.8 \mathrm{~ms}$. The latter further increases to $50.3 \mathrm{~ms}$ at $\mathrm{t}_{5}$. Despite the WT being devoid of symmetry breaking, $\triangle \mathrm{APD}_{50, \mathrm{WT}}$ is notably larger than the one calculated in the $\triangle \mathrm{KPQ}$ heart at $\mathrm{t}_{5}$, where symmetry breaking actually takes place $\left(\triangle A P D_{50, \Delta \mathrm{KPQ}}=35.7 \mathrm{~ms}\right)$. c. Upstroke velocities distribution of the maps in Figure 38. The bimodal distribution of WT $(\mathrm{dF} / \mathrm{dt})_{\max }$ at $\mathrm{t}_{0}$ is compressed into one larger peak at $t_{5}$, due to loss of high upstroke values with Flecainide. A stronger shift to the left is noticeable for the histograms of $\triangle \mathrm{KPQ}$ with Flecainide, indicating a considerable loss of previously existing higher $(\mathrm{dF} / \mathrm{dt})_{\max }$ at $\mathrm{t}_{0}$.
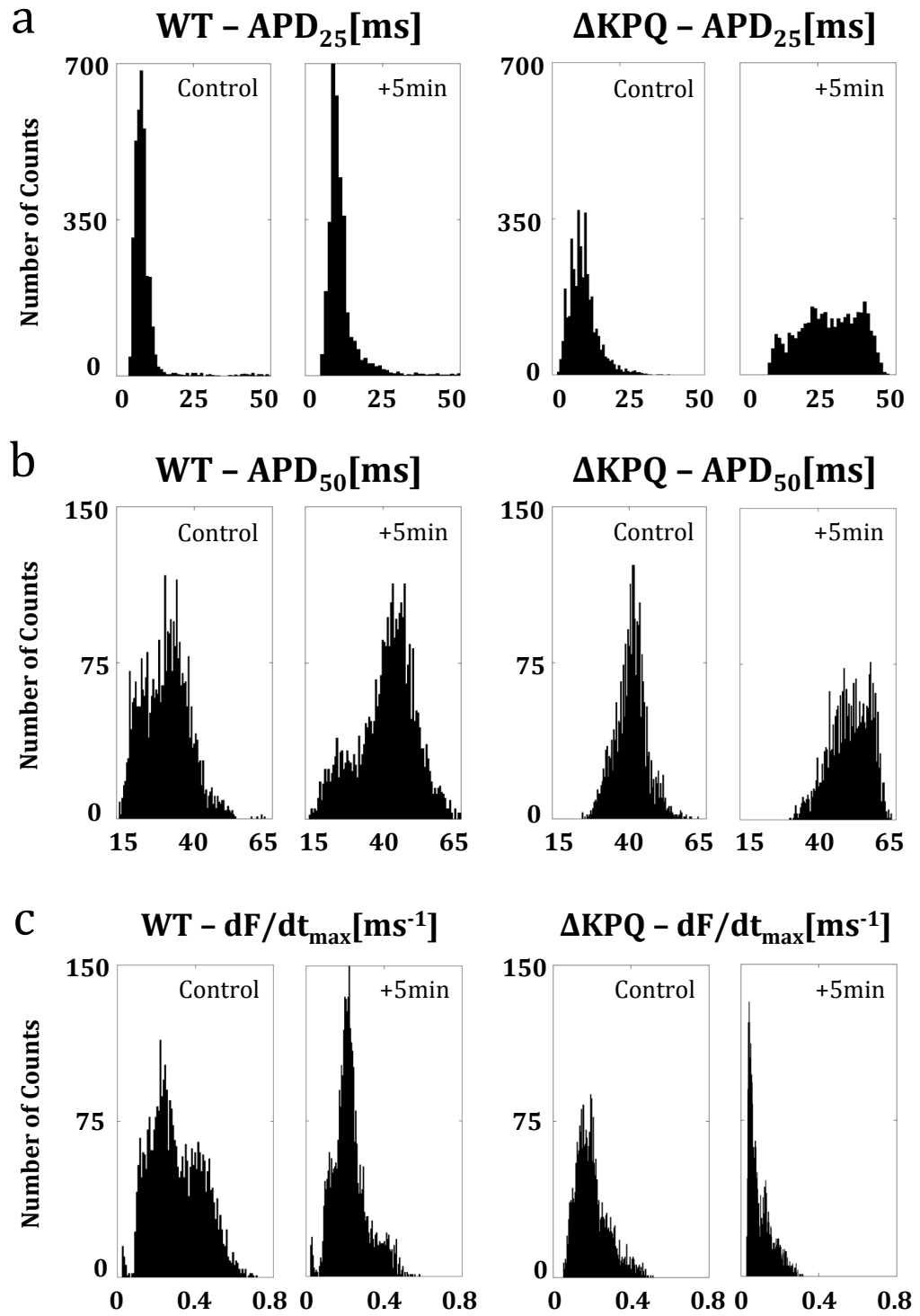


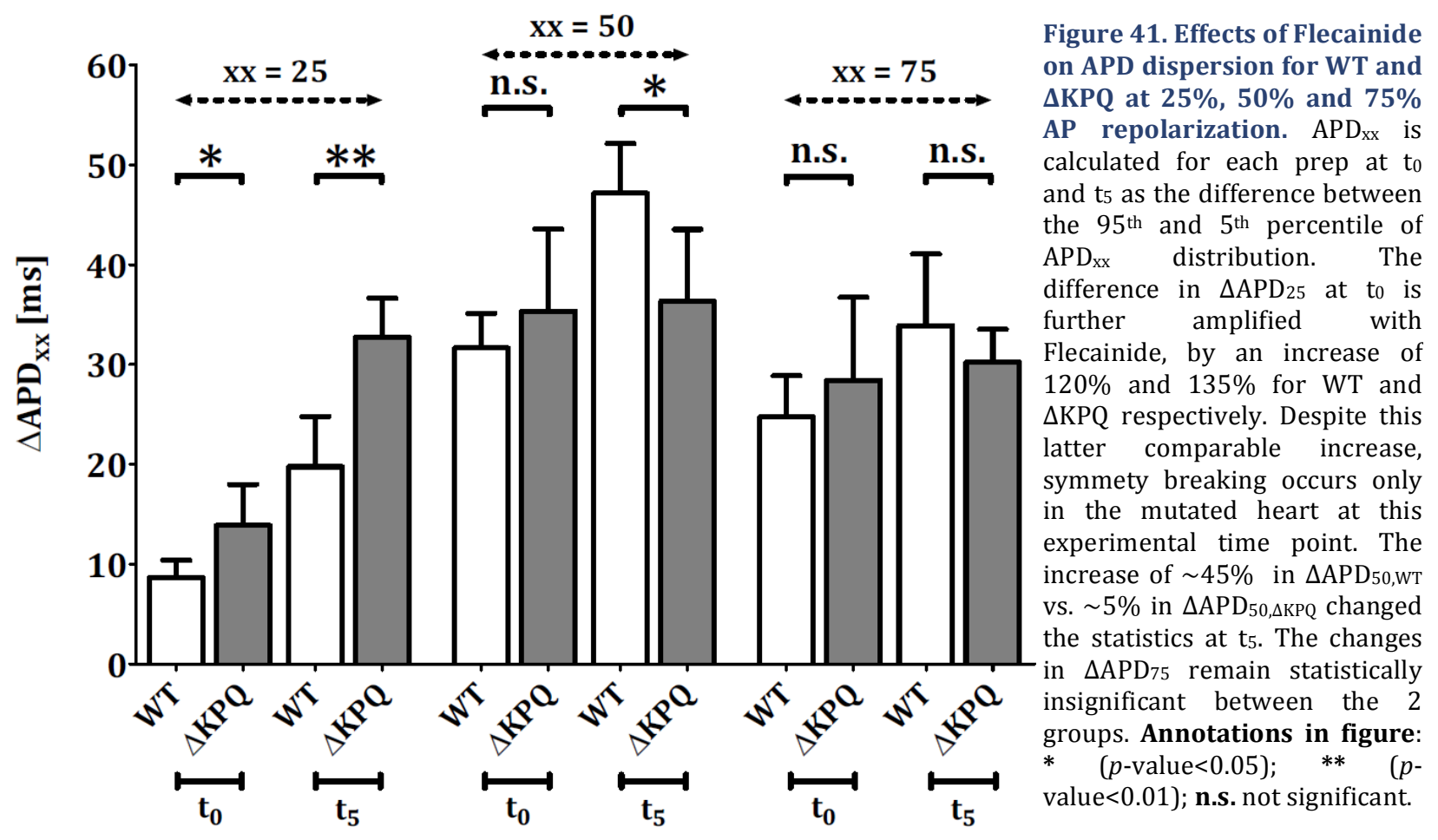

While some heterogeneity is still observed at repolarization levels beyond 50\%, it's less prominent than the one detected at $25 \%$ and $50 \%$ (maps not shown here). The dispersion of $\mathrm{APD}_{75}$ values (Figure 41$)$ is not significantly different between the two groups at $t_{0}\left(\triangle \mathrm{APD}_{75, \mathrm{WT}}=24.6 \pm 4.3 \mathrm{~ms}\right.$; $\Delta \mathrm{APD}_{75, \Delta \mathrm{KPQ}}=28.4 \pm 8.3 \mathrm{~ms} ; p$-value=0.44), nor at $\mathrm{t}_{5}\left(\Delta \mathrm{APD}_{75, \mathrm{WT}}=33.9 \pm 7.2 \mathrm{~ms} ; \Delta \mathrm{APD}_{75, \Delta \mathrm{KPQ}}=\right.$ $30.2 \pm 3.3 \mathrm{~ms} ; p$-value $=0.39$ ).

In addition to dispersion analysis, APDs at different levels of repolarization were analyzed and compared between WT and $\triangle \mathrm{KPQ}$ at different time points of Flecainide exposure (Figure 42). While $A P D_{25}, A P D_{50}$ and $A P D_{75}$ show statistically significant differences at control conditions $\left(t_{0}\right)$ between the experimental groups, exposure to Flecainide caused a consistent prolongation of all APDs abolishing at almost all time points the existing differences between them.

The effect AP abbreviation or prolongation has on spatial dispersion has not been quantitatively well determined. It's strongly believed however that asymmetrical AP prolongation in the heart leads to increased spatial dispersion, enhancing medium susceptibility for arrhythmic activity. From my current data, the correlation between $\mathrm{APD}_{\mathrm{xx}}$ and $\triangle \mathrm{APD}_{\mathrm{xx}}$ ( $\mathrm{xx}$ standing for any percentage repolarization) could by itself provide a quantitative indicator for the possible occurrence of symmetry breaking in the ventricle. Following the previous analysis of $\triangle$ APD (Figure 41), APD values were compared between $\mathrm{WT}(\mathrm{N}=8)$ and $\triangle \mathrm{KPQ}(\mathrm{N}=8) . \mathrm{APD}_{25}$ (along longitudinal and transversal directions, Figure 42a), APD 50 (longitudinal only, Figure 42b), APD 75 (longitudinal only, Figure 42c) bar graphs are displayed. The $\triangle \mathrm{KPQ}$ heart for all APDs at $\mathrm{t}_{0}$ shows significantly larger values than its WT counterpart. For instance, $\mathrm{APD}_{25, \mathrm{WT}}$ (Long.) $=6.0 \pm 1.0 \mathrm{~ms}, \mathrm{APD}_{25, \Delta \mathrm{KPQ}}$ (Long.) $=$ $10.0 \pm 4.1 \mathrm{~ms}$ ( $p$-value $<0.05$ ). The transversal $\mathrm{APD}_{25}$ are particularly shown here because of their distinct changes with Flecainide (when comparing the groups) relatively to the longitudinal values, which is not the case for the $\mathrm{APD}_{50}$ or $\mathrm{APD}_{75}$ therefore only longitudinal values are displayed in the figure. Similarly, $\mathrm{APD}_{50, \mathrm{WT}}=27.7 \pm 5.6 \mathrm{~ms}, \mathrm{APD}_{50, \Delta \mathrm{KPQ}}=36.1 \pm 6.1 \mathrm{~ms}(p$-value $<0.05)$, whereas $\mathrm{APD}_{75, \mathrm{WT}}=$ $46.7 \pm 6.9 \mathrm{~ms}, \mathrm{APD}_{75, \Delta \mathrm{KPQ}}=54.2 \pm 5.7 \mathrm{~ms}(p$-value $<0.05)$. 
As all APDs prolong with Flecainide in both groups (WT and $\triangle \mathrm{KPQ}$ ), the maximal increase is naturally observed in $\mathrm{APD}_{25}$, being the initially smallest entity measured. The total increase in $\mathrm{APD}_{25}$ along both directions is not only compared among the groups, but also contrasted to the increase in $\Delta \mathrm{APD}_{25}$ between $\mathrm{t}_{0}$ and $\mathrm{t}_{5}$ (Figure 43). To recall from previous experiments done on the $m d x$ heart with its WT control, symmetry breaking occurs only in the WT heart at $\mathrm{t}_{10}$ but didn't take place in the $m d x$ heart. The total increase (percentage wise) in $\mathrm{APD}_{25}$ was significantly larger in WT (summarized in Figure 43) in either direction compared to the changes detected in the $m d x$. Contrastingly, comparing the percentage prolongation in $\mathrm{APD}_{25}$ at $\mathrm{t}_{5}$ between $\triangle \mathrm{KPQ}$ and WT, the latter has shown a considerably larger increase along the longitudinal direction (the transversal $\mathrm{APD}_{25}$ increase is $100 \%$ for either group), yet symmetry breaking took place in the $\triangle \mathrm{KPQ}$ ventricle and not the WT. Therefore, it seems that the extent of APD prolongation between control and end of treatment conditions alone cannot predict loss of symmetry in APD patterns.
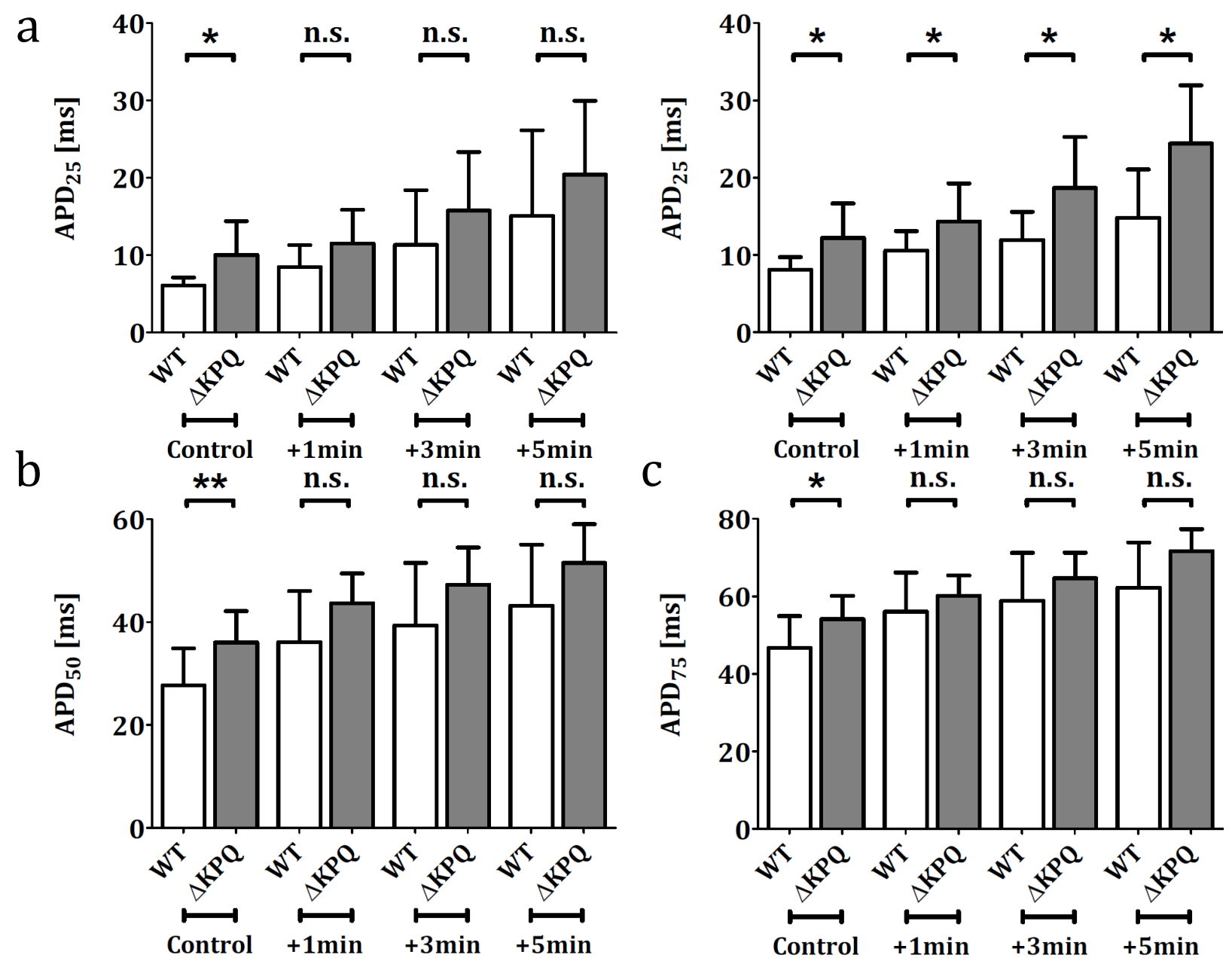

Figure 42. Bar graphs showing the progression of $A P D_{25}(a), A P D_{50}$ (b) and $A P D_{75}$ (c) with Flecainide in both WT $(\mathrm{N}=8)$ and $\triangle \mathrm{KPQ}(\mathrm{N}=\mathbf{8})$. Only for the $\mathrm{APD}_{25}$, both longitudinal (left) and transversal (right) are shown. For the remaining $\mathrm{APD}_{50}$ and $\mathrm{APD}_{75}$, bar graphs contain values picked along the longitudinal direction only. At initial conditions, the $\triangle \mathrm{KPQ}$ substrate shows a consistent prolongation of its APDs compared to its WT counterpart. As Flecainide proceeds to 5min," the significant difference between the groups is abolished except in $\mathrm{APD}_{25}$ (transversally), where the APDs remain significantly higher in the mutated at all experimental time points. The overall increase in APD values between $t_{0}$ and $t_{5}$ can be summarized as such: (Long.) $150 \%$ in $\mathrm{APD}_{25, \mathrm{WT}}$ vs. $105 \%$ in APD $25, \Delta \mathrm{KPQ}$. (Trans.) $100 \%$ in both $\mathrm{APD} 25, \mathrm{WT}$ and $\mathrm{APD}_{25, \Delta \mathrm{KPQ}} .55 \%$ in $\mathrm{APD}_{50, \mathrm{WT}}$ vs. $43 \%$ in $\mathrm{APD}_{50, \Delta \mathrm{KPQ} .} 33 \%$ in $\mathrm{APD}_{75, \mathrm{WT}} \mathrm{vs.} 30 \%$ in $\mathrm{APD}_{75, \Delta \mathrm{KPQ}}$ 
As mentioned earlier, symmetry breaking has occurred in 8 out of 10 WT hearts at $10 \mathrm{~min}$ of Flecainide exposure, and in 4 out of 4 experiments in $\triangle \mathrm{KPQ}$ hearts at only half that time. None of 7 $m d x$ experiments has exhibited such a heterogeneous distribution at any experimental time. If this pattern formation is a generic property of the cardiac tissue that manifests itself at specific experimental and electrophysiological conditions, one or several functional parameters combined could bring forward particular requirements that might expedite its appearance.

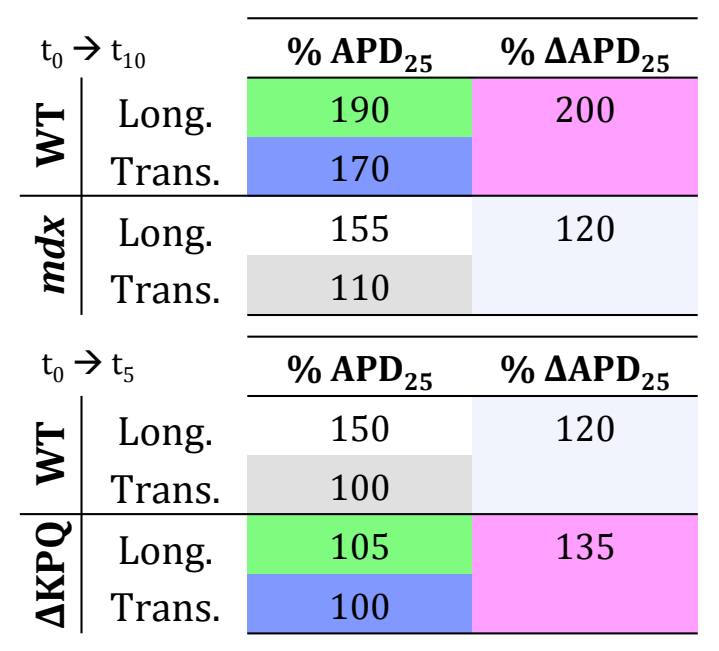

Figure 43. Table summarizing the percentage increase in $A P D_{25}$ between $t_{0}$ and $t_{10}$ of Flecainide exposure for WT and $m d x$, and between $t_{0}$ and $t_{5}$ for $\mathrm{WT}$ and $\triangle \mathrm{KPQ}$; along with the corresponding increase in dispersion $\left(\triangle A P D_{25}\right)$. From $t_{0} \rightarrow t_{10}$ : the $m d x$ heart shows a more restrained prolongation of $\mathrm{APD}_{25}$ in either direction compared to the WT. More importantly unlike the $m d x$, the increase in $\triangle \mathrm{APD}_{25, \mathrm{WT}}$ exceeds the total prolongation in $\mathrm{APD}_{25}$ itself in either direction. From $\mathbf{t}_{\mathbf{0}} \rightarrow \mathbf{t}_{5}$ : Although $\mathrm{APD}_{25, \mathrm{WT}}$ shows a larger prolongation with Flecainide than the $\triangle \mathrm{KPQ}$, the total increase in dispersion was inferior to the total prolongation in $\mathrm{APD}_{25, \mathrm{WT}}$. Despite a more constricted increase in $\mathrm{APD}_{25, \Delta \mathrm{KPQ}}$ symmetry breaking erupted in the $\triangle \mathrm{KPQ}$. Only in substrates where the global increase in $\triangle \mathrm{APD}_{25}$ (pink cells) exceeds the total prolongation of its respective APDs (green, blue), symmetry breaking flared up.

Such a symmetry breaking pattern is likely to be arrhythmogenic, especially if a steep gradient in repolarization exists over very short distances, as in the mouse heart experiments presented in this thesis work. Targeting Nav1.5 is fundamental in Class I drugs, the mechanism of action of which has long been considered well understood and fairly straight forward. The race to understand the proarrhythmic effects of AADs has started more than two decades ago after the groundbreaking results of CAST and till now, the mechanisms of arrhythmia trigger and sustenance are not fully elucidated. These results map a detrimental side effect of Flecainide of potentially strong clinical significance: clinically valid concentrations of Flecainide were sufficient to induce a steep spatial dispersion of repolarization in small hearts, where electrotonic conduction is normally sufficiently high to smooth out functional heterogeneities. Such patterns formation in larger hearts, where more structural heterogeneity prevails, could be several orders of magnitude more dangerous and lethal. In cardiac tissues, where electrical excitation and conduction across coupled cells is maintained by $\mathrm{Na}^{+}$, pharmacological interventions through $\mathrm{Na}_{v} 1.5$ blocking have the potential to amplify cardiac instability by decoupling universal parameters of excitability and conductivity, including weakening electrical depolarization, slowing conduction, increasing repolarization time and finally splitting the intact tissue into adjacent zones of steep repolarization gradients. 
a $\Delta \mathrm{KPQ}-\mathrm{t}_{0}-$ Slower impulse conduction

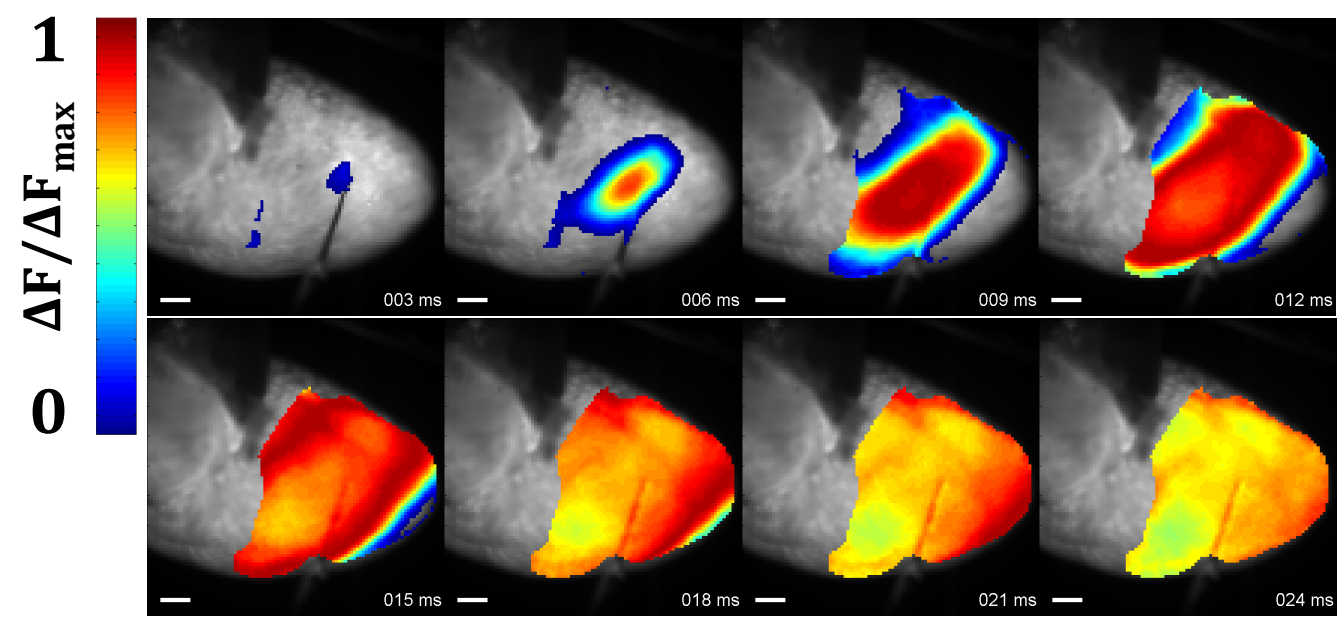

b

Figure 44. Activation (a) followed by repolarization (b) from intact epicardium of a $\triangle \mathrm{KPQ}$ mouse heart in the absence of Flecainide. From a point stimulation (pacing electrode aiming at the center of the LV free wall), an elliptical wave propagates towards the boundaries. The repolarization pattern is not homogeneous compared to the WT example shown in the Methods' section. Patchy distribution of prolonged APDs prevents a congruent repolarization and increases spatial-functional heterogeneity. Scale bar = $1 \mathrm{~mm}$.

\section{$\Delta \mathrm{KPQ}-\mathrm{t}_{0}-$ Inhomogeneous repolarization}

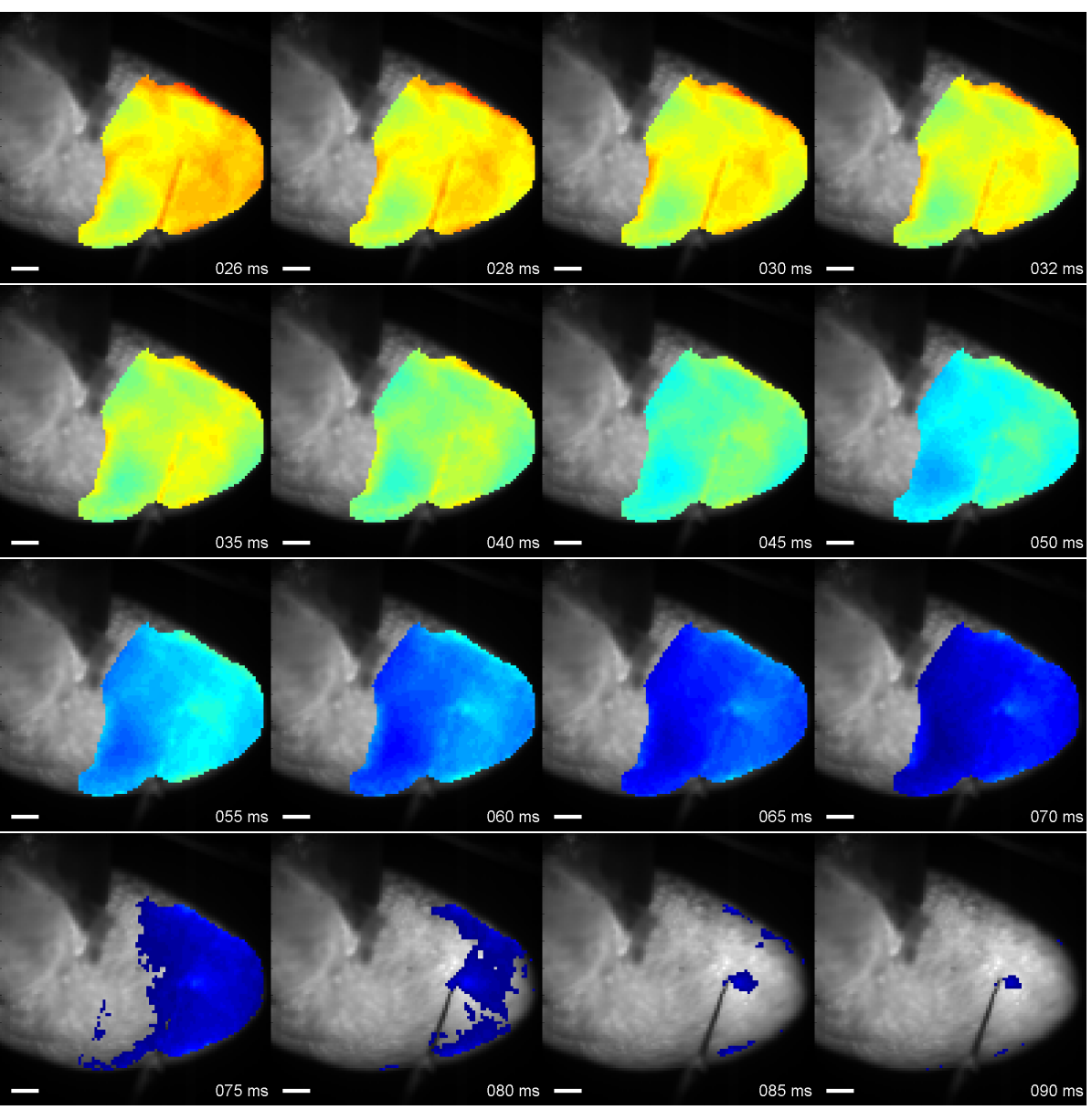




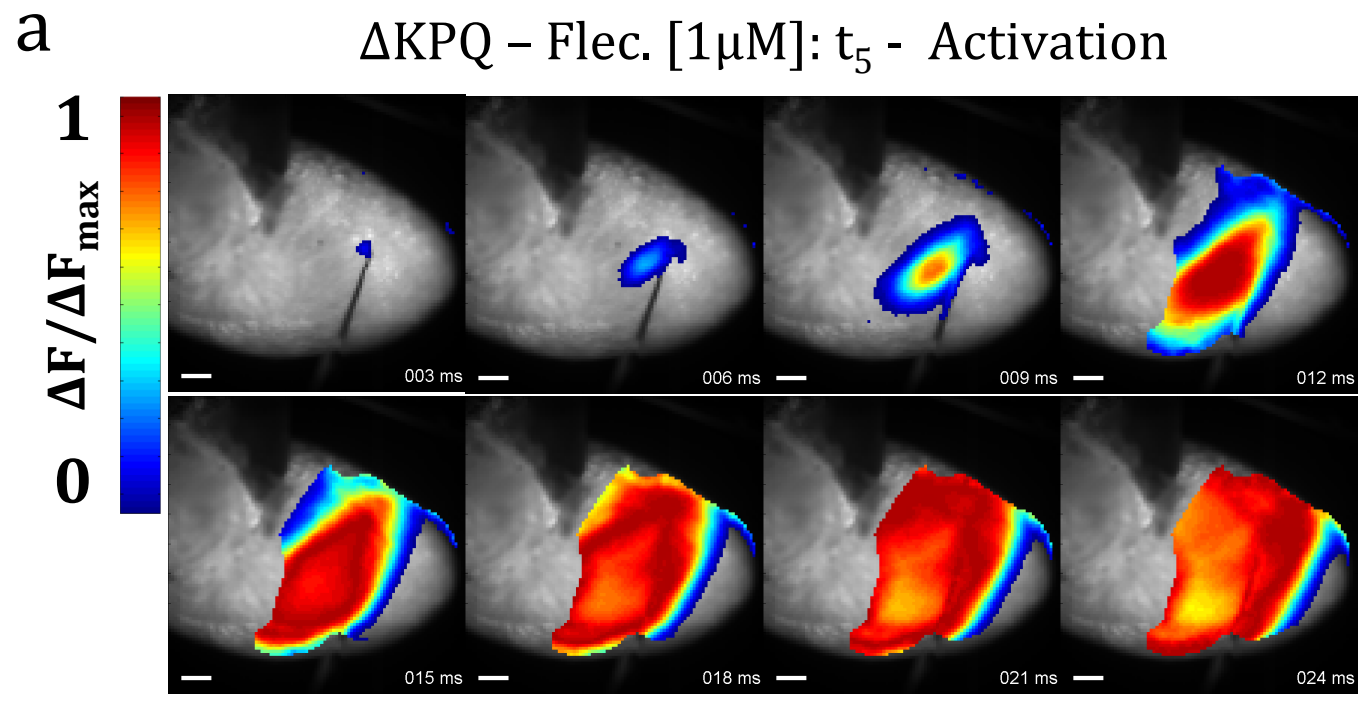

b

Figure 45. Activation (a) followed by repolarization (b) from intact epicardium of a $\triangle \mathrm{KPQ}$ mouse heart after $5 \mathrm{~min}$ of Flecainide exposure. From a point stimulation (pacing electrode remaining at exact same position as in Figure 44), activation is slower and asymmetrical on the apicobasal axis. Multiple adjacent zones of inconsistent repolarization properties amplify spatial-temporal heterogeneity and SDR, rendering the tissue a highly susceptible substrate for arrhythmia. Scale bar $=1 \mathrm{~mm}$.

\section{Expatiated Repolarization Abnormalities}

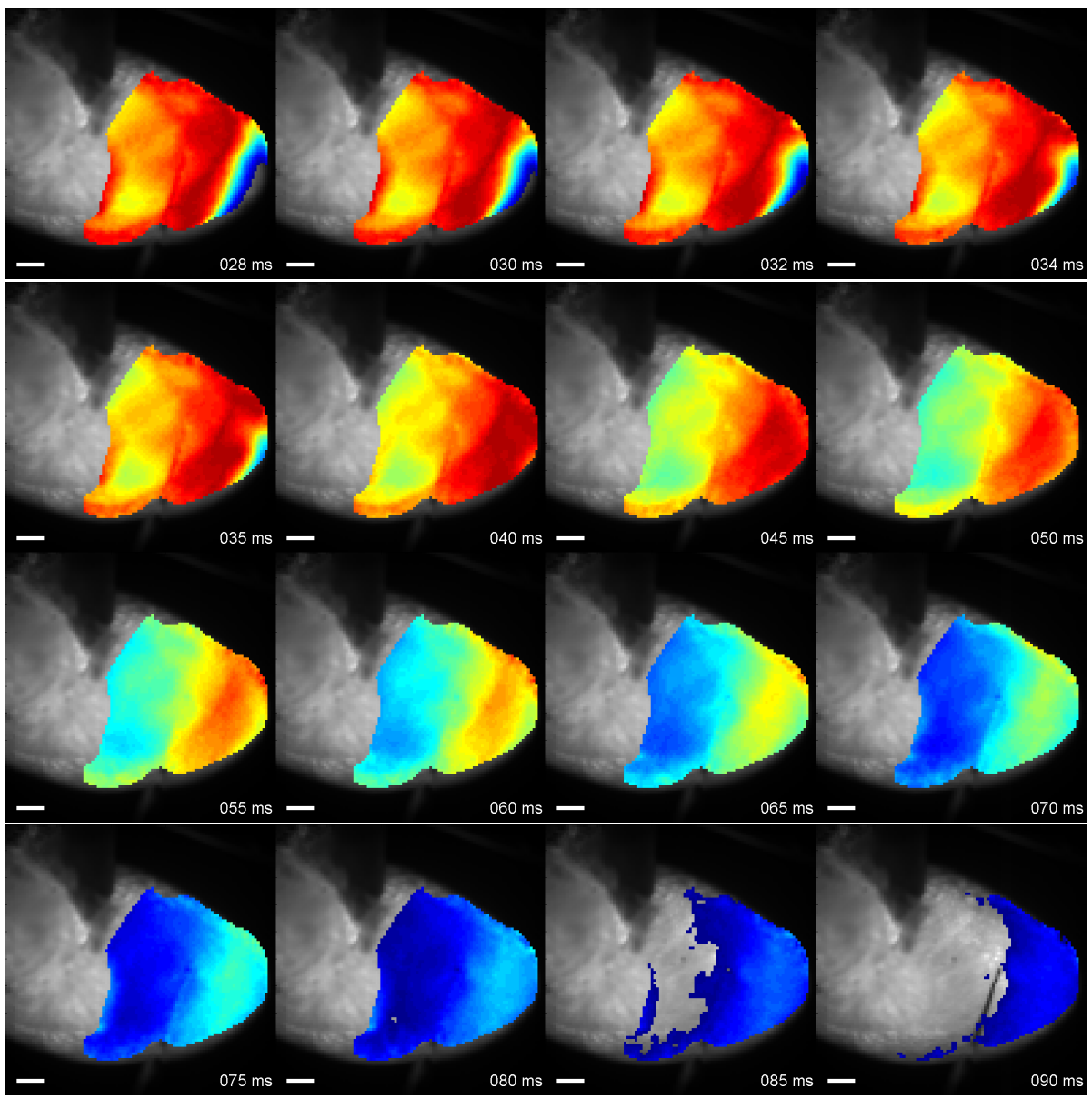




\begin{tabular}{|c|c|c|c|c|c|}
\hline & & $\mathrm{x}$-axis & $y$-axis & Slope \pm error & $\begin{array}{c}\text { Correlation } \\
\text { coef. }\left(\mathbf{R}^{2}\right)\end{array}$ \\
\hline & & LSEF & $\mathrm{PF}$ & $0.55 \pm 0.17$ & 0.41 \\
\hline & 3 & LESF & $\mathrm{PF}$ & $0.59 \pm 0.20$ & 0.41 \\
\hline 2 & & $\mathrm{PF}$ & $\mathrm{AF}$ & $0.97 \pm 0.14$ & 0.75 \\
\hline$\overline{\mathfrak{z}}$ & & LSEF & $\mathrm{PF}$ & $0.67 \pm 0.15$ & 0.58 \\
\hline 氕 & $\mathfrak{\Xi}$ & LESF & $\mathrm{PF}$ & $0.75 \pm 0.14$ & 0.64 \\
\hline 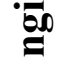 & & $\mathrm{PF}$ & $\mathrm{AF}$ & $0.97 \pm 0.12$ & 0.82 \\
\hline$\beth$ & T & LSEF & $\mathrm{PF}$ & $0.87 \pm 0.05$ & 0.90 \\
\hline & dै & LESF & $\mathrm{PF}$ & $0.89 \pm 0.05$ & 0.92 \\
\hline & $z$ & $\mathrm{PF}$ & $\mathrm{AF}$ & $0.99 \pm 0.03$ & 0.97 \\
\hline & & LSEF & $\mathrm{PF}$ & $0.66 \pm 0.07$ & 0.86 \\
\hline & 3 & LESF & PF & $0.97 \pm 0.11$ & 0.84 \\
\hline 8 & & $\mathrm{PF}$ & $\mathrm{AF}$ & $1.33 \pm 0.17$ & 0.82 \\
\hline $\bar{T}$ & & LSEF & $\mathrm{PF}$ & $0.72 \pm 0.13$ & 0.67 \\
\hline 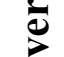 & $\mathfrak{\Xi}$ & LESF & $\mathrm{PF}$ & $0.97 \pm 0.20$ & 0.62 \\
\hline$\Xi$ & & $\mathrm{PF}$ & $\mathrm{AF}$ & $1.20 \pm 0.20$ & 0.75 \\
\hline$F$ & g & LSEF & $\mathrm{PF}$ & $0.57 \pm 0.08$ & 0.59 \\
\hline & t) & LESF & $\mathrm{PF}$ & $0.91 \pm 0.06$ & 0.87 \\
\hline & $z$ & $\mathrm{PF}$ & $\mathrm{AF}$ & $0.99 \pm 0.15$ & 0.65 \\
\hline
\end{tabular}

Figure 46. Table summarizing the slopes and determination coefficients $\left(R^{2}\right)$ extracted from the linear regressions of the velocities (analysis done in Figure 15). The methods' outcomes being $V_{\max }$ (Longitudinal CV) and $V_{\min }$ (Transversal CV) are initially separated into two categories. The methods are plotted against each other for a direct comparison. The substrates (WT and $m d x$ ) being two different entities were treated each aside first, then merged. $\mathrm{R}^{2}$ consistently scored higher values in merged sets (linear regressions in Figure 15 contain the merged data points) than in each set aside particularly for $V_{\max }$ values. The slopes of merged sets are closer to unity (in blue) except for the value in pink, where the slope is not close to one, but associated with a low $\mathrm{R}^{2}$ value as well. Values in red show sporadic unity slopes and in green slopes higher than one occurring when each of the WT and $m d x$ sets are treated separately. 
(This page is intentionally left blank) 



\section{Chapter 4}

\section{Discussion}

Microscopic components derived from active ionic and passive membrane properties, and macroscopic discontinuities reflecting branching anatomical features or other structural heterogeneities, can produce changes in the shape of the AP and CV, characteristics of cardiac conduction that cannot be predicted by continuous propagation models ${ }^{256}$. Discontinuous propagation implies that a delicate local source-sink balance governs successful conduction across the tissue, where the amount of charges supplied by the source proximally must at least be equal to the charges required to excite the cardiac membrane at the sink distally 257 . At the cellular level, this is controlled by membrane excitability followed by the state of electrical coupling between cells. In the ventricular myocardium, the principal active determinant of excitability is probably the cardiac

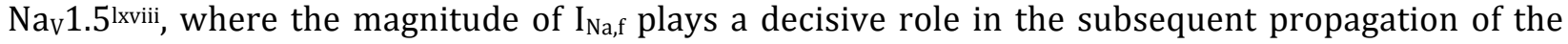
electrical wave from the source location further down the multicellular network. Meticulous opening and closing of the cardiac ion channels results in the generation of the AP, ensures its successful propagation, maintains the intricate coupling of electrical and mechanical activities and orchestrates the sequence of ionic channels to bring about the lucrative termination of the $\mathrm{AP}^{260}$. Henceforth, abnormalities of Nav1.5 expression, regulation or kinetics will translate into cardiac instabilities that induce electrical vulnerability and precipitate rhythm disturbances. How could $N a_{V} 1.5$ perturbations influence the stability of the entire cardiac tissue?

In this thesis work, two models of $\mathrm{Na}_{\mathrm{v}} 1.5$ abnormalities were investigated, and further modulation of $\mathrm{Na}_{v} 1.5$ was resumed through pharmacological interventions. The results of this thesis have tried to answer the questions proposed in the last section of the Introduction ${ }^{\text {lxix }}$ by:

1. Characterizing electrical instabilities in conduction in a model where $\mathrm{Na}_{v} 1.5$ is lost exclusively from the LM of the cardiomyocyte.

2. Implementing and validating different analytical strategies to evaluate conduction velocity in a medium with anisotropic and atypical spatial-temporal patterns of activation.

3. Investigating a circumstantiated proarrhythmic mechanism of Flecainide $\left(\mathrm{Na}_{v} 1.5\right.$ blocker) in normal heart tissues using clinically valid concentrations.

4. Providing adminicular evidence that a model harboring a LQTS3 mutation is exceedingly destabilized in the presence of Flecainide.

Ixviii Detailed description of the two models of propagation is found in the Introduction section 1.3.1.

lxix Refer to Introduction section 1.6 


\subsection{AP Conduction in the Murine Cardiac Tissue}

Some of the determinants of impulse propagation in the cardiac tissue include excitability of individual cells, impulse transmission between neighboring cells and structural geometry56, 551. Abnormalities in any of these components have been known in the general sense to facilitate reentrant arrhythmia for almost over a century578. By reducing membrane depolarization properties, such as excitability, CV is affected. The most important determinant of reduced excitability is the reduced availability of $\mathrm{Na}_{\mathrm{V}} 1.5$ leading to a reduction in $\mathrm{I}_{\mathrm{Na}, \mathrm{f}} \mathrm{f}^{368}$. Several pathological states could entail loss of functional $\mathrm{Na}_{v} 1.5$ such as acute ischemia ${ }^{579}$, 580, electrolyte disturbances ${ }^{581}$, genetic mutations ${ }^{390}$, remodeling processes ${ }^{582}$ and treatment with AADs ${ }^{315}$. However, our knowledge of how exactly each component contributes to reentry is still deficient, and our predictive potential of the trigger and sustenance of arrhythmia remains at best fairly unreliable.

It has been realized that the relationship between active (membrane excitability) and passive (cellcell coupling) components of cellular propagation and CV is far more complex, the characterization of which requires high resolution mapping techniques and computational models with the capacity to incorporate experimental data acquired at molecular, cellular and whole-organ levels ${ }^{246}$. In this regard, optical mapping has proven to be a fundamental experimental tool in the study of spatially extended electrophysiological heterogeneity of the cardiac substrate, without which appreciation of the complexity of arrhythmogenic models wouldn't have been possible. Whether slowing of conduction is a necessary condition or not for reentry, experimental and numerical data indicate that it's not sufficient. Additional factors (such as spatial-temporal heterogeneity for example) might be as crucial as conduction slowing to increase the propensity of the cardiac tissue for developing arrhythmic patterns.

\subsubsection{Impulse Conduction Slowing in the Dystrophin-Deficient Heart}

In a collaborative work, we showed through high spatial-temporal resolution mapping experiments suitable for the mouse heart that CV is significantly altered in the $m d x \mathrm{LV}$ free wall (Figure 10) 238 . As $\mathrm{Na}_{v} 1.5$ staining remains unchanged at the IDs in these hearts, reduction in $\mathrm{I}_{\mathrm{Na}, \mathrm{f}}$ as well as the decrease in CV have been attributed to the particular loss of $\mathrm{Na}_{\mathrm{V}} 1.5$ from the LM of the dystrophindeficient heart. The latter finding was sufficient to decrease Nav1.5 protein by $50 \%$ (Western blot), and total $\mathrm{I}_{\mathrm{Na}, \mathrm{f}}$ current by $29 \%$ (patch clamp) ${ }^{237}$. Macroscopically, these molecular findings were translated into CV decrease in both directions with $\mathrm{V}_{\text {max,mdx }}=0.55 \pm 0.08 \mathrm{~m} . \mathrm{s}^{-1}$ (decrease of 20-25\% compared to $\mathrm{V}_{\text {max,WT}}$ ) and $\mathrm{V}_{\text {min, } m d x}=0.31 \pm 0.03 \mathrm{~m}_{\mathrm{s}} \mathrm{s}^{-1}$ (decrease of $17-21 \%$ compared to $\mathrm{V}_{\text {min,WT}}$ ) (Figure 11a, Figure 22 Control) without a significant change in AR $=1.9 \pm 0.2$ (Figure 27a, Control). These results agree with a previous study reporting altered $\mathrm{Na}_{\mathrm{v}} 1.5$ expression and reduced excitability in the $m d x$ mouse ${ }^{583}$. Although the authors partly attributed these results to the reorganization of $\mathrm{Cx} 43$ (a major protein in GJ $\left.{ }^{1 \times x}\right)^{583}$, others and we couldn't confirm the presence of an ongoing Cx43 remodeling in the in $m d x$ heart ${ }^{238,584}$. Decreased Nav1.5 availability can be achieved experimentally with other conditions that increase the resting membrane potential (such as hyperkalemia581, anoxia $^{581}$ and acute ischemia ${ }^{580}$ ) or with $\mathrm{Na}_{v} 1.5$ blockers $^{315}$ (TTX ${ }^{\text {lxxi }}$ or AADs). Several studies reported an abrupt $\mathrm{CB}$ that occurred from relatively high enough velocities: from $\sim 0.25 \mathrm{~m} . \mathrm{s}^{-1}$ with AADs, $\sim 0.2-0.35 \mathrm{~m} . \mathrm{s}^{-1}$ with hyperkalemia protocols and $\sim 0.2-0.3 \mathrm{~m} . \mathrm{s}^{-1}$ in acute ischemia. Numerical studies, used to investigate conduction properties in cardiac tissues in the context of diminished excitability, revealed that reduced membrane excitability does not easily support ultimately slow

lxx GJ = Gap Junctions

lxxi TTX is introduced in section 1.4 
conductions $\left(\sim 0.17 \mathrm{~m} . \mathrm{s}^{-1}\right)^{\mathrm{lxxii}}$, rather the transition to CB is abrupt and occurs from a range of CVs that are relatively fast 585 . For extreme reduction in $\mathrm{Na}_{v} 1.5$ availability $(<11 \%)$, the generated depolarizing charge was reported to be no longer sufficient to depolarize the membrane to excitation threshold, so CB ensues ${ }^{585}$. Our values for the WT heart correspond well with the values reported in literature. In the direction of the long axis, the highest velocity values were recorded in the specialized tract of the ventricular conduction system ${ }^{586,587}$ with $V_{\max } \sim 2.1 \pm 0.4 \mathrm{~m} \mathrm{~s}^{-1}$, while the

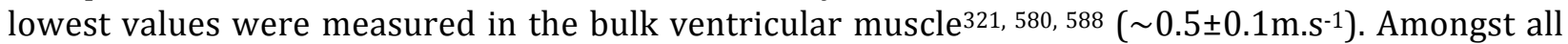
regions in the heart, where AR was calculated, the lowest is found in the ventricular muscle ${ }^{190}$ with $\mathrm{AR} \sim 2$; the highest being in the crista terminalis $(\sim 10)^{190,551}$.

Propagation velocities were measured simultaneously along the longitudinal and transversal axes of cardiac fibers in the LV free wall. The anisotropic distribution of propagation velocities shows that both velocities decreased by the same factor without causing a detectable change in AR in the $m d x$. Spach et al. assumed in their model of anisotropic propagation that the AR of velocities should not be altered when changes in membrane properties are considered uniform ${ }^{319}$. The loss of Nav1.5 exclusively at the LM is a clear non-uniformity in the membrane ionic properties. Hence this hypothesis cannot explain the unchanging AR in the $m d x$. On the other hand, a possible explanation can be provided by the discontinuous theory of conduction, which postulates that electrical anisotropy at the cellular level is affected by a sufficiently large number of factors, such as the shape and size of anisotropic cell, the amount and distribution pattern of GJ, and the anisotropic distribution of ionic channels 537 . As intracellular propagation is found not to be homogeneous in the cardiac cell and with the absence of change in intercellular coupling (GJ properties) in the $m d x$, the observed asymmetry in the distribution of $\mathrm{Na}_{V} 1.5$ in the $m d x$ cell should have resulted in a much larger percentage decrease in the transversal direction, causing a significant change in the AR.

Here, we hypothesize that the most likely explanation of this lack of effect is the smaller size murine cardiac cell lxxiii. In studies evaluating macroscopic conduction, little significance was given to the effects of size and geometry, before Spach et al. demonstrated that cellular scaling is as important as changes in other passive components (such as GJs) in determining the properties of conduction in the cardiac tissue ${ }^{589}$. Indeed, the smaller cell size and the relatively smaller cytoplasmic pathways are predicted to render intracellular dynamics less discontinuous ${ }^{590}$. The whole murine cell will be expected to depolarize as one unit, independently of the detailed distribution of its ionic channels, which would otherwise (i.e. in larger cardiac cells) alter subcellular electrical loading with significant effects on anisotropic conduction properties ${ }^{589}$. Consequently, it can be safely assumed that in conditions where CV is fast enough, the exclusive reduction of Nav1.5 from the LM in the $m d x$ cell causes a reduction of the overall depolarizing current entering the cell, decreasing CV monotonically independently of the axis of propagation and keeping the AR unchanged lxxiv.

\subsubsection{Possible Implications of LM Nav1.5 Loss in mdx: Contribution of LM to the Stability of Conduction}

Under physiological conditions, GJ conductance is not constant as excitation conduction takes place. The dynamical GJ model was first proposed by Vogel and Weingart (1998) ${ }^{258}$, who described a

\footnotetext{
Ixxii In contrast to the effects due to reduced excitability, Tsumoto et al. investigated the effects of intercellular coupling on AP conduction and confirmed through a mathematical study that even in the absence of GJ coupling, conduction can be maintained at velocities as slow as $0.14 \mathrm{~m} . \mathrm{s}^{-1}$ (Refer to section 4.1 .2 )

lxxii An adult canine cardiac cell has a length of $\sim 150 \mu \mathrm{m}$, whereas the length of a murine cardiomyocyte approximates to $70 \mu \mathrm{m}$. Canine neonatal cells are much smaller than canine adult cells $(\sim 30 \mu \mathrm{m})$ with distinct intercellular coupling.

lxxiv The lack of directional preferences on the properties of conduction between WT and $m d x$ was further challenged with Flecainide, where increasing the time scale of the upstroke and decreasing CV amplified the effects due to Nav1.5 asymmetry in the $m d x$ cell exposed to Nav1.5 blocking (section 4.3).
} 
mathematical model for the voltage- and time- dependency observed in junctional conductance changes measured in cell pairs 591,592 . A simulation study based on this model later investigated the effects of dynamical junctions on the shape and CV of the propagating AP593, where it was shown that GJ conductance increases moderately after the passage of the wave front. Therefore GJ can normally represent discrete resistive barriers for the flow of current, where increased resistance at these locations results in a discontinuous pattern of conduction at the cellular level244. Two mechanisms are currently proposed to explain AP propagation across GJs: the Gap-Junctional mechanism (GJM) and the Electric Field mechanism (EFM). The former states that modifying connexons' conductance (connexons are the structures forming the electrical connections between the cytoplasmic chambers of contiguous cells ${ }^{198,594,595}$ ) can have significant impacts on intercellular coupling 596, 597. Experimental findings ${ }^{598}$, reinforced with numerical studies ${ }^{599}$, have showed that reduced GJ coupling is indeed associated with CV slowing. However, the GJM failed to fully explain other experimental observations: Using genetically engineered cardiac-restricted $\mathrm{Cx} 43$ conditional KO mice, a decrease to $<10 \%$ of WT GJ conductance was associated with only a $50 \%$ decrease in $\mathrm{CV}^{600}$. Henceforth, it became more obvious that other mechanisms maintain AP propagation, when GJM is dramatically impaired.

The EFM was first suggested by Sperelakis et al. and postulates that during AP propagation, $\mathrm{I}_{\mathrm{Na}, \mathrm{f}}$ on the proximal side of the intercellular cleft of neighboring cardiac cells generates a large negative extracellular cleft potential, which in turn triggers the activation of the inward $\mathrm{I}_{\mathrm{Na} \text {, }}$ in the adjacent cells on the distal side of the cleft $601 . \mathrm{Na}_{\mathrm{V}} 1.5$ extensively clusters in the IDs (or the junctional membranes proximal and distal to the extracellular cleft) ${ }^{242,602}$, but is also expressed in the LM238, 603. In that regard, Tsumoto et al. elegantly investigated the role of subcellular $\mathrm{Na}_{v} 1.5$ distribution in AP conduction, where they elucidated the physiological significance of $\mathrm{Na}_{\mathrm{v}} 1.5$ in the LM in securing the stability of AP propagation, under conditions where cell-cell coupling is severely compromised ${ }^{604}$. They showed that Nav1.5 located in the IDs and LM play different roles in CV modulation. In normal intercellular coupling conditions, the effects of Nav1.5 in the LM are limited compared to the ones clustered at the IDs. However when conductance through GJ was severely diminished, the EFM became unstable with the absence of Nav1.5 from the LM and AP propagation was unsustainable. Contrastingly, the presence of Nav1.5 at the LM reinforced the EFM and compensated for the $\mathrm{CV}$ slowing and propagation remained successful in cases of dramatic loss of cell-cell coupling via GJs.

Our investigation of propagation in the in the $m d x$ mouse along with these studies could nicely fit into a clinical translation regarding the pathological progression of the cardiac phenotype in DMD. Cardiac involvement becomes clinically detectable only shortly before death with marked interstitial fibrosis and progression into dilated cardiomyopathy (DCM)605, 606, which precipitates heart failure 606,607 . At early stages of the disease, loss of Nav1.5 from the LM (although it causes a decrease in CV in all directions) doesn't compromise electrical activity spread in the presence of normal cell-cell coupling. As the disease progresses, intercellular coupling is severely reduced 608 due to extensive fibrosis. We speculate that as the propagation across the weakened junctions becomes unsustainable, the absence of $\mathrm{Na}_{\mathrm{v}} 1.5$ from the LM fails to provide homeostasis of AP propagation, therefore possibly facilitating conduction block and reentry, events known to occur in DMD patients at later stages of the disease.

\subsubsection{Methodological Approaches in Measuring CV}

Imaging techniques are widely used to investigate the spatial-temporal dynamics of biological systems, including excitable media such as the heart508, 510, 609. Optical mapping has become an increasingly important tool to study the electrophysiological properties of the heart. With its high 
spatial-temporal resolution, cardiac mapping has helped advancing knowledge of arrhythmias 610 and electrophysiological remodeling in diverse pathologies in the past two decades 292,611 . Principles and techniques of cardiac optical mapping have been extensively reviewed 505,514 . Multiple methods are used to process OAP and analyzing optical data, however no specific method has been reported to be optimal. Inappropriate processing of the data can lead to erroneous results and conclusions regarding the electrophysiology of the prep can be misleading 550 .

One advantage of optical mapping over other experimental electrophysiological techniques resides mainly in its ability to record electrical activity from more than 10,000 pixels simultaneously with a temporal resolution that exceeds $2 \mathrm{kHz}^{\mathrm{lxxv}}$. In our experiments, isochronal maps reveal that a point stimulation from a site near the center of the left ventricle (LV) creates anisotropic wave fronts, indicating that directional differences in conduction are characteristic of the cardiac tissue ${ }^{612}$. From epicardial measurements, AP generation and other electrophysiological properties of the cardiac substrate can be obtained via patterns of activation and the apparent CV in the medium of propagation. Direction and speed of propagation are used to characterize the electrophysiological properties of the cardiac substrate, from which CV is an important parameter to statistically describe the path of the electrical activity on the epicardium 532,613 .

CV depends on tissue properties and fiber orientation, the latter also depends on location 614 . Although epicardial CV can be quantified by different analytical strategies (Figure 11, Figure 12, Figure 13), caution was necessary while interpreting the results. Apparent epicardial CV is an accurate representative of the actual CV in the medium, only when wave fronts travel nearly parallel to the epicardial surface and to the field of view ${ }^{514}$. Therefore, we aimed at choosing constantly the activation interval (ROI) spanning a time window of $2.5-3 \mathrm{~ms}$ within the activation map (Figure $11 \mathrm{a}$, for example) excluding the first isochrone (in order to eliminate pacing artifacts) and last isochrones (affected by boundary conditions), therefore creating a region that essentially reflects the actual epicardial conduction (Figure 21a). Under quasi-physiological conditions (including $\mathrm{pH}$, temperature, $\mathrm{O}_{2}$ ), with mid-wall epicardial pacing of the WT LV $(10 \mathrm{~Hz})$, all measured velocities in the absence of Flecainide varied between $0.70-0.82 \mathrm{~m}_{\mathrm{s}} \mathrm{s}^{-1}$ along the longitudinal direction and 0.38$0.50 \mathrm{~m} . \mathrm{s}^{-1}$ transversally. Although velocity is a frequency-dependent parameter, these results are compatible with other optical mapping velocity measurements in the mouse heart using epicardial pacing with frequencies varying between $8-11 \mathrm{~Hz}^{284,615,616}$. In the sinus rhythm, activation occurred in a complex breakthrough pattern across the LV free wall. Since our optical mapping setup corresponds to projecting a 3D epicardium on a 2D surface, transmural components would complicate directional velocity estimates. Indeed, velocity maps were harder to compute due to the large area covered by the wave in relatively brief time intervals, causing the norms of the velocity vectors to blow up. These measurements were avoided in our analysis, and the reported velocities correspond to the paced rhythms that were initiated in the middle of the LV free wall in all preps.

The methodological study conducted after the initial finding in the $m d x$ mouse aimed at investigating the working range of each of the analytical strategies implemented in measuring CV in the mouse heart using optical mapping. Interestingly, our experimental data reveal that all methods yielded consistently the initially observed findings (Figure 11, Figure 12, Figure 13), confirming a similarity to the results we published for the $m d x$ mice, where the Least Square Ellipsis Fitting (LSEF) method was implemented Figure 10) ${ }^{238}$. Exposure of the $\triangle \mathrm{KPQ}$ heart to Flecainide (Figure $14 \mathrm{~b}$ ) caused the activation to deviate from the expected anisotropic pattern. Regardless of the underlying molecular mechanism, complex patterns in the cardiac substrate aren't always

Ixxv The recording chip of the CMOS camera contains 100 by 100 pixels offering a spatial resolution of $100 \mu$ m. The frame rate was set to $2 \mathrm{kHz}$ when measuring with Di-4-Anepps. Refer to Experimental Methods (section 2.1.4) for further details. 
avoidable. In such cases, activation patterns aren't comparable, because the heterogeneity effect cannot be predicted from the initial conditions imposed by pacing. The CV values will be expected to deviate not in between analytical strategies only, but also on a prep-by-prep basis. That said, care should be taken while interpreting these results, due to the loss of predictability and to the fact that the methods are conceptually different. The condensation of early isochrones seen in the $\triangle \mathrm{KPQ}$ hearts with Flecainide, is probably due to a severe epicardial conduction decrease around the pacing electrode. Cardiac layers are reported to react differently to $\mathrm{Na} v 1.5$-blocking agents ${ }^{492,499,503 .}$ Hence, we speculate that the isochrones become largely spaced, due to a stronger 3D intramural component at the pacing site, which lead to the reappearance of the epicardial wave near the boundaries at later time points, an effect, which simulates an increase of the apparent mean CV, when the electrical excitation is mapped on a 2D plane. No such effect was seen in the WT measurements at $5 \mathrm{~min}$, treated with the same protocol as the $\triangle \mathrm{KPQ}$. Gradual decrease in $\mathrm{CV}$, without alteration in the elliptical behavior was the only detected effect with WT at similar measurement times in terms of isochronal maps.

The fact that all methods performed well with homogeneous elliptical propagation cannot be extrapolated in a straightforward manner to include success of these methods with more complicated patterns. Whether the complexity is due to the substrate heterogeneity (Figure 17) or noise embedded in the signal (Figure 16), these factors can complicate the analysis of conduction in murine hearts. Measurement noise can replicate non-homogeneous activations, due to misdetection of the appropriate activation times, a complication primarily due to technical errors rather than the nature of the cardiac substrate itself. On the other hand, dissimilarities, induced by manipulating the diffusion properties of the medium at the level of each pixel, impose an increasing level of heterogeneities in the medium, a proposed mechanism in the initiation of arrhythmia ${ }^{502}$. This is considered a characteristic of the investigated substrate at hand. We showed how these factors could significantly alter CV depending on the method of analysis.

Effect of Measurement Noise: Area Fitting (AF) is the most robust. Activation maps were completely disrupted as the SNR deteriorated (Figure 16). Although the algorithm is automated to detect precisely activation times, as in the case of the experimental data, detection became increasingly ambiguous for the surrogate noisy data (Figure 16a, upper row). The LSEF depends on the ability to fit ellipses to data points. When isochrones became globally ill defined for any reason, fitting ellipses become a random process (Figure 16a, middle row). However even at lower pixel SNR a linear regime could still be sporadically found for some maps, where the linear fitting method could give considerably different regression lines, leading to arbitrarily velocity values. Using the Plane Fitting (PF) method, the ability to compute a gradient for any field stands at low SNR (Figure 16a, third row). Nevertheless, it's difficult to judge the validity of these results since the vector field isn't reliable, with random amplitude vectors pointing in random directions. AF, in contrast to the previous two methods, overcomes misdetection of activation times. The extreme noise level didn't hinder the potential for detecting the area that activated and quantifying the activation speed. The erroneous detection of APs when there's none-due to an ill-defined absolute zero, i.e. the arbitrary time point between 2 pulses-lead to few activated pixels per image in the earlier frames (Figure $16 \mathrm{~b}$, times up to $5.5 \mathrm{~ms}$ ). However, when the real activation eventually dominated, the time series increased accordingly (as presented for the experimental data in Figure 13a and b, right panels). This method doesn't have an inherent problem and is spatially independent. The higher the noise, the higher the number of pixels that could be erroneously detected as activated, rendering the absolute zero time of actual activation harder to specify. Nevertheless, with the appropriate correction of the initial time $\left(\mathrm{t}_{0}\right)$ the method ably detected the real activation (Figure 16b) and quadratic functions were reliably fitted to the time series. Hence, plausible value for anisotropy could still be quantified. In summary, the inability to have a well-defined activation map at 
extremely low SNR (SNR<0.01) eliminated the plausible use of the LSEF and PF in these conditions. $\mathrm{AF}$ can falsely detect the earliest pixel due to noise. However, carrying these corrections allows AF to be the method of choice in evaluating CV in the presence of very low SNR without contributing significant changes to the activation other than the physiological process of interest.

PF Quantifies Regional Differences in Propagation. In simple experimental conditions, while measuring the epicardial activation, the assumption that the epicardium could be treated as a homogeneous and continuous medium fairly stands ${ }^{613}$. Investigating disease models or pharmacological interventions could impose variable activation configurations, which by significantly deviating from the expected elliptical spread, necessitate statistical calculations embedded within the returned values of velocities. PF displays velocity estimates as vectors that locally characterize the speed and direction of propagation qualitatively and quantitatively during simple patterns or activations, even where anisotropy is completely lost. It offers an efficient, quantitative strategy in constructing vector fields that fully make use of the local inter-isochronal information ${ }^{284}$, a possibility that isn't offered by the LSEF, nor the AF. These latter methods describe the conduction in the medium only globally. Despite its independence of geometry, the outcome of AF can be used only as an "effective" velocity measure, a rate of wave propagation in many different orientations, rather than an "actual" velocity. LSEF shows to be insensitive to local changes, making it a poorer choice as the level of asymmetry adds up (Figure 17a, middle row). The LSEF (and AF to a lesser extent) fails as well to adequately represent the complexities of the activation pattern in the final results. The effect of heterogeneity is well illustrated with PF (as seen in polar plots, comparing Figure $14 \mathrm{a}$ and $\mathrm{b}$ ) that captured these important fluctuations in the medium and incorporated them as a statistical component within each bin, by progressively increasing the SD, a value associated by default with the velocity value (Figure 17b, right panel). However, this latter could also be regarded as a disadvantage: Based on numerical derivatives, overestimating mean $\mathrm{CV}$ is a risk and increasing SD means loss of precision, because knowing the "real" velocity in the medium is simply not available. Henceforth, caution in interpreting results is necessary. This method is independent of geometry, so regardless of the underlying fiber orientation; the maximal and minimal velocities are picked up from binning local vectors.

LSEF is a Poorer Choice as the Degree of Asymmetry Increases. The use of geometric models allows for a fast and robust assessment against outliers in data points. The cost function reasonably characterizes the goodness of fit of the fitted ellipses to the different isochrones, hence objectively reports deviations of the activation contours from the expected anisotropy. Such a model takes into account the anisotropic spread in the heart and shows robustness in averaging out small-scale nonuniformities in the morphology of the propagating wave front that may otherwise cause divergence. On the other hand, the same intrinsic properties make this method particularly a poorer choice, as the anisotropy in the cardiac tissue is disrupted. First, only the isochrones that show an elliptical propagation from a point stimulation are valid for analysis (Figure 10a, Figure 11a and b). This method assumes symmetry about the pacing point, making it inappropriate for any asymmetric configuration, as seen in the effect of $\mathrm{Na}_{\mathrm{V}} 1.5$-blocking (Figure 14b) and confirmed with synthetic data (Figure 17a). Second, CV evaluation is limited to isochronal contours, with no use of the information lying between the linearly interpolated pixels. In retrospect, the isochronal contours depend on the ability to detect activation times that are by themselves dependent on noise (measurement noise, motion artifacts) and sampling rate. Third, the choice of particular isochronal points depends on the conserved symmetry and the value returned by the cost function. A threshold is therefore necessary to either include a particular isochrones or exclude it. In consequence, isochrones showing asymmetry of the contours due to the ellipsoid shape of the heart or to boundary conditions are excluded, which limits the ROI to fewer than 5-6 isochrones (Figure 11a and b, left panels). Fourth, our data showed that deviations from anisotropy cause each fitted 
ellipsis to assume a separate center that could drift significantly from the pacing site, which is the natural point of activation for all contours. This center drift affects the lengths of the axes and causes scattering in isochronal points, rendering a linear fit invalid for velocity evaluation.

Limitations. Our study is applicable to 2D isochronal maps. The ellipsoid shape of the heart alters the detection of activation times near the boundaries. Adopting a ROI approach, where the wave can be safely assumed to travel parallel to the epicardial surface, considerably reduces this effect. Modifications are necessary if those methods are to be applied on a 3D model. Another limitation is the inability to correlate the outcomes of each method with epicardial fibers' orientation. To further strengthen the arguments presented, a direct correlation between fibers' orientation and velocities should be emphasized.

\subsection{Reduced Excitability and APD Prolongation in mdx-hearts}

The cardiac tissue is inherently anisotropic, a property, which has been implicated in arrhythmogenesis 520,617 . In our experiments, the $m d x$ mouse provides a heart model of lower excitability (due to loss of Nav1.5 exclusively on the LM) compared to its WT counterpart. We additionally chose to alter excitability in these cardiac substrate by using Flecainide, which blocks $\mathrm{Na}_{\mathrm{v}} 1.5$ in its open state ${ }^{511,618}$ (as described in the Introduction section 1.4.7, the availability of the binding site of the drug is channel-state dependent ${ }^{619}$ ). The slow binding and unbinding kinetics of Flecainide are at the core of its use-dependence effects; a property of incremental blocking with successive beats 454,619 . We have estimated in our experiments the number of successive beats (2030 beats in a train of 60 pulses) necessary from the start of pacing until steady state blockade was reached at each time point, during exposure to Flecainide when a measurement is recorded. It should be noted here, that the term "steady state" doesn't refer to a true equilibrium of drug blockade, rather to the sequence of repetitive APs of similar morphology that indicates steady state conditions. It's expected that fluctuations of drug blockade take place during each cycle of pacing as $\mathrm{Na}_{v} 1.5$ shifts between the different conformational states ${ }^{118}$. For open-state blockers, the percentage of drug-bound sites increases very fast initially then starts to decrease, as the binding site remains open ${ }^{459}$. This process occurs during the brief time interval of depolarization, $(\sim 1 \mathrm{~ms})$ during which $\mathrm{Na}_{v} 1.5$ opens, followed almost instantaneously by fast inactivation 11,620 .

By lowering $\mathrm{I}_{\mathrm{Na}, \mathrm{f}}$ using Flecainide in the WT and maybe furthermore in the $m d x$, we studied how various factors might come into play in facilitating preferential longitudinal or transversal block in anisotropic preparations where the whole hearts remain intact. Preferential directional block has been investigated previously and the interventions that decrease Nav1.5 availability in the anisotropic tissue have been leading to conflicting results. Spach et al. showed that anisotropy could lead to a preferential decrease in the longitudinal direction, facilitating conduction block and reentry ${ }^{319}$. They also argue that despite larger discontinuities along the transversal direction, propagation was more robust and maintained primarily by the continuously larger maximal upstroke velocities measured in this direction ${ }^{319}$. In Spach's model of directional differences in open-state blocking, the author argue that since the rate of rise of the AP was measured to be faster along the transversal direction in their preparations, the opening time of Nav1.5 must be shorter during depolarization along this direction, than along the longitudinal one ${ }^{621}$. They also argue that drug molecules are expected to behave in a non-biased fashion, i.e. irrespective of anisotropic considerations, whenever the channels' binding sites suddenly become available the drug molecule should bind, following the principle of mass action of a random distribution ${ }^{622}$. Henceforth, small directional differences (occurring on lower time scales than a $m s$ ) in the opening time of the channel would result in considerable differences in the blocking mechanism of Nav1.5 open-state antagonists 621. 
Other investigators reported that transversal block was more favorable in the anisotropic tissue ${ }^{623-}$ 625 , particularly when GJs conductance was severely reduced using heptanollxxvi624. Another study by de Diego et al. showed that under conditions where $\mathrm{I}_{\mathrm{Na}, \mathrm{f}}$ was reduced in anisotropic tissues, whenever $V_{\max }$ and $V_{\text {min }}$ decreased proportionately keeping the AR unaltered, conduction block was not observed626. Indeed similar changes in $\mathrm{V}_{\max }$ and $\mathrm{V}_{\min }$ were observed in the $m d x$ heart, keeping the AR unaltered under no treatment conditions (Figure 27). As the velocity in the medium increased with Flecainide at later stages of the protocol (Figure 22), AR was significantly altered (Figure 27).

\subsubsection{Relationship between Nav1.5 Availability, (dF/dt) $\max$ and CV}

In the $m d x$ mouse, $\mathrm{I}_{\mathrm{Na}, \mathrm{f}}$ is decreased by $\sim 29 \%$ (Patch clamp) ${ }^{237}$, which translates into a global CV decrease ${ }^{\mathrm{lxxvii}}$ (along both axes of propagation) ${ }^{238}$. In light of these findings and the fact that the resting membrane potential in the $m d x$ cells is not more depolarized than the one in the WT237, we measured the upstroke velocities of the propagating wave on the epicardial surface in both directions and we found that $(\mathrm{dF} / \mathrm{dt})_{\max }$ correlates well with CV measurements on two levels: First, $(\mathrm{dF} / \mathrm{dt})_{\max }$ is larger along the long axis and smaller transversally in both WT and $m d x$ (i.e. directional differences exist at the level of the upstroke velocities). Second, (dF/dt) $\max$ acquires lower values in the $m d x$ than in the WT. Interestingly, our calculations show that $(\mathrm{dF} / \mathrm{dt})_{\max }$ decrease in the $m d x$ is proportional to the decrease in $\mathrm{I}_{\mathrm{Na}, \mathrm{f}}$ found in patch clamp experiments, with a $\sim 28 \%$ and $\sim 30 \%$ decrease in the $m d x$ with respect to the WT along the longitudinal and transversal directions respectively (Figure 24a and b, Control conditions). The percentage decrease in the velocities measured in the $m d x$ was lower than the ones observed in $(\mathrm{dF} / \mathrm{dt})_{\max }$ and $\mathrm{I}_{\mathrm{Na} \text {,f }}$ with $20-25 \%$ in $\mathrm{V}_{\max }$ and $17-21 \%$ in $V_{\min }$ (Figure 22a and $b$, Control conditions). These findings suggest that propagation in the murine epicardium exhibits both continuous and discontinuous properties ${ }^{\mathrm{lxxviii}}$. The proportionality relationship between the decrease in $\mathrm{I}_{\mathrm{Na}, \mathrm{f}}$ and $(\mathrm{dF} / \mathrm{dt})_{\max }$ in either direction in the $m d x$ is a property of continuous propagation ${ }^{92,627}$. However, $(\mathrm{dF} / \mathrm{dt})_{\max }$ also shows dependence on the direction of propagation relative to the axis of the cardiac cells, which is a property of rather discontinuous propagation ${ }^{319}$. In a continuous medium, the effect of axial resistance (presence of GJ along the longitudinal axis of the cell and its absence laterally) is to simply shrink the spatial scale over which depolarization takes place, leaving the time course of depolarization practically unchanged ${ }^{251}$. In other words in a continuous medium, $(\mathrm{dF} / \mathrm{dt})_{\max }$ is expected to reflect excitability of the epicardium independent of the direction in which the wave propagates. As explained in section4.1.1, the size of the cardiac cell plays a major role in propagation. It's suggested that the murine epicardium can be lumped into a "single macroscopic model cell" 254 , whenever the smaller size murine cardiac cells are present in a homogeneous tissue 551 , with a regular network of electrically well-coupled cells ${ }^{628}$. Despite the fact that in the murine heart intracellular dynamics can be assumed to be almost continuous ${ }^{590}$, the directional changes in the measured $(\mathrm{dF} / \mathrm{dt})_{\max }$ show that cardiac propagation in the murine epicardium evidently acquires discontinuous properties.

Although it has been shown that neither the relationship between $I_{N a, f}$ and $(\mathrm{dF} / \mathrm{dt})_{\max }$, nor the relationship between $(\mathrm{dF} / \mathrm{dt})_{\max }$ and $\mathrm{CV}$ are linear in systems with discontinuous propagation, it has been assumed that effects of AADs on CV should correlate directly with their effects on $(\mathrm{dF} / \mathrm{dt})_{\max }$. We show CV (Figure 21a) and (dF/dt) $\max$ dispersion maps (Figure 23a and b) within a ROI on the

\footnotetext{
lxxvi Heptanol is a chemical substance used to modulate the junctional current promoting uncoupling by decreasing the opening probability of GJ channels. Reference: American Journal of Physiology (1992) 262:C1531-8.

lxxvii Refer to section 4.1.1.

lxxviii Refer to the Introduction section 1.3 .1 for a detailed description of the differences between continuous and discontinuous conduction in the cardiac tissue.
} 
epicardial surface for WT and $m d x$ under conditions, where no treatment was applied and at $t_{10}$ from exposure to Flecainide. It became evident that both hearts initially respond to Flecainide with a decrease in $(\mathrm{dF} / \mathrm{dt})_{\max }$ and $\mathrm{CV}$ in almost all locations, in both substrates. WT hearts however showed a peculiarity in their conduction profile: a focus of increased velocities mainly along the long axis of the cells became evident at $t_{10}\left(\sim 60 \%\right.$ increase in $V_{\max }$ in this example, Figure $21 \mathrm{a}$, lower row, left). In Figure 22a, box plots started to show a collective increase in $V_{\text {max }}$ within the WT group at $7 \mathrm{~min}$ from exposure to Flecainide. In the meantime, the $m d x$ heart remains devoid of any heterogeneity in conduction (Figure 21a, lower row, right) and CV was continuously decreasing (Figure 22a and b). From (dF/dt) max maps (Figure 23a and b) and bar graphs (Figure 24a and b), the maximum AP upstroke velocity was responding to Flecainide by a continuous decrease in either substrate. The peculiarity observed in the CV profile of the WT at $t_{10}$ was not correlated with an increase in $(\mathrm{dF} / \mathrm{dt})_{\max }$ at the same location. This indicates that $(\mathrm{dF} / \mathrm{dt})_{\max }$ and $\mathrm{CV}$ varied in the same direction up to $7 \mathrm{~min}$ exposure to Flecainide, beyond which they changed in opposite directions.

Examples of such "supernormal conduction" were previously reported in the canine 99,549 and sheep ${ }^{97}$ Purkinje fibers, where no uniform relationship between $(\mathrm{dF} / \mathrm{dt})_{\max }$ and CV was demonstrated at different experimental conditions including hyperkalemia, hypercalcemia and with the use of drugs (such as phenytoin, digoxin). Dominguez and Fozzard ${ }^{97}$ and others ${ }^{549}$ found no alteration in cable properties sufficient to explain speeding of conduction in sheep Purkinje fibers subjected to hyperkalemia, in the absence of a more depolarized resting membrane potential or shift in the threshold potential. They suggested therefore that the likely explanation for the speeding of conduction was increased "excitability" that developed as the takeoff potential was reaching threshold. Excitability of the cardiac tissue was described as a confluence of conditions such as the relative proximity of the membrane potential to threshold during terminal repolarization, slow diastolic repolarization and alterations of the ionic environment, which could be experimentally measured by the amount of injected current necessary to bring the tissue to threshold ${ }^{99}$.

Our experimental results show that as WT hearts were exposed to Flecainide, CV was considerably increased (the value reached was $\sim 60 \%$ larger at $t_{10}$ than it was originally at $t_{0}$ ) in finite regions of the heart under steady pacing conditions with no evidence of a simultaneous increase in $(\mathrm{dF} / \mathrm{dt})_{\max }$, nor excitability. Instead, speeding of conduction occurred in regions of spatially extended decrease in upstroke velocity. Kléber and Rudy suggested a transient decrease in $\mathrm{I}_{\mathrm{Na}, \mathrm{f}}$ threshold requirements to account for supernormal conduction ${ }^{254}$. As the wave emanates from the stimulation site, depolarizing charges bring the depressed (lower available Nav1.5 for excitation) and quiescent tissue to threshold. Simply put, an area of transient decrease in $\mathrm{I}_{\mathrm{Na}}$, activation would require less depolarizing charges for Nav1.5 activation. In the presence of decreased upstroke velocity, the rate of change of membrane potential at takeoff potential is also reduced and slow depolarization to peak voltage will increase the electrochemical driving force for $\mathrm{Na}^{+}$, hence could cause a transient increase in $\mathrm{I}_{\mathrm{Na}, \mathrm{f}}$ and $\mathrm{CV}$ in that finite region of the heart ${ }^{629}$.

This mechanism, although speculative, may account for the local increase in CV in the presence of depressed excitability, but doesn't explain why the $m d x$ heart, with lower amount of $\mathrm{Na}_{\mathrm{V}} 1.5$, failed to develop the same speeding of conduction under the same experimental conditions. Nonetheless, we have shown using optical mapping and by lowering $\mathrm{Na}_{v} 1.5$ availability using Flecainide, that longitudinal velocity is enhanced whereas transversal velocity is reduced under steady state pacing, favoring therefore the probability of a transversal block occurring in the setting of a premature beat. A limitation of optical mapping, as explained in the previous section of this chapter (4.1.3), is that the increase or decrease in CV of the electrical wave propagating on the epicardial surface does not necessarily reflect the dynamics of the electrical activity in unmapped subepicardial layers. A transmural study is consequently necessary to investigate subsurface events with Flecainide. 
We extensively showed in section 3.1, that the non-uniform loss of $\mathrm{Na}_{\mathrm{v}} 1.5$ from the LM of $m d x$ cardiomyocytes lead to a global decrease in CV in all directions, keeping the AR unaltered. In the presence of directional differences in the measured (dF/dt) $\max$ (Figure 23 and Figure 24), we recognize that the mouse epicardium exhibits a degree of discontinuous propagation, despite the smaller size of murine cardiomyocytes. Under conditions of Nav1.5 blocking, $(\mathrm{dF} / \mathrm{dt})_{\max }$ overall decrease between $t_{0}$ and $t_{10}$ (Figure 27c) was on average similar in both directions in the WT (a decrease of $51 \pm 17 \%$ along the longitudinal axis and $52 \pm 10 \%$ transversally), whereas the $m d x$ heart showed slightly different directional changes in $(\mathrm{dF} / \mathrm{dt})_{\max }$ with Flecainide (a decrease of $44 \pm 9 \%$ longitudinally, $39 \pm 6 \%$ transversally). Despite these similarities in the overall decrease of $(\mathrm{dF} / \mathrm{dt})_{\max }$ longitudinally and transversally in the WT heart (Figure 27c), the decay curves (Figure 25) highlight a directional discordance in the decay rates of this parameter notably in the early 5 minutes of exposure to Flecainide. This suggests that the time course of the upstroke not only is dependent on the spatial configuration in the normal heart, but its modulation by Flecainide is also subject to anisotropic considerations. This indicates a potential stronger involvement of discontinuities in propagation of the murine epicardial AP with Nav1.5 blocking.

We have argued in section 4.1.1 that in contrast to the inhomogeneous intracellular propagation expected in large cardiac cells ${ }^{537}$, murine cells could behave as a syncytium due to their relatively smaller size and their presence in a network of strongly well-coupled units, that intracellular inhomogeneity is of minor influence on macroscopic anisotropic conduction ${ }^{539}$. To further refine this argument, the latter statement stands for small size cells particularly when CV in the medium is fast enough (as discussed in section 4.1.1, where these conditions were fulfilled). Cellular scaling could be dynamically described by intracellular conduction, i.e. by how fast the depolarizing charges that enter the quiescent cell from one end propagate within it, can progressively change the membrane potential and trigger $\mathrm{Na}_{v} 1.5$ activation. By blocking $\mathrm{Na}_{\mathrm{v}} 1.5$ and reducing the velocity of the traveling wave in the medium, local depolarization dynamics also become slower. On that scale, the small size cell becomes larger; therefore displays large size cells intracellular dynamics.

While this accounts for the directional discrepancy observed in the rate of decay of $(\mathrm{dF} / \mathrm{dt})_{\max }$ in the WT heart, the rate of change in $(\mathrm{dF} / \mathrm{dt})_{\max }$ in the $m d x$ becomes more understandable (Figure 25a and $b$ ) by considering the following: the longitudinal $(\mathrm{dF} / \mathrm{dt})_{\max }$ decay curves for the WT and $m d x$ are almost superimposable (Figure 25a, left), the transversal ones significantly diverge from one another in favor of the WT curve, which decays at a much faster rate in the early phases of the treatment (Figure 25a, right). The decay curves along the longitudinal direction reveal almost similar kinetics between the $m d x$ and WT, suggesting that clustering of Nav1.5 at the GJs and the passive properties at the IDs are not altered between the two substrates with the addition of Flecainide. With the lack of Nav1.5 on the LM of cardiomyocytes in the $m d x$ heart, modulation by Flecainide along the transversal direction is no longer possible. A sequel of Flecainide's activity on the available channels at the IDs could account for the observed decay in $(\mathrm{dF} / \mathrm{dt})_{\max }$ in the $m d x$ heart along the transversal direction.

It's particularly important to mention that the latter interpretation is valid with the assumption that the epicardial layer, where activation is measured, is a perfect 2D plane of cells (from a geometric perspective) and transmural effects on activation are assumed to be negligible. This is a limitation of the experimental method, whenever a 3D object is projected on a 2D surface. In reality, the mapped epicardial layer is not isolated (in the intact heart) and transmural components could influence CV measurements, if the wave traveling across the wall is for some reason faster than the epicardial propagation. This could account for an "apparent" increase in velocity or "supernormal conduction" in the presence of depressed excitability. 


\subsubsection{AP Early Phase Prolongation: A Localized Effect of Nav1.5 Loss}

The repercussions of the considerable reduction (29\%) in $\mathrm{I}_{\mathrm{Na}, \mathrm{f}}$ in the $m d x$ cells ${ }^{237}$ are not limited to $(\mathrm{dF} / \mathrm{dt})_{\max }$ (Figure 24, Control), nor CV (Figure 22, Control) in the dystrophic substrate, but they were also inflicted in APD prolongation, particularly $\mathrm{APD}_{25}$ (Figure 32a, Control) with an increase of $\sim 60 \%$ in $\mathrm{APD}_{25}$ along either direction. The effects of Nav1.5 loss in the $m d x$ become increasingly less significant beyond the early stages of repolarization; hence almost no difference in APD is detected at $50 \%$ repolarization (Figure $30 \mathrm{a}$ and b) or beyond (Figure $32 \mathrm{~b}$ ). This result emphasizes the specificity of ionic channels' involvement with in the context of dystrophin deficiency. Nav1.5 loss under control conditions in the $m d x$ heart orchestrates a temporally localized effect during the sequential activation of ionic channels that follows phase 0 of the AP. This is tailed by a fast enough repolarization that eventually compensates for the previous delay in phase 1 and early phase 2 to bring the $\mathrm{AP}_{m d x}$ to phase 4, at the same time as the $\mathrm{AP}_{\mathrm{WT}}$ (Figure 19). The direct influence of $(\mathrm{dF} / \mathrm{dt})_{\max }$ on $\mathrm{APD}_{25}$ was further elucidated in Figure 35. Although an exact quantification of this relationship cannot be computed from this graph, it was apparent that fast enough upstrokes (i.e. $\mathrm{WT}_{\text {Long) }}$ ) were correlated with brief $\mathrm{APD}_{25}$ (Figure 35a) and as the upstroke velocities became continuously smaller (whether for $\mathrm{WT}_{\text {Trans }}$ or $m d x$ either direction), $\mathrm{APD}_{25}$ became more prolonged. Since the time scales of the processes that follow the upstroke are of finite value, an increasingly faster $(\mathrm{dF} / \mathrm{dt})_{\max }$ will not reduce $\mathrm{APD}_{25}$ beyond the minimal time required for $\mathrm{I}_{\mathrm{to}, 1}$ to activate and inactivate. On the other hand, interventions that reduce the upstroke velocity could alter $\mathrm{I}_{\mathrm{to}, 1}$ kinetics and amplitude significantly, due to the latter's strong outward rectification properties with a dependency on the change in $V_{m b r}$ as phase 0 reaches its peak ${ }^{107,630}$. In conditions where no Flecainide is added, $\mathrm{I}_{\mathrm{Ca}, \mathrm{L}}$ kinetics of activation are considered relatively slow to contribute substantially to the upstroke or the early phases (25\% repolarization) that follow the upstroke ${ }^{162}$. Therefrom, we could attribute the less prominent notch, decreased phase 1 and higher plateau in $\mathrm{AP}_{m d x}$ with a restricted APD prolongation to the consequential loss of $\mathrm{Nav} 1.5$ that generated a shallower upstroke and delays in early repolarization in the $m d x$ heart.

The effects observed in $\mathrm{APD}_{25}$ are not limited to the epicardial locations, from which the APs were picked, but they exceed these local values to the spatial distribution of each of the AP repolarization levels. APD dispersions ( $\triangle$ APD) were extracted from the corresponding APD maps (Figure 28 and Figure 31 ) to quantify the extent of APD repolarization differences in the mapped epicardial surfaces. Although histograms (Figure 29) do not provide information regarding the spatial distribution of the heterogeneities whenever found, we could visualize the APD spatial configuration directly from the maps themselves. For the first time, our data show that Nav1.5 loss from the LM in the $m d x$ not only associates with prolongation of $\mathrm{APD}_{25}$, but also contributes significantly to the widening of the $\triangle \mathrm{APD}_{25}$ by more than $60 \%$ compared to its WT counterpart (Figure 33). As the AP approaches full repolarization, the effects due to lower Nav1.5 availability become weaker and consistently no significant change in the dispersion of APD values is observed for either $\mathrm{APD}_{50}$ or $\mathrm{APD}_{75}$ (Figure 33). This indicates that despite $\mathrm{Na}_{\mathrm{V}} 1.5$ intense clustering at the IDs 242,244 in the normal heart, the remaining $\mathrm{Na}_{v} 1.5$ channels on the LM obviously play a crucial role in homogenizing APD values and their distribution at early phases of APD repolarization, for their loss has the potential to significantly influence the extent of spatial heterogeneity up to more than $60 \%$. Since this widening of dispersion is restricted to $\mathrm{APD}_{25}$, and doesn't include $\mathrm{APD}_{50}$ or $\mathrm{APD}_{75}$, the direct implication of such a finding in arrhythmogenesis is yet to be clarified. This might also point to the possibility that the $m d x$ cell could have developed a compensatory repolarization machinery that smoothes out later repolarization abnormalities in APDs in value and dispersion at the level of the multicellular tissue. 


\subsection{A Novel Mechanism Behind Flecainide Proarrhythmia}

The experimental findings, which appear closer to our observations with Flecainide, were published by Krishnan and Antzelevitch in 1993, in which high concentrations of Flecainide ([10-20 $\mu \mathrm{M}]$ ) were capable of not only inducing differential responses between endocardium and epicardium in the dog heart, but rather opposite effects in canine epicardial strips with either prolongation or abbreviation of the APD at different exposure times to the drug411. Canine epicardial and endocardial preparations were perfused with Flecainide and were paced at different cycle lengths (CL $=200-2000 \mathrm{~ms}$ ). Transmembrane potentials recorded from one or a few more sites on the epicardium using glass microelectrodes, measuring upstroke velocities, $\mathrm{APD}_{50}$ and $\mathrm{APD}_{90}$. They observed that Flecainide $[15 \mu \mathrm{M}]$ produced prominent prolongation of both $\mathrm{APD}_{50}$ and $\mathrm{APD}_{90}$ in the epicardium after $30 \mathrm{~min}$ of Flecainide exposure. Along with the increase in APD, was an increase in the delay between the upstroke of phase 0 and phase 2 of the AP, and diminution of the upstroke velocities. However, after $40 \mathrm{~min}$ exposure to Flecainide, further decrease in phase 1 resulted in an "all-or-none repolarization" in the epicardial cells, leading to loss of the plateau and a premature termination of the AP411. These experiments clearly show that Nav1.5 blockade can produce opposite effects on the APD in the epicardium. As they recorded from three sites on the epicardium, it was observed that APD prolongation or abbreviation was rate and time dependent, where different sites displayed distinctive rate dependent properties. APD restitution curves measured from the three sites were almost congruent at shorter S1-S2 intervals $(\sim 150-300 \mathrm{~ms})$ with a basic cycle length of $2000 \mathrm{~ms}$, and diverged considerably at S1-S2 intervals larger than $350 \mathrm{~ms}$ up to $600 \mathrm{~ms}$.

Despite some similarities between the latter observations and ours, particularly with epicardial APs that initially showed a prolongation with Flecainide followed by an abbreviation, major and crucial differences exist between their results and ours on several scales. The comparison between their results and ours should be done with caution: starting from the different animal models used (canine vs. murine hearts), the experimental tools to characterize the electrophysiology in the tissue (extracellular electrodes at a few sites on the epicardium vs. optical mapping using 10,000 recording sites and a spatial resolution of $100 \mu \mathrm{m}$ ), the high concentrations of Flecainide that are of little practical significance in a clinical setting631, $632(15 \mu \mathrm{M}$ vs. $1 \mu \mathrm{M}$; therapeutic range of Flecainide $[0.5-2 \mu \mathrm{M}]^{1 \mathrm{xxix}}$ ) and the adopted pacing protocol to reveal the heterogeneity in the epicardium (S1-S2 protocol vs. steady state pacing at a fixed BCL). The impacts of these differences are tackled in a more detailed manner in the next subsections (4.3.1 and 4.3.2).

The concentrations of drugs Krishnan and Antzelevitch used in their in-vitro studies were very high compared to ours ${ }^{411}$. The trend in pharmacological interventions is to use as high concentrations as possible, until an effect is observed ${ }^{633}$, 634 . Although these pharmacological studies are of great scientific importance, serious questions should be raised concerning the ones that use tens or hundreds of $\mathrm{mM}$ of drug to study their channel blocking action, when the therapeutic range lies more than 4-5 orders of magnitude lower ${ }^{452}$. We therefore attempted to use clinically valid concentrations, to obtain a more realistic correlation with what could possibly be clinically observed.

\subsection{1. $A P D_{x x}$ Prolongation and Increased $A P D_{x x}$ Dispersion $\left(\triangle A P D_{x x}\right)$ with Flecainide in the Murine Heart}

Flecainide has been reported to cause $\mathrm{APD}_{90}$ prolongation in mammalian ventricular myocytes 479 , 635 , as well as human, guinea pig, and canine atria636, 637. However it was also shown to cause APD 90 shortening in canine Purkinje fibers ${ }^{635}$. In our measurements, APD prolongation was observed at all

Ixxix Manual of Laboratory \& Diagnostic Tests. $7^{\text {th }}$ edition. Lippincott Williams \& Wilkins. 
repolarization levels $\left(\mathrm{APD}_{25}, \mathrm{APD}_{50}\right.$ and $\left.A P D_{75}\right)$ with Flecainide (Figure 30, Figure 32, Figure 42), and most prominently in $\mathrm{APD}_{25}$ measurements (Figure 34, Figure 43) in all substrates (WT, $m d x$, and $\triangle \mathrm{KPQ}$ ). This suggests that with an increasingly shallower upstroke, the amplitudes and kinetics of sequential ionic currents could be modified, causing shifts in the phases of the epicardial AP with respect to voltage and time. In reality, with the strong use-dependence of Flecainide, APD prolongation was not expected. Since our experiments were conducted using only one frequency of stimulation, carried at steady state, we cannot comment on how the rate of APD prolongation would change, if different frequencies were involved, neither can we speculate on the restitution behavior of the APD in the presence of Flecainide. Although we have observed an APD prolongation in all sinus measurements (both WT and $m d x$ ) with Flecainide compared to the starting control conditions, this finding is not reliable because the measured APDs are at different locations on the restitution curve (due to the varying diastolic interval between initial control conditions ( $\mathrm{t} 0$ ) and after Flecainide was introduced into the system). At control conditions, the sinus rate was no less than $8-8.3 \mathrm{~Hz}$ (i.e. $480-500 \mathrm{bpm}^{\mathrm{lxxx}}$ ). After ten minutes of Flecainide exposure, the sinus rate dropped to a value between $4.5-5.8 \mathrm{~Hz}(270-350 \mathrm{bpm})$ in almost all preparations. Hence, we cannot confirm whether the observed APD prolongation is secondary to pure Flecainide effects or due to the final lower heart rates.

The observation that Flecainide causes an actual prolongation of the APD doesn't correlate well with the fact that this drug has a strong use-dependence block ${ }^{479}$. Indeed, Flecainide is unique among AADs, where use-dependence block of $\mathrm{Na}_{v} 1.5$ causes a prolongation of the APD and not retraction ${ }^{499,638}$. This might suggest that Flecainide blocks other channels than just Nav1.5. Follmer et al. showed that Class Ic agents have a markedly different ionic profile among $\mathrm{Na}_{\mathrm{v}} 1.5$ blockers: Flecainide has the potential to block the rapid component of the delayed K-rectifier current $\left(\mathrm{I}_{\mathrm{Kr}}\right)$ using concentrations as low as $2.1 \mu \mathrm{M}$ in cat ventricular myocytes 639 . Although the concentration used in the latter experiments is at least twice as large as the one used with our murine hearts, we cannot eliminate the possibility that blocking $\mathrm{K}^{+}$channels could be involved in the observed APD prolongation with Flecainide.

In a previous study done by Starmer et al., they showed that prolonging refractoriness by blocking $\mathrm{Na}_{v} 1.5$ alone increases the likelihood of unidirectional block in the presence of premature excitations in an otherwise normal tissue ${ }^{492}$. Follmer et al. showed that the interaction of Flecainide with $\mathrm{K}^{+}$channels closely correlated with channel activation, suggesting an open-state blocking effect ${ }^{639}$. As the result of the two previous findings, Follmer et al. argue that the antiarrhythmic efficacy of Flecainide resides in its ability to act on both $\mathrm{Na}_{\mathrm{v}} 1.5$ and $\mathrm{K}^{+}$channels, by prolonging refractoriness in the setting of decreased excitability, without the need to solely reduce $\mathrm{I}_{\mathrm{Na}, \mathrm{f}}$ in order to achieve the same degree of refractoriness 639 .

APD prolongation is of different magnitude for different levels of repolarization, with Flecainide. For higher repolarization levels for which the APDs were originally defined (i.e. the closer temporally is the repolarization level to the upstroke), the more prominent is the percentage increase in prolongation observed between $t_{0}$ and $t_{10}$, in either WT or $m d x$ (Figure 34 ). Both APD $_{50}$ and $\mathrm{APD}_{75}$ showed no directional bias, in such a way that the percentage increase didn't vary whether the APs were picked up along the longitudinal or transversal directions. To illustrate, in the WT APD 50,Long prolonged by $\sim 65 \%$ and $\mathrm{APD}_{50 \text {,Trans }}$ by $\sim 60 \%$. Although the percentage increases in the $m d x$ were slightly higher (but not statistically significant) compared to the WT, no directional preferences were detected; $\mathrm{APD}_{50}$, Long and $\mathrm{APD}_{50 \text {,Trans }}$ prolonged by $\sim 70 \%$ and $\sim 69 \%$ respectively. $\mathrm{APD} \mathrm{D}_{75}$ and $\mathrm{APD}_{90}$ in both WT and $m d x$ prolonged overall by $\sim 40 \%$ and $\sim 30 \%$ respectively in both directions.

Ixxx In cardiology, the heart rate is measured in bpm, i.e. beats per minute. 
The dynamics of $\mathrm{APD}_{25}$ were dramatically different than other APDs. This parameter appeared to be the most susceptible to Flecainide, acquiring both direction- and substrate-dependences (Figure 34) as the treatment progressed. In the WT, the prolongation of $\mathrm{APD}_{25}$ peaked along the longitudinal direction with an increase exceeding $\sim 190 \%$, vs. $\sim 170 \%$ in the transversal direction. The $m d x$ heart, with lower available $\mathrm{Na}_{v} 1.5$ channels, showed significantly lower percentages of $\mathrm{APD}_{25}$ increase between $t_{0}$ and $t_{10}$ upon exposure to Flecainide, with $\sim 155 \%$ and $\sim 110 \%$ in the longitudinal and transversal directions, respectively. These data emphasize three important findings:

- As $\mathrm{APD}_{25}$ encloses the time interval from AP takeoff till the end of phase 1 , it represents the interval during which the dynamical processes the most affected by functional $\mathrm{Na}_{1} 1.5$ take place. In other words, this correlates well with the fact that under control conditions, only $\mathrm{APD}_{25}$ showed a significant difference between WT and $m d x$, whereas other APDs were not statistically different (Figure 30, Figure 32). In our simplistic assumptions, the major difference in the plasma membrane ionic composition between WT and $m d x$ is the absence of Nav1.5 on the LM in the mutated heart. Hence, if subsequent ionic currents were perturbed in the $m d x$ compared to the WT in the earliest phases of repolarization (in the absence of Flecainide), they should stem from the differences in Nav1.5 availability between the two substrates (Figure 19).

- Flecainide enhanced the appearance of directional bias only in $\mathrm{APD}_{25}$, whether in the WT alone (by comparing longitudinal to transversal directions), in the $m d x$ alone or when comparing the two substrates. The lower percentage increases in $\mathrm{APD}_{25, m d x}$ (compared to $\mathrm{APD}_{25, \mathrm{WT}}$ ) further underline that the lack of $\mathrm{Na}_{\mathrm{V}} 1.5$ on the LM means lower available channels to modulate. Henceforth, the total change induced by Flecainide in either direction is expected to be reduced in the $m d x$ (as it was indeed observed in the statistics in Figure 34).

- The increased susceptibility of $\mathrm{APD}_{25}$ to Flecainide (compared to $\mathrm{APD}_{50}, \mathrm{APD}_{75}$ or $\mathrm{APD}_{90}$ ) highlights that in our experiments, whether $[1 \mu \mathrm{M}]$ Flecainide also blocks $\mathrm{K}^{+}$channels, the main functional effect of the drug is localized to high enough phases of the AP, where repolarizing $\mathrm{K}^{+}$channels play practically no role.

Because of the fact that $\mathrm{APD}_{25}$ incorporates other AP processes beyond the upstroke phase, its sensitivity to directional influences is less pronounced than the one observed for the maximum upstroke velocity $(\mathrm{dF} / \mathrm{dt})_{\max }$ (Figure 25), where the decay in $(\mathrm{dF} / \mathrm{dt})_{\max }$ was similar between WT and $m d x$ along the longitudinal direction, but more pronounced in the WT along the transversal direction (compatible with the differences in Nav1.5 cellular distribution between the two substrates). Hypothetically speaking, if $\mathrm{APD}_{25}$ were to represent the phase of the AP where Nav1.5 is the only functional channel, then the behavior of $\mathrm{APD}_{25}$ and $(\mathrm{dF} / \mathrm{dt})_{\max }$ with Flecainide at any time point of the treatment with Flecainide should exactly match. Since the latter statement is precisely not true, as Flecainide exposure proceeded, the coupling between $\mathrm{ADP}_{25}$ and $(\mathrm{dF} / \mathrm{dt})_{\max }$ became increasingly ambiguous, indicating a decoupling between the two parameters (Figure 35). This could suggest that as the upstroke velocity becomes progressively weaker, under the influence of the drug, the ability to Nav1.5 to orchestrate the sequential activation of the consecutive channels becomes increasingly more limited (Figure $35 \mathrm{~b}$ ). At $\mathrm{t}_{10}$, further deterioration of the upstroke would hinder the capacity of Nav1.5 to even coordinate the earliest phases of the AP (particularly the ones involving $\mathrm{I}_{\mathrm{to}, 1}$ and $\mathrm{I}_{\mathrm{Ca}, \mathrm{L}}$ ) after the upstroke; a mechanism that could promote electrical instability in the tissue.

The spatial distribution of the above macroscopic processes couldn't have been elucidated using traditional microelectrodes positioned at few sites of the epicardium. The strength of the observed findings relies in the methodology used to dissect both spatially and temporally the effects related to the propagating electrical activity across the entire epicardium. Furthermore, without optical 
mapping, the increase in spatial dispersion of repolarization (SDR) couldn't have been described with the precision adopted in this work. Flecainide not only induced a differential prolongation in the APD values, but also in the spatial organization of these APDs across the epicardium, where WT and $m d x$ hearts both showed a pronounced increase in their SDR at different AP repolarization (Figure 33). Under control conditions (i.e. when no Flecainide was introduced), $\mathrm{APD}_{25}$ was not only significantly prolonged in the $m d x$, but also the dispersion of the $\mathrm{APD}_{25}$ (i.e. $\Delta \mathrm{APD}_{25}$ ) was significantly widened in the $m d x\left(\Delta \mathrm{APD}_{25, \mathrm{WT}}=8.4 \pm 1.7 \mathrm{~ms}, \Delta \mathrm{APD}_{25, m d x}=13.1 \pm 4.0 \mathrm{~ms}, p\right.$-value $\left.<0.01\right)$. Although $\triangle \mathrm{APD}_{25, m d x}$ was higher on average $(29.3 \pm 7.1 \mathrm{~ms})$ than $\triangle \mathrm{APD}_{25, \mathrm{WT}}(25.6 \pm 6.6 \mathrm{~ms})$, bistability erupted in at least $70 \%$ of the WT hearts $(\mathrm{n}=10)$ at $\mathrm{t}_{10}$ and in $0 \%$ of $m d x$ heart $(\mathrm{n}=7)$. The emergence of bistability in the $\mathrm{APD}_{50}$ profile in the WT heart at $\mathrm{t}_{10}$ led to the significance increase in dispersion (Figure 33). Hence, we believe that the $\triangle \mathrm{APD}_{50, \mathrm{wT}}$ and $\triangle \mathrm{APD}_{75, \mathrm{wT}}$ widening at $\mathrm{t}_{10}$ is a consequence of bistability, rather the causative agent behind it.

To further illustrate why this point could be crucial in our understanding of symmetry breaking in APD, the same analysis of APD prolongation and dispersion was carried out in the $\triangle \mathrm{KPQ}$ model treated with Flecainide $[1 \mu \mathrm{M}]$ and their WT control, over a protocol that lasted for $5 \mathrm{~min}$ (due to the premature loss of steady state conditions with the $\triangle \mathrm{KPQ}$ model beyond $5 \mathrm{~min}$ of treatment). Although the spatial organization of APDs in the $\triangle \mathrm{KPQ}$ heart doesn't show the same sharp transition observed previouslylxxi, the heterogeneous distribution of $\mathrm{APD}_{25}$ and $\mathrm{APD}_{50}$ at $\mathrm{t}_{5}$ indicates the presence of steep gradients between the distinct zones observed on the epicardial surface (Figure $39 \mathrm{a}$ and b). Although $\triangle \mathrm{APD}_{50, \mathrm{WT}}$ was significantly higher in the WT at $\mathrm{t}_{5}$ compared to $\triangle \mathrm{APD}_{50, \Delta \mathrm{KPQ}}$, symmetry breaking emerged in the $\triangle \mathrm{KPQ}$ and not the WT (the difference in $\mathrm{APD}_{50}$ values is not significantly different between the two groups, Figure 42). We believe that since we are principally targeting $\mathrm{Na}_{v} 1.5$ with Flecainide, the mechanism by which modulation of Nav1.5 occurs is capable of influencing the subsequent events leading to symmetry breaking. We hypothesize that in the presence of Flecainide, neither $\mathrm{APD}_{25}$ prolongation nor widening of $\triangle \mathrm{APD}_{25}$ alone may evoke symmetry breaking in a well-coupled tissue. However, cumulatively, whenever the global change in $\triangle \mathrm{APD}_{25}$ with Flecainide exceeds the overall prolongation in $\mathrm{APD}_{25}$, the substrate becomes more susceptible to symmetry breaking (indicated by the statistics in Figure 43). The latter statement is by far just a hypothesis based on an observation from our experiments, further investigations using computer models of the mouse heart will be required to find out, whether symmetry breaking with Flecainide could occur under the pre-requisites set above.

The concept of heterogeneous repolarization, as a facilitator of arrhythmogenic activity, has been recognized for more than four decades $407-409$. When transmembrane repolarization at some sites of the cardiac tissue outlasts repolarization at an adjacent site, local current will be expected to flow in proportion to the voltage gradient between the two sites ${ }^{411}$. If the current is of sufficient magnitude, it can reexcite the earlier repolarizing site by bringing the cells' potential to threshold triggering reentry 264. The prominent increase in SDR observed in our murine hearts is likely to be arrhythmogenic because the dispersion in repolarization occurs over very short distances 264 (approximately, the apico-basal length of the murine heart is $\sim 6 \mathrm{~mm}$ and the width of the LV free wall in our field of view $\sim 3 \mathrm{~mm}$ ), creating a very steep repolarization gradient (in the example in Figure 28a, the gradient can exceed $20 \mathrm{~ms}^{-\mathrm{mm}^{-1}}$ across the borderline from the zone with the prolonged APDs to the zone of the abbreviated ones). It's currently believed that the steepness of the repolarization gradient, rather than the total magnitude of the dispersion in a cardiac tissue, determines the arrhythmogenic susceptibility of the substrate involved 263,264 .

\footnotetext{
lxxxi i.e. in the WT control group of the $m d x$ mice.
} 


\subsubsection{Induction of Bistability by Flecainide in the Murine Heart}

The striking bistability emerged in the murine LV (Figure 28a, second row, right) at $\mathrm{t}_{10}$ exclusively in the WT: The epicardium appears to accommodate two juxtaposed regions with a steep repolarization gradient exceeding $40 \mathrm{~ms}$, in response to the same steady-state entraining frequency. This pattern formation is independent of anisotropy: the activation pattern of the spreading electrical wave at $t_{10}$ hasn't deviated from anisotropy (Figure 20b, left). Moreover, sinus measurements showed the same spatial configuration of the prolonged and abbreviated APDs (as in their $10 \mathrm{~Hz}$ paced counterparts) once the pattern is formed at $t_{10}$. This indicates that in our study, the bistability observed in APD behavior is a genuine characteristic of the WT substrate exposed to Flecainide, which is independent of frequency of stimulation and the nature or pattern of activation spread.

Moe and Mendez argued that dramatic differences in APD could not occur between well-coupled cells ${ }^{640}$, because electrotonic influences and wave front collision sites act to level out any intrinsic heterogeneity in AP morphology over a large spatial extent ${ }^{640,641}$. Others also suggested that abrupt transitions in APD to be possible only in poorly coupled cells ${ }^{557}$ and if electrotonic interactions were too weak to smooth out the APD, they are also likely to be as weak not to sustain a reexcitation process ${ }^{640}$. Sampson and Henriquez investigated the role of heart size, tissue properties and spatial distribution of different types of cells on functional APD dispersion ${ }^{642}$. They demonstrated in a simulation study, that electrotonic modulation of APD tends to dominate the intrinsic differences in cell properties in the small mouse heart642. They also showed that intrinsic heterogeneity in membrane properties is more prominent when the heart becomes significantly larger ${ }^{642}$. Using the Luo-Rudy mammalian model in a three dimensional model of mouse heart geometry, they showed that an intrinsic transmural variation in APD of $30 \mathrm{~ms}$ was gradually smoothed out across the ventricular wall, without giving rise to any functional heterogeneity in the medium. Their simulations were in concordance with Knollmann et al., who reported no significant differences in $\mathrm{APD}_{90}$ were observed transmurally in the mouse heart, despite prior findings that revealed significant cellular heterogeneity in isolated cells ${ }^{521}$. They concluded that functional gradients of APD in small hearts are difficult to create and that the mouse heart will not exhibit appreciable APD dispersion unless in the setting of significant uncoupling. Consequently, they concluded that the mouse heart could be a poorer model for reproducing arrhythmia mechanisms that rely on SDR ${ }^{642}$.

Using high-resolution optical mapping techniques, our experimental study demonstrates that a network of homogeneously (LV free wall only) well-coupled murine epicardial cells in intact heart preparations can simultaneously accommodate multistable excitatory states, where adjacent zones of heterogeneous repolarization properties with a gradient exceeding $40 \mathrm{~ms}$ can coexist. We showed that Flecainide, a Nav1.5 blocker agent used in clinically valid conditions, has the potential to induce multistability in a normal cardiac tissue with perfectly homogeneous electrophysiological properties at control conditions. By resolving electrical activity down to a length scale of $100 \mu \mathrm{m}$ and a frame rate of $0.5 \mathrm{~ms}$, we measured the different electrophysiological components including excitability, CV, APD and SDR, in order to characterize with significantly higher accuracy the electrical instabilities that could arise in treating a mouse heart with Flecainide. This bistability was observed exclusively in the WT mouse hearts. These results highlight the fact that the electrophysiological changes induced by Flecainide occur in a healthy murine heart and are responsible for complex pattern formation. On the other hand, the $m d x$ heart appears resistant to these instabilities at $\mathrm{t}_{10}$. We believe that the electrophysiological dynamics in the $m d x$ mouse were not favorable for symmetry breaking to occur. The role of $\mathrm{APD}_{25}$ prolongation and $\triangle \mathrm{APD}_{25}$ in predicting the occurrence of symmetry breaking in a murine cardiac tissue was described in section 
4.3.1 (p. 134). Nevertheless, using only these functional data, we cannot delineate the exact mechanism that hindered the appearance of symmetry breaking with Flecainide in the $m d x$ heart.

This symbiosis between slowed conduction and heterogeneous repolarization would be of a particular importance in pathological diseases of the heart ${ }^{483}$, where structural heterogeneity can amplify the predisposition of cardiac patients to drug-induced arrhythmias 485 , 486, in concordance with the outcomes of CAST I ${ }^{474}$. In a normal epicardium, bistability is expected to give rise to reentrant patterns in the presence of premature stimuli ${ }^{497}$. These data support the hypothesis that electrophysiological heterogeneity upon exposure to Flecainide could play a critical role in the proarrhythmic effects of the drug ${ }^{411}$. Given that arrhythmias are multicellular ${ }^{292}$, it becomes legitimate to question the efficacy and applicability of antiarrhythmic drugs as a primary mean of patients' management, when their outcomes were established based on isolated "single" cell measurements 643,644 .

\section{3•3.Hypothetical Mechanisms Behind Bistability and Arrhythmogenesis}

The current experimental outcomes with Flecainide motivated a search for a possible mechanism that could potentially account for the observed bistability in healthy tissue. Cardiac excitation involves local regenerative processes at the individual cell level and transmission of this transient dynamic process from one cell to another via intercellular connections through the flow of depolarizing charges. However, spread of excitation from one point to another in the cardiac tissue occurs exclusively when a critical "amount" of cells (source) is simultaneously excited, as to generate a depolarizing current sufficient to overcome the sink provided by the quiescent tissue in order to bring downstream cells to threshold645. Therefore, several cells below this critical mass cannot entrain its neighbors to acquire the same characteristics. Given that myocytes in an intact tissue are well coupled to their neighbors, how do these cells synchronously develop two almost opposing dynamics in such a small sized heart?

We hypothesize about the generic mechanism that could have lead to the bistability observed in our experiments. We start by considering two observed properties of bistability: First, bistability is not dependent on anatomy or structure and its spatial configuration, whenever it appears, was not conserved across preparations (i.e. among the WT preps). Second, WT hearts that eventually showed a steep repolarization gradient with Flecainide and the $m d x$ hearts that failed to do so, shared similar APD distributions at initial conditions (i.e. in the absence of the drug). Henceforth, we are reluctant to associate bistability to differences in the ionic machinery of individual cells, which was shown by Szentadrassy et al. to account for apico-basal differences in APD in the canine ventricle ${ }^{646}$. We, therefore, make the assumption that all cells of the $\mathrm{LV}$ free wall epicardium are homogeneously equipped with the same ionic channels. The first possibility assumes that each and every individual cell has the capacity to either acquire a long or a short APD. The presence of two adjacent zones with a dominating APD on each side suggests that cells, when present in a tightly coupled mesh, have the potential to help their neighboring cells deciding on which APD to display, creating finally two zones (they could be hypothetically more than just two) with each zone sharing similar electrophysiological characteristics. Another possibility assumes the presence of "leader cells" (that form a "critical mass" as explained earlier), which pick the initial conditions (i.e. a long or a short APD) and force the remaining mass of cells in the mesh to follow their lead. The emphasis in either hypothetical behavior is on these tight electrotonic interactions that overturn the cells in a multistable system to acquire one particular electrophysiology over another. Since we are not aware of the intrinsic dynamics occurring at the level of one cell, we cannot predict which possibility is more dominant. 
From an electrophysiological perspective, we hypothesize that Flecainide-induced bistability in the murine epicardium could originate from competing currents (one outward repolarizing and another inward depolarizing) acting between the end of phase 0 (i.e. the upstroke) and before the phase 2 of the AP (i.e. the plateau) gains momentum. The reason for this assumption is simple: bistability was prominent after 25\% AP repolarization, and wasn't detectable prior to this level (Figure 28, Figure $31)$. The two competing currents are $\mathrm{I}_{\mathrm{to}, 1}$ and $\mathrm{I}_{\mathrm{Ca}, \mathrm{L}}$. The idea behind the "competing currents" hypothesis is similar to the elegantly presented work by Weiss et al. in Heart Rhythm (2010) ${ }^{308}$, which provided a consistent explanation for the dynamics of afterdepolarizations at the end of the plateau phase, using a rabbit heart numerical model.

The bidirectional influence of membrane voltage and $\mathrm{Ca}^{2+}$ on each other is well documented in the literature 647,648 . In a murine or rat ventricular myocyte, $\mathrm{Ca}^{2+}$ homeostasis is strictly controlled via uptake in the sarcoplasmic reticulum or SR (more than $95 \%$ of the cytoplasmic $\mathrm{Ca}^{2+}$ available for mechanical contraction is taken up by the SR, the remaining $5 \%$ through plasma channels) ${ }^{113}$. We consider first the dynamics of $\mathrm{Ca}^{2+}$ entry into the cell and its intricate modulation by the $\mathrm{I}_{\mathrm{to}, 1}$ current. Although the $\mathrm{I}_{\mathrm{Na}, \mathrm{f}}$-dependent rapid depolarization activates $\mathrm{I}_{\mathrm{Ca}, \mathrm{L}}$, the amplitude of $\mathrm{I}_{\mathrm{Ca}, \mathrm{L}}$ doesn't reach its maximum at the peak and consequently doesn't contribute substantially to the upstroke of the AP. Bers explains that this phenomenon is partly due to the fact that the activation of $\mathrm{I}_{\mathrm{Ca}, \mathrm{L}}$ is not just voltage-dependent but also acquires some intrinsic time scale of activation, leading to a trade-off in the co-existence of relatively high channel conductance $\left(\mathrm{g}_{\mathrm{Ca}}\right)$ and low driving force $\left(\mathrm{E}_{\mathrm{mbr}}-\mathrm{E}_{\mathrm{Ca}}\right)_{\text {peak, }}$ with $\mathrm{I}_{\mathrm{Ca}, \mathrm{L}}$ being the product of these two components ${ }^{649}$. In reality, at the AP overshoot, the membrane voltage is $\sim 30-40 \mathrm{mV}$, which is relatively close to the reversal potential of $\mathrm{Ca}^{2+}\left(\mathrm{E}_{\mathrm{Rev}, \mathrm{Ca}}\right)$, which is measured under physiological conditions to be $\sim 50-60 \mathrm{mVlxxxii}^{\mathrm{l}}$, so the driving force of $\mathrm{Ca}^{2+}$ is low by the end of phase 0 . As the $\mathrm{Na}_{\mathrm{V}} 1.5$ channels inactivate and the $\mathrm{I}_{\mathrm{to}, 1}$ has already activated, phase 1 lowers the membrane potential, increasing the driving force of $\mathrm{Ca}^{2+}$ into the cell. In conclusion, $\mathrm{I}_{\mathrm{Ca}, \mathrm{L}}$ activates over two phases: A rapid increase in conductance followed by an increase in the driving force, with $\mathrm{I}_{\mathrm{to}, 1}$ directly modulating $\mathrm{Ca}^{2+}$ influx ${ }^{649}$. Inactivation of $\mathrm{I}_{\mathrm{Ca}, \mathrm{L}}$ is both voltage and $\mathrm{Ca}^{2+}$ dependentlxxxiii, with the latter being a far more predominant component of inactivation ${ }^{\mathrm{lxxxiv}}$, hence $\mathrm{I}_{\mathrm{Ca}, \mathrm{L}}$ inactivation is extremely slow ${ }^{113}$.

On the other hand, $I_{t o, 1}$ is not a voltage dependent current only, but also acquires outward rectification properties ${ }^{107}$, which means that the amplitude of this current is strongly dependent on the amplitude and velocity of the preceding upstroke, in other words on $\mathrm{I}_{\mathrm{Na} \text {,f. }}$ The sizeable amplitude of this current in rat and mouse ventricular cells abbreviates the APD and reduces the plateau phase substantially113, 650. A decrease in $\mathrm{I}_{\mathrm{Na}, \mathrm{f}}$ in ventricular cells is expected to lower the intensity of $\mathrm{I}_{\mathrm{to}, 1}$, henceforth decrease the early repolarization phase. This implies that the plateau will start at higher membrane voltages than usual, an effect which might decrease the driving force of $\mathrm{Ca}^{2+}$ into the cell, potentially altering $I_{C, L}$ peak in amplitude and time. The intricate balance in time (and within a limited range of membrane voltages) between $\mathrm{I}_{\mathrm{to}, 1}$ and $\mathrm{I}_{\mathrm{Ca}, \mathrm{L}}$ peaks has the potential to tip the intrinsic dynamics of the cells into one stable trajectory or another. In the case of the observed APD bistability in our measurements, one stable state is APD prolongation, the other APD abbreviation.

Ixxxii $\mathrm{E}_{\mathrm{Rev}, \mathrm{Ca}}$, the reversal potential of $\mathrm{Ca}^{2+}$ in physiological experiments is measured to be $\sim 50-60 \mathrm{mV}$, in contrast to its thermodynamical equilibrium potential $\left(\mathrm{E}_{\mathrm{Ca}}\right.$ ), which is $\sim 120-125 \mathrm{mV}$. Substantial amount of $\mathrm{Ca}^{2+}$ flows across the $\mathrm{L}-\mathrm{typ}$ e channels between ERev,Ca and ECa. But in a real cell at ERev,Ca the inward ICa,L is counterbalanced by the outward K-current

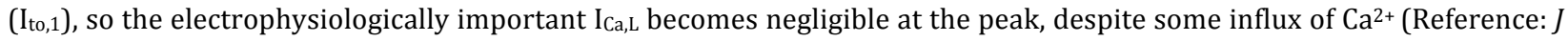
Physiol (2000) 523:57-66).

lxxxiii Refer to Introduction section 1.1.4 on $\mathrm{Ca}^{2+}$ inactivation.

lxxxiv Since the predominant factor in $\mathrm{Ca}^{2+}$ inactivation is metabolic, this process is considered relatively slow compared to inactivation of other currents which are completely voltage or time dependent (like $\mathrm{I}_{\mathrm{Na}}$ and $\mathrm{I}_{\mathrm{to}, 1}$ ). 
Henceforth we hypothesize that by lowering $\mathrm{I}_{\mathrm{Na}, \mathrm{f}}$ with Flecainide and continuously reducing its rate of increase as the treatment progresses, $\mathrm{I}_{\mathrm{to}, 1}$ activation and inactivation processes are also altered. A plateau phase is sustainable whenever repolarizing currents ( $\mathrm{K}^{+}$currents) are not fully activated and depolarizing currents still didn't inactivate $\left(\mathrm{I}_{\mathrm{Ca}, \mathrm{L}}\right)$. Following the upstroke phase, if $\mathrm{I}_{\mathrm{to}, 1}$ peaks early enough, it will lower the membrane voltage and allow for the increase in $\mathrm{Ca}^{2+}$ driving force. Ito,1 inactivates very quickly, allowing $\mathrm{I}_{\mathrm{Ca}, \mathrm{L}}$ to peak at a later stage as $\mathrm{Ca}^{2+}$ rush into the cell, which would ultimately keep the plateau phase at higher voltages. As Flecainide also affects $\mathrm{I}_{\mathrm{Kr}}$, the current would be further weakened to counteract $\mathrm{Ca}^{2+}$ influx and the plateau is substantially increased. If on the other hand, $\mathrm{I}_{\mathrm{to}, 1}$ activation is delayed enough to peak at a later stage, phase 1 is prolonged and $\mathrm{Ca}^{2+}$ influx could face a fierce competing opposing current as it's trying to reach its maximum. This would obliterate the plateau and bring the cell into resting potential, abbreviating the APD.

This hypothesis on competing currents following the upstroke has to await further experiments and numerical simulations to be approved as valid or disapproved. One way to elucidate the role of $\mathrm{I}_{\mathrm{to}, 1}$ would be to disable it by introducing 4-aminopyridine (4-Ap) into the protocol, which could provide important and specific insights on the role played by $\mathrm{I}_{\mathrm{to}, 1}$ in creating this bistability in the epicardium. We expect that the presence of this antagonist molecule may potentially alter the responsiveness of the epicardium to Flecainidel ${ }^{\mathrm{xxxv}}$ or even prevent bistability from occurring altogether. Alternatively, hypercalcemia may favor one stable state over another, however it can alter other $\mathrm{Ca}^{2+}$ dependent mechanisms in the cell, which could render the final results harder to interpret. Therefore, the detailed explanations given in this section should only be regarded as one potential mechanism explaining the emergence of bistability in the murine heart.

Although our study was limited to steady state pacing at a fixed BCL (100ms), we hypothesize that a premature excitation occurring in the epicardium would give rise to drug-induced arrhythmias that are characterized by a reentrant mechanism. The mechanism by which reentry is initiated would depend on the specific location of the ectopic beat with respect to the different zone of AP morphology. APD prolongation goes hand in hand with repolarization abnormalities, hence is capable of influencing conduction of the propagating impulse. Fibers with prolonged APD will elicit a slowing of conduction with increased entrainment rate, so that each consecutive AP arises prior to the completion of repolarization of the preceding beat. The frequency at which conduction block might ensue will be relatively lower in the case of the prolonged APD, than the one associated with a normal APD ${ }^{318}$. If the ectopic beat arises in the area governed by shorter APDs (APD ${ }_{\text {Short}}$ ), the electrical wave would spread across the tissue, until it hits the borders separating the two adjacent zones. Crossing to the prolonged APD zone (APD Prolong) will depend on the excitatory state of the cells in that region: if theses cells had sufficient time to recover from the previous excitation, the wave will propagate. Otherwise, conduction block will occur. In this setting, either the electrical wave dies out or persists somewhere else until the cells in the APDProlong zone regain excitability. In this case, circus movements are highly probable and reentry occurs. If however the ectopic beat

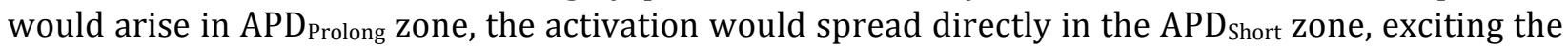
cells in that region. Interestingly, for reexcitation to occur, sustenance of propagation would then depend on the electrotonic interactions between the cells on either side of the borders. It has been suggested that in tissues where depolarization is normally Na-mediated, the contribution of $\mathrm{Ca}^{2+}$ in maintaining AP propagation increases significantly in the context of severely depressed membrane excitability (i.e. $<20 \% \mathrm{I}_{\mathrm{Na}}$ availability) ${ }^{585}$. In the case of Flecainide induced heterogeneity, it is the prominent plateau at some sites ( $A$ PD $\left.D_{\text {Prolong }}\right)$ but not others ( $A P D_{\text {Short }}$ ) that could generate local

lxxxv The effects due to antagonizing Ito,1 using 4-AP were described by Krishnan and Antzelevitch in epicardial layers in the presence of Flecainide. Ito,1 blocking was reported to prevent AP abbreviation in epicardial cells (Reference: Circulation (1993) 87:562-72). 
circuit currents ${ }^{411}$, by acting as a reservoir of depolarizing charges $\left(\mathrm{Ca}^{2+}\right)$, which would increase the electrotonic driving force in the abbreviated AP cells, consequently, the depolarizing current to the downstream tissue ${ }^{585}$, bringing membrane voltage to threshold and initiating depolarization. Kléber and Rudy described this membrane-switch from $\mathrm{Na}^{+}$to $\mathrm{Ca}^{2+}$-supported propagation as an excellent example of the "intimate interaction" between passive network properties and the excitatory membrane currents during conduction in the cardiac tissue ${ }^{254}$. Whether this switch in membrane conduction is sufficiently strong to maintain a self-perpetuating reentrant mechanism in the heart in the context of bistability is yet to be investigated.

\subsubsection{Inflation of Intrinsic Heterogeneity in LQTS3 with Flecainide}

As $\mathrm{Na}_{v} 1.5$ kinetics are altered in the context of the $\triangle \mathrm{KPQ}$ mutation, exposing a cardiac tissue harboring this mutation to Flecainide had two primordial aims: First, studying the effect of the drug on intrinsic spatial heterogeneity in the context of a well-established arrhythmogenic substrate. Second, characterizing whether the symmetry breaking is reproducible in a substrate with a gain of function-channel. Our data clearly demonstrate that a typically anisotropic propagation in the tissue (Figure $36 \mathrm{~b}$ ) at control conditions ( $\mathrm{t}_{0}$ ) deteriorated in $5 \mathrm{~min}$ (half the protocol time used for the $m d x$ and its WT counterpart in section 4.3.1) into an atypical propagating pattern that deviates from anisotropy, associated with areas of fast and slow conductions (Figure 36c). Despite a severe CV decrease $\left(>50 \%\right.$ along $\left.V_{\min , \Delta K P Q}\right)$ on the apico-basal axis, conduction block wasn't observed in the $\triangle \mathrm{KPQ}$ hearts under steady state pacing entrainment conditions in any direction.

From the initial comparison of AP morphologies (in the absence of Flecainide), where $\mathrm{AP}_{\triangle \mathrm{KPQ}}$ is comprehensively prolonged compared to $\mathrm{AP}_{\mathrm{WT}}$ at all stages of repolarization (Figure 36a), it became evidently expected that all APDs $\left(A P D_{25}, A P D_{50}\right.$ and $\left.A P D_{75}\right)$ show a significant prolongation in the $\triangle \mathrm{KPQ}$ tissue compared to its WT counterpart (Figure 42). Unlike the global increase in APD in the $\triangle \mathrm{KPQ}$ heart, dispersion of repolarization is particularly significant for higher repolarization levels compared to the WT (i.e. only for $\triangle \mathrm{APD}_{25}$, Figure 41). In the presence of Flecainide, APD values increased in both WT and $\triangle \mathrm{KPQ}$ degrading the statistical significance of the control findings (Figure 42). Furthermore, both WT and $\triangle \mathrm{KPQ}$ responded to Flecainide by an increase in SDR within the ventricular epicardium for both $\mathrm{APD}_{25}$ and $A P D_{50}$ (Figure 41). The particular accentuation of $\triangle \mathrm{APD}_{25}$ with Flecainide in the $\triangle \mathrm{KPQ}$ heart provides solid evidence that the abnormal increase in SDR, due to the mutation, is not corrected by Flecainide. In reality, our data show that in addition to the atypical complex spatial-temporal patterns of activation in the $\triangle \mathrm{KPQ}$ substrate (Figure 36), repolarization is exceedingly destabilized in the presence of Flecainide. Consistent with our previous argument (section 4.3.2), the cumulative effect of the increases in $A P D_{25}$ and $\triangle A P D_{25}$ from their initial control values favored symmetry breaking in the $\triangle \mathrm{KPQ}$ heart and not the WT, even though the increase in $\triangle \mathrm{APD}_{25}$ was significantly more considerable in the WT, compared to the one in $\triangle \mathrm{KPQ}$ (Figure 41 ).

These data show that Flecainide could be dangerous in LQTS3 patients, particularly the one harboring the $\triangle \mathrm{KPQ}$ mutation. The ever-increasing complexity of the interactions between mutated channels and non-ideal antiarrhythmic drugs muddles the ability to predict the benefits, if any, of managing LQTS3 patients with Flecainide. Clinical reports on this level remain conflicting. Some studies have shown that Flecainide has potentially therapeutic effects, since it was associated with the normalization of the $\mathrm{QT}_{\mathrm{c}}$-interval on ECG567, 569. Although the pathognomonic sign of LQTS3 is having a prolonged $\mathrm{QT}_{\mathrm{c}}$ interval, some studies revealed that the transmural dispersion of repolarization is more indicative of the proarrhythmic propensity for developing of lethal ventricular arrhythmias, which is better represented by $\mathrm{T}_{\text {peak }}-\mathrm{T}_{\text {end }}$ (time interval between the peak of the $\mathrm{T}$ wave and its end on ECG) than a prolonged $\mathrm{QT}_{\mathrm{c}}{ }^{651}$. As a consequence, $\mathrm{T}_{\text {peak }}-\mathrm{T}_{\text {end }}$ (time interval between the peak of the $T$ wave and its end on ECG) was suggested to be a more sensitive 
biomarker than the $\mathrm{QT}_{\mathrm{c}}$-interval in assessing arrhythmia risk in a LQTS patient ${ }^{651}$, although a direct validation of $T_{\text {peak }}-T_{\text {end }}$ interval measured on body surface ECG is still lacking 264 . The inability to extrapolate experimental findings to clinical settings renders the interpretation of the experimental data even more complicated.

\subsubsection{Concluding Remarks}

The work presented in this thesis has provided an interesting continuum for exploring the scaling of molecular perturbations to macroscopic behaviors, linking therefore molecular events that share one common denominator the cardiac Na-channel, or Nav1.5, to patterns of activation conduction and dispersion of repolarization, in a mesh of well-coupled tissues. By characterizing a set of biophysical parameters and elucidating fundamental connections between them, we provided a generic approach to understand modulations of $\mathrm{Nav}_{v} 1.5$ by studying their macroscopic consequences; therefore integrating our knowledge of the system and the data acquired at the level of the whole organ in the comprehension of the channel's behavior in both pathological and pharmacological conditions.

This multidisciplinary approach in integrative cardiology allows us to study how genetic mutations and pharmacological interventions can possibly lead to destabilizations of the normal electrical propagation in the cardiac tissue. For this purpose, we used two mouse models harboring Nav1.5 abnormalities and we further modulated the function of this channel by means of Flecainide, a currently in-use AAD. We relied in our methodology on a high spatial-temporal tool that offers the luxury and precision of studying complex extended spatial-temporal dynamics occurring in a healthy, as well as diseased, heart. We implemented and validated different analytical strategies (some of them already published and used in optical mapping of murine hearts) to evaluate CV in the excitable tissue and to further characterize instabilities in conduction in several models where $\mathrm{Na}_{v} 1.5$ function is perturbed.

We consistently observed bistability and symmetry breaking effects in models with normal and increased channel availability. These manifestations were completely absent in the model where the few Nav1.5 channels were missing from the LM. Such a symmetry breaking effect is likely to be arrhythmogenic, especially in the presence of steep repolarization gradients. We attributed these pattern formations with Flecainide as proarrhythmic side effects, with potentially strong clinical implications: clinically valid concentrations of the drug were sufficient to induce steep repolarization gradients in the epicardium of small hearts. Henceforth, the same drug could be expected to facilitate larger spatial-temporal dispersions in larger hearts, including human hearts, where more structural heterogeneity prevails over relatively wider extents. These functional heterogeneities could be several orders of magnitude larger, therefore more dangerous and lethal. The outcomes of CAST I were only the tip of the iceberg and the deeper scientists dive to uncover the reasons why Flecainide increased mortality in the treated group, the more complex and intricate the journey becomes to understand how the heart actually works.

In this context, the battle towards safe and effective AADs continues... 
(This page is intentionally left blank) 



\section{Chapter 5}

\section{Conclusion}

Over the last century, our understanding of how the heart functions has made tremendous leaps forward, with a pivotal aim of translating this information into improved therapies. Drugs have been developed and tested rigorously and redundantly in isolated cardiac cells and showed to exhibit strong antiarrhythmic effects. Scientists thought they had these molecules figured out, until CAST and other large-scale clinical trials demonstrated that the knowledge researchers have accumulated over the last two decades before CAST has simply failed to counteract death in patients with myocardial infarction.

The reality of how far scientists were far off at the time from preventing sudden death was outrageous and indisputable. These promising molecules were not only inferior to placebo but also worse; they actually increased mortality in the treated group. Since CAST, basic medical investigators and clinicians started asking a new question: "Where did we go wrong?" It took scientists a while to realize that studying the basic functional unit was insufficient to fully characterize the effects observed in the entire cardiac tissue. Single channel events in a cell simply provide an incomplete picture of the events that could take place at the level of the whole heart. Scientists had had missed the fact that the relationship between cellular excitability and multicellular conduction is inherently non-linear, making electrophysiological cardiac events much harder to predict. We have learned in the past decades that the heart is intrinsically heterogeneous and complex in its design, mechanics and electrical properties and we have discovered that this intriguing complexity has become by itself a sufficient drive for continuous research in cardiac electrophysiology. We have gone a long way since CAST, but we are still very far from the finish line. We have always known that the human life is just too precious to be jeopardized in one single erratic beat.

In 1628, Sir William Harvey was tempted to think that only God knows how the heart truly works. I, in 2014, am tempted to believe that God never intended to keep this knowledge to Himself, that's why we have gone so far and are still heading forward. 
(This page is intentionally left blank) 


\section{Bibliography}

\section{References}

1. Sherwood L. Human physiology: From cells to systems. Brooks/Cole, Cengage Learning; 2010.

2. Craig RW, Padron R. Molecular structure of the sarcomere. In: Engel AC, Franzini-Armstrong C, eds. Myology. McGraw-Hill; 2004:130.

3. Abriel H. Roles and regulation of the cardiac sodium channel na $v$ 1.5: Recent insights from experimental studies. Cardiovasc Res. 2007;76:381-389. Epub 2007 Aug 2008.

4. $\quad$ CardioConsult. Heart anatomy. 2011

5. $\quad$ Science.com D. 3d heart conduction system. 2011

6. Schaub MC, Hefti MA, Zuellig RA, Morano I. Modulation of contractility in human cardiac hypertrophy by myosin essential light chain isoforms. Cardiovasc Res. 1998;37:381-404.

7. Grant AO. Cardiac ion channels. Circ Arrhythm Electrophysiol. 2009;2:185-194. doi: 110.1161/CIRCEP.1108.789081.

8. Skwarek M, Grzybiak M, Kosinski A, Hreczecha J. Basic axes of human heart in correlation with heart mass and right ventricular wall thickness. Folia Morphol. 2006;64:385-389

9. Carl SL, Felix K, Caswell AH, Brandt NR, Ball WJ, Vaghy PL, Meissner G, Ferguson DG. Immunolocalization of sarcolemmal dihydropyridine receptor and sarcoplasmic reticular triadin and ryanodine receptor in rabbit ventricle and atrium. J Cell Biol. 1995;129:672-682

10. Schiaffino S, Reggiani C. Molecular diversity of myofibrillar proteins: Gene regulation and functional significance. Physiol Rev. 1996;76:371-423.

11. Ulbricht W. Sodium channel inactivation: Molecular determinants and modulation. Physiol Rev. 2005;85:1271-1301.

12. Schaub MC, Hefti MA, Harder BA, Eppenberger HM. Various hypertrophic stimuli induce distinct phenotypes in cardiomyocytes. J Mol Med (Berl). 1997;75:901-920.

13. Goetz C. Jean-martin charcot (1825-1893). Journal of Neurology. 2005;252:374-375

14. Balse E, Steele DF, Abriel H, Coulombe A, Fedida D, Hatem SN. Dynamic of ion channel expression at the plasma membrane of cardiomyocytes. Physiol Rev. 2012;92:1317-1358

15. Goetz CG. Charcot, the clinician: The tuesday lessons. New York: Raven Press; 1987.

16. Crossman D, Pawley JB. Confocal micrograh of heatly cardiac tissue stained with f-actin. Handbook of biological confocal microscopy, 3rd Ed. 2006

17. Birn AE. Small(pox) success? Cien. 2011;16:591-597.

18. Mitka M. Happy 50th birthday, salk vaccine. Jama. 2005;293:1581-1583.

19. McKusick VA. A 60-year tale of spots, maps, and genes. Annu Rev Genomics Hum Genet. 2006;7:1-27.

20. Coller BS. The physician-scientist, the state, and the oath: Thoughts for our times. J Clin Invest. 2006;116:2567-2570

21. Levy MN, Pappano AJ. Cardiovascular physiology. Mosby Elsevier; 2007. 
22. de Micheli-Serra A, Iturralde-Torres P, Izaguirre-Avila R. How electricity was discovered and how it is related to cardiology. Arch Cardiol Mex. 2012;82:252-259

23. Mackenzie J. Diseases of the heart. London: Henry Frowde and Hodder \& Stockton; 1913.

24. McCormick JS. James mackenzie and coronary heart disease. J R Coll Gen Pract. 1981;31:2630.

25. Einthoven W. Ueber die form des menschlichen elektrocardiogramms. Pfluegers Arch. . $1895 ; 60$

26. Jalife J, Delmar M, Anumonwo J, Berenfeld O, Kalifa J. Basic mechanisms of cardiac arrhythmias. Basic cardiac electrophysiology for the clinician. Wiley-Blackwell; 2009:152190.

27. Mines GR. On dynamic equilibrium in the heart. J Physiol. 1913;46:349-383.

28. Wit AL, Cranefield PF. Reentrant excitation as a cause of cardiac arrhythmias. Am J Physiol. 1978;235:H1-17.

29. Mayor AG. Rhythmical pulsation in scyphomedusae. Carnegie Institution of Washington; 1906.

30. Janse MJ, van Capelle FJ, Morsink H, Kleber AG, Wilms-Schopman F, Cardinal R, d'Alnoncourt CN, Durrer D. Flow of "injury" current and patterns of excitation during early ventricular arrhythmias in acute regional myocardial ischemia in isolated porcine and canine hearts. Evidence for two different arrhythmogenic mechanisms. Circ Res. 1980;47:151-165.

31. Dhamoon AS, Jalife J. The inward rectifier current (ik1) controls cardiac excitability and is involved in arrhythmogenesis. Heart Rhythm. 2005;2:316-324.

32. Zipes DP, Wellens HJ. Sudden cardiac death. Circulation. 1998;98:2334-2351.

33. Alexander S, Kleiger R, Lown B. Use of external electric countershock in the treatment of ventricular tachycardia. Jama. 1961;177:916-918.

34. Mirowski M, Reid PR, Mower MM, Watkins L, Gott VL, Schauble JF, Langer A, Heilman MS, Kolenik SA, Fischell RE, Weisfeldt ML. Termination of malignant ventricular arrhythmias with an implanted automatic defibrillator in human beings. $N$ Engl J Med. 1980;303:322-324.

35. Myerburg RJ, Spooner PM. Opportunities for sudden death prevention: Directions for new clinical and basic research. Cardiovasc Res. 2001;50:177-185.

36. Chaudhry GM, Haffajee CI. Antiarrhythmic agents and proarrhythmia. Crit Care Med. 2000;28:N158-164.

37. Rae AP, Kay HR, Horowitz LN, Spielman SR, Greenspan AM. Proarrhythmic effects of antiarrhythmic drugs in patients with malignant ventricular arrhythmias evaluated by electrophysiologic testing. J Am Coll Cardiol. 1988;12:131-139.

38. Coronel R. Challenging cardiac electrophysiology. Frontiers in Physiology. 2010;1

39. McDonagh GK. Heart failure epidemiology: European perspective. Curr Cardiol Rev. 2013;2013:15

40. Kjekshus J. Arrhythmias and mortality in congestive heart failure. Am J Cardiol. 1990;65:42I48I.

41. Lombardi R. Genetics and sudden death. Curr Opin Cardiol. 2013;28:272-281

42. Campuzano O, Beltran-Alvarez P, Iglesias A, Scornik F, Perez G, Brugada R. Genetics and cardiac channelopathies. Genet Med. 2010;12:260-267

43. Sarkozy A, Brugada P. Sudden cardiac death and inherited arrhythmia syndromes. J Cardiovasc Electrophysiol. 2005;16:S8-20.

44. Napolitano C, Bloise R, Monteforte N, Priori SG. Sudden cardiac death and genetic ion channelopathies: Long qt, brugada, short qt, catecholaminergic polymorphic ventricular tachycardia, and idiopathic ventricular fibrillation. Circulation. 2012;125:2027-2034

45. Clayton RH, Bernus O, Cherry EM, Dierckx H, Fenton FH, Mirabella L, Panfilov AV, Sachse FB, Seemann G, Zhang H. Models of cardiac tissue electrophysiology: Progress, challenges and open questions. Prog Biophys Mol Biol. 2011;104:22-48 
46. Piccolino M. Animal electricity and the birth of electrophysiology: The legacy of luigi galvani. Brain Res Bull. 1998;46:381-407.

47. Franz M, Schmitt C, Zrenner B, Olsson SB, Yuan S. Historical development of the monophasic action potential recording technique. Monophasic action potentials. Springer Berlin Heidelberg; 1997:3-21.

48. Camelliti P, Borg TK, Kohl P. Structural and functional characterisation of cardiac fibroblasts. Cardiovasc Res. 2005;65:40-51.

49. Streeter DD, Jr., Spotnitz HM, Patel DP, Ross J, Jr., Sonnenblick EH. Fiber orientation in the canine left ventricle during diastole and systole. Circ Res. 1969;24:339-347.

50. Lafontant RR, Feinberg H, Katz LN. Pressure-volume relationships in right ventricle. Circ Res. 1962;11:699-701.

51. Roberts WC. Pericardial heart disease: Its morphologic features and its causes. Proc (Bayl Univ Med Cent). 2005;18:38-55.

52. Suga H, Sagawa K. Instantaneous pressure-volume relationships and their ratio in the excised, supported canine left ventricle. Circ Res. 1974;35:117-126.

53. Patterson SW, Starling EH. On the mechanical factors which determine the output of the ventricles. J Physiol. 1914;48:357-379.

54. Huxley AF. Muscular contraction. J Physiol. 1974;243:1-43.

55. Bowditch H. Uber die eigenthumlichkeiten der reizbarkeit welche die muskelfasern des herzens zeigen. Arb Physiol Aust. 1871;6:139-176

56. Buckberg G, Hoffman JI, Mahajan A, Saleh S, Coghlan C. Cardiac mechanics revisited: The relationship of cardiac architecture to ventricular function. Circulation. 2008;118:25712587. doi: 2510.1161/CIRCULATIONAHA.2107.754424.

57. Robb J. The normal heart anatomy and physiology of the structural units. Am Heart J. 1942;23:455-467

58. Ho SY, Anderson RH, Sanchez-Quintana D. Atrial structure and fibres: Morphologic bases of atrial conduction. Cardiovasc Res. 2002;54:325-336.

59. Efimov IR, Nikolski VP, Rothenberg F, Greener ID, Li J, Dobrzynski H, Boyett M. Structurefunction relationship in the av junction. Anat Rec A Discov Mol Cell Evol Biol. 2004;280:952965.

60. DeWitt LM. Observations on the sino-ventricular connecting system of the mammalian heart. Anat Rec. 1909;3:475-497

61. Vassalle M. Electrogenic suppression of automaticity in sheep and dog purkinje fibers. Circ Res. 1970;27:361-377.

62. Noma A, Irisawa H, Kokobun S, Kotake H, Nishimura M, Watanabe Y. Slow current systems in the a-v node of the rabbit heart. Nature. 1980;285:228-229.

63. Vassalle M. The relationship among cardiac pacemakers. Overdrive suppression. Circ Res. 1977;41:269-277.

64. James TN. Structure and function of the sinus node, av node and his bundle of the human heart: Part ii--function. Prog Cardiovasc Dis. 2003;45:327-360.

65. Romero MF. In the beginning, there was the cell: Cellular homeostasis. Adv Physiol Educ. 2004;28:135-138.

66. Alberts B, Johnson A, Lewis J. Molecular biology of the cell. New York: Garland Science; 2002.

67. Wright SH. Generation of resting membrane potential. Adv Physiol Educ. 2004;28:139-142.

68. Girardier L, Reuben JP, Brandt PW, Grundfest H. Evidence for anion-permselective membrane in crayfish muscle fibers and its possible role in excitation-contraction coupling. $J$ Gen Physiol. 1963;47:189-214.

69. Nguyen MK, Kurtz I. Quantitative interrelationship between gibbs-donnan equilibrium, osmolality of body fluid compartments, and plasma water sodium concentration. J Appl Physiol. 2006;100:1293-1300. Epub 2005 Dec 1215. 
70. Sperelakis N. Cell physiology source book: Essentials of membrane biophysics. Elsevier/Academic Press; 2012.

71. Goldman DE. Potential, impedance, and rectification in membranes. $J$ Gen Physiol. 1943;27:37-60.

72. Hodgkin AL, Katz B. The effect of sodium ions on the electrical activity of giant axon of the squid. J Physiol. 1949;108:37-77.

73. Balser JR. The cardiac sodium channel: Gating function and molecular pharmacology. J Mol Cell Cardiol. 2001;33:599-613.

74. Bers DM. Cardiac excitation-contraction coupling. Nature. 2002;415:198-205

75. Nattel S, Carlsson L. Innovative approaches to anti-arrhythmic drug therapy. Nat Rev Drug Discov. 2006;5:1034-1049.

76. Knollmann BC, Roden DM. A genetic framework for improving arrhythmia therapy. Nature. 2008;451:929-936

77. Whalley DW, Wendt DJ, Grant AO. Basic concepts in cellular cardiac electrophysiology: Part i: Ion channels, membrane currents, and the action potential. Pacing Clin Electrophysiol. 1995;18:1556-1574.

78. Marban E, Yamagishi T, Tomaselli GF. Structure and function of voltage-gated sodium channels. J Physiol. 1998;508:647-657.

79. Reuter H. Properties of two inward membrane currents in the heart. Annu Rev Physiol. 1979;41:413-424.

80. Hibino H, Inanobe A, Furutani K, Murakami S, Findlay I, Kurachi Y. Inwardly rectifying potassium channels: Their structure, function, and physiological roles. Physiol Rev. 2010;90:291-366

81. Sigg DC, Iaizzo PA, Xiao Y-F, He B, Bakker JT, Rijen HM. Cardiac action potentials, ion channels, and gap junctions. Cardiac electrophysiology methods and models. Springer US; 2010:53-72.

82. Sanguinetti MC, Jurkiewicz NK. Two components of cardiac delayed rectifier k+ current. Differential sensitivity to block by class iii antiarrhythmic agents. J Gen Physiol. 1990;96:195215.

83. Nattel S. Delayed-rectifier potassium currents and the control of cardiac repolarization: Noble and tsien 40 years after. J Physiol. 2008;586:5849-5852. doi: 5810.1113/jphysiol.2008.163089. Epub 162008 Oct 163027.

84. Nichols CG, Makhina EN, Pearson WL, Sha Q, Lopatin AN. Inward rectification and implications for cardiac excitability. Circ Res. 1996;78:1-7.

85. Katz B. Les constantes electriques de la membrane du muscle. Arch Sci Physiol. 1949;3:285299

86. Hodgkin AL, Huxley AF. A quantitative description of membrane current and its application to conduction and excitation in nerve. J Physiol. 1952;117:500-544.

87. Stanfield PR, Nakajima S, Nakajima Y. Constitutively active and g-protein coupled inward rectifier k+ channels: Kir2.0 and kir3.0. Rev Physiol Biochem Pharmacol. 2002;145:47-179.

88. Lopatin AN, Makhina EN, Nichols CG. Potassium channel block by cytoplasmic polyamines as the mechanism of intrinsic rectification. Nature. 1994;372:366-369.

89. Ficker E, Taglialatela M, Wible BA, Henley CM, Brown AM. Spermine and spermidine as gating molecules for inward rectifier k+ channels. Science. 1994;266:1068-1072.

90. Lopatin AN, Nichols CG. Inward rectifiers in the heart: An update on i(k1). J Mol Cell Cardiol. 2001;33:625-638.

91. Armstrong CM. Sodium channels and gating currents. Physiol Rev. 1981;61:644-683.

92. Weidmann S. The effect of the cardiac membrane potential on the rapid availability of the sodium-carrying system. J Physiol. 1955;127:213-224. 
93. Noble D, Noble PJ. Late sodium current in the pathophysiology of cardiovascular disease: Consequences of sodium-calcium overload. Heart. 2006;92:iv1-iv5.

94. Sheets MF, Hanck DA, Fozzard HA. Nonlinear relation between vmax and ina in canine cardiac purkinje cells. Circ Res. 1988;63:386-398.

95. Callewaert G, Carmeliet E, Vereecke J. Single cardiac purkinje cells: General electrophysiology and voltage-clamp analysis of the pace-maker current. $J$ Physiol. 1984;349:643-661.

96. Cohen CJ, Bean BP, Tsien RW. Maximal upstroke velocity as an index of available sodium conductance. Comparison of maximal upstroke velocity and voltage clamp measurements of sodium current in rabbit purkinje fibers. Circ Res. 1984;54:636-651.

97. Dominguez G, Fozzard HA. Influence of extracellular k+ concentration on cable properties and excitability of sheep cardiac purkinje fibers. Circ Res. 1970;26:565-574.

98. Bezanilla F, Armstrong CM. Inactivation of the sodium channel. I. Sodium current experiments. J Gen Physiol. 1977;70:549-566.

99. Peon J, Ferrier GR, Moe GK. The relationship of excitability to conduction velocity in canine purkinje tissue. Circ Res. 1978;43:125-135.

100. Zipes DP, Jalife J. Cardiac electrophysiology: From cell to bedside. Saunders/Elsevier; 2009.

101. Varro A, Lathrop DA, Hester SB, Nanasi PP, Papp JG. Ionic currents and action potentials in rabbit, rat, and guinea pig ventricular myocytes. Basic Res Cardiol. 1993;88:93-102.

102. Zicha S, Moss I, Allen B, Varro A, Papp J, Dumaine R, Antzelevich C, Nattel S. Molecular basis of species-specific expression of repolarizing k+ currents in the heart. Am J Physiol Heart Circ Physiol. 2003;285:H1641-1649. Epub 2003 Jun 1619.

103. Litovsky SH, Antzelevitch C. Transient outward current prominent in canine ventricular epicardium but not endocardium. Circ Res. 1988;62:116-126.

104. Xu H, Guo W, Nerbonne JM. Four kinetically distinct depolarization-activated k+ currents in adult mouse ventricular myocytes. J Gen Physiol. 1999;113:661-678.

105. Guo W, Li H, London B, Nerbonne JM. Functional consequences of elimination of i(to,f) and i(to,s): Early afterdepolarizations, atrioventricular block, and ventricular arrhythmias in mice lacking kv1.4 and expressing a dominant-negative kv4 alpha subunit. Circ Res. 2000;87:73-79.

106. Nerbonne JM, Kass RS. Molecular physiology of cardiac repolarization. Physiol Rev. 2005;85:1205-1253.

107. Patel SP, Campbell DL. Transient outward potassium current, 'ito', phenotypes in the mammalian left ventricle: Underlying molecular, cellular and biophysical mechanisms. $J$ Physiol. 2005;569:7-39. Epub 2005 Apr 2014.

108. Beuckelmann DJ, Nabauer M, Erdmann E. Alterations of $\mathrm{k}+$ currents in isolated human ventricular myocytes from patients with terminal heart failure. Circ Res. 1993;73:379-385.

109. Zygmunt AC, Gibbons WR. Properties of the calcium-activated chloride current in heart. $J$ Gen Physiol. 1992;99:391-414.

110. Zygmunt AC, Gibbons WR. Calcium-activated chloride current in rabbit ventricular myocytes. Circ Res. 1991;68:424-437.

111. Bean BP. Two kinds of calcium channels in canine atrial cells. Differences in kinetics, selectivity, and pharmacology. J Gen Physiol. 1985;86:1-30.

112. Rudy Y. The cardiac ventricular action potential. Comprehensive physiology. John Wiley \& Sons, Inc.; 2011:531-547.

113. Bers DM. Excitation-contraction coupling and cardiac contractile force. Dordrecht, Netherlands: Kluwer Acad.; 2001.

114. Eisner DA, Lederer WJ. Na-ca exchange: Stoichiometry and electrogenicity. Am J Physiol. 1985;248:C189-202. 
115. Bers DM, Lederer WJ, Berlin JR. Intracellular ca transients in rat cardiac myocytes: Role of na-ca exchange in excitation-contraction coupling. Am J Physiol. 1990;258:C944-954.

116. Blaustein MP, Lederer WJ. Sodium/calcium exchange: Its physiological implications. Physiol Rev. 1999;79:763-854.

117. Noble D. A modification of the hodgkin--huxley equations applicable to purkinje fibre action and pace-maker potentials. J Physiol. 1962;160:317-352.

118. Attwell D, Cohen I, Eisner D, Ohba M, Ojeda C. The steady state ttx-sensitive ("window") sodium current in cardiac purkinje fibres. Pflugers Arch. 1979;379:137-142

119. Makielski JC, Farley AL. Na(+) current in human ventricle: Implications for sodium loading and homeostasis. J Cardiovasc Electrophysiol. 2006;17:S15-S20.

120. Maltsev VA, Sabbah HN, Higgins RS, Silverman N, Lesch M, Undrovinas AI. Novel, ultraslow inactivating sodium current in human ventricular cardiomyocytes. Circulation. 1998;98:2545-2552.

121. Saint DA, Ju YK, Gage PW. A persistent sodium current in rat ventricular myocytes. J Physiol. 1992;453:219-231.

122. Patlak JB, Ortiz M. Slow currents through single sodium channels of the adult rat heart. J Gen Physiol. 1985;86:89-104.

123. Ju YK, Saint DA, Gage PW. Hypoxia increases persistent sodium current in rat ventricular myocytes. J Physiol. 1996;497:337-347.

124. Undrovinas NA, Maltsev VA, Belardinelli L, Sabbah HN, Undrovinas A. Late sodium current contributes to diastolic cell ca2+ accumulation in chronic heart failure. J Physiol Sci. 2010;60:245-257

125. Kambouris NG, Nuss HB, Johns DC, Marban E, Tomaselli GF, Balser JR. A revised view of cardiac sodium channel "blockade" in the long-qt syndrome. J Clin Invest. 2000;105:11331140.

126. Levi AJ, Dalton GR, Hancox JC, Mitcheson JS, Issberner J, Bates JA, Evans SJ, Howarth FC, Hobai IA, Jones JV. Role of intracellular sodium overload in the genesis of cardiac arrhythmias. J Cardiovasc Electrophysiol. 1997;8:700-721.

127. Hammarstrom AK, Gage PW. Hypoxia and persistent sodium current. Eur Biophys J. 2002;31:323-330. Epub 2002 May 2029.

128. Clancy CE, Rudy Y. Linking a genetic defect to its cellular phenotype in a cardiac arrhythmia. Nature. 1999;400:566-569.

129. Kameyama M, Kakei M, Sato R, Shibasaki T, Matsuda H, Irisawa H. Intracellular na+ activates a k+ channel in mammalian cardiac cells. Nature. 1984;309:354-356.

130. Fozzard HA, Lipkind G. Ion channels and pumps in cardiac function. Adv Exp Med Biol. 1995;382:3-10.

131. Carmeliet E. K+ channels and control of ventricular repolarization in the heart. Fundam Clin Pharmacol. 1993;7:19-28.

132. Tohse N. Calcium-sensitive delayed rectifier potassium current in guinea pig ventricular cells. Am J Physiol. 1990;258:H1200-1207.

133. Sanguinetti MC, Bennett PB. Antiarrhythmic drug target choices and screening. Circ Res. 2003;93:491-499.

134. Liu DW, Antzelevitch C. Characteristics of the delayed rectifier current (ikr and iks) in canine ventricular epicardial, midmyocardial, and endocardial myocytes. A weaker iks contributes to the longer action potential of the m cell. Circ Res. 1995;76:351-365.

135. Bryant SM, Wan X, Shipsey SJ, Hart G. Regional differences in the delayed rectifier current (ikr and iks) contribute to the differences in action potential duration in basal left ventricular myocytes in guinea-pig. Cardiovasc Res. 1998;40:322-331.

136. Ibarra J, Morley GE, Delmar M. Dynamics of the inward rectifier k+ current during the action potential of guinea pig ventricular myocytes. Biophys J. 1991;60:1534-1539. 
137. Plaster NM, Tawil R, Tristani-Firouzi M, Canun S, Bendahhou S, Tsunoda A, Donaldson MR, Iannaccone ST, Brunt E, Barohn R, Clark J, Deymeer F, George AL, Jr., Fish FA, Hahn A, Nitu A, Ozdemir C, Serdaroglu P, Subramony SH, Wolfe G, Fu YH, Ptacek LJ. Mutations in kir2.1 cause the developmental and episodic electrical phenotypes of andersen's syndrome. Cell. 2001;105:511-519.

138. Dhamoon AS, Pandit SV, Sarmast F, Parisian KR, Guha P, Li Y, Bagwe S, Taffet SM, Anumonwo JM. Unique kir2.X properties determine regional and species differences in the cardiac inward rectifier k+ current. Circ Res. 2004;94:1332-1339. Epub 2004 Apr 1315.

139. Hume JR, Uehara A. Ionic basis of the different action potential configurations of single guinea-pig atrial and ventricular myocytes. J Physiol. 1985;368:525-544.

140. Schram G, Pourrier M, Melnyk P, Nattel S. Differential distribution of cardiac ion channel expression as a basis for regional specialization in electrical function. Circ Res. 2002;90:939950.

141. Warren M, Guha PK, Berenfeld O, Zaitsev A, Anumonwo JM, Dhamoon AS, Bagwe S, Taffet SM, Jalife J. Blockade of the inward rectifying potassium current terminates ventricular fibrillation in the guinea pig heart. J Cardiovasc Electrophysiol. 2003;14:621-631.

142. Difrancesco D. The contribution of the 'pacemaker' current (if) to generation of spontaneous activity in rabbit sino-atrial node myocytes. J Physiol. 1991;434:23-40.

143. Guo J, Ono K, Noma A. A sustained inward current activated at the diastolic potential range in rabbit sino-atrial node cells. J Physiol. 1995;483:1-13.

144. Tourneur Y. Action potential-like responses due to the inward rectifying potassium channel. J Membr Biol. 1986;90:115-122.

145. Ratan V. Handbook of human physiology. Jaypee Brothers, Medical Publishers; 2004.

146. Hille B. Ionic channels of excitable membranes. Sinauer Associates, Incorporated; 2001.

147. Lakatta EG, Maltsev VA, Vinogradova TM. A coupled system of intracellular ca2+ clocks and surface membrane voltage clocks controls the timekeeping mechanism of the heart's pacemaker. Circ Res. 2010;106:659-673

148. Korzick DH. From syncitium to regulated pump: A cardiac muscle cellular update. Adv Physiol Educ. 2011;35:22-27

149. Schmidt RF, Thews G. Cardiovascular physiology. Human physiology. New York: Springer; 2001:643.

150. Goffart S, von Kleist-Retzow JC, Wiesner RJ. Regulation of mitochondrial proliferation in the heart: Power-plant failure contributes to cardiac failure in hypertrophy. Cardiovasc Res. 2004;64:198-207.

151. Barth E, Stammler G, Speiser B, Schaper J. Ultrastructural quantitation of mitochondria and myofilaments in cardiac muscle from 10 different animal species including man. J Mol Cell Cardiol. 1992;24:669-681.

152. Sommer JR, Jennings RB. Ultrastructure of cardiac muscle. New York: Raven Press; 1992.

153. Brette F, Orchard C. T-tubule function in mammalian cardiac myocytes. Circ Res. 2003;92:1182-1192.

154. Pollack GH, Huntsman LL. Sarcomere length-active force relations in living mammalian cardiac muscle. Am J Physiol. 1974;227:383-389.

155. Fabiato A, Fabiato F. Dependence of the contractile activation of skinned cardiac cells on the sarcomere length. Nature. 1975;256:54-56.

156. Keller TC, 3rd, Eilertsen K, Higginbotham M, Kazmierski S, Kim KT, Velichkova M. Role of titin in nonmuscle and smooth muscle cells. Adv Exp Med Biol. 2000;481:265-277; discussion 278-281.

157. Maruyama K. Comparative aspects of muscle elastic proteins. Rev Physiol Biochem Pharmacol. 1999;138:1-18. 
158. Horowits R. The physiological role of titin in striated muscle. Rev Physiol Biochem Pharmacol. 1999;138:57-96.

159. Granzier $\mathrm{H}, \mathrm{Wu} \mathrm{Y}$, Siegfried L, LeWinter M. Titin: Physiological function and role in cardiomyopathy and failure. Heart Fail Rev. 2005;10:211-223.

160. Asghari P, Scriven DR, Hoskins J, Fameli N, van Breemen C, Moore ED. The structure and functioning of the couplon in the mammalian cardiomyocyte. Protoplasma. 2012;2012:0110347

161. Franzini-Armstrong C, Protasi F, Ramesh V. Shape, size, and distribution of ca(2+) release units and couplons in skeletal and cardiac muscles. Biophys J. 1999;77:1528-1539.

162. Bers DM. Calcium cycling and signaling in cardiac myocytes. Annu Rev Physiol. 2008;70:2349.

163. Soeller C, Crossman D, Gilbert R, Cannell MB. Analysis of ryanodine receptor clusters in rat and human cardiac myocytes. Proceedings of the National Academy of Sciences. 2007;104:14958-14963

164. Weiss JN, Nivala M, Garfinkel A, Qu Z. Alternans and arrhythmias: From cell to heart. Circ Res. 2011;108:98-112

165. Brandt PW, Schachat FH. Troponin c modulates the activation of thin filaments by rigor cross-bridges. Biophys J. 1997;72:2262-2267.

166. Gordon AM, Homsher E, Regnier M. Regulation of contraction in striated muscle. Physiol Rev. 2000;80:853-924.

167. McKillop DF, Geeves MA. Regulation of the interaction between actin and myosin subfragment 1: Evidence for three states of the thin filament. Biophys J. 1993;65:693-701.

168. Yue DT, Marban E, Wier WG. Relationship between force and intracellular [ca2+] in tetanized mammalian heart muscle. J Gen Physiol. 1986;87:223-242.

169. Periasamy M, Kalyanasundaram A. Serca pump isoforms: Their role in calcium transport and disease. Muscle Nerve. 2007;35:430-442.

170. Koss KL, Kranias EG. Phospholamban: A prominent regulator of myocardial contractility. Circ Res. 1996;79:1059-1063.

171. Piacentino V, 3rd, Weber CR, Chen X, Weisser-Thomas J, Margulies KB, Bers DM, Houser SR. Cellular basis of abnormal calcium transients of failing human ventricular myocytes. Circ Res. 2003;92:651-658. Epub 2003 Feb 2020.

172. Fill M, Copello JA. Ryanodine receptor calcium release channels. Physiol Rev. 2002;82:893922.

173. Hamilton SL. Ryanodine receptors. Cell Calcium. 2005;38:253-260.

174. Betzenhauser MJ, Marks AR. Ryanodine receptor channelopathies. Pflugers Arch. 2010;460:467-480

175. Terentyev D, Viatchenko-Karpinski S, Gyorke I, Volpe P, Williams SC, Gyorke S. Calsequestrin determines the functional size and stability of cardiac intracellular calcium stores: Mechanism for hereditary arrhythmia. Proc Natl Acad Sci U S A. 2003;100:11759-11764. Epub 12003 Sep 11716.

176. Postma AV, Denjoy I, Hoorntje TM, Lupoglazoff JM, Da Costa A, Sebillon P, Mannens MM, Wilde AA, Guicheney P. Absence of calsequestrin 2 causes severe forms of catecholaminergic polymorphic ventricular tachycardia. Circ Res. 2002;91:e21-26.

177. Gyorke S, Terentyev D. Modulation of ryanodine receptor by luminal calcium and accessory proteins in health and cardiac disease. Cardiovasc Res. 2008;77:245-255. Epub 2007 Oct 2015.

178. Terentyev D, Viatchenko-Karpinski S, Valdivia HH, Escobar AL, Gyorke S. Luminal ca2+ controls termination and refractory behavior of ca2+-induced ca2+ release in cardiac myocytes. Circ Res. 2002;91:414-420. 
179. Protasi F. Structural interaction between ryrs and dhprs in calcium release units of cardiac and skeletal muscle cells. Front Biosci. 2002;7:d650-658.

180. Wehrens XT, Marks A, Franzini-Armstrong C. Ryr-dhpr relationships in skeletal and cardiac muscles. Ryanodine receptors. Springer US; 2005:35-41.

181. Renganathan M, Sonntag WE, Delbono O. L-type ca2+ channel-insulin-like growth factor-1 receptor signaling impairment in aging rat skeletal muscle. Biochem Biophys Res Commun. 1997;235:784-789.

182. Meissner G, Henderson JS. Rapid calcium release from cardiac sarcoplasmic reticulum vesicles is dependent on ca2+ and is modulated by $\mathrm{mg} 2+$, adenine nucleotide, and calmodulin. J Biol Chem. 1987;262:3065-3073.

183. Balshaw DM, Xu L, Yamaguchi N, Pasek DA, Meissner G. Calmodulin binding and inhibition of cardiac muscle calcium release channel (ryanodine receptor). J Biol Chem. 2001;276:2014420153. Epub 22001 Mar 20127.

184. Pate P, Mochca-Morales J, Wu Y, Zhang JZ, Rodney GG, Serysheva, II, Williams BY, Anderson ME, Hamilton SL. Determinants for calmodulin binding on voltage-dependent ca2+ channels. J Biol Chem. 2000;275:39786-39792.

185. Brillantes AB, Ondrias K, Scott A, Kobrinsky E, Ondriasova E, Moschella MC, Jayaraman T, Landers M, Ehrlich BE, Marks AR. Stabilization of calcium release channel (ryanodine receptor) function by fk506-binding protein. Cell. 1994;77:513-523.

186. Prestle J, Janssen PM, Janssen AP, Zeitz O, Lehnart SE, Bruce L, Smith GL, Hasenfuss G. Overexpression of fk506-binding protein fkbp12.6 in cardiomyocytes reduces ryanodine receptor-mediated $\mathrm{ca}(2+)$ leak from the sarcoplasmic reticulum and increases contractility. Circ Res. 2001;88:188-194.

187. Marx SO, Reiken S, Hisamatsu Y, Jayaraman T, Burkhoff D, Rosemblit N, Marks AR. Pka phosphorylation dissociates fkbp12.6 from the calcium release channel (ryanodine receptor): Defective regulation in failing hearts. Cell. 2000;101:365-376.

188. Lewis T, Meakins J, White PD. The excitatory process in the dog's heart. Part i. The auricles. . Philosophical Transactions of the Royal Society of London. 1914;205:375-420

189. Blaustein MP, Kao JPY, Matteson DR. Cellular physiology. Elsevier/Mosby; 2004.

190. Saffitz JE, Kanter HL, Green KG, Tolley TK, Beyer EC. Tissue-specific determinants of anisotropic conduction velocity in canine atrial and ventricular myocardium. Circ Res. 1994;74:1065-1070.

191. Sheikh F, Ross RS, Chen J. Cell-cell connection to cardiac disease. Trends Cardiovasc Med. 2009;19:182-190. doi: 110.1016/j.tcm.2009.1012.1001.

192. Gutstein DE, Liu FY, Meyers MB, Choo A, Fishman GI. The organization of adherens junctions and desmosomes at the cardiac intercalated disc is independent of gap junctions. J Cell Sci. 2003;116:875-885.

193. Green KJ, Gaudry CA. Are desmosomes more than tethers for intermediate filaments? Nat Rev Mol Cell Biol. 2000;1:208-216.

194. Franke WW, Borrmann CM, Grund C, Pieperhoff S. The area composita of adhering junctions connecting heart muscle cells of vertebrates. I. Molecular definition in intercalated disks of cardiomyocytes by immunoelectron microscopy of desmosomal proteins. Eur J Cell Biol. 2006;85:69-82. Epub 2006 Jan 2009.

195. Saffitz JE. Arrhythmogenic cardiomyopathy and abnormalities of cell-to-cell coupling. Heart Rhythm. 2009;6:S62-65. doi: 10.1016/j.hrthm.2009.1003.1003. Epub 2009 Mar 1013.

196. Protonotarios N, Tsatsopoulou A. Naxos disease: Cardiocutaneous syndrome due to cell adhesion defect. Orphanet J Rare Dis. 2006;1:4.

197. Protonotarios N, Tsatsopoulou A, Patsourakos P, Alexopoulos D, Gezerlis P, Simitsis S, Scampardonis G. Cardiac abnormalities in familial palmoplantar keratosis. Br Heart J. 1986;56:321-326. 
198. Yeager M. Structure of cardiac gap junction intercellular channels. J Struct Biol. 1998;121:231-245.

199. Kumar NM, Gilula NB. The gap junction communication channel. Cell. 1996;84:381-388.

200. Saffitz JE, Deborah LL, Yamada KA. Gap junctions distribution and regulation in the heart. Cardiac electrophysiology: From cell to bedside. Elsevier Heath Sciences; 2004:181-190.

201. Saffitz JE, Yeager M. Intracardiac cell communication and gap junctions. In: Spooner PM, Rosen MR, eds. Foundation of cardiac arrhythmias. New York: Dekker; 2000:171-204.

202. Durrer D, van Dam RT, Freud GE, Janse MJ, Meijler FL, Arzbaecher RC. Total excitation of the isolated human heart. Circulation. 1970;41:899-912.

203. Kanter HL, Laing JG, Beau SL, Beyer EC, Saffitz JE. Distinct patterns of connexin expression in canine purkinje fibers and ventricular muscle. Circ Res. 1993;72:1124-1131.

204. Spach MS, Heidlage JF, Dolber PC, Barr RC. Electrophysiological effects of remodeling cardiac gap junctions and cell size: Experimental and model studies of normal cardiac growth. Circ Res. 2000;86:302-311.

205. Saffitz JE, Kleber AG. Effects of mechanical forces and mediators of hypertrophy on remodeling of gap junctions in the heart. Circ Res. 2004;94:585-591.

206. Geisler SB, Green KJ, Isom LL, Meshinchi S, Martens JR, Delmar M, Russell MW. Ordered assembly of the adhesive and electrochemical connections within newly formed intercalated disks in primary cultures of adult rat cardiomyocytes. J Biomed Biotechnol. 2010;2010:12

207. Yao JA, Gutstein DE, Liu F, Fishman GI, Wit AL. Cell coupling between ventricular myocyte pairs from connexin43-deficient murine hearts. Circ Res. 2003;93:736-743. Epub 2003 Sep 2018.

208. Pardo JV, Siliciano JD, Craig SW. A vinculin-containing cortical lattice in skeletal muscle: Transverse lattice elements ("costameres") mark sites of attachment between myofibrils and sarcolemma. Proc Natl Acad Sci U S A. 1983;80:1008-1012.

209. Danowski BA, Imanaka-Yoshida K, Sanger JM, Sanger JW. Costameres are sites of force transmission to the substratum in adult rat cardiomyocytes. The Journal of Cell Biology. 1992;118:1411-1420

210. Edman KA. Contractile performance of striated muscle. Adv Exp Med Biol. 2010;682:7-40

211. Samarel AM. Costameres, focal adhesions, and cardiomyocyte mechanotransduction. Am J Physiol Heart Circ Physiol. 2005;289:H2291-2301.

212. Takahashi K, Kakimoto Y, Toda K, Naruse K. Mechanobiology in cardiac physiology and diseases. J Cell Mol Med. 2013;17:225-232

213. Bershadsky AD, Balaban NQ, Geiger B. Adhesion-dependent cell mechanosensitivity. Annu Rev Cell Dev Biol. 2003;19:677-695.

214. Zemljic-Harpf AE, Ponrartana S, Avalos RT, Jordan MC, Roos KP, Dalton ND, Phan VQ, Adamson ED, Ross RS. Heterozygous inactivation of the vinculin gene predisposes to stressinduced cardiomyopathy. Am J Pathol. 2004;165:1033-1044.

215. Shai SY, Harpf AE, Ross RS. Integrins and the myocardium. Genet Eng (N Y). 2002;24:87-105.

216. Tinsley JM, Blake DJ, Zuellig RA, Davies KE. Increasing complexity of the dystrophinassociated protein complex. Proc Natl Acad Sci U S A. 1994;91:8307-8313.

217. Albrecht DE, Froehner SC. Syntrophins and dystrobrevins: Defining the dystrophin scaffold at synapses. Neurosignals. 2002;11:123-129.

218. Le Rumeur E, Winder SJ, Hubert JF. Dystrophin: More than just the sum of its parts. Biochim Biophys Acta. 2010;2010:1713-1722

219. Hoffman EP, Brown RH, Jr., Kunkel LM. Dystrophin: The protein product of the duchenne muscular dystrophy locus. Cell. 1987;51:919-928

220. Lapidos KA, Kakkar R, McNally EM. The dystrophin glycoprotein complex: Signaling strength and integrity for the sarcolemma. Circ Res. 2004;94:1023-1031. 
221. Blake DJ, Weir A, Newey SE, Davies KE. Function and genetics of dystrophin and dystrophinrelated proteins in muscle. Physiol Rev. 2002;82:291-329.

222. Barnea E, Zuk D, Simantov R, Nudel U, Yaffe D. Specificity of expression of the muscle and brain dystrophin gene promoters in muscle and brain cells. Neuron. 1990;5:881-888.

223. Emery A. Duchenne muscular dystrophy. Oxford: Oxford University Press; 1993.

224. Nowak KJ, Davies KE. Duchenne muscular dystrophy and dystrophin: Pathogenesis and opportunities for treatment. EMBO Rep. 2004;5:872-876.

225. Klingler W, Jurkat-Rott K, Lehmann-Horn F, Schleip R. The role of fibrosis in duchenne muscular dystrophy. Acta Myol. 2012;31:184-195.

226. Gardner-Medwin D. Mutation rate in duchenne type of muscular dystrophy. J Med Genet. 1970;7:334-337.

227. Banks GB, Chamberlain JS. The value of mammalian models for duchenne muscular dystrophy in developing therapeutic strategies. Curr Top Dev Biol. 2008;84:43153.:10.1016/S0070-2153(1008)00609-00601.

228. Whitehead NP, Yeung EW, Allen DG. Muscle damage in mdx (dystrophic) mice: Role of calcium and reactive oxygen species. Clin Exp Pharmacol Physiol. 2006;33:657-662.

229. Milan DJ, MacRae CA. Animal models for arrhythmias. Cardiovasc Res. 2005;67:426-437

230. Beastrom N, Lu H, Macke A, Canan BD, Johnson EK, Penton CM, Kaspar BK, Rodino-Klapac LR, Zhou L, Janssen PM, Montanaro F. Mdx((5)cv) mice manifest more severe muscle dysfunction and diaphragm force deficits than do mdx mice. Am J Pathol. 2011;179:24642474

231. Deconinck N, Dan B. Pathophysiology of duchenne muscular dystrophy: Current hypotheses. Pediatr Neurol. 2007;36:1-7.

232. Allen DG, Whitehead NP. Duchenne muscular dystrophy--what causes the increased membrane permeability in skeletal muscle? Int J Biochem Cell Biol. 2011;43:290-294

233. Bradley WG, Fulthorpe JJ. Studies of sarcolemmal integrity in myopathic muscle. Neurology. 1978;28:670-677

234. Gumerson JD, Michele DE. The dystrophin-glycoprotein complex in the prevention of muscle damage. J Biomed Biotechnol. 2011;2011:5

235. Townsend D, Yasuda S, McNally E, Metzger JM. Distinct pathophysiological mechanisms of cardiomyopathy in hearts lacking dystrophin or the sarcoglycan complex. Faseb J. 2011;25:3106-3114

236. Gee SH, Madhavan R, Levinson SR, Caldwell JH, Sealock R, Froehner SC. Interaction of muscle and brain sodium channels with multiple members of the syntrophin family of dystrophinassociated proteins. J Neurosci. 1998;18:128-137.

237. Gavillet B, Rougier JS, Domenighetti AA, Behar R, Boixel C, Ruchat P, Lehr HA, Pedrazzini T, Abriel H. Cardiac sodium channel nav1.5 is regulated by a multiprotein complex composed of syntrophins and dystrophin. Circ Res. 2006;99:407-414. Epub 2006 Jul 2020.

238. Petitprez S, Zmoos AF, Ogrodnik J, Balse E, Raad N, El-Haou S, Albesa M, Bittihn P, Luther S, Lehnart SE, Hatem SN, Coulombe A, Abriel H. Sap97 and dystrophin macromolecular complexes determine two pools of cardiac sodium channels nav1.5 in cardiomyocytes. Circ Res. 2011;108:294-304

239. El-Haou S, Balse E, Neyroud N, Dilanian G, Gavillet B, Abriel H, Coulombe A, Jeromin A, Hatem SN. Kv4 potassium channels form a tripartite complex with the anchoring protein sap97 and camkii in cardiac myocytes. Circ Res. 2009;104:758-769. doi: 710.1161/CIRCRESAHA.1108.191007. Epub 192009 Feb 191012.

240. Abi-Char J, El-Haou S, Balse E, Neyroud N, Vranckx R, Coulombe A, Hatem SN. The anchoring protein sap97 retains kv1.5 channels in the plasma membrane of cardiac myocytes. $\mathrm{Am} \mathrm{J}$ 
Physiol Heart Circ Physiol. 2008;294:H1851-1861. doi: 1810.1152/ajpheart.01045.02007. Epub 02008 Feb 01041.

241. Vikstrom KL, Vaidyanathan R, Levinsohn S, O'Connell RP, Qian Y, Crye M, Mills JH, Anumonwo JM. Sap97 regulates kir2.3 channels by multiple mechanisms. Am J Physiol Heart Circ Physiol. 2009;297:H1387-1397

242. Cohen SA. Immunocytochemical localization of rh1 sodium channel in adult rat heart atria and ventricle. Presence in terminal intercalated disks. Circulation. 1996;94:3083-3086

243. Maier SK, Westenbroek RE, Schenkman KA, Feigl EO, Scheuer T, Catterall WA. An unexpected role for brain-type sodium channels in coupling of cell surface depolarization to contraction in the heart. Proc Natl Acad Sci U S A. 2002;99:4073-4078

244. Kucera JP, Rohr S, Rudy Y. Localization of sodium channels in intercalated disks modulates cardiac conduction. Circ Res. 2002;91:1176-1182

245. Haimovich B, Bonilla E, Casadei J, Barchi R. Immunocytochemical localization of the mammalian voltage-dependent sodium channel using polyclonal antibodies against the purified protein. The Journal of neuroscience : the official journal of the Society for Neuroscience. 1984;4:2259-2268

246. Smaill BH, Zhao J, Trew ML. Three-dimensional impulse propagation in myocardium: Arrhythmogenic mechanisms at the tissue level. Circ Res. 2013;112:834-848

247. Helm PA, Tseng HJ, Younes L, McVeigh ER, Winslow RL. Ex vivo 3d diffusion tensor imaging and quantification of cardiac laminar structure. Magnetic resonance in medicine : official journal of the Society of Magnetic Resonance in Medicine / Society of Magnetic Resonance in Medicine. 2005;54:850-859

248. Gilbert SH, Benoist D, Benson AP, White E, Tanner SF, Holden AV, Dobrzynski H, Bernus O, Radjenovic A. Visualization and quantification of whole rat heart laminar structure using high-spatial resolution contrast-enhanced mri. Am J Physiol Heart Circ Physiol. 2012;302:H287-298

249. Sakuma H. Magnetic resonance imaging for ischemic heart disease. Journal of magnetic resonance imaging : JMRI. 2007;26:3-13

250. Schwab BC, Seemann G, Lasher RA, Torres NS, Wulfers EM, Arp M, Carruth ED, Bridge JH, Sachse FB. Quantitative analysis of cardiac tissue including fibroblasts using threedimensional confocal microscopy and image reconstruction: Towards a basis for electrophysiological modeling. IEEE transactions on medical imaging. 2013;32:862-872

251. Spach M. The discontinuous nature of electrical propagation in cardiac muscle. Consideration of a quantitative model incorporating the membrane ionic properties and structural complexities. Annals of Biomedical Engineering. 1983;11:209-261

252. Lieberman M, Kootsey JM, Johnson EA, Sawanobori T. Low conduction in cardiac muscle. Biophysical model. Biophys J. 1973;13:37-55

253. Sharp GH, Joyner RW. Simulated propagation of cardiac action potentials. Biophys J. 1980;31:403-423

254. Kleber AG, Rudy Y. Basic mechanisms of cardiac impulse propagation and associated arrhythmias. Physiol Rev. 2004;84:431-488

255. Luo CH, Rudy Y. A dynamic model of the cardiac ventricular action potential. Ii. Afterdepolarizations, triggered activity, and potentiation. Circ Res. 1994;74:1097-1113.

256. Joyner RW. Effects of the discrete pattern of electrical coupling on propagation through an electrical syncytium. Circ Res. 1982;50:192-200.

257. Rudy Y. Electrotonic cell-cell interactions in cardiac tissue: Effects on action potential propagation and repolarization. Ann. N.Y. Acad. Sci. 2005;1047:308-313

258. Vogel R, Weingart R. Mathematical model of vertebrate gap junctions derived from electrical measurements on homotypic and heterotypic channels. J Physiol. 1998;510 ( Pt 1):177-189 
259. Armstrong CM. Na channel inactivation from open and closed states. Proc Natl Acad Sci U S A. 2006;103:17991-17996. Epub 12006 Nov 17913.

260. Abriel H. Cardiac sodium channel nav1.5 and its associated proteins. Arch Mal Coeur Vaiss. 2007;100:787-793.

261. Antzelevitch C, Yan GX, Shimizu W. Transmural dispersion of repolarization and arrhythmogenicity: The brugada syndrome versus the long qt syndrome. J Electrocardiol. 1999;32:158-165.

262. Antzelevitch C, Fish J. Electrical heterogeneity within the ventricular wall. Basic Res Cardiol. 2001;96:517-527.

263. Akar FG, Yan GX, Antzelevitch C, Rosenbaum DS. Unique topographical distribution of m cells underlies reentrant mechanism of torsade de pointes in the long-qt syndrome. Circulation. 2002;105:1247-1253.

264. Antzelevitch C. Role of spatial dispersion of repolarization in inherited and acquired sudden cardiac death syndromes. Am J Physiol Heart Circ Physiol. 2007;293:H2024-2038

265. Antzelevitch C, Sicouri S, Litovsky SH, Lukas A, Krishnan SC, Di Diego JM, Gintant GA, Liu DW. Heterogeneity within the ventricular wall. Electrophysiology and pharmacology of epicardial, endocardial, and m cells. Circ Res. 1991;69:1427-1449

266. Nabauer M. Electrical heterogeneity in the ventricular wall--and the m cell. Cardiovasc Res. 1998;40:248-250

267. Litovsky SH, Antzelevitch C. Rate dependence of action potential duration and refractoriness in canine ventricular endocardium differs from that of epicardium: Role of the transient outward current. J Am Coll Cardiol. 1989;14:1053-1066.

268. Anyukhovsky EP, Sosunov EA, Rosen MR. Regional differences in electrophysiological properties of epicardium, midmyocardium, and endocardium. In vitro and in vivo correlations. Circulation. 1996;94:1981-1988

269. Sicouri S, Antzelevitch C. A subpopulation of cells with unique electrophysiological properties in the deep subepicardium of the canine ventricle. The $\mathrm{m}$ cell. Circ Res. 1991;68:1729-1741

270. Viswanathan PC, Shaw RM, Rudy Y. Effects of ikr and iks heterogeneity on action potential duration and its rate dependence: A simulation study. Circulation. 1999;99:2466-2474

271. Akar FG. Left ventricular repolarization heterogeneity as an arrhythmic substrate in heart failure. Minerva. 2010;58:205-212.

272. Taggart P, Sutton PM, Spear DW, Drake HF, Swanton RH, Emanuel RW. Simultaneous endocardial and epicardial monophasic action potential recordings during brief periods of coronary artery ligation in the dog: Influence of adrenaline, beta blockade and alpha blockade. Cardiovasc Res. 1988;22:900-909

273. Gilmour RF, Jr., Zipes DP. Different electrophysiological responses of canine endocardium and epicardium to combined hyperkalemia, hypoxia, and acidosis. Circ Res. 1980;46:814825

274. Tanawuttiwat T, Harindhanavudhi T, Bhan A, Dia M. Hyperkalemia-induced brugada pattern: An unusual manifestation. Journal of cardiovascular medicine (Hagerstown, Md.). 2010;11:285-287

275. Littmann L, Monroe MH, Taylor L, 3rd, Brearley WD, Jr. The hyperkalemic brugada sign. Journal of electrocardiology. 2007;40:53-59

276. Naccarelli GV, Antzelevitch C, Wolbrette DL, Luck JC. The brugada syndrome. Curr Opin Cardiol. 2002;17:19-23

277. Muthappan P, Calkins H. Arrhythmogenic right ventricular dysplasia. Prog Cardiovasc Dis. 2008;51:31-43. doi: 10.1016/j.pcad.2008.1001.1002.

278. Haugaa KH, Leren IS, Berge KE, Bathen J, Loennechen JP, Anfinsen OG, Fruh A, Edvardsen T, Kongsgard E, Leren TP, Amlie JP. High prevalence of exercise-induced arrhythmias in 
catecholaminergic polymorphic ventricular tachycardia mutation-positive family members diagnosed by cascade genetic screening. Europace. 2010;12:417-423

279. Antzelevitch C, Shimizu W. Cellular mechanisms underlying the long qt syndrome. Curr Opin Cardiol. 2002;17:43-51

280. Dumaine R, Antzelevitch C. Molecular mechanisms underlying the long qt syndrome. Curr Opin Cardiol. 2002;17:36-42

281. Shimizu W, Antzelevitch C. Effects of a $\mathrm{k}(+)$ channel opener to reduce transmural dispersion of repolarization and prevent torsade de pointes in lqt1, lqt2, and lqt3 models of the long-qt syndrome. Circulation. 2000;102:706-712

282. Shimizu W, Antzelevitch C. Sodium channel block with mexiletine is effective in reducing dispersion of repolarization and preventing torsade des pointes in lqt 2 and lqt 3 models of the long-qt syndrome. Circulation. 1997;96:2038-2047.

283. Glukhov AV, Fedorov VV, Kalish PW, Ravikumar VK, Lou Q, Janks D, Schuessler RB, Moazami $\mathrm{N}$, Efimov IR. Conduction remodeling in human end-stage nonischemic left ventricular cardiomyopathy. Circulation. 2012;125:1835-1847

284. Morley GE, Vaidya D, Samie FH, Lo C, Delmar M, Jalife J. Characterization of conduction in the ventricles of normal and heterozygous cx43 knockout mice using optical mapping. $J$ Cardiovasc Electrophysiol. 1999;10:1361-1375.

285. van Rijen HV, Eckardt D, Degen J, Theis M, Ott T, Willecke K, Jongsma HJ, Opthof T, de Bakker JM. Slow conduction and enhanced anisotropy increase the propensity for ventricular tachyarrhythmias in adult mice with induced deletion of connexin43. Circulation. 2004;109:1048-1055

286. Stein M, van Veen TA, Hauer RN, de Bakker JM, van Rijen HV. A 50\% reduction of excitability but not of intercellular coupling affects conduction velocity restitution and activation delay in the mouse heart. PloS one. 2011;6:e20310

287. Danik SB, Liu F, Zhang J, Suk HJ, Morley GE, Fishman GI, Gutstein DE. Modulation of cardiac gap junction expression and arrhythmic susceptibility. Circ Res. 2004;95:1035-1041

288. Severs NJ, Bruce AF, Dupont E, Rothery S. Remodelling of gap junctions and connexin expression in diseased myocardium. Cardiovasc Res. 2008;80:9-19

289. Tomaselli GF, Zipes DP. What causes sudden death in heart failure? Circ Res. 2004;95:754763.

290. Tomaselli GF, Marban E. Electrophysiological remodeling in hypertrophy and heart failure. Cardiovasc Res. 1999;42:270-283.

291. Akar FG, Rosenbaum DS. Transmural electrophysiological heterogeneities underlying arrhythmogenesis in heart failure. Circ Res. 2003;93:638-645

292. Akar JG, Akar FG. Mapping arrhythmias in the failing heart: From langendorff to patient. $J$ Electrocardiol. 2006;39:S19-23. Epub 2006 Aug 2021.

293. Hoffman BF, Cranefield PF. Electrophysiology of the heart. John Wiley \& Sons, Incorporated; 1976.

294. Cranefield PF. Action potentials, afterdepolarizations, and arrhythmias. Circ Res. 1977;41:415-423

295. Cranefield PF. The conduction of the cardiac impulse. New York: Futura Press; 1975.

296. Zipes DP, Foster PR, Troup PJ, Pedersen DH. Atrial induction of ventricular tachycardia: Reentry versus triggered automaticity. Am J Cardiol. 1979;44:1-8.

297. Brachmann J, Scherlag BJ, Rosenshtraukh LV, Lazzara R. Bradycardia-dependent triggered activity: Relevance to drug-induced multiform ventricular tachycardia. Circulation. 1983;68:846-856.

298. Ferrier GR. Digitalis arrhythmias: Role of oscillatory afterpotentials. Prog Cardiovasc Dis. 1977;19:459-474. 
299. Xie Y, Sato D, Garfinkel A, Qu Z, Weiss JN. So little source, so much sink: Requirements for afterdepolarizations to propagate in tissue. Biophys J. 2010;99:1408-1415

300. Rosenbaum MB, Elizari MV, Brugada P, Wellens HJJ. The role of triggered activity in clinical arrhythmias. Frontiers of cardiac electrophysiology. Springer Netherlands; 1983:195-216.

301. Gavrilescu S, Luca C. Right ventricular monophasic action potentials in patients with long qt syndrome. Br Heart J. 1978;40:1014-1018.

302. Gadsby DC, Cranefield PF. Two levels of resting potential in cardiac purkinje fibers. J Gen Physiol. 1977;70:725-746.

303. Carmeliet EE. Chloride ions and the membrane potential of purkinje fibres. $J$ Physiol. 1961;156:375-388.

304. Coraboeuf E, Deroubaix E, Coulombe A. Acidosis-induced abnormal repolarization and repetitive activity in isolated dog purkinje fibers. J Physiol (Paris). 1980;76:97-106.

305. Sano T, Sawanobori T. Abnormal automaticity in canine purkinje fibers focally subjected to low external concentrations of calcium. Circ Res. 1972;31:158-164.

306. Trautwein W, Gottstein U, Dudel J. [the action current of the myocardial fibers in oxygen deficiency]. Pflugers Arch. 1954;260:40-60.

307. Lazzara R. Antiarrhythmic drugs and torsade de pointes. Eur Heart J. 1993;14:88-92.

308. Weiss JN, Garfinkel A, Karagueuzian HS, Chen PS, Qu Z. Early afterdepolarizations and cardiac arrhythmias. Heart Rhythm. 2010;7:1891-1899

309. January CT, Riddle JM. Early afterdepolarizations: Mechanism of induction and block. A role for l-type ca2+ current. Circ Res. 1989;64:977-990.

310. Rosen MR. The concept of afterdepolarization. In: Rosen MR, Janse MJ, Wit AL, eds. Cardiac electrophysiology: A textbook. New York: Futura Publishing Co Inc.; 1990:267-282.

311. Rosen MR, Danilo PJ. Digitalis-induced delayed afterdepolarizations. In: Zipes DP, Bailey JC, Elharrar V, eds. The slow inward current and cardiac arrhythmias. Boston: Martinus Nijhoff Publishers; 1980:417-435.

312. Ferrier GR, Moe GK. Effect of calcium on acetylstrophanthidin-induced transient depolarizations in canine purkinje tissue. Circ Res. 1973;33:508-515.

313. Hoffman BF, Rosen MR. Cellular mechanisms for cardiac arrhythmias. Circ Res. 1981;49:115.

314. Volders PG, Kulcsar A, Vos MA, Sipido KR, Wellens HJ, Lazzara R, Szabo B. Similarities between early and delayed afterdepolarizations induced by isoproterenol in canine ventricular myocytes. Cardiovasc Res. 1997;34:348-359.

315. Buchanan JW, Jr., Saito T, Gettes LS. The effects of antiarrhythmic drugs, stimulation frequency, and potassium-induced resting membrane potential changes on conduction velocity and dv/dtmax in guinea pig myocardium. Circ Res. 1985;56:696-703

316. Arnsdorf MF. The cellular basis of cardiac arrhythmias. A matrical perspective. Ann N Y Acad Sci. 1990;601:263-280.

317. Cranefield PF, Klein HO, Hoffman BF. Conduction of the cardiac impulse. 1. Delay, block, and one-way block in depressed purkinje fibers. Circ Res. 1971;28:199-219.

318. Cranefield PF, Wit AL, Hoffman BF. Conduction of the cardiac impulse. 3. Characteristics of very slow conduction. J Gen Physiol. 1972;59:227-246.

319. Spach MS, Miller WT, 3rd, Geselowitz DB, Barr RC, Kootsey JM, Johnson EA. The discontinuous nature of propagation in normal canine cardiac muscle. Evidence for recurrent discontinuities of intracellular resistance that affect the membrane currents. Circ Res. 1981;48:39-54

320. Rosen MR. The links between basic and clinical cardiac electrophysiology. Circulation. 1988;77:251-263

321. Clerc L. Directional differences of impulse spread in trabecular muscle from mammalian heart. J Physiol. 1976;255:335-346 
322. Wit AL, Hoffman BF, Cranefield PF. Slow conduction and reentry in the ventricular conducting system. I. Return extrasystole in canine purkinje fibers. Circ Res. 1972;30:1-10.

323. Makielski JC, Sheets MF, Hanck DA, January CT, Fozzard HA. Sodium current in voltage clamped internally perfused canine cardiac purkinje cells. Biophys J. 1987;52:1-11.

324. Rose WC, Balke CW, Wier WG, Marban E. Macroscopic and unitary properties of physiological ion flux through l-type ca2+ channels in guinea-pig heart cells. $J$ Physiol. 1992;456:267-284.

325. Catterall WA, Goldin AL, Waxman SG. Nomenclature and structure-function relationships of voltage-gated sodium channels. IUPHAR Compendium of Voltage-Gated Ion Channels. 2005;57:397-409

326. Catterall WA. From ionic currents to molecular mechanisms: The structure and function of voltage-gated sodium channels. Neuron. 2000;26:13-25.

327. Fozzard HA, Hanck DA. Structure and function of voltage-dependent sodium channels: Comparison of brain ii and cardiac isoforms. Physiol Rev. 1996;76:887-926.

328. Goldin AL, Barchi RL, Caldwell JH, Hofmann F, Howe JR, Hunter JC, Kallen RG, Mandel G, Meisler MH, Netter YB, Noda M, Tamkun MM, Waxman SG, Wood JN, Catterall WA. Nomenclature of voltage-gated sodium channels. Neuron. 2000;28:365-368.

329. Plummer NW, Meisler MH. Evolution and diversity of mammalian sodium channel genes. Genomics. 1999;57:323-331.

330. Lehmann-Horn F, Jurkat-Rott K. Voltage-gated ion channels and hereditary disease. Physiol Rev. 1999;79:1317-1372.

331. Armstrong CM, Bezanilla F. Inactivation of the sodium channel. Ii. Gating current experiments. J Gen Physiol. 1977;70:567-590.

332. Keynes RD. The kinetics of voltage-gated ion channels. Q Rev Biophys. 1994;27:339-434.

333. Aldrich RW, Corey DP, Stevens CF. A reinterpretation of mammalian sodium channel gating based on single channel recording. Nature. 1983;306:436-441.

334. Krogh-Madsen T, Christini DJ. Nonlinear dynamics in cardiology. Annu Rev Biomed Eng. 2012;14:179-203

335. Catterall WA, Raman IM, Robinson HP, Sejnowski TJ, Paulsen O. The hodgkin-huxley heritage: From channels to circuits. The Journal of neuroscience : the official journal of the Society for Neuroscience. 2012;32:14064-14073

336. Hartshorne RP, Messner DJ, Coppersmith JC, Catterall WA. The saxitoxin receptor of the sodium channel from rat brain. Evidence for two nonidentical beta subunits. J Biol Chem. 1982;257:13888-13891.

337. Yu FH, Westenbroek RE, Silos-Santiago I, McCormick KA, Lawson D, Ge P, Ferriera H, Lilly J, DiStefano PS, Catterall WA, Scheuer T, Curtis R. Sodium channel beta4, a new disulfidelinked auxiliary subunit with similarity to beta2.J Neurosci. 2003;23:7577-7585.

338. Sills MN, Xu YC, Baracchini E, Goodman RH, Cooperman SS, Mandel G, Chien KR. Expression of diverse na+ channel messenger rnas in rat myocardium. Evidence for a cardiac-specific na+ channel. J Clin Invest. 1989;84:331-336.

339. Rogart RB, Cribbs LL, Muglia LK, Kephart DD, Kaiser MW. Molecular cloning of a putative tetrodotoxin-resistant rat heart nat channel isoform. Proc Natl Acad Sci U $S$ A. 1989;86:8170-8174.

340. Zimmer T, Bollensdorff C, Haufe V, Birch-Hirschfeld E, Benndorf K. Mouse heart na+ channels: Primary structure and function of two isoforms and alternatively spliced variants. Am J Physiol Heart Circ Physiol. 2002;282:H1007-1017.

341. Coraboeuf E, Deroubaix E, Coulombe A. Effect of tetrodotoxin on action potentials of the conducting system in the dog heart. Am J Physiol. 1979;236:H561-567. 
342. Noda M, Shimizu S, Tanabe T, Takai T, Kayano T, Ikeda T, Takahashi H, Nakayama H, Kanaoka Y, Minamino N, et al. Primary structure of electrophorus electricus sodium channel deduced from cdna sequence. Nature. 1984;312:121-127.

343. Catterall WA. Molecular properties of voltage-sensitive sodium channels. Annu Rev Biochem. 1986;55:953-985.

344. Ritchie JM. Sodium-channel turnover in rabbit cultured schwann cells. Proc $R$ Soc Lond B Biol Sci. 1988;233:423-430.

345. James WM, Agnew WS. Alpha-(2----8)-polysialic acid immunoreactivity in voltage-sensitive sodium channel of eel electric organ. Proc R Soc Lond B Biol Sci. 1989;237:233-245.

346. Cohen SA, Levitt LK. Partial characterization of the rh1 sodium channel protein from rat heart using subtype-specific antibodies. Circ Res. 1993;73:735-742.

347. Noda M, Suzuki H, Numa S, Stuhmer W. A single point mutation confers tetrodotoxin and saxitoxin insensitivity on the sodium channel ii. FEBS Lett. 1989;259:213-216.

348. Benitah JP, Ranjan R, Yamagishi T, Janecki M, Tomaselli GF, Marban E. Molecular motions within the pore of voltage-dependent sodium channels. Biophys J. 1997;73:603-613.

349. McPhee JC, Ragsdale DS, Scheuer T, Catterall WA. A mutation in segment ivs6 disrupts fast inactivation of sodium channels. Proc Natl Acad Sci U S A. 1994;91:12346-12350.

350. Holmgren M, Smith PL, Yellen G. Trapping of organic blockers by closing of voltagedependent k+ channels: Evidence for a trap door mechanism of activation gating. $J$ Gen Physiol. 1997;109:527-535.

351. Perez-Garcia MT, Chiamvimonvat N, Ranjan R, Balser JR, Tomaselli GF, Marban E. Mechanisms of sodium/calcium selectivity in sodium channels probed by cysteine mutagenesis and sulfhydryl modification. Biophys J. 1997;72:989-996.

352. Heinemann SH, Terlau H, Stuhmer W, Imoto K, Numa S. Calcium channel characteristics conferred on the sodium channel by single mutations. Nature. 1992;356:441-443.

353. Stuhmer W, Conti F, Suzuki H, Wang XD, Noda M, Yahagi N, Kubo H, Numa S. Structural parts involved in activation and inactivation of the sodium channel. Nature. 1989;339:597-603.

354. Kontis KJ, Goldin AL. Sodium channel inactivation is altered by substitution of voltage sensor positive charges. J Gen Physiol. 1997;110:403-413.

355. Yang N, Horn R. Evidence for voltage-dependent s4 movement in sodium channels. Neuron. 1995;15:213-218.

356. Kontis KJ, Rounaghi A, Goldin AL. Sodium channel activation gating is affected by substitutions of voltage sensor positive charges in all four domains. J Gen Physiol. 1997;110:391-401.

357. Yang N, George AL, Jr., Horn R. Molecular basis of charge movement in voltage-gated sodium channels. Neuron. 1996;16:113-122.

358. Chen LQ, Chahine M, Kallen RG, Barchi RL, Horn R. Chimeric study of sodium channels from rat skeletal and cardiac muscle. FEBS Lett. 1992;309:253-257.

359. Satin J, Kyle JW, Chen M, Bell P, Cribbs LL, Fozzard HA, Rogart RB. A mutant of ttx-resistant cardiac sodium channels with ttx-sensitive properties. Science. 1992;256:1202-1205.

360. Catterall WA. Molecular properties of brain sodium channels: An important target for anticonvulsant drugs. Adv Neurol. 1999;79:441-456.

361. Hille B. Local anesthetics: Hydrophilic and hydrophobic pathways for the drug-receptor reaction. The Journal of general physiology. 1977;69:497-515

362. Hondeghem LM, Katzung BG. Time- and voltage-dependent interactions of antiarrhythmic drugs with cardiac sodium channels. Biochim Biophys Acta. 1977;472:373-398.

363. Rivolta I, Clancy CE, Tateyama M, Liu H, Priori SG, Kass RS. A novel scn5a mutation associated with long qt-3: Altered inactivation kinetics and channel dysfunction. Physiol Genomics. 2002;10:191-197. 
364. Armstrong CM, Bezanilla F, Rojas E. Destruction of sodium conductance inactivation in squid axons perfused with pronase. J Gen Physiol. 1973;62:375-391.

365. West JW, Patton DE, Scheuer T, Wang Y, Goldin AL, Catterall WA. A cluster of hydrophobic amino acid residues required for fast na(+)-channel inactivation. Proc Natl Acad Sci U S A. 1992;89:10910-10914.

366. Kubota T, Kinoshita M, Sasaki R, Aoike F, Takahashi MP, Sakoda S, Hirose K. New mutation of the na channel in the severe form of potassium-aggravated myotonia. Muscle Nerve. 2009;39:666-673. doi: 610.1002/mus.21155.

367. Cannon SC. Slow inactivation of sodium channels: More than just a laboratory curiosity. Biophys J. 1996;71:5-7.

368. Cummins TR, Sigworth FJ. Impaired slow inactivation in mutant sodium channels. Biophys J. 1996;71:227-236.

369. Vilin YY, Makita N, George AL, Jr., Ruben PC. Structural determinants of slow inactivation in human cardiac and skeletal muscle sodium channels. Biophys J. 1999;77:1384-1393.

370. Veldkamp MW, Viswanathan PC, Bezzina C, Baartscheer A, Wilde AA, Balser JR. Two distinct congenital arrhythmias evoked by a multidysfunctional na(+) channel. Circ Res. 2000;86:E91-97.

371. Isom LL, De Jongh KS, Patton DE, Reber BF, Offord J, Charbonneau H, Walsh K, Goldin AL, Catterall WA. Primary structure and functional expression of the beta 1 subunit of the rat brain sodium channel. Science. 1992;256:839-842.

372. Isom LL, Ragsdale DS, De Jongh KS, Westenbroek RE, Reber BF, Scheuer T, Catterall WA. Structure and function of the beta 2 subunit of brain sodium channels, a transmembrane glycoprotein with a cam motif. Cell. 1995;83:433-442.

373. Abriel H, Kass RS. Regulation of the voltage-gated cardiac sodium channel nav1.5 by interacting proteins. Trends Cardiovasc Med. 2005;15:35-40.

374. Markovich D. Expression cloning of membrane proteins in xenopus oocytes. Nat Protocol Exchange. 2007

375. Medeiros-Domingo A, Kaku T, Tester DJ, Iturralde-Torres P, Itty A, Ye B, Valdivia C, Ueda K, Canizales-Quinteros S, Tusie-Luna MT, Makielski JC, Ackerman MJ. Scn4b-encoded sodium channel beta4 subunit in congenital long-qt syndrome. Circulation. 2007;116:134-142. Epub 2007 Jun 2025.

376. Watanabe H, Koopmann TT, Le Scouarnec S, Yang T, Ingram CR, Schott JJ, Demolombe S, Probst V, Anselme F, Escande D, Wiesfeld AC, Pfeufer A, Kaab S, Wichmann HE, Hasdemir C, Aizawa Y, Wilde AA, Roden DM, Bezzina CR. Sodium channel beta1 subunit mutations associated with brugada syndrome and cardiac conduction disease in humans. J Clin Invest. 2008;118:2260-2268. doi: 2210.1172/JCI33891.

377. Wallace RH, Wang DW, Singh R, Scheffer IE, George AL, Jr., Phillips HA, Saar K, Reis A, Johnson EW, Sutherland GR, Berkovic SF, Mulley JC. Febrile seizures and generalized epilepsy associated with a mutation in the na+-channel beta1 subunit gene scn1b. Nat Genet. 1998;19:366-370.

378. Xu R, Thomas EA, Gazina EV, Richards KL, Quick M, Wallace RH, Harkin LA, Heron SE, Berkovic SF, Scheffer IE, Mulley JC, Petrou S. Generalized epilepsy with febrile seizures plusassociated sodium channel beta1 subunit mutations severely reduce beta subunit-mediated modulation of sodium channel function. Neuroscience. 2007;148:164-174. Epub $2007 \mathrm{Jul}$ 2012.

379. Noebels JL. Sodium channel gene expression and epilepsy. Novartis Found Symp. 2002;241:109-120; discussion 120-103, 226-132.

380. Duff HJ, Offord J, West J, Catterall WA. Class i and iv antiarrhythmic drugs and cytosolic calcium regulate mrna encoding the sodium channel alpha subunit in rat cardiac muscle. $\mathrm{Mol}$ Pharmacol. 1992;42:570-574. 
381. Priori SG. Inherited arrhythmogenic diseases: The complexity beyond monogenic disorders. Circ Res. 2004;94:140-145.

382. Antzelevich C, Yan GX, Shimuzu W, Burashnikov A. Electrical heterogeneity, the ecg and cardiac arrhythmias. In: Zipes DP, Jalife J, eds. Cardiac electrophysiology: From cell to bedside. Philadelphia: Saunders; 2000:222-238.

383. Kumar A, Cannon CP. Acute coronary syndromes: Diagnosis and management, part i. Mayo Clin Proc. 2009;84:917-938. doi: 910.1016/S0025-6196(1011)60509-60500.

384. Sarkozy A, Brugada P. Sudden cardiac death: What is inside our genes? Can J Cardiol. 2005;21:1099-1110.

385. Clancy CE, Kass RS. Inherited and acquired vulnerability to ventricular arrhythmias: Cardiac na+ and k+ channels. Physiol Rev. 2005;85:33-47.

386. Clancy CE, Kurokawa J, Tateyama M, Wehrens XH, Kass RS. K+ channel structure-activity relationships and mechanisms of drug-induced qt prolongation. Annu Rev Pharmacol Toxicol. 2003;43:441-461. Epub 2002 Jan 2010.

387. Parham WA, Mehdirad AA, Biermann KM, Fredman CS. Hyperkalemia revisited. Tex Heart Inst J. 2006;33:40-47.

388. Lehnart SE, Ackerman MJ, Benson DW, Jr., Brugada R, Clancy CE, Donahue JK, George AL, Jr., Grant AO, Groft SC, January CT, Lathrop DA, Lederer WJ, Makielski JC, Mohler PJ, Moss A, Nerbonne JM, Olson TM, Przywara DA, Towbin JA, Wang LH, Marks AR. Inherited arrhythmias: A national heart, lung, and blood institute and office of rare diseases workshop consensus report about the diagnosis, phenotyping, molecular mechanisms, and therapeutic approaches for primary cardiomyopathies of gene mutations affecting ion channel function. Circulation. 2007;116:2325-2345.

389. Ruan Y, Liu N, Priori SG. Sodium channel mutations and arrhythmias. Nat Rev Cardiol. 2009;6:337-348. doi: 310.1038/nrcardio.2009.1044.

390. Zimmer T, Surber R. Scn5a channelopathies--an update on mutations and mechanisms. Prog Biophys Mol Biol. 2008;98:120-136. doi: 110.1016/j.pbiomolbio.2008.1010.1005. Epub 2008 Nov 1015.

391. George AL, Jr., Varkony TA, Drabkin HA, Han J, Knops JF, Finley WH, Brown GB, Ward DC, Haas M. Assignment of the human heart tetrodotoxin-resistant voltage-gated na+ channel alpha-subunit gene (scn5a) to band 3p21. Cytogenet Cell Genet. 1995;68:67-70.

392. Wang Q, Shen J, Splawski I, Atkinson D, Li Z, Robinson JL, Moss AJ, Towbin JA, Keating MT. Scn5a mutations associated with an inherited cardiac arrhythmia, long qt syndrome. Cell. 1995;80:805-811.

393. Wang Q, Shen J, Li Z, Timothy K, Vincent GM, Priori SG, Schwartz PJ, Keating MT. Cardiac sodium channel mutations in patients with long qt syndrome, an inherited cardiac arrhythmia. Hum Mol Genet. 1995;4:1603-1607.

394. Chandra R, Starmer CF, Grant AO. Multiple effects of kpq deletion mutation on gating of human cardiac na+ channels expressed in mammalian cells. Am J Physiol. 1998;274:H16431654.

395. Wang DW, Yazawa K, George AL, Jr., Bennett PB. Characterization of human cardiac na+ channel mutations in the congenital long qt syndrome. Proc Natl Acad Sci U $S$ A. 1996;93:13200-13205.

396. Dumaine R, Wang Q, Keating MT, Hartmann HA, Schwartz PJ, Brown AM, Kirsch GE. Multiple mechanisms of na+ channel--linked long-qt syndrome. Circ Res. 1996;78:916-924.

397. Undrovinas AI, Maltsev VA, Kyle JW, Silverman N, Sabbah HN. Gating of the late na+ channel in normal and failing human myocardium. J Mol Cell Cardiol. 2002;34:1477-1489

398. Abriel H, Cabo C, Wehrens XH, Rivolta I, Motoike HK, Memmi M, Napolitano C, Priori SG, Kass RS. Novel arrhythmogenic mechanism revealed by a long-qt syndrome mutation in the cardiac na(+) channel. Circ Res. 2001;88:740-745. 
399. Groenewegen WA, Bezzina CR, van Tintelen JP, Hoorntje TM, Mannens MM, Wilde AA, Jongsma HJ, Rook MB. A novel lqt3 mutation implicates the human cardiac sodium channel domain ivs6 in inactivation kinetics. Cardiovasc Res. 2003;57:1072-1078.

400. Clancy CE, Tateyama M, Liu H, Wehrens XH, Kass RS. Non-equilibrium gating in cardiac na+ channels: An original mechanism of arrhythmia. Circulation. 2003;107:2233-2237. Epub 2003 Apr 2214.

401. Anyukhovsky EP, Sosunov EA, Gainullin RZ, Rosen MR. The controversial m cell. J Cardiovasc Electrophysiol. 1999;10:244-260

402. Liu DW, Gintant GA, Antzelevitch C. Ionic bases for electrophysiological distinctions among epicardial, midmyocardial, and endocardial myocytes from the free wall of the canine left ventricle. Circ Res. 1993;72:671-687.

403. Milberg P, Reinsch N, Wasmer K, Monnig G, Stypmann J, Osada N, Breithardt G, Haverkamp W, Eckardt L. Transmural dispersion of repolarization as a key factor of arrhythmogenicity in a novel intact heart model of lqt3. Cardiovasc Res. 2005;65:397-404.

404. Killeen MJ, Thomas G, Gurung IS, Goddard CA, Fraser JA, Mahaut-Smith MP, Colledge WH, Grace AA, Huang CL. Arrhythmogenic mechanisms in the isolated perfused hypokalaemic murine heart. Acta physiologica (Oxford, England). 2007;189:33-46

405. London B, Baker LC, Petkova-Kirova P, Nerbonne JM, Choi BR, Salama G. Dispersion of repolarization and refractoriness are determinants of arrhythmia phenotype in transgenic mice with long qt. J Physiol. 2007;578:115-129

406. Antzelevitch C, Sicouri S. Clinical relevance of cardiac arrhythmias generated by afterdepolarizations. Role of $\mathrm{m}$ cells in the generation of $\mathrm{u}$ waves, triggered activity and torsade de pointes. J Am Coll Cardiol. 1994;23:259-277.

407. Han J, Moe GK. Non-uniform recovery of excitability in the ventricular muscle. Circ Res. 1964;14:44-60

408. Kuo CS, Reddy CP, Munakata K, Surawicz B. Mechanism of ventricular arrhythmias caused by increased dispersion of repolarization. Eur Heart J. 1985;6 Suppl D:63-70

409. Rosen MR, Wit AL. Arrhythmogenic actions of antiarrhythmic drugs. The American journal of cardiology. 1987;59:10e-18e

410. Antzelevitch C. Heterogeneity and cardiac arrhythmias: An overview. Heart Rhythm. 2007;4:964-972. Epub 2007 Apr 2006.

411. Krishnan SC, Antzelevitch C. Flecainide-induced arrhythmia in canine ventricular epicardium. Phase 2 reentry? Circulation. 1993;87:562-572.

412. Janse MJ. Reentrant arrhythmias In: Fozzard HA, Haber E, Jennings RB, Katz AM, Morgan HE, eds. The heart and cardiovascular system New York: Raven Press; 1991:2055-2094.

413. Brugada P, Wellens HJ. Early afterdepolarizations: Role in conduction block, "prolonged repolarization-dependent reexcitation," and tachyarrhythmias in the human heart. Pacing Clin Electrophysiol. 1985;8:889-896

414. Chen Q, Kirsch GE, Zhang D, Brugada R, Brugada J, Brugada P, Potenza D, Moya A, Borggrefe M, Breithardt G, Ortiz-Lopez R, Wang Z, Antzelevitch C, O'Brien RE, Schulze-Bahr E, Keating MT, Towbin JA, Wang Q. Genetic basis and molecular mechanism for idiopathic ventricular fibrillation. Nature. 1998;392:293-296.

415. Brugada P, Brugada J. Right bundle branch block, persistent st segment elevation and sudden cardiac death: A distinct clinical and electrocardiographic syndrome. A multicenter report. J Am Coll Cardiol. 1992;20:1391-1396.

416. Brugada R, Brugada J, Antzelevitch C, Kirsch GE, Potenza D, Towbin JA, Brugada P. Sodium channel blockers identify risk for sudden death in patients with st-segment elevation and right bundle branch block but structurally normal hearts. Circulation. 2000;101:510-515.

417. Delisle BP, Anson BD, Rajamani S, January CT. Biology of cardiac arrhythmias: Ion channel protein trafficking. Circ Res. 2004;94:1418-1428. 
418. Wang DW, Makita N, Kitabatake A, Balser JR, George AL, Jr. Enhanced na(+) channel intermediate inactivation in brugada syndrome. Circ Res. 2000;87:E37-43.

419. Tan HL, Bink-Boelkens MT, Bezzina CR, Viswanathan PC, Beaufort-Krol GC, van Tintelen PJ, van den Berg MP, Wilde AA, Balser JR. A sodium-channel mutation causes isolated cardiac conduction disease. Nature. 2001;409:1043-1047.

420. Grant AO, Carboni MP, Neplioueva V, Starmer CF, Memmi M, Napolitano C, Priori S. Long qt syndrome, brugada syndrome, and conduction system disease are linked to a single sodium channel mutation. J Clin Invest. 2002;110:1201-1209.

421. Bezzina C, Veldkamp MW, van Den Berg MP, Postma AV, Rook MB, Viersma JW, van Langen IM, Tan-Sindhunata G, Bink-Boelkens MT, van Der Hout AH, Mannens MM, Wilde AA. A single na(+) channel mutation causing both long-qt and brugada syndromes. Circ Res. 1999;85:1206-1213.

422. Benitah JP, Chen Z, Balser JR, Tomaselli GF, Marban E. Molecular dynamics of the sodium channel pore vary with gating: Interactions between p-segment motions and inactivation. $J$ Neurosci. 1999;19:1577-1585.

423. Alings M, Wilde A. "Brugada" syndrome: Clinical data and suggested pathophysiological mechanism. Circulation. 1999;99:666-673.

424. Balser JR. Sodium "channelopathies" and sudden death: Must you be so sensitive? Circ Res. 1999;85:872-874.

425. Rook MB, Evers MM, Vos MA, Bierhuizen MF. Biology of cardiac sodium channel nav1.5 expression. Cardiovasc Res. 2012;93:12-23

426. Kistner U, Wenzel BM, Veh RW, Cases-Langhoff C, Garner AM, Appeltauer U, Voss B, Gundelfinger ED, Garner CC. Sap90, a rat presynaptic protein related to the product of the drosophila tumor suppressor gene dlg-a. J Biol Chem. 1993;268:4580-4583.

427. Godreau D, Vranckx R, Maguy A, Goyenvalle C, Hatem SN. Different isoforms of synapseassociated protein, sap97, are expressed in the heart and have distinct effects on the voltagegated k+ channel kv1.5. J Biol Chem. 2003;278:47046-47052. Epub 42003 Sep 47010.

428. Godreau D, Vranckx R, Maguy A, Rucker-Martin C, Goyenvalle C, Abdelshafy S, Tessier S, Couetil JP, Hatem SN. Expression, regulation and role of the maguk protein sap-97 in human atrial myocardium. Cardiovasc Res. 2002;56:433-442.

429. Bezprozvanny I, Maximov A. Classification of pdz domains. FEBS Lett. 2001;509:457-462.

430. Kim E, Sheng M. Pdz domain proteins of synapses. Nat Rev Neurosci. 2004;5:771-781.

431. Harris BZ, Lim WA. Mechanism and role of pdz domains in signaling complex assembly.J Cell Sci. 2001;114:3219-3231.

432. Abi-Char J, Maguy A, Coulombe A, Balse E, Ratajczak P, Samuel JL, Nattel S, Hatem SN. Membrane cholesterol modulates kv1.5 potassium channel distribution and function in rat cardiomyocytes. J Physiol. 2007;582:1205-1217. Epub 2007 May 1224.

433. Newey SE, Benson MA, Ponting CP, Davies KE, Blake DJ. Alternative splicing of dystrobrevin regulates the stoichiometry of syntrophin binding to the dystrophin protein complex. Curr Biol. 2000;10:1295-1298.

434. Hashemi SM, Hund TJ, Mohler PJ. Cardiac ankyrins in health and disease. J Mol Cell Cardiol. 2009;47:203-209. doi: 210.1016/j.yjmcc.2009.1004.1010. Epub 2009 Apr 1024.

435. Curran J, Mohler PJ. Coordinating electrical activity of the heart: Ankyrin polypeptides in human cardiac disease. Expert Opin Ther Targets. 2011;15:789-801

436. Mohler PJ, Hoffman JA, Davis JQ, Abdi KM, Kim CR, Jones SK, Davis LH, Roberts KF, Bennett V. Isoform specificity among ankyrins. An amphipathic alpha-helix in the divergent regulatory domain of ankyrin-b interacts with the molecular co-chaperone hdj1/hsp40. J Biol Chem. 2004;279:25798-25804. Epub 22004 Apr 25799. 
437. Mohler PJ, Splawski I, Napolitano C, Bottelli G, Sharpe L, Timothy K, Priori SG, Keating MT, Bennett V. A cardiac arrhythmia syndrome caused by loss of ankyrin-b function. Proc Natl Acad Sci U S A. 2004;101:9137-9142. Epub 2004 Jun 9133.

438. Mohler PJ, Schott JJ, Gramolini AO, Dilly KW, Guatimosim S, duBell WH, Song LS, Haurogne K, Kyndt F, Ali ME, Rogers TB, Lederer WJ, Escande D, Le Marec H, Bennett V. Ankyrin-b mutation causes type 4 long-qt cardiac arrhythmia and sudden cardiac death. Nature. 2003;421:634-639.

439. Mohler PJ, Wehrens XH. Mechanisms of human arrhythmia syndromes: Abnormal cardiac macromolecular interactions. Physiology (Bethesda). 2007;22:342-350.

440. Mohler PJ, Rivolta I, Napolitano C, LeMaillet G, Lambert S, Priori SG, Bennett V. Nav1.5 e1053k mutation causing brugada syndrome blocks binding to ankyrin-g and expression of nav1.5 on the surface of cardiomyocytes. Proc Natl Acad Sci U S A. 2004;101:17533-17538. Epub 12004 Dec 17533.

441. Vatta M, Towbin JA. Mutations in kcne1 in long qt syndrome (lqts): Insights into mechanism of lqts and drug sensitivity? Heart Rhythm. 2006;3:1041-1042. Epub 2006 Jul 1021.

442. Sotgia F, Lee JK, Das K, Bedford M, Petrucci TC, Macioce P, Sargiacomo M, Bricarelli FD, Minetti C, Sudol M, Lisanti MP. Caveolin-3 directly interacts with the c-terminal tail of beta dystroglycan. Identification of a central ww-like domain within caveolin family members. $J$ Biol Chem. 2000;275:38048-38058.

443. Doyle DD, Goings G, Upshaw-Earley J, Ambler SK, Mondul A, Palfrey HC, Page E. Dystrophin associates with caveolae of rat cardiac myocytes: Relationship to dystroglycan. Circ Res. 2000;87:480-488.

444. Cronk LB, Ye B, Kaku T, Tester DJ, Vatta M, Makielski JC, Ackerman MJ. Novel mechanism for sudden infant death syndrome: Persistent late sodium current secondary to mutations in caveolin-3. Heart Rhythm. 2007;4:161-166. Epub 2006 Dec 2006.

445. Cohen AW, Hnasko R, Schubert W, Lisanti MP. Role of caveolae and caveolins in health and disease. Physiol Rev. 2004;84:1341-1379.

446. Abriel H. Cardiac sodium channel na(v)1.5 and interacting proteins: Physiology and pathophysiology. J Mol Cell Cardiol. 2010;48:2-11

447. Gialama F, Prezerakos P, Maniadakis N. The cost effectiveness of implantable cardioverter defibrillators: A systematic review of economic evaluations. Applied health economics and health policy. 2013

448. Borne RT, Varosy PD, Masoudi FA. Implantable cardioverter-defibrillator shocks: Epidemiology, outcomes, and therapeutic approaches. JAMA internal medicine. 2013;173:859-865

449. Bunch TJ, Anderson JL. Adjuvant antiarrhythmic therapy in patients with implantable cardioverter defibrillators. American journal of cardiovascular drugs : drugs, devices, and other interventions. 2013

450. Grant AO, Jr. On the mechanism of action of antiarrhythmic agents. Am Heart J. 1992;123:1130-1136.

451. Grant AO, Starmer CF, Strauss HC. Antiarrhythmic drug action. Blockade of the inward sodium current. Circ Res. 1984;55:427-439.

452. Whalley DW, Wendt DJ, Grant AO. Basic concepts in cellular cardiac electrophysiology: Part ii: Block of ion channels by antiarrhythmic drugs. Pacing Clin Electrophysiol. 1995;18:16861704

453. Sheldon RS, Hill RJ, Duff HJ. Antiarrhythmic drugs and the cardiac sodium channel: Current models. Clinical chemistry. 1989;35:748-754

454. Carmeliet E, Mubagwa K. Antiarrhythmic drugs and cardiac ion channels: Mechanisms of action. Prog Biophys Mol Biol. 1998;70:1-72. 
455. Hondeghem LM, Snyders DJ. Class iii antiarrhythmic agents have a lot of potential but a long way to go. Reduced effectiveness and dangers of reverse use dependence. Circulation. 1990;81:686-690.

456. Machida T, Hashimoto N, Kuwahara I, Ogino Y, Matsuura J, Yamamoto W, Itano Y, Zamma A, Matsumoto R, Kamon J, Kobayashi T, Ishiwata N, Yamashita T, Ogura T, Nakaya H. Effects of a highly selective acetylcholine-activated $\mathrm{k}+$ channel blocker on experimental atrial fibrillation. Circ Arrhythm Electrophysiol. 2011;4:94-102

457. Dobrev D, Friedrich A, Voigt N, Jost N, Wettwer E, Christ T, Knaut M, Ravens U. The g proteingated potassium current $\mathrm{i}(\mathrm{k}, \mathrm{ach})$ is constitutively active in patients with chronic atrial fibrillation. Circulation. 2005;112:3697-3706

458. Hondeghem LM, Katzung BG. Antiarrhythmic agents: The modulated receptor mechanism of action of sodium and calcium channel-blocking drugs. Annu Rev Pharmacol Toxicol. 1984;24:387-423.

459. Starmer CF, Grant AO, Strauss HC. Mechanisms of use-dependent block of sodium channels in excitable membranes by local anesthetics. Biophys J. 1984;46:15-27

460. Howard PA. Azithromycin-induced proarrhythmia and cardiovascular death. The Annals of pharmacotherapy. 2013;47:1547-1551

461. Volberg WA, Koci BJ, Su W, Lin J, Zhou J. Blockade of human cardiac potassium channel human ether-a-go-go-related gene (herg) by macrolide antibiotics. The Journal of pharmacology and experimental therapeutics. 2002;302:320-327

462. Gysel M, Vieweg WVR, Hasnain M, Hancox JC, Kunanithy V, Baranchuk A. Torsades de pointes following clarithromycin treatment. Expert Review of Cardiovascular Therapy. 2013;11:485-493

463. Funk KA, Bostwick JR. A comparison of the risk of qt prolongation among ssris. The Annals of pharmacotherapy. 2013;47:1330-1341

464. Rampe D, Murawsky MK, Grau J, Lewis EW. The antipsychotic agent sertindole is a high affinity antagonist of the human cardiac potassium channel herg. The Journal of pharmacology and experimental therapeutics. 1998;286:788-793

465. Nattel S. The molecular and ionic specificity of antiarrhythmic drug actions. Journal of Cardiovascular Electrophysiology. 1999;10:272-282

466. Anderson JL, Stewart JR, Perry BA, Van Hamersveld DD, Johnson TA, Conard GJ, Chang SF, Kvam DC, Pitt B. Oral flecainide acetate for the treatment of ventricular arrhythmias. The New England journal of medicine. 1981;305:473-477

467. Flecainide versus quinidine for treatment of chronic ventricular arrhythmias. A multicenter clinical trial. Circulation. 1983;67:1117-1123

468. Treatment of resistant ventricular tachycardia with flecainide acetate. Flecainide ventricular tachycardia study group. The American journal of cardiology. 1986;57:1299-1304

469. Neuss H, Schlepper M. Long-term efficacy and safety of flecainide for supraventricular tachycardia. The American journal of cardiology. 1988;62:56d-61d

470. Nathan AW, Hellestrand KJ, Bexton RS, Banim SO, Spurrell RA, Camm AJ. Proarrhythmic effects of the new antiarrhythmic agent flecainide acetate. American heart journal. 1984;107:222-228

471. Legrand V, Vandormael M, Collignon P, Kulbertus HE. Hemodynamic effects of a new antiarrhythmic agent, flecainide ( $\mathrm{r}-818)$, in coronary heart disease. The American journal of cardiology. 1983;51:422-426

472. Anderson JL, Lutz JR, Allison SB. Electrophysiologic and antiarrhythmic effects of oral flecainide in patients with inducible ventricular tachycardia. J Am Coll Cardiol. 1983;2:105114

473. Flecainide and cast. Lancet. 1989;2:481-482 
474. Preliminary report: Effect of encainide and flecainide on mortality in a randomized trial of arrhythmia suppression after myocardial infarction. New England Journal of Medicine. 1989;321:406-412

475. Starmer CF. How antiarrhythmic drugs increase the rate of sudden cardiac death. International Journal of Bifurcation and Chaos. 2002;12:1953-1968

476. Sanderson J. The sword of damocles. Lancet. 1996;348:2-3

477. Allessie MA, Bonke FI, Schopman FJ. Circus movement in rabbit atrial muscle as a mechanism of tachycardia. Ii. The role of nonuniform recovery of excitability in the occurrence of unidirectional block, as studied with multiple microelectrodes. Circ Res. 1976;39:168-177

478. Arnsdorf MF. Basic understanding of the electrophysiologic actions of antiarrhythmic drugs. Sources, sinks, and matrices of information. Med Clin North Am. 1984;68:1247-1280.

479. Campbell TJ, Vaughan Williams EM. Voltage- and time-dependent depression of maximum rate of depolarisation of guinea-pig ventricular action potentials by two new antiarrhythmic drugs, flecainide and lorcainide. Cardiovasc Res. 1983;17:251-258.

480. Selzer A, Wray HW. Quinidine syncope. Paroxysmal ventricular fibrillation occurring during treatment of chronic atrial arrhythmias. Circulation. 1964;30:17-26

481. Horowitz LN, Zipes DP, Bigger JT, Jr., Campbell RW, Morganroth J, Podrid PJ, Rosen MR, Woosley RL. Proarrhythmia, arrhythmogenesis or aggravation of arrhythmia--a status report, 1987. The American journal of cardiology. 1987;59:54e-56e

482. Nattel S. Experimental evidence for proarrhythmic mechanisms of antiarrhythmic drugs. Cardiovasc Res. 1998;37:567-577

483. Ranger S, Talajic M, Lemery R, Roy D, Nattel S. Amplification of flecainide-induced ventricular conduction slowing by exercise. A potentially significant clinical consequence of use-dependent sodium channel blockade. Circulation. 1989;79:1000-1006.

484. Ranger S, Nattel S. Determinants and mechanisms of flecainide-induced promotion of ventricular tachycardia in anesthetized dogs. Circulation. 1995;92:1300-1311

485. Restivo M, Yin H, Caref EB, Patel AI, Ndrepepa G, Avitable MJ, Assadi MA, Isber N, el-Sherif N. Reentrant arrhythmias in the subacute infarction period. The proarrhythmic effect of flecainide acetate on functional reentrant circuits. Circulation. 1995;91:1236-1246.

486. Coromilas J, Saltman AE, Waldecker B, Dillon SM, Wit AL. Electrophysiological effects of flecainide on anisotropic conduction and reentry in infarcted canine hearts. Circulation. 1995;91:2245-2263

487. Arnsdorf MF, Sawicki GJ. Flecainide and the electrophysiologic matrix: The effects of flecainide acetate on the determinants of cardiac excitability in sheep purkinje fibers. $J$ Cardiovasc Electrophysiol. 1996;7:1172-1182.

488. Elharrar V, Zipes DP. Cardiac electrophysiologic alterations during myocardial ischemia. The American journal of physiology. 1977;233:H329-345

489. Nattel S, Pedersen DH, Zipes DP. Alterations in regional myocardial distribution and arrhythmogenic effects of aprindine produced by coronary artery occlusion in the dog. Cardiovasc Res. 1981;15:80-85

490. Elharrar V, Gaum WE, Zipes DP. Effect of drugs on conduction delay and incidence of ventricular arrhythmias induced by acute coronary occlusion in dogs. The American journal of cardiology. 1977;39:544-549

491. Carson DL, Cardinal R, Savard P, Vasseur C, Nattel S, Lambert C, Nadeau R. Relationship between an arrhythmogenic action of lidocaine and its effects on excitation patterns in acutely ischemic porcine myocardium. J Cardiovasc Pharmacol. 1986;8:126-136

492. Starmer CF, Lastra AA, Nesterenko VV, Grant AO. Proarrhythmic response to sodium channel blockade. Theoretical model and numerical experiments. Circulation. 1991;84:1364-1377. 
493. Nattel S, Waters D. What is an antiarrhythmic drug? From clinical trials to fundamental concepts. The American journal of cardiology. 1990;66:96-99

494. Greenberg HM, Dwyer EM, Jr., Hochman JS, Steinberg JS, Echt DS, Peters RW. Interaction of ischaemia and encainide/flecainide treatment: A proposed mechanism for the increased mortality in cast i. British heart journal. 1995;74:631-635

495. Nesterenko VV, Lastra AA, Rosenshtraukh LV, Starmer CF. A proarrhythmic response to sodium channel blockade: Modulation of the vulnerable period in guinea pig ventricular myocardium. J Cardiovasc Pharmacol. 1992;19:810-820

496. Starmer CF, Lancaster AR, Lastra AA, Grant AO. Cardiac instability amplified by usedependent na channel blockade. The American journal of physiology. 1992;262:H1305-1310

497. Brugada J, Boersma L, Kirchhof C, Allessie M. Proarrhythmic effects of flecainide. Experimental evidence for increased susceptibility to reentrant arrhythmias. Circulation. 1991;84:1808-1818

498. Antzelevitch C. Drug-induced spatial dispersion of repolarization. Cardiology journal. 2008;15:100-121

499. Krishnan SC, Antzelevitch C. Sodium channel block produces opposite electrophysiological effects in canine ventricular epicardium and endocardium. Circ Res. 1991;69:277-291.

500. Nawrotzki R, Blake DJ, Davies KE. The genetic basis of neuromuscular disorders. Trends Genet. 1996;12:294-298.

501. James JF, Hewett TE, Robbins J. Cardiac physiology in transgenic mice. Circ Res. 1998;82:407-415.

502. Weiss JN, Qu Z, Chen PS, Lin SF, Karagueuzian HS, Hayashi H, Garfinkel A, Karma A. The dynamics of cardiac fibrillation. Circulation. 2005;112:1232-1240.

503. Antzelevitch C, Sicouri S, Litovsky SH, Lukas A, Krishnan SC, Di Diego JM, Gintant GA, Liu DW. Heterogeneity within the ventricular wall. Electrophysiology and pharmacology of epicardial, endocardial, and m cells. Circ Res. 1991;69:1427-1449.

504. Kass DA, Hare JM, Georgakopoulos D. Murine cardiac function: A cautionary tail. Circ Res. 1998;82:519-522.

505. Efimov IR, Nikolski VP, Salama G. Optical imaging of the heart. Circ Res. 2004;95:21-33

506. Guerrero PA, Schuessler RB, Davis LM, Beyer EC, Johnson CM, Yamada KA, Saffitz JE. Slow ventricular conduction in mice heterozygous for a connexin43 null mutation. J Clin Invest. 1997;99:1991-1998.

507. Thomas SA, Schuessler RB, Berul CI, Beardslee MA, Beyer EC, Mendelsohn ME, Saffitz JE. Disparate effects of deficient expression of connexin43 on atrial and ventricular conduction: Evidence for chamber-specific molecular determinants of conduction. Circulation. 1998;97:686-691.

508. Bernstein SA, Duggirala S, Floberg M, Elfvendal P, Kuznekoff LM, Lader JM, Vasquez C, Morley GE. Spatiotemporal electrophysiological changes in a murine ablation model. Europace. 2011;13:1494-1500

509. Ding C, Gepstein L, Nguyen DT, Wilson E, Hulley G, Beaser A, Lee RJ, Olgin J. High-resolution optical mapping of ventricular tachycardia in rats with chronic myocardial infarction. Pacing Clin Electrophysiol. 2010;33:687-695

510. Mills WR, Mal N, Forudi F, Popovic ZB, Penn MS, Laurita KR. Optical mapping of late myocardial infarction in rats. Am J Physiol Heart Circ Physiol. 2006;290:H1298-1306. Epub 2005 Oct 1297.

511. Campbell TJ. Kinetics of onset of rate-dependent effects of class i antiarrhythmic drugs are important in determining their effects on refractoriness in guinea-pig ventricle, and provide a theoretical basis for their subclassification. Cardiovasc Res. 1983;17:344-352.

512. Janse MJ. Putting the sicilian gambit to the test. Eur Heart J. 1992;13:30-37. 
513. Weirich J, Hohnloser S, Antoni H. Differential analysis of frequency-dependent effects of antiarrhythmic drugs: Importance of the saturation behavior of frequency-dependent sodium-channel blockade. J Cardiovasc Pharmacol. 1992;20:S8-16.

514. Herron TJ, Lee P, Jalife J. Optical imaging of voltage and calcium in cardiac cells \& tissues. Circ Res. 2012;110:609-623

515. Lang D, Sulkin M, Lou Q, Efimov IR. Optical mapping of action potentials and calcium transients in the mouse heart. Journal of visualized experiments : JoVE. 2011

516. Girouard SD, Laurita KR, Rosenbaum DS. Unique properties of cardiac action potentials recorded with voltage-sensitive dyes. J Cardiovasc Electrophysiol. 1996;7:1024-1038

517. Salzberg BM, Obaid AL, Bezanilla F. Microsecond response of a voltage-sensitive merocyanine dye: Fast voltage-clamp measurements on squid giant axon. The Japanese journal of physiology. 1993;43 Suppl 1:S37-41

518. Kanaporis G, Martisiene I, Jurevicius J, Vosyliute R, Navalinskas A, Treinys R, Matiukas A, Pertsov AM. Optical mapping at increased illumination intensities. Journal of biomedical optics. 2012;17:96007-96001

519. Preuss S, Stein W. Comparison of two voltage-sensitive dyes and their suitability for longterm imaging of neuronal activity. PloS one. 2013;8:e75678

520. Sano T, Takayama N, Shimamoto T. Directional difference of conduction velocity in the cardiac ventricular syncytium studied by microelectrodes. Circ Res. 1959;7:262-267.

521. Knollmann BC, Katchman AN, Franz MR. Monophasic action potential recordings from intact mouse heart: Validation, regional heterogeneity, and relation to refractoriness. J Cardiovasc Electrophysiol. 2001;12:1286-1294

522. Swift LM, Asfour H, Posnack NG, Arutunyan A, Kay MW, Sarvazyan N. Properties of blebbistatin for cardiac optical mapping and other imaging applications. Pflugers Arch. 2012;464:503-512

523. Fedorov VV, Lozinsky IT, Sosunov EA, Anyukhovsky EP, Rosen MR, Balke CW, Efimov IR. Application of blebbistatin as an excitation-contraction uncoupler for electrophysiologic study of rat and rabbit hearts. Heart Rhythm. 2007;4:619-626

524. Ebner TJ, Chen G. Use of voltage-sensitive dyes and optical recordings in the central nervous system. Progress in neurobiology. 1995;46:463-506

525. Loew LM, Cohen LB, Dix J, Fluhler EN, Montana V, Salama G, Wu JY. A naphthyl analog of the aminostyryl pyridinium class of potentiometric membrane dyes shows consistent sensitivity in a variety of tissue, cell, and model membrane preparations. The Journal of membrane biology. 1992;130:1-10

526. Loew LM. Design and use of organic voltage sensitive dyes. In: Canepari M, Zecevic D, eds. Membrane potential imaging in the nervous system: Methods and applications. Springer Science; 2010:13-23.

527. Loew LM, Simpson LL. Charge-shift probes of membrane potential: A probable electrochromic mechanism for p-aminostyrylpyridinium probes on a hemispherical lipid bilayer. Biophys J. 1981;34:353-365

528. Chen TW, Lin BJ, Brunner E, Schild D. In situ background estimation in quantitative fluorescence imaging. Biophys J. 2006;90:2534-2547. Epub 2005 Dec 2530.

529. Sung D, Mills RW, Schettler J, Narayan SM, Omens JH, McCulloch AD. Ventricular filling slows epicardial conduction and increases action potential duration in an optical mapping study of the isolated rabbit heart. J Cardiovasc Electrophysiol. 2003;14:739-749.

530. Benson AP, Bernus O, Dierckx H, Gilbert SH, Greenwood JP, Holden AV, Mohee K, Plein S, Radjenovic A, Ries ME, Smith GL, Sourbron S, Walton RD. Construction and validation of anisotropic and orthotropic ventricular geometries for quantitative predictive cardiac electrophysiology. Interface Focus. 2011;1:101-116 
531. Fitzgibbon AW, Pilu M, Fisher RB. Direct least square fitting of ellipses. IEEE Transactions on Pattern Analysis and Machine Intelligence. 1999;21(5):476-480

532. Mourad A, Nash MP. Method for quantifiying conduction velocity during ventricular fibrillation. Phys Rev E Stat Nonlin Soft Matter Phys. 2007;75:011914. Epub 012007 Jan 011917.

533. Salama G, Kanai A, Efimov IR. Subthreshold stimulation of purkinje fibers interrupts ventricular tachycardia in intact hearts. Experimental study with voltage-sensitive dyes and imaging techniques. Circ Res. 1994;74:604-619.

534. Fenton F, Karma A. Vortex dynamics in three-dimensional continuous myocardium with fiber rotation: Filament instability and fibrillation. Chaos. 1998;8:20-47.

535. Kaab S, Nuss HB, Chiamvimonvat N, O'Rourke B, Pak PH, Kass DA, Marban E, Tomaselli GF. Ionic mechanism of action potential prolongation in ventricular myocytes from dogs with pacing-induced heart failure. Circ Res. 1996;78:262-273.

536. Pu J, Boyden PA. Alterations of na+ currents in myocytes from epicardial border zone of the infarcted heart. A possible ionic mechanism for reduced excitability and postrepolarization refractoriness. Circ Res. 1997;81:110-119.

537. Spach MS, Heidlage JF. The stochastic nature of cardiac propagation at a microscopic level. Electrical description of myocardial architecture and its application to conduction. Circ Res. 1995;76:366-380.

538. Antzelevitch C. Role of spatial dispersion of repolarization in inherited and acquired sudden cardiac death syndromes. Am J Physiol Heart Circ Physiol. 2007;293:H2024-2038. Epub 2007 Jun 2022.

539. Fast VG, Darrow BJ, Saffitz JE, Kleber AG. Anisotropic activation spread in heart cell monolayers assessed by high-resolution optical mapping. Role of tissue discontinuities. Circ Res. 1996;79:115-127.

540. The sicilian gambit. A new approach to the classification of antiarrhythmic drugs based on their actions on arrhythmogenic mechanisms. Task force of the working group on arrhythmias of the european society of cardiology. Circulation. 1991;84:1831-1851.

541. Campbell TJ, Williams KM. Therapeutic drug monitoring: Antiarrhythmic drugs. Br J Clin Pharmacol. 1998;46:307-319.

542. Franz MR. Bridging the gap between basic and clinical electrophysiology: What can be learned from monophasic action potential recordings? J Cardiovasc Electrophysiol. 1994;5:699-710.

543. Jin H, Lyon AR, Akar FG. Arrhythmia mechanisms in the failing heart. Pacing Clin Electrophysiol. 2008;31:1048-1056. doi: 1010.1111/j.1540-8159.2008.01134.x.

544. Scholz H. Classification and mechanism of action of antiarrhythmic drugs. Fundam Clin Pharmacol. 1994;8:385-390.

545. Chay TR. Why are some antiarrhythmic drugs proarrhythmic? Cardiac arrhythmia study by bifurcation analysis. J Electrocardiol. 1995;28:191-197.

546. Ulbricht $\mathrm{W}$, Wagner $\mathrm{HH}$. The influence of ph on equilibrium effects of tetrodotoxin on myelinated nerve fibres of rana esculenta. J Physiol. 1975;252:159-184.

547. Pappone PA. Voltage-clamp experiments in normal and denervated mammalian skeletal muscle fibres.J Physiol. 1980;306:377-410.

548. Arbel E, Sasyniuk B, Moe GK. Supernormal ventricular conduction in the dog heart (abstr.). Fed Proc. 1971;30:553

549. Spear JF, Moore EN. Supernormal excitability and conduction in the his-purkinje system of the dog. Circ Res. 1974;35:782-792.

550. Laughner JI, Ng FS, Sulkin MS, Arthur RM, Efimov IR. Processing and analysis of cardiac optical mapping data obtained with potentiometric dyes. Am J Physiol Heart Circ Physiol. 2012;303:H753-765 
551. Kleber AG, Janse MJ, Fast VG. Normal and abnormal conduction in the heart. Handbook of physiology. The cardiovascular system : The heart. Bestheda: American Physiological Society; 2002:455-530.

552. Spach MS, Kootsey JM, Sloan JD. Active modulation of electrical coupling between cardiac cells of the dog. A mechanism for transient and steady state variations in conduction velocity. Circ Res. 1982;51:347-362.

553. Iida M, Kodama I, Toyama J. Negative dromotropic effects of class i antiarrhythmic drugs in anisotropic ventricular muscle. Cardiovasc Res. 1996;31:640-650

554. Antzelevitch C, Shimizu W, Yan GX, Sicouri S. Cellular basis for qt dispersion. J Electrocardiol. 1998;30:168-175.

555. Myles RC, Bernus O, Burton FL, Cobbe SM, Smith GL. Effect of activation sequence on transmural patterns of repolarization and action potential duration in rabbit ventricular myocardium. Am J Physiol Heart Circ Physiol. 2010;299:H1812-1822

556. Sampson KJ, Henriquez CS. Interplay of ionic and structural heterogeneity on functional action potential duration gradients: Implications for arrhythmogenesis. Chaos (Woodbury, N.Y.). 2002;12:819-828

557. Lesh MD, Pring M, Spear JF. Cellular uncoupling can unmask dispersion of action potential duration in ventricular myocardium. A computer modeling study. Circ Res. 1989;65:14261440

558. Watanabe H, Steele DS, Knollmann BC. Mechanism of antiarrhythmic effects of flecainide in catecholaminergic polymorphic ventricular tachycardia. Circ Res. 2011;109:712-713

559. Zipes DP. Proarrhythmic effects of antiarrhythmic drugs. Am J Cardiol. 1987;59:2E-9E

560. Burton FL, Cobbe SM. Dispersion of ventricular repolarization and refractory period. Cardiovasc Res. 2001;50:10-23.

561. Kodama I, Honjo H, Kamiya K, Toyama J. Two types of sodium channel block by class-i antiarrhythmic drugs studied by using vmax of action potential in single ventricular myocytes. J Mol Cell Cardiol. 1990;22:1-12

562. Ranger S, Sheldon R, Fermini B, Nattel S. Modulation of flecainide's cardiac sodium channel blocking actions by extracellular sodium: A possible cellular mechanism for the action of sodium salts in flecainide cardiotoxicity. The Journal of pharmacology and experimental therapeutics. 1993;264:1160-1167

563. Catterall WA. Structure and function of voltage-gated ion channels. Annu Rev Biochem. 1995;64:493-531.

564. Kambouris NG, Nuss HB, Johns DC, Tomaselli GF, Marban E, Balser JR. Phenotypic characterization of a novel long-qt syndrome mutation (r1623q) in the cardiac sodium channel. Circulation. 1998;97:640-644

565. Nagatomo T, January CT, Makielski JC. Preferential block of late sodium current in the lqt3 deltakpq mutant by the class i(c) antiarrhythmic flecainide. Mol Pharmacol. 2000;57:101107.

566. Liu H, Tateyama M, Clancy CE, Abriel H, Kass RS. Channel openings are necessary but not sufficient for use-dependent block of cardiac na(+) channels by flecainide: Evidence from the analysis of disease-linked mutations. J Gen Physiol. 2002;120:39-51.

567. Windle JR, Geletka RC, Moss AJ, Zareba W, Atkins DL. Normalization of ventricular repolarization with flecainide in long qt syndrome patients with scn5a:Deltakpq mutation. Annals of noninvasive electrocardiology : the official journal of the International Society for Holter and Noninvasive Electrocardiology, Inc. 2001;6:153-158

568. Moss AJ, Windle JR, Hall WJ, Zareba W, Robinson JL, McNitt S, Severski P, Rosero S, Daubert JP, Qi M, Cieciorka M, Manalan AS. Safety and efficacy of flecainide in subjects with long qt-3 syndrome (deltakpq mutation): A randomized, double-blind, placebo-controlled clinical 
trial. Annals of noninvasive electrocardiology : the official journal of the International Society for Holter and Noninvasive Electrocardiology, Inc. 2005;10:59-66

569. Wang DW, Yazawa K, Makita N, George AL, Jr., Bennett PB. Pharmacological targeting of long qt mutant sodium channels. J Clin Invest. 1997;99:1714-1720.

570. Priori SG, Napolitano C, Schwartz PJ, Bloise R, Crotti L, Ronchetti E. The elusive link between lqt3 and brugada syndrome: The role of flecainide challenge. Circulation. 2000;102:945-947.

571. Anno T, Hondeghem LM. Interactions of flecainide with guinea pig cardiac sodium channels. Importance of activation unblocking to the voltage dependence of recovery. Circ Res. 1990;66:789-803.

572. Knisley SB, Hill BC. Effects of bipolar point and line stimulation in anisotropic rabbit epicardium: Assessment of the critical radius of curvature for longitudinal block. IEEE transactions on bio-medical engineering. 1995;42:957-966

573. Cabo C, Pertsov AM, Baxter WT, Davidenko JM, Gray RA, Jalife J. Wave-front curvature as a cause of slow conduction and block in isolated cardiac muscle. Circ Res. 1994;75:1014-1028.

574. Schwartz PJ, Priori SG, Locati EH, Napolitano C, Cantu F, Towbin JA, Keating MT, Hammoude $\mathrm{H}$, Brown AM, Chen LS. Long qt syndrome patients with mutations of the scn5a and herg genes have differential responses to na+ channel blockade and to increases in heart rate. Implications for gene-specific therapy. Circulation. 1995;92:3381-3386.

575. Yan GX, Antzelevitch C. Cellular basis for the normal t wave and the electrocardiographic manifestations of the long-qt syndrome. Circulation. 1998;98:1928-1936

576. Benhorin J, Taub R, Goldmit M, Kerem B, Kass RS, Windman I, Medina A. Effects of flecainide in patients with new scn5a mutation: Mutation-specific therapy for long-qt syndrome? Circulation. 2000;101:1698-1706.

577. Beinart R, Michailidis A, Gurevitz OT, Glikson M. Is flecainide dangerous in long qt-3 patients? Pacing Clin Electrophysiol. 2009;32:143-145. doi: 110.1111/j.15408159.2009.02190.x.

578. Schotten U, Verheule S, Kirchhof P, Goette A. Pathophysiological mechanisms of atrial fibrillation: A translational appraisal. Physiol Rev. 2011;91:265-325

579. Downar E, Janse MJ, Durrer D. The effect of acute coronary artery occlusion on subepicardial transmembrane potentials in the intact porcine heart. Circulation. 1977;56:217-224

580. Kleber AG, Janse MJ, Wilms-Schopmann FJ, Wilde AA, Coronel R. Changes in conduction velocity during acute ischemia in ventricular myocardium of the isolated porcine heart. Circulation. 1986;73:189-198

581. Wilde AA, Kleber AG. The combined effects of hypoxia, high $\mathrm{k}+$, and acidosis on the intracellular sodium activity and resting potential in guinea pig papillary muscle. Circ Res. 1986;58:249-256

582. Zeng J, Laurita KR, Rosenbaum DS, Rudy Y. Two components of the delayed rectifier k+ current in ventricular myocytes of the guinea pig type. Theoretical formulation and their role in repolarization. Circ Res. 1995;77:140-152

583. Colussi C, Berni R, Rosati J, Straino S, Vitale S, Spallotta F, Baruffi S, Bocchi L, Delucchi F, Rossi S, Savi M, Rotili D, Quaini F, Macchi E, Stilli D, Musso E, Mai A, Gaetano C, Capogrossi MC. The histone deacetylase inhibitor suberoylanilide hydroxamic acid reduces cardiac arrhythmias in dystrophic mice. Cardiovasc Res. 2010;87:73-82

584. Sanford JL, Edwards JD, Mays TA, Gong B, Merriam AP, Rafael-Fortney JA. Claudin-5 localizes to the lateral membranes of cardiomyocytes and is altered in utrophin/dystrophin-deficient cardiomyopathic mice. J Mol Cell Cardiol. 2005;38:323-332

585. Shaw RM, Rudy Y. Ionic mechanisms of propagation in cardiac tissue. Roles of the sodium and l-type calcium currents during reduced excitability and decreased gap junction coupling. Circ Res. 1997;81:727-741 
586. Draper MH, Mya-Tu M. A comparison of the conduction velocity in cardiac tissues of various mammals. Quarterly journal of experimental physiology and cognate medical sciences. 1959;44:91-109

587. Rosen MR, Legato MJ, Weiss RM. Developmental changes in impulse conduction in the canine heart. The American journal of physiology. 1981;240:H546-554

588. Boersma L, Brugada J, Schalij MJ, Kirchhof C, Allessie M. The effects of $\mathrm{k}+$ on anisotropic conduction in sheets of perfused rabbit ventricular epicardium. Journal of Cardiovascular Electrophysiology. 1991;2:492-502

589. Spach M, Heidlage JF, Dolber PC, Barr RC. Changes in anisotropic conduction caused by remodeling cell size and the cellular distribution of gap junctions and na channels. Journal of electrocardiology. 2001;34:69-76

590. Fast VG, Kleber AG. Anisotropic conduction in monolayers of neonatal rat heart cells cultured on collagen substrate. Circ Res. 1994;75:591-595

591. Bukauskas FF, Elfgang C, Willecke K, Weingart R. Biophysical properties of gap junction channels formed by mouse connexin40 in induced pairs of transfected human hela cells. Biophys J. 1995;68:2289-2298

592. Valiunas V, Bukauskas FF, Weingart R. Conductances and selective permeability of connexin43 gap junction channels examined in neonatal rat heart cells. Circ Res. 1997;80:708-719

593. Henriquez AP, Vogel R, Muller-Borer BJ, Henriquez CS, Weingart R, Cascio WE. Influence of dynamic gap junction resistance on impulse propagation in ventricular myocardium: A computer simulation study. Biophys J. 2001;81:2112-2121

594. Rudisuli A, Weingart R. Electrical properties of gap junction channels in guinea-pig ventricular cell pairs revealed by exposure to heptanol. Pflugers Arch. 1989;415:12-21

595. Hoyt RH, Cohen ML, Saffitz JE. Distribution and three-dimensional structure of intercellular junctions in canine myocardium. Circ Res. 1989;64:563-574

596. Cooklin M, Wallis WR, Sheridan DJ, Fry CH. Changes in cell-to-cell electrical coupling associated with left ventricular hypertrophy. Circ Res. 1997;80:765-771

597. Poelzing S, Rosenbaum DS. Altered connexin43 expression produces arrhythmia substrate in heart failure. Am J Physiol Heart Circ Physiol. 2004;287:H1762-1770

598. Akar FG, Nass RD, Hahn S, Cingolani E, Shah M, Hesketh GG, DiSilvestre D, Tunin RS, Kass DA, Tomaselli GF. Dynamic changes in conduction velocity and gap junction properties during development of pacing-induced heart failure. Am J Physiol Heart Circ Physiol. 2007;293:H1223-1230. Epub 2007 Apr 1213.

599. Rudy Y, Quan WL. A model study of the effects of the discrete cellular structure on electrical propagation in cardiac tissue. Circ Res. 1987;61:815-823.

600. Gutstein DE, Morley GE, Tamaddon H, Vaidya D, Schneider MD, Chen J, Chien KR, Stuhlmann H, Fishman GI. Conduction slowing and sudden arrhythmic death in mice with cardiacrestricted inactivation of connexin43. Circ Res. 2001;88:333-339

601. Sperelakis N, Mann JE, Jr. Evaluation of electric field changes in the cleft between excitable cells. Journal of theoretical biology. 1977;64:71-96

602. Rohr S, Flueckiger R, Cohen SA. Immunocytochemical localization of na and ca channels in cultured neonatal rat ventricular myocytes. Biophys J. 1999;76:A1-525

603. Mohler PJ, Rivolta I, Napolitano C, LeMaillet G, Lambert S, Priori SG, Bennett V. Nav1.5 e1053k mutation causing brugada syndrome blocks binding to ankyrin-g and expression of nav1.5 on the surface of cardiomyocytes. Proc Natl Acad Sci U S A. 2004;101:17533-17538

604. Tsumoto K, Ashihara T, Haraguchi R, Nakazawa K, Kurachi Y. Roles of subcellular na+ channel distributions in the mechanism of cardiac conduction. Biophys J. 2011;100:554-563

605. Perloff JK, de Leon AC, Jr., O'Doherty D. The cardiomyopathy of progressive muscular dystrophy. Circulation. 1966;33:625-648 
606. Slucka C. The electrocardiogram in duchenne progressive muscular dystrophy. Circulation. 1968;38:933-940

607. Pearson CM. Muscular dystrophy. Review and recent observations. The American journal of medicine. 1963;35:632-645

608. De Mello WC. Cell coupling and impulse propagation in the failing heart. J Cardiovasc Electrophysiol. 1999;10:1409-1420

609. Costa AR, Panda NC, Yong S, Mayorga ME, Pawlowski GP, Fan K, Penn MS, Laurita KR. Optical mapping of cryoinjured rat myocardium grafted with mesenchymal stem cells. Am J Physiol Heart Circ Physiol. 2012;302:H270-277

610. Gray RA, Pertsov AM, Jalife J. Spatial and temporal organization during cardiac fibrillation. Nature. 1998;392:75-78

611. Lou Q, Fedorov VV, Glukhov AV, Moazami N, Fast VG, Efimov IR. Transmural heterogeneity and remodeling of ventricular excitation-contraction coupling in human heart failure. Circulation. 2011;123:1881-1890

612. Efimov IR, Ermentrout B, Huang DT, Salama G. Activation and repolarization patterns are governed by different structural characteristics of ventricular myocardium: Experimental study with voltage-sensitive dyes and numerical simulations. J Cardiovasc Electrophysiol. 1996;7:512-530.

613. Leon LJ, Roberge FA. Directional characteristics of action potential propagation in cardiac muscle. A model study. Circ Res. 1991;69:378-395.

614. Bayly PV, KenKnight BH, Rogers JM, Hillsley RE, Ideker RE, Smith WM. Estimation of conduction velocity vector fields from epicardial mapping data. IEEE transactions on biomedical engineering. 1998;45:563-571

615. Tamaddon HS, Vaidya D, Simon AM, Paul DL, Jalife J, Morley GE. High-resolution optical mapping of the right bundle branch in connexin 40 knockout mice reveals slow conduction in the specialized conduction system. Circ Res. 2000;87:929-936

616. Nygren A, Clark RB, Belke DD, Kondo C, Giles WR, Witkowski FX. Voltage-sensitive dye mapping of activation and conduction in adult mouse hearts. Ann Biomed Eng. 2000;28:958967

617. Spach MS, Dolber PC, Heidlage JF. Influence of the passive anisotropic properties on directional differences in propagation following modification of the sodium conductance in human atrial muscle. A model of reentry based on anisotropic discontinuous propagation. Circ Res. 1988;62:811-832

618. Nestico PF, Morganroth J, Horowitz LN. New antiarrhythmic drugs. Drugs. 1988;35:286-319.

619. Ragsdale DS, McPhee JC, Scheuer T, Catterall WA. Molecular determinants of statedependent block of na+ channels by local anesthetics. Science (New York, N.Y.). 1994;265:1724-1728

620. Patlak J. Molecular kinetics of voltage-dependent na+ channels. Physiol Rev. 1991;71:10471080.

621. Spach MS, Dolber PC, Heidlage JF, Kootsey JM, Johnson EA. Propagating depolarization in anisotropic human and canine cardiac muscle: Apparent directional differences in membrane capacitance. A simplified model for selective directional effects of modifying the sodium conductance on vmax, tau foot, and the propagation safety factor. Circ Res. 1987;60:206-219

622. Gillespie DT. Exact stochastic simulation of coupled chemical reactions. The Journal of Physical Chemistry. 1977;81:2340-2361

623. Delgado C, Steinhaus B, Delmar M, Chialvo DR, Jalife J. Directional differences in excitability and margin of safety for propagation in sheep ventricular epicardial muscle. Circ Res. 1990;67:97-110 
624. Delmar M, Michaels DC, Johnson T, Jalife J. Effects of increasing intercellular resistance on transverse and longitudinal propagation in sheep epicardial muscle. Circ Res. 1987;60:780785

625. Qu Z, Garfinkel A, Chen PS, Weiss JN. Mechanisms of discordant alternans and induction of reentry in simulated cardiac tissue. Circulation. 2000;102:1664-1670

626. de Diego C, Chen F, Xie Y, Pai RK, Slavin L, Parker J, Lamp ST, Qu Z, Weiss JN, Valderrabano M. Anisotropic conduction block and reentry in neonatal rat ventricular myocyte monolayers. Am J Physiol Heart Circ Physiol. 2011;300:H271-278

627. Hodgkin AL. A note on conduction velocity. J Physiol. 1954;125:221-224

628. Kleber AG, Riegger CB, Janse MJ. Electrical uncoupling and increase of extracellular resistance after induction of ischemia in isolated, arterially perfused rabbit papillary muscle. Circ Res. 1987;61:271-279

629. Spach MS, Kootsey JM. Relating the sodium current and conductance to the shape of transmembrane and extracellular potentials by simulation: Effects of propagation boundaries. IEEE transactions on bio-medical engineering. 1985;32:743-755

630. Josephson IR, Sanchez-Chapula J, Brown AM. Early outward current in rat single ventricular cells. Circ Res. 1984;54:157-162.

631. Anderson JL, Jolivette DM, Fredell PA. Summary of efficacy and safety of flecainide for supraventricular arrhythmias. The American journal of cardiology. 1988;62:62d-66d

632. Hohnloser SH, Zabel M. Short- and long-term efficacy and safety of flecainide acetate for supraventricular arrhythmias. The American journal of cardiology. 1992;70:3A-9A; discussion 9A-10A

633. Zamponi GW, French RJ. Dissecting lidocaine action: Diethylamide and phenol mimic separate modes of lidocaine block of sodium channels from heart and skeletal muscle. Biophys J. 1993;65:2335-2347

634. Zamponi GW, Doyle DD, French RJ. Fast lidocaine block of cardiac and skeletal muscle sodium channels: One site with two routes of access. Biophys J. 1993;65:80-90

635. Ikeda N, Singh BN, Davis LD, Hauswirth O. Effects of flecainide on the electrophysiologic properties of isolated canine and rabbit myocardial fibers. J Am Coll Cardiol. 1985;5:303-310

636. Le Grand B, Le Heuzey JY, Perier P, Peronneau P, Lavergne T, Hatem S, Guize L. Cellular electrophysiological effects of flecainide on human atrial fibres. Cardiovasc Res. 1990;24:232-238

637. Wang ZG, Pelletier LC, Talajic M, Nattel S. Effects of flecainide and quinidine on human atrial action potentials. Role of rate-dependence and comparison with guinea pig, rabbit, and dog tissues. Circulation. 1990;82:274-283.

638. Olsson SB, Edvardsson N. Clinical electrophysiologic study of antiarrhythmic properties of flecainide: Acute intraventricular delayed conduction and prolonged repolarization in regular paced and premature beats using intracardiac monophasic action potentials with programmed stimulation. American heart journal. 1981;102:864-871

639. Follmer CH, Cullinan CA, Colatsky TJ. Differential block of cardiac delayed rectifier current by class ic antiarrhythmic drugs: Evidence for open channel block and unblock. Cardiovasc Res. 1992;26:1121-1130.

640. Moe GK, Mendez C. Physiologic basis of premature beats and sustained tachycardias. The New England journal of medicine. 1973;288:250-254

641. Joyner RW. Modulation of repolarization by electrotonic interactions. Japanese heart journal. 1986;27 Suppl 1:167-183

642. Sampson KJ, Henriquez CS. Electrotonic influences on action potential duration dispersion in small hearts: A simulation study. Am J Physiol Heart Circ Physiol. 2005;289:H350-360

643. Irisawa H. Electrophysiology of single cardiac cells. Jpn J Physiol. 1984;34:375-388. 
644. Starmer CF. The cardiac vulnerable period and reentrant arrhythmias: Targets of anti- and proarrhythmic processes. Pacing Clin Electrophysiol. 1997;20:445-454.

645. Wang Y, Rudy Y. Action potential propagation in inhomogeneous cardiac tissue: Safety factor considerations and ionic mechanism. Am J Physiol Heart Circ Physiol. 2000;278:H1019-1029.

646. Szentadrassy N, Banyasz T, Biro T, Szabo G, Toth BI, Magyar J, Lazar J, Varro A, Kovacs L, Nanasi PP. Apico-basal inhomogeneity in distribution of ion channels in canine and human ventricular myocardium. Cardiovasc Res. 2005;65:851-860

647. Otani NF, Li M, Gilmour RF, Jr. What can nonlinear dynamics teach us about the development of ventricular tachycardia/ventricular fibrillation? Heart Rhythm. 2005;2:1261-1263

648. Fox JJ, McHarg JL, Gilmour RF, Jr. Ionic mechanism of electrical alternans. Am J Physiol Heart Circ Physiol. 2002;282:H516-530

649. Sah R, Ramirez RJ, Oudit GY, Gidrewicz D, Trivieri MG, Zobel C, Backx PH. Regulation of cardiac excitation-contraction coupling by action potential repolarization: Role of the transient outward potassium current (i(to)). J Physiol. 2003;546:5-18

650. Gussak I, Chaitman BR, Kopecky SL, Nerbonne JM. Rapid ventricular repolarization in rodents: Electrocardiographic manifestations, molecular mechanisms, and clinical insights. Journal of electrocardiology. 2000;33:159-170

651. Yamaguchi M, Shimizu M, Ino H, Terai H, Uchiyama K, Oe K, Mabuchi T, Konno T, Kaneda T, Mabuchi $\mathrm{H}$. T wave peak-to-end interval and qt dispersion in acquired long qt syndrome: A new index for arrhythmogenicity. Clinical science (London, England : 1979). 2003;105:671676 